Cochrane Database of Systematic Reviews

\title{
High-flow nasal cannulae for respiratory support in adult intensive care patients (Review)
}

Lewis SR, Baker PE, Parker R, Smith AF

Lewis SR, Baker PE, Parker R, Smith AF.

High-flow nasal cannulae for respiratory support in adult intensive care patients.

Cochrane Database of Systematic Reviews 2017, Issue 5. Art. No.: CD010172.

DOI: 10.1002/14651858.CD010172.pub2.

www.cochranelibrary.com 
TABLE OF CONTENTS

HEADER

ABSTRACT

PLAIN LANGUAGE SUMMARY

SUMMARY OF FINDINGS

BACKGROUND

OBJECTIVES

METHODS

RESULTS

Figure 1.

Figure 2.

Figure 3.

Figure 4.

DISCUSSION

AUTHORS' CONCLUSIONS

ACKNOWLEDGEMENTS

REFERENCES

CHARACTERISTICS OF STUDIES

DATA AND ANALYSES

Analysis 1.1. Comparison 1: HFNC versus standard oxygen therapy, Outcome 1: Treatment failure (escalation of respiratory support to NIV, NIPPV or invasive ventilation)

Analysis 1.2. Comparison 1: HFNC versus standard oxygen therapy, Outcome 2: In-hospital mortality

Analysis 1.3. Comparison 1: HFNC versus standard oxygen therapy, Outcome 3: Important adverse events

Analysis 1.4. Comparison 1: HFNC versus standard oxygen therapy, Outcome 4: Length of ICU stay (days)

Analysis 1.5. Comparison 1: HFNC versus standard oxygen therapy, Outcome 5: Short-term respiratory effects: PaO 2/FiO 2 (mmHg)

Analysis 1.6. Comparison 1: HFNC versus standard oxygen therapy, Outcome 6: Comfort

Analysis 1.7. Comparison 1: HFNC versus standard oxygen therapy, Outcome 7: Long-term respiratory effects: PaO 2/FiO 2 ( $\mathrm{mmHg}$ )

Analysis 1.8. Comparison 1: HFNC versus standard oxygen therapy, Outcome 8: Short-term and long-term respiratory effects: $\mathrm{PaO} 2(\mathrm{mmHg})$

Analysis 1.9. Comparison 1: HFNC versus standard oxygen therapy, Outcome 9: Short-term and long-term respiratory effects: SpO $2(\%)$

Analysis 1.10. Comparison 1: HFNC versus standard oxygen therapy, Outcome 10: Short-term respiratory effects: PaCO 2 (mmHg)

Analysis 1.11. Comparison 1: HFNC versus standard oxygen therapy, Outcome 11: Short-term and long-term respiratory rate (breaths/min)

Analysis 1.12. Comparison 1: HFNC versus standard oxygen therapy, Outcome 12: Length of hospital stay (days) ...................

Analysis 1.13. Comparison 1: HFNC versus standard oxygen therapy, Outcome 13: Refusal to continue with treatment ........... Analysis 2.1. Comparison 2: HFNC versus NIPPV or NIV, Outcome 1: Treatment failure (escalation of respiratory support to NIV, NIPPV or invasive ventilation)

Analysis 2.2. Comparison 2: HFNC versus NIPPV or NIV, Outcome 2: In-hospital mortality

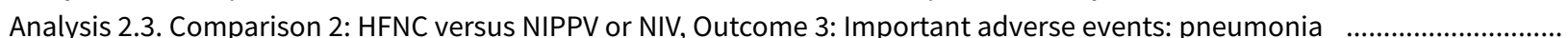

Analysis 2.4. Comparison 2: HFNC versus NIPPV or NIV, Outcome 4: Short-term respiratory effects: PaO 2/FiO 2 (mmHg) .........

Analysis 2.5. Comparison 2: HFNC versus NIPPV or NIV, Outcome 5: Length of ICU stay (days)

Analysis 2.6. Comparison 2: HFNC versus NIPPV or NIV, Outcome 6: Short-term comfort (continuous data)

Analysis 2.7. Comparison 2: HFNC versus NIPPV or NIV, Outcome 7: Duration of respiratory support (hours) ...........................

Analysis 2.8. Comparison 2: HFNC versus NIPPV or NIV, Outcome 8: Long-term respiratory effects: PaO 2/FiO 2 (mmHg) .........

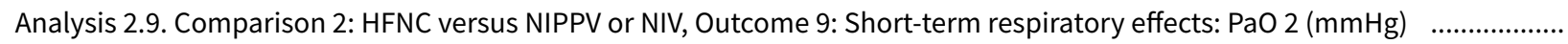

Analysis 2.10. Comparison 2: HFNC versus NIPPV or NIV, Outcome 10: Short-term and long-term respiratory effects: PaCO 2 (mmHg)

Analysis 2.11. Comparison 2: HFNC versus NIPPV or NIV, Outcome 11: Short-term respiratory effects: breaths/min

Analysis 2.12. Comparison 2: HFNC versus NIPPV or NIV, Outcome 12: Dyspnoea (any improvement) 
[Intervention Review]

\section{High-flow nasal cannulae for respiratory support in adult intensive care patients}

Sharon R Lewis ${ }^{1}$, Philip E Baker², Roses Parker ${ }^{3}$, Andrew F Smith ${ }^{4}$

1Lancaster Patient Safety Research Unit, Royal Lancaster Infirmary, Lancaster, UK. 2Academic Centre, Oxford University Hospitals NHS Trust, Oxford, UK. ${ }^{3}$ Cochrane MOSS Network, c/o Cochrane Pain Palliative and Supportive Care Group, Oxford University Hospitals NHS Foundation Trust, Oxford, UK. ${ }^{4}$ Department of Anaesthesia, Royal Lancaster Infirmary, Lancaster, UK

Contact address: Sharon R Lewis, Sharon.Lewis@mbht.nhs.uk.

Editorial group: Cochrane Emergency and Critical Care Group.

Publication status and date: Edited (no change to conclusions), published in Issue 2, 2021.

Citation: Lewis SR, Baker PE, Parker R, Smith AF. High-flow nasal cannulae for respiratory support in adult intensive care patients. Cochrane Database of Systematic Reviews 2017, Issue 5. Art. No.: CD010172. DOI: 10.1002/14651858.CD010172.pub2.

Copyright ( 2017 The Cochrane Collaboration. Published by John Wiley \& Sons, Ltd.

\section{A B S T R A C T}

\section{Background}

High-flow nasal cannulae (HFNC) deliver high flows of blended humidified air and oxygen via wide-bore nasal cannulae and may be useful in providing respiratory support for adults experiencing acute respiratory failure, or at risk of acute respiratory failure, in the intensive care unit (ICU). This is an update of an earlier version of the review.

\section{Objectives}

To assess the effectiveness of HFNC compared to standard oxygen therapy, or non-invasive ventilation (NIV) or non-invasive positive pressure ventilation (NIPPV), for respiratory support in adults in the ICU.

\section{Search methods}

We searched CENTRAL, MEDLINE, Embase, CINAHL, Web of Science, and the Cochrane COVID-19 Register (17 April 2020), clinical trial registers (6 April 2020) and conducted forward and backward citation searches.

\section{Selection criteria}

We included randomized controlled studies (RCTs) with a parallel-group or cross-over design comparing HFNC use versus other types of non-invasive respiratory support (standard oxygen therapy via nasal cannulae or mask; or NIV or NIPPV which included continuous positive airway pressure and bilevel positive airway pressure) in adults admitted to the ICU.

\section{Data collection and analysis}

We used standard methodological procedures as expected by Cochrane.

\section{Main results}

We included 31 studies (22 parallel-group and nine cross-over designs) with 5136 participants; this update included 20 new studies. Twenty-one studies compared HFNC with standard oxygen therapy, and 13 compared HFNC with NIV or NIPPV; three studies included both comparisons. We found 51 ongoing studies (estimated 12,807 participants), and 19 studies awaiting classification for which we could not ascertain study eligibility information.

In 18 studies, treatment was initiated after extubation. In the remaining studies, participants were not previously mechanically ventilated.

\section{HFNC versus standard oxygen therapy}


HFNC may lead to less treatment failure as indicated by escalation to alternative types of oxygen therapy (risk ratio (RR) $0.62,95 \%$ confidence interval ( $\mathrm{Cl}) 0.45$ to $0.86 ; 15$ studies, 3044 participants; low-certainty evidence). HFNC probably makes little or no difference in mortality when compared with standard oxygen therapy (RR 0.96, 95\% CI 0.82 to $1.11 ; 11$ studies, 2673 participants; moderate-certainty evidence). HFNC probably results in little or no difference to cases of pneumonia (RR $0.72,95 \% \mathrm{Cl} 0.48$ to $1.09 ; 4$ studies, 1057 participants; moderate-certainty evidence), and we were uncertain of its effect on nasal mucosa or skin trauma (RR 3.66, $95 \% \mathrm{Cl} 0.43$ to $31.48 ; 2$ studies, 617 participants; very low-certainty evidence). We found low-certainty evidence that HFNC may make little or no difference to the length of ICU stay according to the type of respiratory support used (MD 0.12 days, $95 \% \mathrm{Cl}-0.03$ to $0.27 ; 7$ studies, 1014 participants). We are uncertain whether HFNC made any difference to the ratio of partial pressure of arterial oxygen to the fraction of inspired oxygen $\left(\mathrm{PaO}_{2} /\right.$ $\mathrm{FiO}_{2}$ ) within 24 hours of treatment (MD $10.34 \mathrm{mmHg}, 95 \% \mathrm{Cl}-17.31$ to 38; 5 studies, 600 participants; very low-certainty evidence). We are uncertain whether HFNC made any difference to short-term comfort (MD $0.31,95 \% \mathrm{Cl}-0.60$ to 1.22; 4 studies, 662 participants, very lowcertainty evidence), or to long-term comfort (MD 0.59, 95\% Cl -2.29 to 3.47; 2 studies, 445 participants, very low-certainty evidence).

\section{HFNC versus NIV or NIPPV}

We found no evidence of a difference between groups in treatment failure when HFNC were used post-extubation or without prior use of mechanical ventilation (RR $0.98,95 \% \mathrm{Cl} 0.78$ to 1.22 ; 5 studies, 1758 participants; low-certainty evidence), or in-hospital mortality (RR $0.92,95 \% \mathrm{Cl} 0.64$ to 1.31 ; 5 studies, 1758 participants; low-certainty evidence). We are very uncertain about the effect of using HFNC on incidence of pneumonia (RR $0.51,95 \% \mathrm{Cl} 0.17$ to $1.52 ; 3$ studies, 1750 participants; very low-certainty evidence), and HFNC may result in little or no difference to barotrauma (RR $1.15,95 \%$ Cl 0.42 to 3.14 ; 1 study, 830 participants; low-certainty evidence). HFNC may make little or no difference to the length of ICU stay (MD -0.72 days, $95 \% \mathrm{CI}-2.85$ to 1.42; 2 studies, 246 participants; low-certainty evidence). The ratio of $\mathrm{PaO}_{2} / \mathrm{FiO}_{2}$ may be lower up to 24 hours with $\mathrm{HFNC}$ use (MD $-58.10 \mathrm{mmHg}, 95 \% \mathrm{Cl}-71.68$ to $-44.51 ; 3$ studies, 1086 participants; lowcertainty evidence). We are uncertain whether HFNC improved short-term comfort when measured using comfort scores (MD $1.33,95 \% \mathrm{Cl}$ 0.74 to $1.92 ; 2$ studies, 258 participants) and responses to questionnaires (RR 1.30, 95\% $\mathrm{Cl} 1.10$ to $1.53 ; 1$ study, 168 participants); evidence for short-term comfort was very low certainty. No studies reported on nasal mucosa or skin trauma.

\section{Authors' conclusions}

HFNC may lead to less treatment failure when compared to standard oxygen therapy, but probably makes little or no difference to treatment failure when compared to NIV or NIPPV. For most other review outcomes, we found no evidence of a difference in effect. However, the evidence was often of low or very low certainty. We found a large number of ongoing studies; including these in future updates could increase the certainty or may alter the direction of these effects.

\section{PLAIN LANGUAGE SUMMARY}

\section{High-flow nasal cannulae for breathing support in adult intensive care patients}

\section{Review question}

Are high-flow nasal cannulae (HFNC) a helpful treatment option for adult patients in the intensive care unit (ICU) who need breathing support?

\section{Background}

People in the ICU may need support to breathe and HFNC are one option for this. HFNC deliver warm air and oxygen through small plastic tubes that sit inside the nostrils. The airflow is at a higher rate each minute than standard oxygen therapy (which is not always warmed and may be delivered through a plastic face mask or nasal cannulae). Other support options include non-invasive ventilation (NIV) or non-invasive positive pressure ventilation (NIPPV). These approaches use mild pressure to push air into the lungs through tightly-fitting face masks or a helmet covering the entire head. Invasive mechanical ventilation provides the highest level of support, using a ventilator (artificial breathing machine) to push air in and out of the lungs through a plastic tube inserted into the windpipe.

\section{Search date}

The evidence is current to April 2020.

\section{Study characteristics}

All participants were adults (16 years or older) requiring support to breathe in an ICU. Most participants had respiratory failure (in which the lungs are unable to get enough oxygen into the blood) or had just been taken off a ventilator and needed support to transition to independent breathing.

We searched for randomized controlled trials; these trials give participants an equal chance to be in either trial group and provide the best evidence. We included trials that compared HFNC with standard oxygen therapy or NIV or NIPPV. We included 31 studies with 5136 participants, 51 ongoing studies and 19 studies awaiting classification. Fourteen studies were funded by manufacturers of breathing equipment. 


\section{Key results}

HFNC compared to standard oxygen therapy

We found that using HFNC may reduce the need for patients to change to another type of breathing support (treatment failure). We found no evidence of a difference between the two interventions for: hospital deaths, length of ICU stay, pneumonia (lung infection), skin damage caused by tubes or masks in contact with the face, comfort while patients received breathing support, or in how well either treatment provided oxygen to the blood.

\section{HFNC compared to NIV or NIPPV}

We found no evidence of a difference in treatment failure between using HFNC and NIV or NIPPV. We also found no evidence of a difference for hospital deaths, length of ICU stay, pneumonia, or barotrauma (damage to the body caused by differences in pressure inside and outside the body). NIV or NIPPV may improve how well oxygen gets into the blood. We are uncertain whether HFNC could be more comfortable for patients in the first 24 hours of use. No studies reported skin damage.

\section{Quality of evidence}

We used a rating scale to decide the quality of the evidence in these trials. When we rate evidence as very low-certainty, it means that we are very uncertain about the reliability of the results. High-certainty means that we are very confident about the results.

We did not always have evidence from enough studies to give us confidence in the key results. Sometimes our findings changed if we removed studies that were less well reported (e.g. regarding how participants were allocated to a treatment). We also found some variation between study results for some outcomes. We are moderately certain in our findings that HFNC did not influence hospital deaths and pneumonia when compared to standard oxygen therapy, but for all other outcomes, we judged the evidence to be of low or very low certainty. This means that our confidence in these results is limited or very limited, and the real effect may be very different.

\section{Conclusion}

HFNC may lead to less treatment failure when compared to standard oxygen therapy, but probably makes little or no difference when compared to NIV or NIPPV. For most other review outcomes, we found no reliable evidence of a difference in effect. However, we identified another 51 ongoing trials and we plan to include these in future updates of the review. When these trials are incorporated, we may reach different conclusions about whether HFNC is helpful for breathing support in adult ICU patients. 
SUMMARY OF FINDINGS

Summary of findings 1. HFNC compared to standard oxygen therapy for respiratory support in adult intensive care patients

High-flow nasal cannulae compared to standard oxygen therapy for respiratory support in adult intensive care patients

Population: adults in the ICU, requiring respiratory support

Setting: ICUs. In this review, these ICUs were in: Australia; Belgium; China; France; Italy; New Zealand; Spain; Taiwan; Thailand; UK.

Intervention: oxygen delivered via HFNC, initiated after extubation from invasive mechanical ventilation or without prior use of invasive mechanical ventilation

Comparison: standard oxygen therapy delivered via nasal cannula or face mask

\begin{tabular}{|c|c|c|c|c|c|c|}
\hline \multirow[t]{2}{*}{ Outcomes } & \multicolumn{2}{|c|}{$\begin{array}{l}\text { Anticipated absolute effects }{ }^{*}(95 \% \\
\mathrm{CI})\end{array}$} & \multirow[t]{2}{*}{$\begin{array}{l}\text { Relative effect } \\
(95 \% \mathrm{CI})\end{array}$} & \multirow{2}{*}{$\begin{array}{l}\text { Number of par- } \\
\text { ticipants } \\
\text { (studies) }\end{array}$} & \multirow{2}{*}{$\begin{array}{l}\text { Certainty of } \\
\text { the evidence } \\
\text { (GRADE) }\end{array}$} & \multirow[t]{2}{*}{ Comments } \\
\hline & $\begin{array}{l}\text { Risk with stan } \\
\text { dard oxygen } \\
\text { therapy }\end{array}$ & Risk with HFNC & & & & \\
\hline \multirow{2}{*}{$\begin{array}{l}\text { Treatment failure (escalation of } \\
\text { respiratory therapy to NIV, NIPPV } \\
\text { or invasive ventilation) }\end{array}$} & \multicolumn{2}{|c|}{ Study population } & \multirow{2}{*}{$\begin{array}{l}\text { RR } 0.62 \\
(0.45 \text { to } 0.86)\end{array}$} & \multirow{2}{*}{$\begin{array}{l}3044 \\
\text { (15 studies) }\end{array}$} & \multirow{2}{*}{$\begin{array}{l}\oplus \oplus \ominus \ominus \\
\text { Low } a\end{array}$} & \\
\hline & 261 per 1000 & $\begin{array}{l}162 \text { per } 1000 \\
\text { (117 to } 224)\end{array}$ & & & & \\
\hline \multirow{2}{*}{$\begin{array}{l}\text { In-hospital mortality } \\
\text { (up to } 90 \text { days; included studies } \\
\text { reported in-hospital mortality, } \\
\text { and mortality up to } 28 \text { days, up to } \\
\text { ICU discharge, and at unspecified } \\
\text { time points) }\end{array}$} & \multicolumn{2}{|c|}{ Study population } & \multirow{2}{*}{$\begin{array}{l}\text { RR } 0.96 \\
\text { (0.82 to } 1.11)\end{array}$} & \multirow{2}{*}{$\begin{array}{l}2673 \\
\text { (11 studies) }\end{array}$} & \multirow{2}{*}{$\begin{array}{l}\oplus \oplus \oplus \ominus \\
\text { Moderate b }\end{array}$} & \multirow[t]{2}{*}{-} \\
\hline & 163 per 1000 & $\begin{array}{l}156 \text { per } 1000 \\
\text { (134 to } 181)\end{array}$ & & & & \\
\hline \multirow{2}{*}{$\begin{array}{l}\text { Adverse events } \\
\text { Respiratory infection (pneumo- } \\
\text { nia) }\end{array}$} & \multicolumn{2}{|c|}{ Study population for pneumonia } & \multirow{2}{*}{$\begin{array}{l}\text { RR } 0.72 \\
\text { (0.48 to } 1.09 \text { ) }\end{array}$} & \multirow{2}{*}{$\begin{array}{l}1057 \\
\text { (4 studies) }\end{array}$} & $\oplus \oplus \oplus \ominus$ & - \\
\hline & 84 per 1000 & $\begin{array}{l}61 \text { per } 1000 \\
(40 \text { to } 92)\end{array}$ & & & Moderate $\mathrm{c}$ & - \\
\hline \multirow{2}{*}{ Nasal mucosa or skin trauma } & \multicolumn{2}{|c|}{$\begin{array}{l}\text { Study population for nasal mucosa or } \\
\text { skin trauma }\end{array}$} & \multirow[t]{2}{*}{$\begin{array}{l}\text { RR } 3.66 \\
\text { (0.43 to } 31.48 \text { ) }\end{array}$} & \multirow[t]{2}{*}{$\begin{array}{l}617 \\
\text { ( } 2 \text { studies) }\end{array}$} & \multirow{2}{*}{$\begin{array}{l}\oplus \ominus \ominus \ominus \\
\text { Very low } d\end{array}$} & \\
\hline & 3 per 1000 & $\begin{array}{l}12 \text { per } 1000 \\
(1 \text { to } 103)\end{array}$ & & & & \\
\hline Length of ICU stay & 1.88 days & $\begin{array}{l}\text { MD } 0.12 \text { days high- } \\
\text { er }\end{array}$ & - & $\begin{array}{l}1014 \\
\text { (7 studies) }\end{array}$ & $\oplus \oplus \ominus \ominus$ & $\begin{array}{l}\text { In addition, } \\
\text { dian length }\end{array}$ \\
\hline
\end{tabular}




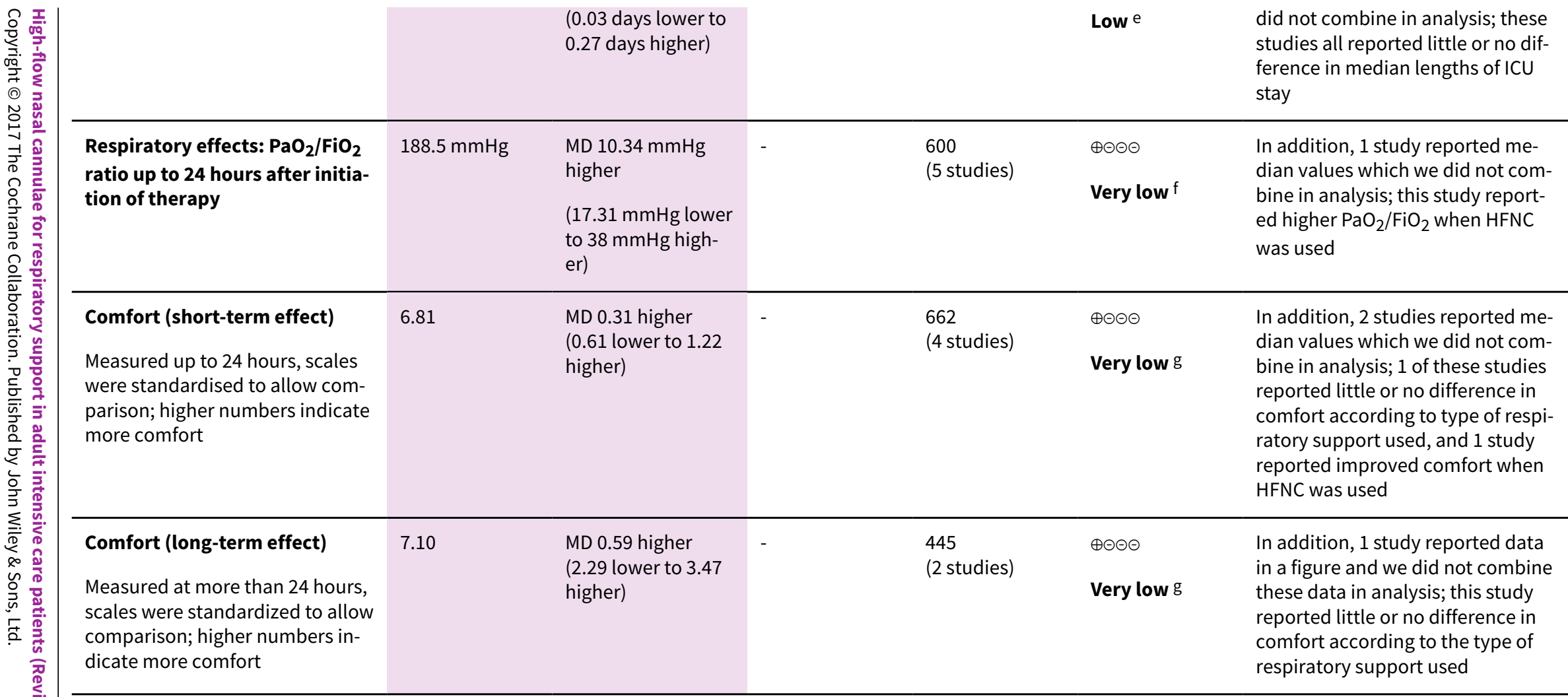

${ }^{*}$ The risk in the intervention group (and its $95 \%$ confidence interval) is based on the assumed risk in the comparison group and the relative effect of the intervention (and its $95 \% \mathrm{Cl}$ ). For length of stay, $\mathrm{PaO}_{2} / \mathrm{FiO}_{2}$ and comfort, we present baseline risk values for standard oxygen therapy as the weighted mean values reported in included studies for each outcome. For comfort, these values are scores on a scale from 0 (least comfort) to 10 (most comfort).

Cl: confidence interval; HFNC: high-flow nasal cannulae; ICU: intensive care unit; MD: mean difference; $\mathbf{P a O}_{2} / \mathbf{F i O}_{2}$ : ratio of partial pressure of arterial oxygen to the fraction of inspired oxygen; RR: risk ratio; SMD: standardized mean difference

\section{GRADE Working Group grades of evidence}

High certainty: we are very confident that the true effect lies close to that of the estimate of the effect

Moderate certainty: we are moderately confident in the effect estimate: The true effect is likely to be close to the estimate of the effect, but there is a possibility that it is substantially different

Low certainty: our confidence in the effect estimate is limited: The true effect may be substantially different from the estimate of the effect

Very low certainty: we have very little confidence in the effect estimate: The true effect is likely to be substantially different from the estimate of effect

aWe downgraded by one level for inconsistency because we noted a moderate level of statistical heterogeneity; we also noted more variation in the data from studies in which respiratory support was given post-extubation which we could not explain. We also downgraded by one level for study limitations because we judged some studies to have an 
bWe downgraded by one level for inconsistency because we noted inconsistencies in the data which we expected were caused by the differences in illness severity of participants in the studies which is likely to impact on mortality rates between studies.

cWe downgraded by one level for imprecision because only four studies contributed evidence for this outcome.

dWe downgraded by three levels: we downgraded two levels for imprecision because only two studies contributed evidence, of which only one reported events. We also downgraded by one level for study limitations because this study did not report study trials registration and we could not be certain whether it was at risk of selective reporting bias. eWe downgraded by two levels: we downgraded by one level for inconsistency because we noted variation in the lengths of stay between studies which we expected was because of different illness severity between study participants. We also downgraded by one level for study limitations because we noted some high risks of bias in some included studies. fWe downgraded by three levels: we downgraded by two levels for inconsistency because we noted a substantial amount of statistical heterogeneity which we could not explain, and we downgraded by one level for study limitations because we noted differences in the effect estimate when we excluded studies at unclear or high risks of selection bias in sensitivity analyses.

gWe downgraded by three levels: we downgraded by two levels for inconsistency because we noted a substantial level of statistical heterogeneity and variation in the data between the studies which we could not explain. We also downgraded by one level for imprecision because few studies contributed data for this outcome.

\section{Summary of findings 2. HFNC compared to NIPPV or NIV for respiratory support in adult intensive care patients}

\section{High-flow nasal cannulae compared to NIPPV or NIV for respiratory support in adult intensive care patients}

Population: adults in the ICU, requiring respiratory support

Setting: ICUs. In this review, these ICUs were in: Belgium, China, France, Saudi Arabia, and Spain

Intervention: oxygen delivered via HFNC, initiated after extubation from invasive mechanical ventilation or without prior use of invasive mechanical ventilation Comparison: oxygen delivered via NIV or NIPPV (using BiPAP)

\begin{tabular}{|c|c|c|c|c|c|c|}
\hline \multirow[t]{2}{*}{ Outcomes } & \multicolumn{2}{|c|}{$\begin{array}{l}\text { Anticipated absolute effects }{ }^{*}(95 \% \\
\mathrm{CI})\end{array}$} & \multirow[t]{2}{*}{$\begin{array}{l}\text { Relative effect } \\
(95 \% \mathrm{Cl})\end{array}$} & \multirow{2}{*}{$\begin{array}{l}\text { Number of par- } \\
\text { ticipants } \\
\text { (studies) }\end{array}$} & \multirow{2}{*}{$\begin{array}{l}\text { Certainty of } \\
\text { the evidence } \\
\text { (GRADE) }\end{array}$} & \multirow[t]{2}{*}{ Comments } \\
\hline & $\begin{array}{l}\text { Risk with NIP. } \\
\text { PV or NIV }\end{array}$ & Risk with HFNC & & & & \\
\hline \multirow{2}{*}{$\begin{array}{l}\text { Treatment failure (esca- } \\
\text { lation of respiratory thera- } \\
\text { py to NIV, NIPPV or invasive } \\
\text { ventilation) }\end{array}$} & \multicolumn{2}{|c|}{ Study population } & \multirow{2}{*}{$\begin{array}{l}\text { RR } 0.98 \\
\text { (0.78 to } 1.22 \text { ) }\end{array}$} & \multirow{2}{*}{$\begin{array}{l}1758 \\
\text { (5 studies) }\end{array}$} & \multirow{2}{*}{$\begin{array}{l}\oplus \oplus \ominus \ominus \\
\text { Low } a\end{array}$} & \multirow{2}{*}{$\begin{array}{l}\text { We conducted subgroup analysis and } \\
\text { found no evidence of a difference in treat- } \\
\text { ment failure when used post-extubation } \\
\text { (RR } 1.12,95 \% \mathrm{Cl} 0.89 \text { to } 1.41 ; 3 \text { studies, } \\
1472 \text { participants) and without prior use } \\
\text { of mechanical ventilation (RR } 0.77,95 \% \mathrm{Cl} \\
0.58 \text { to } 1.03 ; 2 \text { studies, } 286 \text { participants) }\end{array}$} \\
\hline & 202 per 1000 & $\begin{array}{l}198 \text { per } 1000 \\
\text { (158 to } 247)\end{array}$ & & & & \\
\hline \multirow{2}{*}{$\begin{array}{l}\text { In-hospital mortality } \\
\text { (up to } 90 \text { days; included } \\
\text { studies reported in-hospital } \\
\text { mortality, and mortality up } \\
\text { to } 28 \text { days and up to ICU dis- } \\
\text { charge) }\end{array}$} & \multicolumn{2}{|c|}{ Study population } & \multirow{2}{*}{$\begin{array}{l}\text { RR } 0.92 \\
(0.64 \text { to } 1.31)\end{array}$} & \multirow{2}{*}{$\begin{array}{l}1758 \\
\text { (5 studies) }\end{array}$} & $\oplus \oplus \ominus \ominus$ & \multirow[t]{2}{*}{-} \\
\hline & 136 per 1000 & $\begin{array}{l}126 \text { per } 1000 \\
(87 \text { to } 179)\end{array}$ & & & $\operatorname{Low}^{a}$ & \\
\hline
\end{tabular}




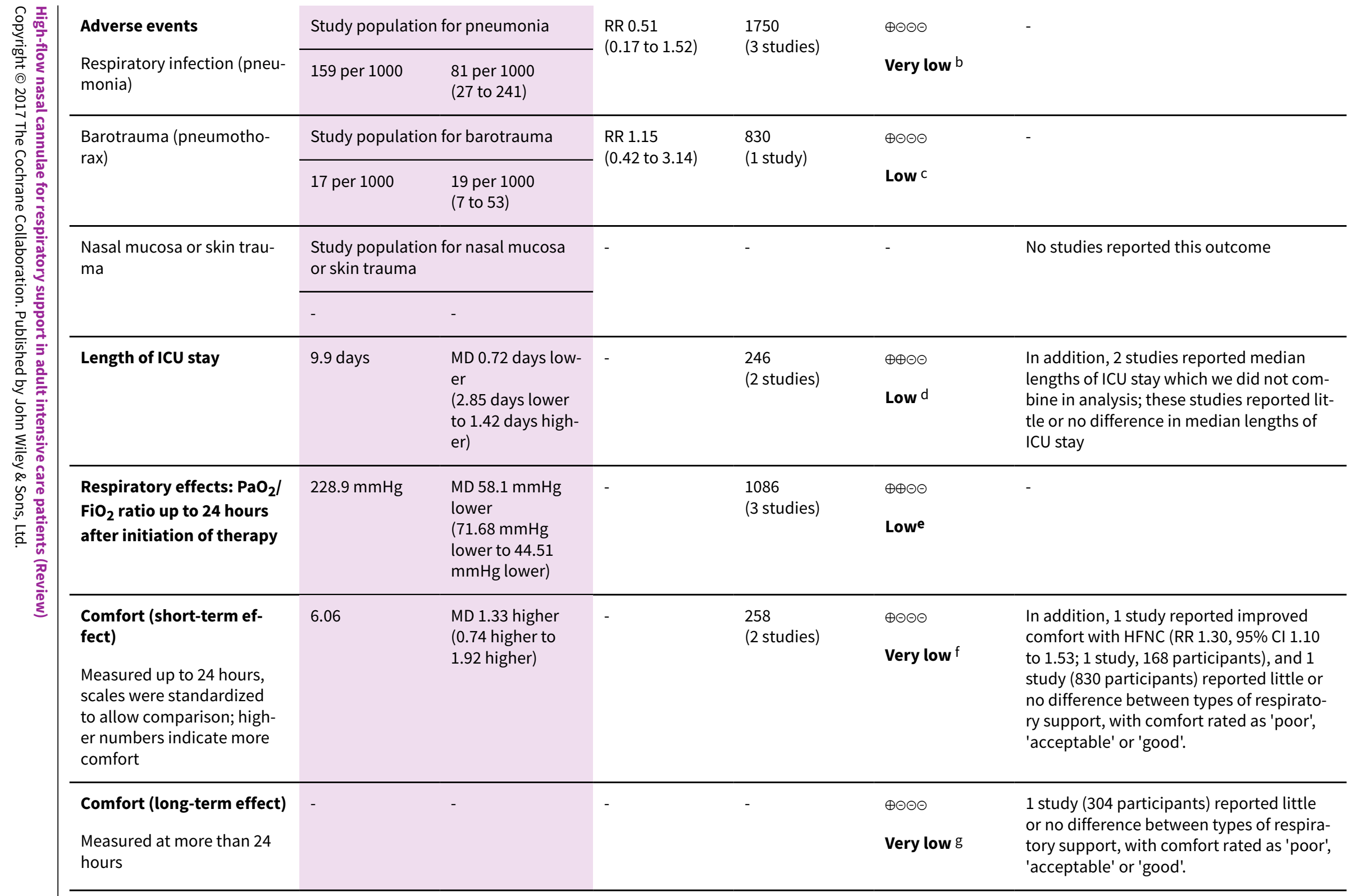

${ }^{*}$ The risk in the intervention group (and its $95 \%$ confidence interval) is based on the assumed risk in the comparison group and the relative effect of the intervention (and its $95 \% \mathrm{CI}$ ). We present baseline risk values for NIPPV/NIV as the weighted mean values reported in included studies for each outcome. For comfort, these values are a score from 0 (least comfort) to 10 (most comfort). 
CI: Confidence interval; HFNC: high-flow nasal cannulae; ICU: intensive care unit; MD: mean difference; NIPPV: non-invasive positive pressure ventilation; NIV: non-invasive ventilation; $\mathbf{P a O}_{2} / \mathrm{FiO}_{2}$ : ratio of partial pressure of arterial oxygen to the fraction of inspired oxygen; RR: risk ratio; SMD: standardized mean difference

\section{GRADE Working Group grades of evidence}

High certainty: We are very confident that the true effect lies close to that of the estimate of the effect

Moderate certainty: We are moderately confident in the effect estimate: The true effect is likely to be close to the estimate of the effect, but there is a possibility that it is substantially different

Low certainty: Our confidence in the effect estimate is limited: The true effect may be substantially different from the estimate of the effect

Very low certainty: We have very little confidence in the effect estimate: The true effect is likely to be substantially different from the estimate of effect

aWe downgraded by two levels: we downgraded by one level for inconsistency because we noted some variation in the results which we could not explain. We also downgraded by one level for study limitations because we judged one study to have a high risk of bias owing to the use of alternative treatment between intermittent HFNC use.

bWe downgraded by three levels: we downgraded by two levels for inconsistency because we noted variation in the results of individual studies and a substantial level of statistical

heterogeneity, and by one level for study limitations because we judged one study to have a high risk of bias owing to the use of alternative treatment between intermittent HFNC use.

cWe downgraded by two levels for imprecision because only one study contributed evidence to this outcome and we noted a wide $\mathrm{Cl}$ in the effect.

dWe downgraded by two levels: we downgraded by one level for inconsistency because we noted a wide variation in length of stay within studies, and by one level for study limitations because we judged one study to have a high risk of bias owing to the use of alternative treatment between intermittent HFNC use.

eWe downgraded by two levels: we downgraded by one level for inconsistency because one study had a particularly wide $\mathrm{Cl}$ and we noted differences in $\mathrm{PaO}_{2} / \mathrm{FiO}{ }_{2}$ between studies which could be explained by the different reasons for needing respiratory support between studies. We also downgraded by one level for study limitations because we judged one study to have a high risk of bias owing to the use of alternative treatment between intermittent HFNC use.

fWe downgraded by three levels: we downgraded by two levels for inconsistency because we noted some variation between study results, and by one level for study limitations because we judged one study to have a high risk of bias owing to the use of alternative treatment between intermittent HFNC use.

gWe downgraded by three levels: we downgraded by two levels for imprecision because only one study contributed evidence for this outcome, and one level for study limitations because we noted a high rate of attrition for comfort scores measured at day 3 . 


\section{B A C K G R O U N D}

\section{Description of the condition}

Acute respiratory failure and the subsequent need for respiratory support is a frequent cause of admission of adults to an intensive care unit (ICU) (Behrendt 2000). There are multiple pathological processes which lead to acute respiratory failure, making it difficult to summarise all the possible conditions which would result in a person requiring respiratory support in an ICU environment. In broad terms, respiratory failure in critically unwell people can be considered due to hypoxaemia, ventilatory failure or both (Shelly 1999). These can be a result of conditions such as community or hospital-acquired pneumonia, sepsis, aspiration or drowning, pneumonia related to being immunocompromised, acute exacerbations of chronic obstructive pulmonary disease (COPD), after surgery (particularly cardiothoracic surgery) and many others. Although drugs may improve some types of respiratory failure (Lewis 2019), respiratory support is the mainstay of treatment. This respiratory support can be provided to the patient in an invasive or non-invasive manner.

\section{Description of the intervention}

Invasive mechanical ventilation involves the insertion of an artificial airway (an endotracheal or tracheostomy tube). Although this is regarded as a life-saving treatment, it comes with multiple inherent risks to patients. These risks include the development of ventilator-induced lung injury (Gattinoni 2012), ventilatorassociated pneumonia (Muscadere 2008), neurocognitive sequelae associated with prolonged sedation (Morandi 2011; Nelson 2000), and increased length of ICU and hospital stay (Safdar 2005). Therefore, when possible, invasive mechanical ventilation should be avoided. However, intubation and mechanical ventilation are inevitable if the patient has stopped breathing or is unable to maintain their airway (Nava 2009).

Non-invasive respiratory support, when possible, is the preferred method of respiratory support and can be delivered via any of the following approaches (O'Driscoll 2008).

- Low-flow nasal cannulae (LFNC).

- Simple face mask.

- Venturi mask.

- Non-rebreather mask.

- Non-invasive ventilation or non-invasive positive-pressure ventilation (NIPPV).

- High-flow nasal cannulae (HFNC).

The type of delivery device chosen depends largely on the severity and the cause of the patient's acute respiratory failure, and each device provides benefits and drawbacks that determine its usefulness in clinical practice.

Physicians use LFNC for patients requiring minimal respiratory support in the form of supplemental oxygen to maintain adequate oxygenation. These cannulae deliver dry oxygen at 1 to 6 litres per minute via small prongs approximately $1.5 \mathrm{~cm}$ long, which sit just inside the nostrils (O'Driscoll 2008). Although they are generally well tolerated by patients (Zevola 2001), delivery of higher flows of oxygen through LFNC is not practicable owing to the drying and irritating effects of cold dry gas on the mucosa (Costello 1995; Cuquemelle 2012; Lellouche 2002).
Delivery of oxygen via a face mask is necessary if the patient has higher oxygen requirements than can be achieved with LFNC. Simple face masks can deliver 5 to 10 litres per minute of oxygen. For patients requiring increased oxygen and higher flows to maintain adequate oxygenation, non-rebreather masks can deliver 10 to 15 litres per minute of oxygen (O'Driscoll 2008). Oxygen may be supplemented with humidification by some devices. Simple face masks and non-rebreather masks are capable of delivering relatively high oxygen concentrations; therefore, they are generally unsuitable for patients with chronic obstructive pulmonary disease (COPD), who may retain carbon dioxide. For hypercapnoeic patients with COPD, oxygen concentration can be regulated by a Venturi mask, which can deliver between $24 \%$ and $60 \%$ oxygen at a flow of 2 to 15 litres per minute (O'Driscoll 2008). Although face masks are effective for delivering oxygen to patients with mild to moderate acute respiratory failure, they can be poorly tolerated when compared with nasal cannulae owing to discomfort and feelings of claustrophobia. This may lead to reduced compliance as a result of frequent removal and subsequent treatment interruption (Sasaki 2003).

HFNC, which have been used in the neonatal setting for some years (Wilkinson 2016), are a relatively new method of delivering respiratory support to adults experiencing acute respiratory failure. Cannulae are approximately $1.5 \mathrm{~cm}$ long and $0.5 \mathrm{~cm}$ in diameter and, as with LFNC, sit just inside the nostrils. A gas flow of up to 60 litres per minute can be delivered because the gas is warmed and humidified, making it less irritating to the nasal mucosa (Papazian 2016). For this review, HFNC will be defined as humidified oxygen delivered via nasal cannulae at a rate greater than 20 litres per minute. Very few adverse reactions have been reported with HFNC use and those reported consist of minor complaints of a runny nose (Price 2008) and some discomfort with heat or flow rate (Roca 2010).

NIPPV can be used in patients who not only require supplemental oxygen but also need support for the mechanical process of ventilation (Mehta 2001). A blend of oxygen and air is delivered at a prescribed fraction of inspired oxygen $\left(\mathrm{FiO}_{2}\right)$ via a tightfitting mask (nasal mask, oronasal mask, or full face mask). Additionally, continuous positive airway pressure (CPAP) or bilevel positive airway pressure ventilation (BiPAP) is delivered to improve alveolar recruitment, improve gas exchange, and decrease the work of breathing (Mehta 2001). Although CPAP is not a true ventilatory mode, it is often referred to as NIPPV in clinical practice (Nava 2009). Substantial available data show that NIPPV improves outcomes among patients requiring respiratory support owing to cardiogenic pulmonary oedema or acute exacerbations of COPD, and also among patients weaning from invasive mechanical ventilation (Nava 2009). However, its relevance for patients with hypoxaemic acute respiratory failure is less clearly defined (Nava 2009). Despite showing clear benefit for certain conditions, NIPPV inhibits mobilization, is associated with gastric distension, restricts effective communication and oral nutrition, and is poorly tolerated by some patients owing to discomfort (Bello 2016; Gregoretti 2002; Mehta 2001).

Although the conventional non-invasive delivery devices listed above provide important therapies in the range of respiratory support available to treat patients with acute respiratory failure, it is evident that they have limitations that can impact their usefulness in clinical practice. Failure of these devices to provide adequate respiratory support and to correct acute respiratory 
failure often results in the need for intubation and mechanical ventilation.

\section{How the intervention might work}

HFNC can deliver blended humidified air and oxygen via wide-bore nasal cannulae at a prescribed $\mathrm{FiO}_{2}$ at high-flow rates. HFNC do not need to be removed during oral hygiene care or when patients talk, eat, or drink, resulting in less frequent interruptions to therapy. In the growing body of evidence gathered when effects of HFNC are investigated, improvements in oxygenation (Corley 2011; Parke 2009; Roca 2010; Sztrymf 2011; Sztrymf 2011a), respiratory rate (Corley 2011; Roca 2010; Sztrymf 2011; Sztrymf 2011a), dyspnoea (Corley 2011; Roca 2010; Sztrymf 2011), and patient comfort (Corley 2011; Roca 2010) have been reported in recent observational studies.

\section{Suggested mechanisms of action of HFNC consist of:}

- flushing of anatomical dead space due to high gas flow, functionally reducing dead space and improving respiratory efficiency (Dysart 2009);

- generation of positive airway pressure (Corley 2011; Groves 2007; Parke 2009), which increases functional residual capacity and improves alveolar recruitment;

- improved ability to meet high inspiratory flow demands among patients requiring respiratory support and to deliver a more accurate $\mathrm{FiO}_{2}$ through less dilution by entrainment of room air (Dysart 2009); and

- ability to deliver optimal humidification, leading to enhanced mucociliary transport (Salah 1988) and improved patient comfort (Chanques 2009).

We conducted this review to compare the efficacy and safety of HFNC versus other methods of non-invasive respiratory support in adult patients admitted to the ICU.

\section{Why it is important to do this review}

It has been demonstrated that HFNC offer some immediate physiological benefit for patients requiring respiratory support, but it remains to be determined whether they offer any clinically important benefit and improve patient outcomes, such as by preventing progression to invasive mechanical ventilation and reducing mortality. Despite increased popularity as a treatment modality for respiratory support, there is still uncertainty about which patient populations benefit most from HFNC as compared to other therapies (Curley 2015; Demoule 2015; Levy 2016; Nishimura 2015). Individual studies may tend to focus on surrogate outcomes or may be underpowered to detect effects on clinically important outcomes. This is an update of a previously published Cochrane Review (Corley 2017). In the previous version of this review, we found 11 eligible studies but this was insufficient to demonstrate with any certainty whether HFNC is a more effective or safe oxygen delivery device compared with other oxygenation devices in adults in the ICU. Given the COVID-19 pandemic, in which people are admitted to the ICU requiring respiratory support caused by SARS$\mathrm{CoV}-2$, we believe there is an urgent need to update this review in order to re-evaluate the effectiveness of HFNC in the adult ICU population and incorporate the most recent evidence.

\section{O B JECTIVES}

To assess the effectiveness of HFNC compared to standard oxygen therapy, NIV or NIPPV, for respiratory support in adults in the ICU.

\section{METHODS}

\section{Criteria for considering studies for this review Types of studies}

We included all randomized controlled trials (RCTs) which used either a parallel-group or cross-over study design. Owing to the inability of randomized cross-over studies to detect long-term patient outcomes, we included this trial design only for the additional outcome measures of positive end-expiratory pressure, oxygenation, carbon dioxide clearance, respiratory rate, work of breathing, and participant-reported outcomes.

We did not impose language restrictions.

We excluded cluster-RCTs, quasi-RCTs, retrospective studies and prospective cohort or observational studies, as we wanted to focus on evidence of the highest quality from randomized studies.

\section{Types of participants}

We included studies that enrolled adults (16 years of age or older) requiring respiratory support and admitted to the ICU.

We excluded participants younger than 16 years of age. Two already published Cochrane Reviews have assessed the effectiveness of HFNC in preterm infants (Wilkinson 2016) and in the paediatric population (Mayfield 2014).

We also excluded participants not admitted to an ICU.

\section{Types of interventions}

We included humidified oxygen delivered via the nasal route at a rate greater than 20 litres per minute as the experimental intervention. We referred to this intervention as HFNC.

We compared HFNC to other types of non-invasive respiratory support which were:

- standard oxygen therapy delivered via nasal cannulae or any type of face mask with a gas flow rate of $\leq 15 \mathrm{~L} / \mathrm{min}$ (with or without humidification and heating)

- non-invasive ventilation (NIV), or non-invasive positive-pressure ventilation (NIPPV) which included devices that used bilevel positive airway pressure (BiPAP) or continuous positive airway pressure (CPAP)

We, therefore, included two distinct comparisons in the review:

- Comparison 1: HFNC versus standard oxygen therapy

- Comparison 2: HFNC versus NIV or NIPPV

\section{Types of outcome measures}

The outcome measures in this review are a mix of surrogate, clinical and participant-reported outcomes. We recognize that, while there may be a correlation between the surrogate and clinical outcomes, it is the clinical outcomes which will provide the strongest evidence regarding the safety and efficacy of HFNC. Similarly, participant- 
reported outcomes may help patients to make informed decisions about their care. We considered the short-term effects and the longterm effects of treatment, and therefore we collected outcome data up to 24 hours from the initiation of treatment (short-term) as well as at more than 24 hours (long-term).

We assessed all outcomes at the time points reported in the included studies. For participant-reported outcomes, we accepted the study authors' definitions.

\section{Important outcomes}

- Treatment failure as indicated by the need for escalation of respiratory therapy (up to 28 days). Escalation of therapy may depend on the initial type of respiratory therapy given to participants and we will be guided by study authors definitions; for example, we will include treatment failure defined as escalation from HFNC or standard oxygen therapy to NIV, NIPPV or invasive mechanical ventilation, as well as escalation from NIV or NIPPV to invasive mechanical ventilation.

- In-hospital mortality (measured up to 90 days).

- Adverse events: pneumonia and nasal mucosa or skin trauma. In comparison 2 (HFNC versus NIV or NIPPV); we also included barotrauma (pneumothorax).

- Length of ICU stay (in days).

- Short-term oxygenation: partial pressure of arterial oxygen/ fraction of inspired oxygen $\left(\mathrm{PaO}_{2} / \mathrm{FiO}_{2}\right)$ ratio $(\mathrm{mmHg})$.

- Participant-reported outcomes: short- and long-term comfort.

\section{Additional outcomes}

- Duration of any type of respiratory support (mechanical ventilation, NIPPV, HFNC, standard oxygen) (in hours);

- Long-term oxygenation: $\mathrm{PaO}_{2} / \mathrm{FiO}_{2}$ (mmHg);

- Short-term and long-term other respiratory effects as indicated by any of the following:

- Degree of atelectasis on radiological examination

- Positive end-expiratory pressure measured at the pharyngeal level $\left(\mathrm{cm} \mathrm{H}_{2} \mathrm{O}\right)$

- Oxygenation: partial pressure of oxygen in arterial blood $\left(\mathrm{PaO}_{2} ; \mathrm{mmHg}\right)$; oxygen saturation of arterial blood $\left(\mathrm{SaO}_{2}\right.$; $\mathrm{mmHg})$; and oxygen saturation $\left(\mathrm{SpO}_{2} ; \%\right)$

- Carbon dioxide clearance: partial pressure of carbon dioxide in arterial blood $\left(\mathrm{PaCO}_{2} ; \mathrm{mmHg}\right)$

- Respiratory rate (breaths per minute)

- Work of breathing (joules per litre);

- Additional adverse events: tracheobronchitis and abdominal distension;

- Length of hospital stay (in days);

- Additional short-term and long-term participant-reported outcomes as indicated by any of the following:

* Dyspnoea

* Dry mouth

* Refusal to continue with treatment:

- Cost comparison of treatment (in Australian dollars).

\section{Search methods for identification of studies}

\section{Electronic searches}

We identified RCTs through literature searching with systematic and sensitive search strategies, as outlined in Chapter 4 of the Cochrane Handbook of Systematic Reviews of Interventions (Cochrane Handbook; Higgins 2019). We applied no restrictions on language or publication status. We searched the following databases for relevant trials.

- Cochrane Central Register of Controlled Trials (CENTRAL; 2020; Issue 4);

- MEDLINE (Ovid SP; 2000 to 17 April 2020);

- Embase (Ovid SP; 2000 to 17 April 2020);

- Cumulative Index to Nursing and Allied Health Literature (CINAHL; EBSCOhost; 2000 to 17 April 2020);

- Web of Science (SCI-Expanded; 2000 to 17 April 2020);

- Cochrane COVID-19 Study Register (17 April 2020).

We restricted the search start date to 2000, as HFNC have been available for use in the adult population only since the mid-2000s. For this review update, we amended the search strategies for MEDLINE and Embase, added a search in the Cochrane COVID-19 Study Register and brought the searches in the other listed databases up to date. We also re-considered eligibility of the studies included in the previous version of the review (Corley 2017). The search strategy was developed in consultation with the Information Specialist for the Cochrane Emergency and Critical Care Group. Search strategies can be found in: Appendix 1; Appendix 2; Appendix 3; Appendix 4; Appendix 5; Appendix 6.

We searched the following clinical trials registers for ongoing and unpublished trials:

- World Health Organization International Clinical Trials Registry Platform (who.int/ictrp; on 6 April 2020);

- ClinicalTrials.gov (clinicaltrials.gov; on 6 April 2020).

\section{Searching other resources}

We carried out citation searching of identified included studies published since the last review update in Web of Science on 29 April 2020. In addition, we scanned reference lists of relevant systematic reviews which were published since 2018, and we searched Opengrey on 29 April 2020 (www.opengrey.eu).

\section{Data collection and analysis}

Two review authors (PB and SL) independently selected studies and extracted data from studies identified in the most recent search. We compared decisions at each stage, and reached consensus through discussion.

\section{Selection of studies}

We used reference management software to collate the results of searches and to remove duplicates (Endnote). We used Covidence software to screen results of the search of titles and abstracts and to identify potentially relevant studies (Covidence). We sourced the full texts of all potentially relevant studies and considered whether they met the inclusion criteria (see Criteria for considering studies for this review). 
We recorded the number of papers retrieved at each stage and reported this information in the Results.

\section{Data extraction and management}

Two review authors (PB and SL) independently extracted information and outcome data from each study using a data extraction template (Appendix 7). We compared collected information and outcome data and reached consensus through discussion.

We collected the following information.

- Methods: type of study design; setting; dates of study; funding sources and declarations of interest.

- Participants: number randomized to each group; number of losses in each group (with reasons for loss); number analysed in each group; inclusion and exclusion criteria; baseline characteristics (age, gender, body mass index, illness severity score, $\mathrm{PaCO}_{2}, \mathrm{PaO}_{2} / \mathrm{FiO}_{2}$, respiratory rate).

- Interventions: details of intervention and comparison (type of respiratory support, time of initiation, gas flow, duration of support).

- Outcomes: data for all reported outcomes to include study author definitions, measurement scales, and time points.

\section{Assessment of risk of bias in included studies}

We assessed study quality, study limitations, and the extent of potential bias using the Cochrane 'Risk of bias' tool (Higgins 2011). We considered the following domains:

- Sequence generation (selection bias);

- Allocation concealment (selection bias);

- Blinding of participants, personnel, and outcome assessors (performance and detection bias);

- Incomplete outcome data (attrition bias);

- Selective reporting (reporting bias);

- Other risks of bias.

For each domain, two review authors (PB and SL) judged whether study authors made sufficient attempts to minimize bias in their study design. For performance bias, we accepted that it was not possible to blind participants and personnel to the type or respiratory support used. For detection bias, we separated our judgements for outcomes that we considered to be subjective, which were all the participant-reported outcomes, and the other outcomes which we considered to be objective. We conducted 'Risk of bias' judgements only for studies in which we reported outcome data.

For each domain, we made judgements using three measures high, low, or unclear risk of bias. We recorded this in 'Risk of bias' tables and presented a summary risk of bias.

\section{Measures of treatment effect}

We collected dichotomous data for the primary outcomes. We collected continuous data for most of the secondary outcomes (duration of respiratory support, length of stay, and respiratory effects). We collected either continuous or dichotomous for participant-reported outcomes, such as comfort scores, depending on the methods and measurement scales used to report these outcomes in the study reports.

We reported dichotomous outcomes as risk ratios (RR) to compare groups. For continuous data, we reported the mean difference (MD). In the event that studies used different measurement scales, we scaled and inverted the scales to allow calculation of the MD where possible. Where this was not possible, we selected the standardised mean difference (SMD) for measurement. An example of scaling would include dividing the mean and standard deviation by the 10 to convert a $0-100$ scale to a 0-10 scale. An example of inverting a scale would include subtracting the mean from the highest number on the scale (i.e. 3 out of 10 on a discomfort high scale would be equivalent to 7 out of 10 on a comfort high scale). We reported $95 \%$ confidence intervals for RRs and MDs or SMDs. For outcomes for which only one study was available, we used the calculator in RevMan Web to calculate the effect estimates (RevMan Web 2019); we reported the effect estimates from single studies in additional tables. In the event that studies reported data only as median values or used scales that did not easily translate to dichotomous or continuous data, we also reported data separately in additional tables.

\section{Unit of analysis issues}

Although we included cross-over study designs, we only included data in the review if study authors reported findings for the first treatment period.

Included studies measured many of the secondary outcomes (oxygenation $\left(\mathrm{PaO}_{2}, \mathrm{PaO}_{2} / \mathrm{FiO}_{2}\right.$ ratio, $\left.\mathrm{SpO}_{2}\right)$, carbon dioxide clearance, respiratory rate, dyspnoea, mouth dryness, and patient comfort) at multiple time points. To overcome the potential for unit of analysis error, we took a simple approach to analysis of these outcomes on the advice of the statistical editor. We reported outcome data as short-term and longer-term effects, with shortterm effects resulting from initiation of therapy up to 24 hours, and longer-term effects occurring more than 24 hours after initiation of therapy. For short-term effects, we used the closest data point to 24 hours.

One study reported findings from two separate types of respiratory support (Frat 2015); in the previous version of this review an adjustment was made so that both arms could be included in the same analysis without introducing a unit of analysis issue (Corley 2017). Because we introduced two separate comparison groups in this review, such an adjustment was no longer required.

\section{Dealing with missing data}

We updated this review during the Covid-19 pandemic because the review provides relevant evidence for respiratory support in adults in the ICU. To reduce time to publication, rather than seeking additional information from all study authors, we only attempted to contact study authors to provide clarity when combining data for treatment failure; study authors did not provide additional data. We did not tabulate missing data and perform sensitivity analyses to determine the influence of missing data on effect estimates, as planned in the protocol (Corley 2012).

\section{Assessment of heterogeneity}

Using clinical judgement, we assessed participants, interventions, and outcomes for clinical heterogeneity. We assessed 
methodological heterogeneity during 'Risk of bias' assessments and by visual inspection of forest plots. We assessed statistical heterogeneity by using the $\mathrm{I}^{2}$ statistic (on a scale of $0 \%$ to $100 \%$ ) and the $\mathrm{Chi}^{2}$ test (Higgins 2019).

\section{Assessment of reporting biases}

We attempted to source the published protocols for each our included studies by using the results from our clinical trials register searches. We compared clinical trials register documents, or protocols, with published study results to assess the risk of selective reporting bias for outcomes relevant to this review. We assessed publication bias from the visual inspection of funnel plots for important review outcomes (i.e. those that we included in the 'Summary of findings' tables) if the outcomes included more than ten studies (Egger 1997).

\section{Data synthesis}

We conducted meta-analyses for outcomes for which we had comparable study data and presented a summary statistic for each outcome. We conducted analyses for outcomes using RevMan Web 2019. We performed separate analyses for comparisons of HFNC versus standard oxygen therapy and for HFNC versus NIV or NIPPV. We classified the level of heterogeneity using the $\mathrm{I}^{2}$ statistic as: $0 \%$ to $40 \%$, not important; $30 \%$ to $60 \%$, moderate heterogeneity; $50 \%$ to $90 \%$, substantial heterogeneity; and $75 \%$ to $100 \%$, considerable heterogeneity (Higgins 2019). We selected a random-effects model for all meta-analysis to account for the likely variation in the study population (Borenstein 2010).

\section{Subgroup analysis and investigation of heterogeneity}

We performed subgroup analysis for treatment failure, as indicated by the need for escalation to NIV, NIPPV or invasive ventilation, for studies in which respiratory support was given after extubation versus respiratory support that was given without prior mechanical ventilation.

\section{Sensitivity analysis}

We explored the potential effect of study limitations in the important review outcomes, as well as the effect of our chosen meta-analytical effects model. In each sensitivity analysis, we compared the effect estimate with the main analysis. We reported these effect estimates only if they indicated a difference in interpretation of the effect. We performed the following sensitivity analyses on the important review outcomes:

- We excluded studies that we judged to have a high or unclear risk of selection bias for either random sequence generation, allocation concealment, or both.

- We excluded studies that we judged to have a high risk of bias in any of the other domains (other than selection bias).
- We re-analysed the data using a fixed-effects model instead of a random-effects model.

- We excluded studies that were funded from commercial sources.

\section{Summary of findings and assessment of the certainty of the evidence}

Two review authors (PB and $\mathrm{SL}$ ) used the GRADE system to assess the certainty of the body of evidence and construct a 'Summary of findings' table associated with the following important outcomes (Guyatt 2008):

- Failure of treatment as indicated by the need for escalation of respiratory support;

- In-hospital mortality;

- Adverse events (pneumonia, nasal mucosa or skin trauma, barotrauma);

- Length of stay in days (ICU);

- $\mathrm{PaO}_{2} / \mathrm{FiO}_{2}$ ratio up to 24 hours after initiation of therapy;

- Comfort (short-term effects);

- Comfort (long-term effects).

The GRADE approach appraises the certainty of a body of evidence based on the extent to which we can be confident that an estimate of effect or association reflects the item being assessed. Evaluation of the certainty of a body of evidence considers within-study risk of bias, directness of the evidence, heterogeneity of the data, precision of the effect estimates, and risk of publication bias.

We constructed 'Summary of findings' tables using GRADEpro GDT software for the following comparisons in this review (gradepro.org):

- HFNC versus standard oxygen therapy;

- HFNC versus NIPPV or NIV.

\section{RES U L T S}

\section{Description of studies}

See Characteristics of included studies, Characteristics of excluded studies, and Characteristics of ongoing studies.

\section{Results of the search}

After the removal of duplicates from the search results, we screened 4224 titles and abstracts, which included forward and backward citation searches and searches of clinical trials registers. We looked at the full text of 138 reports and selected 31 studies for inclusion, based on review criteria. We identified 51 ongoing studies, found 19 studies for which we could not assess eligibility, and we excluded nine studies (see Figure 1). 
Figure 1. Flow diagram. Search conducted in April 2020

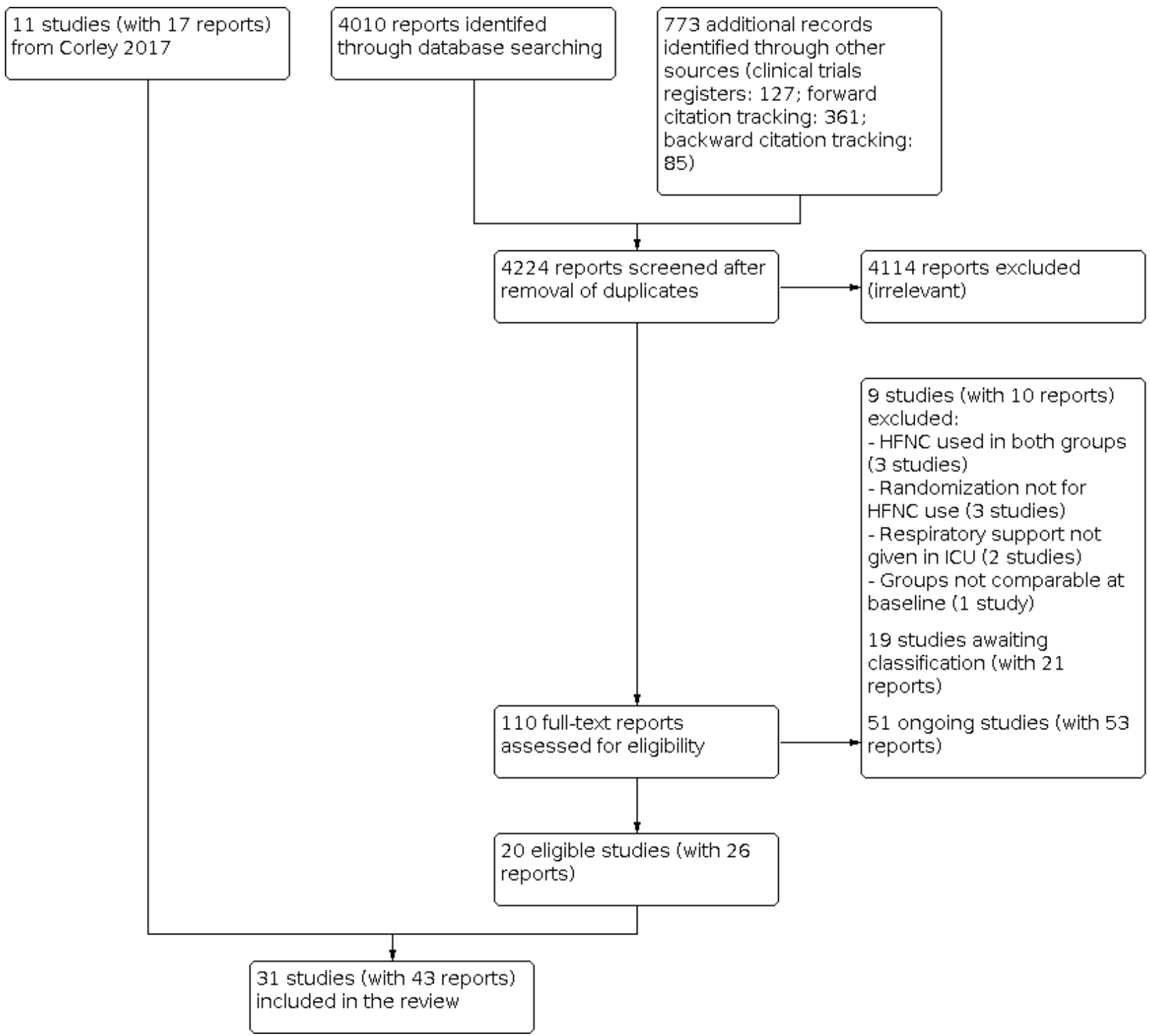

\section{Included studies}

We included 31 RCTs with 5136 randomized participants. Nine were randomized cross-over studies (Chanques 2013; Grieco 2020; Lee 2018; Longhini 2019; Mauri 2017a; Mauri 2017b; Rittayamai 2014; Schwabbauer 2014; Vargas 2015); the remaining studies all used a parallel-group design.

This update included 20 new studies (Azoulay 2018; Brainard 2017; Cong 2019; Fernandez 2017; Futier 2016; Grieco 2020; Hernandez 2016a; Hernandez 2016b; Hu 2020; Jing 2019; Lee 2018; Longhini 2019; Mauri 2017a; Mauri 2017b; Shebl 2018; Song 2017; Vargas 2015; Vourc'h 2020; Yu 2017; Zochios 2018). The remaining studies were previously included in Corley 2017.

During the previous version of the review (Corley 2017), we contacted eight study authors by email to request additional details, including outcome data not available in the published report and information for 'Risk of bias' assessment (Chanques 2013; Corley 2014; Cuquemelle 2012; Maggiore 2014; Parke 2011;
Parke 2013a; Rittayamai 2014; Schwabbauer 2014). Chanques 2013, Corley 2014, Parke 2011, Parke 2013a, and Rittayamai 2014 provided participant and outcome data and clarification on methodological issues; Cuquemelle 2012 provided information on methodological issues but was unable to provide data; Schwabbauer 2014 was unable to provide any additional details for the study. Following contact with Maggiore 2014, the full report was published, and we used data from this report, rather than information provided via email communication. In this update, we contacted two study authors by email to request additional details for our important outcomes (Fernandez 2017; Vourc'h 2020).

\section{Study population}

We included only studies that examined participants 16 years of age or older requiring respiratory support. Participants in all studies had respiratory failure or were at risk of respiratory failure. Most studies included a heterogeneous study population, with respiratory failure resulting from a variety of 
causes. Some of the studies included only participants with specific causes of respiratory failure. Three studies specifically included participants requiring support for an exacerbation of chronic obstructive pulmonary disorder (COPD) (Cong 2019; Jing 2019; Longhini 2019), and in one study all participants were immunocompromised (Azoulay 2018). Eight studies required support following cardiothoracic surgery (Brainard 2017; Corley 2014; Futier 2016; Parke 2013a; Stephan 2015; Vourc'h 2020; Yu 2017; Zochios 2018). We included only one study in which all participants had a body mass index (BMI) of at least $30 \mathrm{~kg} / \mathrm{m}^{2}$ (Corley 2014).

\section{Study setting}

All studies were conducted in intensive care units, and 11 of these were multicentre studies (Azoulay 2018; Fernandez 2017; Frat 2015; Futier 2016; Hernandez 2016a; Hernandez 2016b; Lemiale 2015; Longhini 2019; Maggiore 2014; Stephan 2015; Yu 2017).

\section{Interventions and comparisons}

All studies randomized a group of participants to receive oxygen via HFNC. We noted differences in flow rates between the studies. Most specified a range of flow rates which was between $30 \mathrm{~L} / \mathrm{min}$ and 60 L/min (Cong 2019; Cuquemelle 2012; Fernandez 2017; Lee 2018; Maggiore 2014; Parke 2011; Parke 2013a; Rittayamai 2014; Yu 2017; Zochios 2018); in one study, this range had a lower flow rate of 20 to $50 \mathrm{~L} / \mathrm{min}$ (Futier 2016). Others specified an initial flow rate, with subsequent increases upwards from $10 \mathrm{~L} /$ min (Hernandez 2016a), or decreases from $60 \mathrm{~L} / \mathrm{min}$ (Song 2017). Twelve studies specified a maximum target of up to $50 \mathrm{~L} / \mathrm{min}$ (Corley 2014; Frat 2015; Grieco 2020; Lemiale 2015; Stephan 2015), or up to 60 L/min (Azoulay 2018; Hu 2020), or a set flow rate of $40 \mathrm{~L} / \mathrm{min}$ (Brainard 2017; Mauri 2017b), $45 \mathrm{~L} / \mathrm{min}$ (Vourc'h 2020), $55 \mathrm{~L} / \mathrm{min}$ (Schwabbauer 2014), or $60 \mathrm{~L} /$ min (Vargas 2015). Two multi-arm studies tested oxygen delivery at different flow rates (Chanques 2013; Mauri 2017a); these were 15,30 , and $45 \mathrm{~L} / \mathrm{min}$, and at 30,45 , and $60 \mathrm{~L} / \mathrm{min}$, respectively. Flow rates were not specified in four studies (Jing 2019; Lee 2018; Longhini 2019; Shebl 2018).

Three multi-arm studies included control groups for both of our comparison groups (Chanques 2013; Frat 2015; Schwabbauer 2014). In the standard oxygen therapy comparison group, most studies had a control group in which oxygen was delivered with face masks using a simple face mask, nasal cannulae, a non-rebreather face mask or Venturi mask; in Chanques 2013, the control used a high-flow face mask, and in Parke 2011 and Vourc'h 2020, they used a high-flow face mask with humidifier. Cuquemelle 2012 described the use of 'standard oxygen therapy', with no additional details.

In the NIV and NIPPV comparison group, delivery devices were via Bossignac oxygen therapy (Chanques 2013), and bilevel positive airway pressure (BiPAP) (Cong 2019; Frat 2015; Grieco 2020; Hernandez 2016a; Jing 2019; Schwabbauer 2014; Stephan 2015; Shebl 2018; Vargas 2015).

Fourteen studies initiated the intervention or control after extubation from invasive mechanical ventilation (Chanques 2013; Corley 2014; Fernandez 2017; Futier 2016; Hernandez 2016a; Jing 2019; Maggiore 2014; Parke 2013a; Rittayamai 2014; Song 2017; Stephan 2015; Vourc'h 2020; Yu 2017; Zochios 2018). Participants in Futier 2016, Yu 2017 and Zochios 2018 were at high risk of pulmonary complications. Participants in Hernandez 2016a and Hu 2020 were at high risk of extubation failure, and low risk of extubation failure in Hernandez 2016b. The remaining studies initiated the intervention without previously using mechanical ventilation.

\section{Outcomes}

For cross-over studies, we only included outcome data from the first treatment period. This was reported in only three of the crossover studies (Chanques 2013; Cuquemelle 2012; Rittayamai 2014), and we, therefore, did not report data for the remaining cross-over studies. In Cuquemelle 2012, which included a four-hour cross-over period at the end of a 24-hour parallel-group assignment period, we included only narrative results from the initial 24-hour period. For the remaining studies, we reported outcome data as specified in the study reports.

\section{Funding}

Whilst some studies were supported by funding that we considered to be independent of the study (for example, university or government health ministries), we noted that 14 studies were supported by grants or by the provision of study equipment by manufacturers (Fisher \& Paykel Healthcare (Azoulay 2018; Chanques 2013; Corley 2014; Cuquemelle 2012; Frat 2015; Hernandez 2016b; Lemiale 2015; Maggiore 2014; Mauri 2017b; Parke 2011; Parke 2013a; Schwabbauer 2014; Zochios 2018)) and from Merck Sharp \& Dohome (Grieco 2020). Most studies declared that these manufacturers were not involved in the design or conduct of the study, nor in the interpretation of the results or preparation of the final manuscripts for publication. However, in Parke 2011, study authors declared that Fisher \& Paykel Healthcare were involved in the study design and data analysis, and provided financial support for the statistical analysis.

\section{Excluded studies}

We excluded nine studies during full-text review (Coudroy 2019; Delorme 2017; Di Mussi 2016; Lemiale 2016; Liu 2019; Pennisi 2019; Sklar 2018; Thille 2018; Thille 2019). We excluded these nine studies owing to the use of HFNC in both the intervention and control arms of the study (Coudroy 2019; Thille 2018; Thille 2019), because participants receiving HFNC or standard oxygen therapy were not randomized to this treatment (Delorme 2017; Di Mussi 2016; Lemiale 2016), and because the study setting was not in an ICU (Pennisi 2019; Sklar 2018). See Characteristics of excluded studies.

This review does not include studies that were previously excluded; details of previous exclusions can be found in the earlier version of the review (Corley 2017).

\section{Studies awaiting classification}

We were unable to assess eligibility for 19 studies (Arman 2017; Guoqiang 2018; Gupta 2016; Ischaki 2019; ISRCTN17399068; Lee 2016; Longhini 2017; Macari 2019; Menga 2019; Papachatzakis 2017; Perbet 2014; Saeed 2015; Schreiber 2017; Theerawit 2017; Tseng 2019; Yang 2019; Zhang 2018; Zhao 2019; Zhu 2017); this included two studies that were awaiting classification in a previous version of this review (Perbet 2014; Saeed 2015).

Four of these studies were not published in English and required translation before inclusion in the review (Yang 2019; Zhang 2018; Zhao 2019; Zhu 2017). We identified one completed study in the clinical trial register searches, but because the data in the clinical 
trials register had not been peer-reviewed, we did not include it in the review (ISRCTN17399068). The remaining studies were published as abstracts; we are awaiting publication of the full study reports for these studies (and for the study in the clinical trials report) in order to fully assess eligibility and incorporate these results in the review.

Eight of the studies awaiting classification investigated HFNC for post-extubation respiratory support; of these, two included participants with acute exacerbations of COPD (Guoqiang 2018; Zhang 2018), one included participants post-surgery (Gupta 2016), and five considered all intubated participants in an ICU setting (Arman 2017; Perbet 2014; Theerawit 2017; Tseng 2019; Zhu 2017). Ten studies investigated HFNC for respiratory support without prior use of invasive mechanical ventilation; of these, five included participants with acute exacerbations of COPD (Ischaki 2019; Lee 2016; Longhini 2017; Saeed 2015; Yang 2019), and five included participants with acute hypoxic respiratory failure (AHRF) (Macari 2019; Menga 2019; Papachatzakis 2017; Schreiber 2017; Zhao 2019).

\section{Ongoing studies}

We identified 51 ongoing RCTs with an estimated recruitment of 12,807 participants (see Characteristics of ongoing studies). Three studies marked as ongoing in the previous version of this review had been completed and are now included in this update (Fernandez 2017; Vargas 2015; Vourc'h 2020). Five of the 51 studies were cross-over RCTs (NCT03811158; NCT03865056; NCT03877172; NCT04036175; NCT04241861), one was a $2 \times 2$ factorial design RCT (NCT04344730), and the remaining studies were parallel-group design RCTs.

Six studies, with an estimated 4802 participants, had more than two study arms, and compared HFNC to both standard oxygen therapy and NIV or NIPPV (CTRI/2018/09/015717; ChiCTR-INR-17012720; ISRCTN16912075; NCT03171935; NCT03229460; NCT04269681). Twelve studies, with an estimated 1780 randomized participants, compared HFNC to standard oxygen therapy (ACTRN12617000694314; ChiCTR1900021091; NCT01702779; NCT02107183; NCT02290548; NCT03133520; NCT03282552; NCT03361683; NCT03430258; NCT03515031; NCT03811158; NCT03877172). The remaining studies, with an estimated 6225 randomized participants, compared HFNC to NIV or NIPPV.

One study investigated HFNC for both post-extubation respiratory support and respiratory support without prior use of mechanical ventilation (NCT04269681). Twenty-three studies investigated
HFNC for post-extubation respiratory support. Of these 23 studies, five included participants with no criteria other than intubation for: at least 24 hours (NCT02107183; NCT04036175); at least 48 hours (NCT01702779; NCT02123940); or with pre-existing respiratory disease (NCT03632577). Six studies included participants with acute exacerbations of COPD (ChiCTR-INR-17011850; ChiCTRINR-17012720; ChiCTR1900025974; NCT02290548; NCT03811158; NCT04156139), five included participants after surgery that had a high risk of extubation failure (ACTRN12617000694314; NCT02713737; NCT03282552; NCT03877172; NCT03928535), two included participants with pneumonia (ChiCTR1900020826; ChiCTR1900021091), four included participants with AHRF (ChiCTR1900023296; NCT02290548; NCT03171935; NCT03361683), one included participants with trauma and acute respiratory distress syndrome (ChiCTR1900023296), one included participants with heart failure (NCT03607357), and one included participants with sepsis (NCT03246893).

Twenty-seven studies investigated HFNC for respiratory support without prior uses of mechanical ventilation. Of these, 20 included participants with AHRF (CTRI/2018/09/015717; ChiCTR1800017313; ChiCTR1900022241; JPRN-jRCTs052180236; NCT01166256; NCT02464696; NCT03133520; NCT03229460; NCT03488628; NCT03643939; NCT03788304; NCT03865056; NCT03944525; NCT04035460; NCT04241861; NCT04253405; NCT04293991; NCT04344730; TCTR20171106003; UMIN000008778), five included participants with acute exacerbations of COPD (ChiCTR1800014553; ChiCTR1800018530; Cortegiani 2019; NCT03014869; NCT03643939), two included participants with traumatic injuries (ChiCTR1800017313; NCT03430258), three included participants with pneumonia (ISRCTN16912075; NCT03515031; NCT04344730), of which two focused specifically on patients with COVID-19 disease (ISRCTN16912075; NCT04344730).

\section{Risk of bias in included studies}

We described the risk of bias for each included study in the 'Risk of bias' tables in Characteristics of included studies (see Figure 2). We did not conduct risk of bias assessment for cross-over studies in which data were not reported for the first period; the 'Risk of bias' figure, therefore, includes blank spaces for seven studies (Grieco 2020; Lee 2018; Longhini 2019; Mauri 2017a; Mauri 2017b; Schwabbauer 2014; Vargas 2015). In addition, we assessed risk of detection bias separately for subjective and objective outcome measures; therefore, some blank spaces in the risk of bias figure indicate that we did not assess risk of bias because the study did not report either objective or subjective outcomes relevant to the review.

Figure 2. Risk of bias summary: review authors' judgements about each risk of bias item for each included study. We only conducted 'Risk of bias' assessments in studies for which we reported outcome data, and for domains that were relevant to reported outcomes (in particular, for detection bias of objective and subjective measures); blank 
spaces, therefore, indicate that 'Risk of bias' assessment was not conducted for the outcome, or for a particular domain.

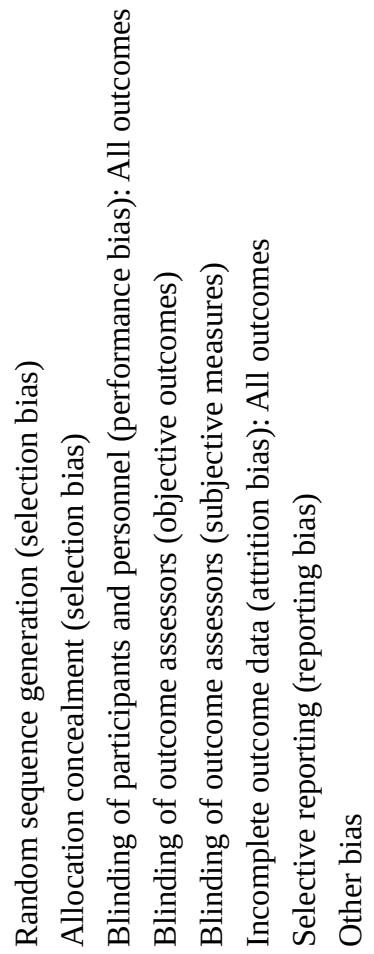

Azoulay 2018

Brainard 2017

Chanques 2013

Cong 2019

Corley 2014

Cuquemelle 2012

Fernandez 2017

Frat 2015

Futier 2016

Grieco 2020

Hernandez 2016a

Hernandez 2016b

Hu 2020

Jing 2019

Lee 2018

Lemiale 2015

Longhini 2019

Maggiore 2014

Mauri 2017a

Mauri 2017b

Parke 2011

Parke 2013a

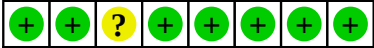

\begin{tabular}{|l|l|l|l|l|l|l|l|}
\hline$?$ & $?$ & $?$ & + & + & $?$ & $?$ & + \\
\hline
\end{tabular}

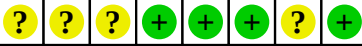
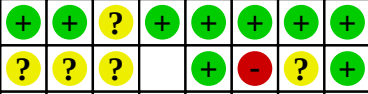

$++?$

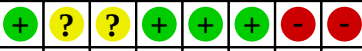

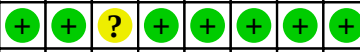

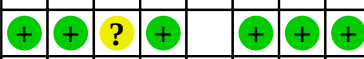

++ ?

\begin{tabular}{|l|l|l|l|l|l|l|l|}
\hline+ & ? & ? & + & & + & - & + \\
\hline
\end{tabular}

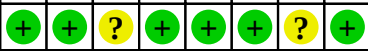

\begin{tabular}{llllllll}
\hline+ & + & + & + & + & + & $?$ & +
\end{tabular}

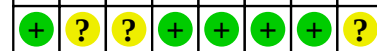

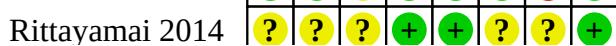


Figure 2. (Continued)

\begin{tabular}{|c|c|c|c|c|c|c|c|c|}
\hline Parke 2013a & + & + & ? & + & + & + & & \\
\hline Rittayamai 2014 & ? & $?$ & $?$ & + & + & $?$ & $?$ & + \\
\hline \multicolumn{9}{|l|}{ Schwabbauer 2014} \\
\hline Shebl 2018 & ? & ? & ? & + & & + & $?$ & + \\
\hline Song 2017 & + & ? & ? & + & + & + & $?$ & + \\
\hline Stephan 2015 & + & $?$ & $?$ & + & + & + & + & + \\
\hline Vargas 2015 & & & & & & & & \\
\hline Vourc'h 2020 & + & + & $?$ & + & + & + & $?$ & + \\
\hline Yu 2017 & + & $?$ & $?$ & + & + & + & $?$ & + \\
\hline Zochios 2018 & + & + & $?$ & + & + & + & & + \\
\hline
\end{tabular}

\section{Allocation}

We found that six studies did not adequately describe a method used to randomize participants to groups (Brainard 2017; Chanques 2013; Cong 2019; Cuquemelle 2012; Rittayamai 2014; Shebl 2018); it was, therefore, unclear whether these studies were at risk of selection bias. We judged the remaining studies to be at low risk of selection bias for random sequence generation because they reported using an appropriate method such as a block system or a computer-generated sequence.

We judged only 11 studies to be at low risk of selection bias for using a method for concealing allocation (Azoulay 2018; Corley 2014; Fernandez 2017; Futier 2016; Hernandez 2016a; Hernandez 2016b; Jing 2019; Lemiale 2015; Parke 2013a; Vourc'h 2020; Zochios 2018).

\section{Blinding}

Owing to the nature of the intervention and comparators, it was not possible to blind participants and their treating clinicians to treatment allocation. Although we believed that knowledge of treatment would not influence performance for the outcomes of interest for this review, we could not be certain of this and we therefore judged all studies to have unclear risk of performance bias.

We judged risk of detection bias according to whether outcomes were objective or subjective. We defined the subjective measures as those being assessed by the participants; these outcomes were dyspnoea, comfort, dry mouth, and refusal to continue with treatment. We defined the remaining outcomes as objective, and we anticipated that knowledge of treatment allocation would not influence the assessment of these outcomes. Therefore, we judged all studies that reported objective measures to have a low risk of detection bias.

Fifteen studies reported subjective measures (Azoulay 2018; Chanques 2013; Cong 2019; Corley 2014; Cuquemelle 2012; Frat 2015; Futier 2016; Jing 2019; Lemiale 2015; Maggiore 2014; Parke 2013a; Rittayamai 2014; Song 2017; Stephan 2015; Vourc'h 2020). However, we believe that the inability to blind participants to treatment allocation would not affect outcome measurements because it would be unlikely that participants would have a particular bias towards one medical intervention over another. We, therefore, judged these studies to have a low risk of detection bias for subjective measures.

\section{Incomplete outcome data}

We judged two studies to have a high risk of attrition bias because a large number of participants in these studies were not included in analysis (Brainard 2017; Cuquemelle 2012). In three additional studies, we could not be certain whether participants were lost to follow-up, or how the data were managed when participants were treated with an alternative therapy (Chanques 2013; Parke 2011; Rittayamai 2014); in these studies, we judged risk of attrition bias to be unclear. The remaining studies reported no or few losses that were sufficiently explained, and we judged these studies to have a low risk of attrition bias.

\section{Selective reporting}

Eleven studies reported clinical trials registration which was made prospectively (Azoulay 2018; Corley 2014; Fernandez 2017; Frat 2015; Futier 2016; Hernandez 2016a; Hernandez 2016b; Hu 2020 ;Parke 2011; Parke 2013a; Zochios 2018). However, only five of these prospectively registered studies reported outcomes that were consistent with the clinical trials register documents (Azoulay 2018; Corley 2014; Futier 2016; Hernandez 2016a; Hernandez 2016b). We assessed six of these to be at high risk of bias because they either reported outcomes that were not listed in the clinical trials register documents, or failed to report outcomes as specified in the clinical trials register documents (Fernandez 2017; Frat 2015; Hu 2020; Parke 2011; Parke 2013a; Zochios 2018). We made these risk of bias judgements only according to the outcomes that were relevant to this review.

Six studies reported clinical trials registration which was made retrospectively (Brainard 2017; Chanques 2013; Jing 2019; Lemiale 2015; Maggiore 2014; Stephan 2015). We assessed Maggiore 2014 and Stephan 2015 to be at low risk of selective reporting bias because they were registered only shortly after the study start date, and study authors reported the same outcome data as in the clinical trials register documents. However, we did not think it was feasible to effectively assess risk of selective reporting bias for the remaining retrospectively registered studies, and we judged bias in these studies to be unclear.

The remaining studies did not report clinical trials registration, nor reported a protocol published prior to the completed study report, and we judged risk of selective reporting bias to be unclear (Cong 2019; Cuquemelle 2012; Rittayamai 2014; Shebl 2018; Song 2017; Vourc'h 2020; Yu 2017). 


\section{Other potential sources of bias}

To date, one of the included studies (Maggiore 2014) has presented three abstracts, and this study is part of a larger ongoing clinical trial (see NCT02107183 in Characteristics of ongoing studies); multiple interim analyses could introduce bias (Bland 1995), and we judged risk of other bias in this study to be unclear.

In Frat 2015, we noted that participants in the NIPPV group could have been exposed to HFNC during breaks in delivery of oxygen, during which choice of oxygen delivery was at the discretion of the attending clinician. Because some participants in the NIPPV group could have received HFNC, we judged this study to have high risk of bias as a result of this methodological decision.

We identified no other sources of bias in the remaining studies.

\section{Effects of interventions}

See: Summary of findings 1 HFNC compared to standard oxygen therapy for respiratory support in adult intensive care patients; Summary of findings 2 HFNC compared to NIPPV or NIV for respiratory support in adult intensive care patients

See Summary of findings 1.

\section{Comparison 1: HFNC versus standard oxygen therapy}

\section{Important outcomes}

Failure of treatment as indicated by escalation of respiratory therapy to NIV, NIPPV or invasive ventilation

Sixteen studies reported failure of treatment indicated by escalation to alternative oxygen therapy (Azoulay 2018; Corley
2014; Cuquemelle 2012; Fernandez 2017; Frat 2015; Futier 2016; Hernandez 2016b; Hu 2020; Lemiale 2015; Maggiore 2014; Parke 2011; Parke 2013a; Song 2017; Vourc'h 2020; Yu 2017; Zochios 2018). In studies that separately reported escalation to different devices, we sought contact with authors to establish that participants were not counted more than once for use of each type of support (Fernandez 2017; Vourc'h 2020). Two studies did not separately report data according to type of escalation, and we included combined data for escalation to re-intubation or NIV in Futier 2016, and to NIV, or intubation in Zochios 2018. For Hu 2020, we included data that was for escalation to NIV.

We did not include data from Cuquemelle 2012 in analysis, because we could not confirm the time point of treatment failure; study authors reported less treatment failure associated with HFNC, with one of 19 participants in the HFNC group failing treatment compared with four of 18 participants in the low-flow oxygen group.

We found that using HFNC may lead to less treatment failure indicated by escalation to alternative types of oxygen therapy (risk ratio (RR) $0.62,95 \%$ confidence interval $(\mathrm{Cl}) 0.45$ to $0.86 ; 15$ studies, 3044 participants; $1^{2}=72 \%$; low-certainty evidence; Analysis 1.1 ). We generated a funnel plot for this evidence and, from visual inspection of this plot, we noted no evidence of publication bias (Figure 3). 
Figure 3. Funnel plot for outcome 1.1 Treatment failure.

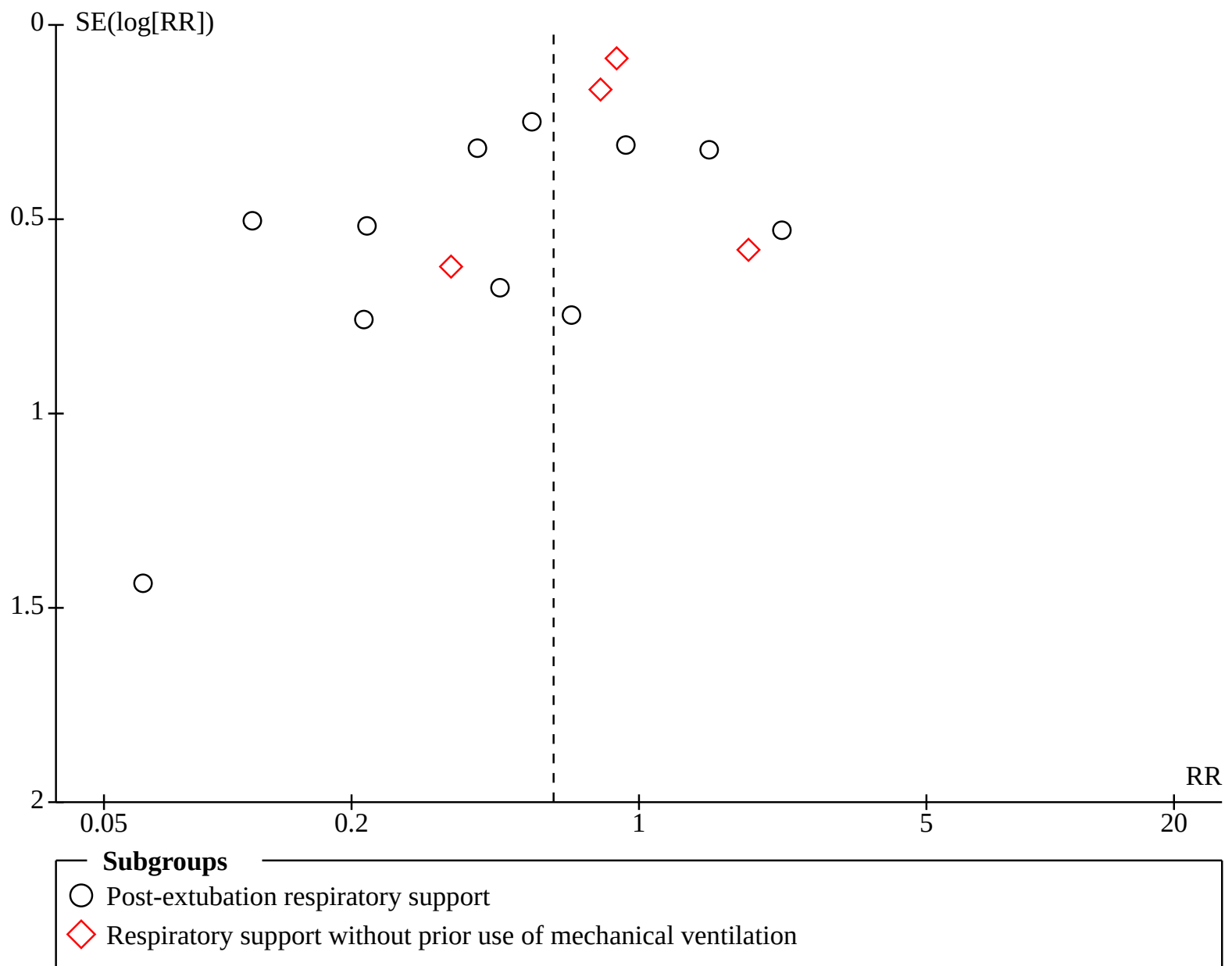

We used GRADE to downgrade the certainty of the evidence by two levels. We downgraded by one level for inconsistency because we noted a moderate level of statistical heterogeneity; we also noted more variation in the data between studies in which respiratory support was given post-extubation which we could not explain. We also downgraded by one level for study limitations because we judged some studies to have an unclear risk bias; excluding these studies in sensitivity analysis no longer indicated an improvement with HFNC use (see Summary of findings 1 ).

\section{In-hospital mortality}

Eleven studies reported mortality up to 90 days (Azoulay 2018; Fernandez 2017; Frat 2015; Futier 2016; Hernandez 2016b; Hu 2020;
Maggiore 2014; Parke 2013a; Vourc'h 2020; Yu 2017; Zochios 2018). As well as data specified as in-hospital, we included data in analysis in which time points were up to ICU discharge (Frat 2015; Maggiore 2014), up to day 28 days (Parke 2013a), or at nonspecified time points (Yu 2017).

We found no evidence of a difference in mortality when HFNC was compared to standard oxygen therapy (RR $0.96,95 \% \mathrm{Cl} 0.82$ to 1.11 ; 11 studies, 2673 participants; $12=0 \%$; moderate-certainty evidence; Analysis 1.2). We generated a funnel plot for this evidence and, from visual inspection of this plot, we noted no evidence of publication bias (Figure 4). 
Figure 4. Funnel plot for outcome 1.2 In-hospital mortality

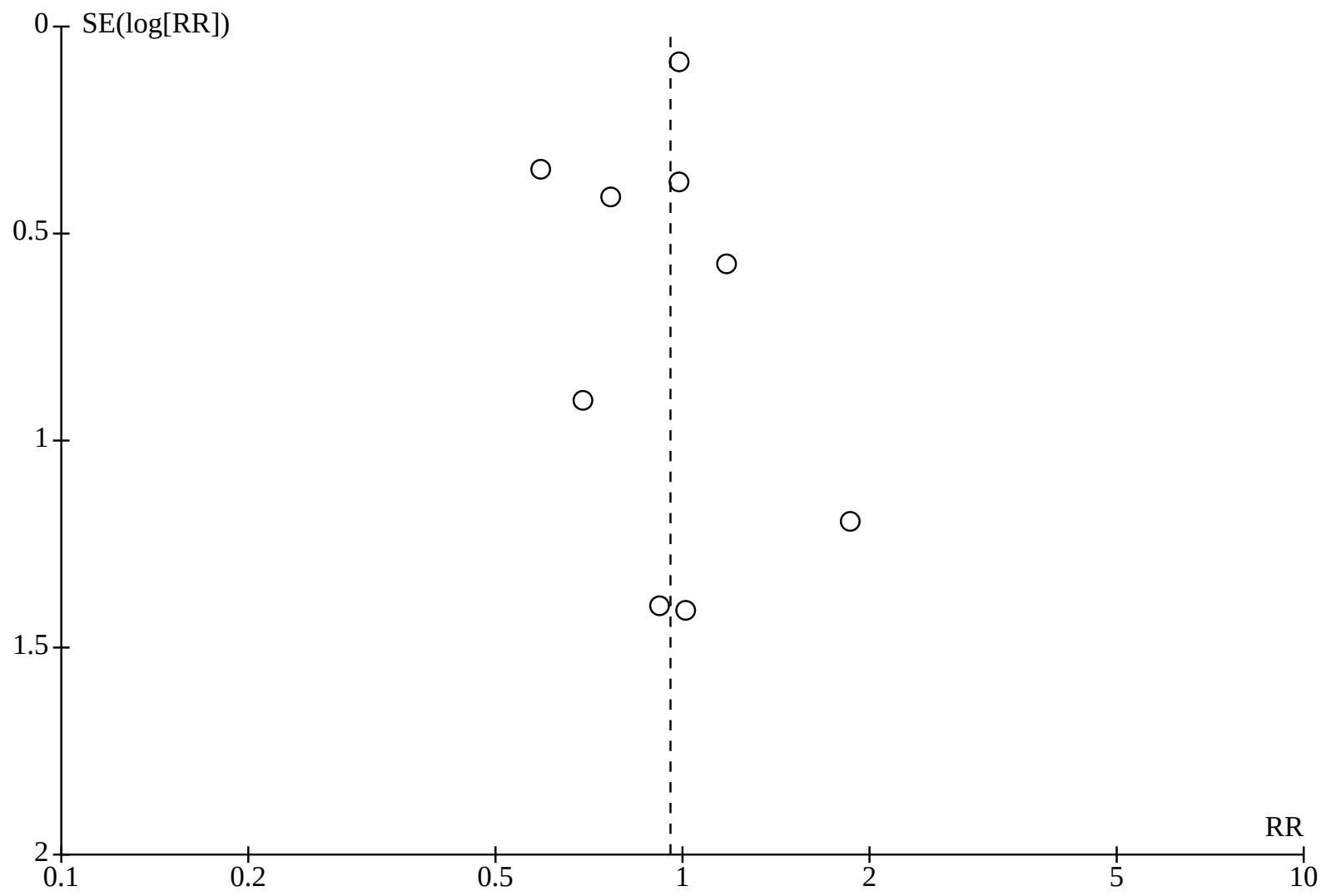

We used GRADE to downgrade the certainty of the evidence by one level for inconsistency. We noted inconsistencies in the data, which we expected were caused by the differences in the severity of illness of participants in the studies which is likely to impact on mortality rates between studies (see Summary of findings 1 ).

\section{Important adverse events}

\section{Pneumonia}

Four studies reported the number of people who acquired suspected or confirmed pneumonia (Frat 2015; Futier 2016; Hernandez 2016b; Yu 2017). We found that HFNC may result in little or no evidence of a difference in cases of pneumonia according to the method of respiratory support (RR $0.72,95 \% \mathrm{Cl} 0.48$ to 1.09 ; 4 studies, 1057 participants; $\left.\right|^{2}=0 \%$; moderate-certainty evidence; Analysis 1.3). We used GRADE to downgrade the certainty of the evidence by one level for imprecision because only four studies contributed evidence for this outcome (see Summary of findings 1 ).

\section{Nasal mucosa or skin trauma}

Two studies reported incidences of nasal mucosa or skin trauma (Hernandez 2016b; Vourc'h 2020). We found no evidence of difference in nasal mucosa or skin trauma (RR 3.66, 95\% Cl 0.43 to 31.48 ; 2 studies, 617 participants; $1^{2}=0 \%$; very lowcertainty evidence; Analysis 1.3); in Hernandez 2016b, study authors reported no events. We used GRADE to downgrade the certainty of the evidence by two levels for imprecision because only two studies contributed evidence, of which only one reported events, and by one level for study limitations because this study did not report study trials registration and we could not be certain whether it was at risk of selective reporting bias (see Summary of findings 1$)$.

\section{Length of ICU stay}

Twelve studies reported length of stay in the ICU (Azoulay 2018; Brainard 2017; Corley 2014; Frat 2015; Futier 2016; Hernandez 2016b; Hu 2020; Maggiore 2014; Parke 2013a; Vourc'h 2020; Yu 2017; Zochios 2018).

We combined data in which values were reported as mean length of stay and found no evidence of a difference in the mean number of days according to the type of respiratory support used (MD 0.12 days, $95 \% \mathrm{Cl}-0.03$ to $0.27 ; 7$ studies, 1014 participants; $12=0 \%$; low-certainty evidence; Analysis 1.4). Frat 2015 reported length of stay calculated at 90 days separately for both survivors and nonsurvivors; in meta-analysis, we included only the data for survivors because this included data for a larger number of participants, but we note that data for non-survivors indicated a longer length of ICU stay. From visual inspection, we noted that these data were likely to be right-skewed due to the comparable magnitudes of the mean and standard deviation. This is expected for outcomes such a length of ICU stay due to most participants being discharged in a short time period with some outliers staying significantly longer. However, right skew introduces artefact into calculation of the effect estimate, limiting the interpretation of the result.

Five studies reported data as median values and we did not include these data in the analysis (Azoulay 2018; Futier 2016; Hernandez 
2016b; Hu 2020; Zochios 2018). Each study found little or no difference in the median number of days in the ICU according to the method of respiratory support that participants received (Table 1).

We used GRADE to downgrade the certainty of the evidence for this outcome to low. We downgraded by one level for inconsistency because we noted variation in the lengths of stay between studies which we expected was because of different illness severities between study participants, and by one level for study limitations because we noted some high risks of bias in some included studies. See Summary of findings 1.

\section{Short-term oxygenation $\left(\mathrm{PaO}_{2} / \mathrm{FiO}_{2}\right)$}

Eight studies reported the ratio of partial pressure of arterial oxygen to the fraction of inspired oxygen up to 24 hours (Azoulay 2018; Corley 2014; Frat 2015; Hernandez 2016b; Maggiore 2014; Parke 2011; Vourc' $h$ 2020; Yu 2017). We did not include data for Hernandez 2016b and Yu 2017 in the review; in Hernandez 2016b, these were reported only for those who were re-intubated or had postextubation respiratory failure, and in Yu 2017, these were presented in figures which we could not clearly translate into numerical data. We found no evidence of a difference in $\mathrm{PaO}_{2} / \mathrm{FiO}_{2}$ up to 24 hours according to type of respiratory support used (MD $10.34 \mathrm{mmHg}$, $95 \% \mathrm{Cl}-17.31$ to $38 ; 5$ studies, 600 participants; $\mathrm{I}^{2}=83 \%$; very lowcertainty evidence; Analysis 1.5); however, we noted substantial statistical heterogeneity in this effect. This analysis did not include Azoulay 2018 in which data were reported in median values; study authors described a higher $\mathrm{PaO}_{2} / \mathrm{FiO}_{2}$ for participants who were treated using HFNC (see Table 1 ).

We used GRADE to downgrade the certainty of the evidence to very low. We downgraded by two levels for inconsistency because we noted a substantial amount of statistical heterogeneity which we could not explain, and we downgraded by one level for study limitations because we noted differences in the effect estimate when we excluded studies at unclear or high risk of bias in sensitivity analyses (see Summary of findings 1 ).

\section{Comfort}

Six studies reported comfort scores up to 24 hours (Frat 2015; Lemiale 2015; Maggiore 2014; Parke 2013a; Rittayamai 2014; Song 2017).

The terminology and the scale of measurement differed between studies. However, it was feasible to combine data in four studies, using the highest number on the scale to indicate most comfort (Frat 2015; Maggiore 2014; Parke 2013a; Rittayamai 2014); in Frat 2015; Maggiore 2014; Rittayamai 2014, we inverted the mean scores to make higher scores represent more comfort; in Frat 2015 we also scaled mean and standard deviation on the 0 - 100 scale to a $0-10$ scale by dividing the mean and SD by 10 . In studies that reported different time points for measures of comfort, we selected time points at one hour (Maggiore 2014), four hours (Parke 2013a) and 30 minutes (Rittayamai 2014). We found no evidence of a difference in comfort according to the type of respiratory support used (MD $0.31,95 \% \mathrm{Cl}-0.60$ to $1.22 ; 4$ studies, 662 participants; $\mathrm{I}^{2}=$ $79 \%$; very low-certainty evidence; Analysis 1.6). We did not include data for Lemiale 2015 and Song 2017, because these data were reported as median values. In Lemiale 2015, study authors reported no statistically significant differences between groups measured at
120 minutes $(P=0.88)$ and in Song 2017 , study authors reported improved comfort with the use of HFNC ( $P=0.003)$ (see Table 1 ).

We used GRADE to downgrade the certainty of the evidence by three levels to very low. We downgraded by two levels for inconsistency because we noted a substantial level of statistical heterogeneity and variation in the data between the studies which we could not explain, and we downgraded by one level for imprecision because few studies contributed data for this outcome (see Summary of findings 1$)$.

Three studies also reported comfort scores at more than 24 hours (Azoulay 2018; Maggiore 2014; Parke 2013a). In Azoulay 2018, data were reported in a figure which we could not clearly translate into numerical values; study authors reported that comfort scores were not significantly different between groups. We found no evidence of a difference in comfort scores according to the type or respiratory support in the remaining studies (MD $0.59,95 \% \mathrm{Cl}-2.29$ to 3.47 ; 2 studies, 445 participants; $1^{2}=96 \%$; very low-certainty evidence; Analysis 1.6).

We used GRADE to downgrade the certainty of the evidence by three levels to very low. We downgraded by two levels for inconsistency because we noted a substantial level of statistical heterogeneity and variation in the data between the studies which we could not explain, and we downgraded by one level for imprecision because few studies contributed data for this outcome (see Summary of findings 1$)$.

\section{Additional outcomes}

We found no evidence of a difference in most additional outcomes (duration of respiratory support; long-term $\mathrm{PaO}_{2} / \mathrm{FiO}_{2}$; atelectasis, short-term $\mathrm{PaO}_{2}$; short- and long-term $\mathrm{SpO}_{2}$ and $\mathrm{PaCO}_{2}$; long-term respiratory rate; adverse events (ventilator-acquired tracheobronchitis and abdominal distension); length of hospital stay; participant-reported outcomes (dyspnoea, throat or nasal pain, treatment withdrawn due to discomfort, refusal to continue treatment); and cost comparison). We noted an effect on the following outcomes:

- Long-term $\mathrm{PaO}_{2}$ : we found higher $\mathrm{PaO}_{2}$ at more than 24 hours for participants who were treated using HFNC (MD $12.27 \mathrm{mmHg}$, $95 \% \mathrm{Cl} 7.51$ to $17.04 ; 2$ studies, 644 participants; $\left.\right|^{2}=0 \%$; Analysis 1.8).

- Long-term $\mathrm{SpO}_{2}$ : we found higher $\mathrm{SpO}_{2}$ at more than 24 hours for participants who were treated using HFNC (MD 1.28 \%, 95\% $\mathrm{Cl} 0.02$ to $2.55 ; 2$ studies, 445 participants; $\mathrm{I}^{2}=81 \%$ ). We noted substantial statistical heterogeneity in this effect. Although the effect was statistically significant $(P=0.05)$, the high number of comparisons in this review limits our interpretation of this result.

- Short-term respiratory rate: we found that the respiratory rate was improved when oxygen was delivered using HFNC (MD -2.02, $95 \% \mathrm{Cl}-3.66$ to $-0.37 ; 7$ studies, 1017 participants; $\left.\right|^{2}=87 \%$; Analysis 1.11); however, we noted substantial heterogeneity in this effect.

- Dry mouth: we found that fewer people experienced mouth dryness when HFNC was used; we did not combine data from two studies because data were reported differently (RR 0.55 , $95 \% \mathrm{Cl} 0.36$ to $0.83 ; 1$ study, 90 participants; Table 2; and MD $-1.40,95 \% \mathrm{Cl}-2.68$ to -0.12 ; 1 study, 105 participants; Table 1 ). 
- Refusal to continue with treatment: we found that more people refused to continue with treatment owing to excess heat or discomfort (RR 40.52, $95 \% \mathrm{Cl} 2.47$ to $664.65 ; 1$ study, 340 participants; Table 2).

We reported the summary effects of additional outcomes in Table 3. No studies reported useable outcome data for PEEP, $\mathrm{SaO}_{2}$, and work of breathing.

\section{Subgroup analysis}

We performed subgroup analysis for treatment failure according to whether participants were treated post-extubation following invasive mechanical ventilation, or whether therapy had not previously included mechanical ventilation.

We found no evidence of a difference between subgroups ( $P=0.07)$ (Analysis 1.1).

\section{Sensitivity analysis}

We performed sensitivity analyses on the 'important outcomes', and presented a summary of these analyses in Table 4. Whilst most sensitivity analyses did not alter our interpretation of the effects, we noted a difference in effect for the following:

- Failure of treatment: when excluding studies at high or unclear risk of selection bias, we noted that the effect estimate no longer indicated improvement with HFNC use (RR 0.85, 95\% Cl 0.62 to 1.17; 9 studies, 2457 participants; $\left.\right|^{2}=55 \%$ ).

- $\mathrm{PaO}_{2} / \mathrm{FiO}_{2}$ up to 24 hours: when excluding studies at high or unclear risk of selection bias, we noted that the effect estimate indicated higher $\mathrm{PaO}_{2} / \mathrm{FiO}_{2}$ when standard oxygen therapy was used (MD $25.28 \mathrm{mmHg}, 95 \% \mathrm{Cl} 7.23$ to 43.32; 2 studies, 245 participants; $12=0 \%$ )

- $\mathrm{PaO}_{2} / \mathrm{FiO}_{2}$ up to 24 hours: when excluding studies at high risk of attrition bias, selective reporting bias and other bias, we noted that the effect estimate indicated higher $\mathrm{PaO}_{2} / \mathrm{FiO}_{2}$ when standard oxygen therapy was used (MD $29.28 \mathrm{mmHg}, 95 \% \mathrm{Cl}$ 13.86 to $44.70 ; 3$ studies, 350 participants; $12=0 \%$ ).

- Comfort (long-term): when excluding studies at high risk of selective reporting bias, we noted that the effect estimate indicated improved comfort when HFNC was used (MD 2.10, 95\% $\mathrm{Cl} 1.04$ to 3.16 ; 1 study, 105 participants); however this effect included data from only one study.

\section{Comparison 2: HFNC versus NIPPV or NIV}

\section{Important outcomes}

Failure of treatment as indicated by the need for treatment escalation to NIV, NIPPV, or invasive ventilation

Five studies reported failure of treatment as indicated by escalation to intubation or re-intubation for mechanical ventilation (Frat 2015; Hernandez 2016a; Jing 2019; Shebl 2018; Stephan 2015). We found no evidence of a difference in treatment failure as indicated by escalation to alternative types of oxygen therapy with HFNC use (RR $0.98,95 \% \mathrm{Cl} 0.78$ to 1.22 ; 5 studies, 1758 participants; $\mathrm{I}^{2}=25 \%$; lowcertainty evidence; Analysis 2.1).

We used GRADE to downgrade the certainty of the evidence by two levels to low. We downgraded by one level for inconsistency because we noted some variation in the results which we could not explain, and by one level for study limitations because we judged one study to have a high risk of bias owing to the use of alternative treatment between intermittent HFNC use (see Summary of findings 2).

\section{In-hospital mortality}

Five studies reported mortality up to 90 days (Frat 2015; Hernandez 2016a; Jing 2019; Shebl 2018; Stephan 2015). As well as data specified as in-hospital, we included data in analysis in which time points were up to ICU discharge (Frat 2015), and up to 28 days (Jing 2019). We found no evidence of a difference in mortality according to the type of respiratory support used (RR $0.92,95 \% \mathrm{Cl} 0.64$ to 1.31 ; 5 studies, 1758 participants; $\left.\right|^{2}=44 \%$; low-certainty evidence; Analysis 2.2).

We used GRADE to downgrade the certainty of the evidence by two levels to low. We downgraded by one level for inconsistency because we noted variation in the results of individual studies, and by one level for study limitations because we judged one study to have a high risk of bias owing to the use of alternative treatment between intermittent HFNC use (see Summary of findings 2).

\section{Adverse events}

\section{Respiratory-acquired infections}

Three studies reported data for nosocomial pneumonia (Frat 2015; Hernandez 2016a; Stephan 2015). We found no evidence of a difference in incidence of pneumonia according to the type of respiratory support (RR $0.51,95 \% \mathrm{Cl} 0.17$ to $1.52 ; 3$ studies, 1750 participants; $\left.\right|^{2}=89 \%$; very low-certainty evidence; Analysis 2.3). We used GRADE to downgrade the certainty of the evidence by three levels to very low. We downgraded by two levels for inconsistency because we noted variation in the results of individual studies and a substantial level of statistical heterogeneity, and by one level for study limitations because we judged one study to have a high risk of bias owing to the use of alternative treatment between intermittent HFNC use (see Summary of findings 2).

\section{Nasal mucosa or skin trauma}

No studies measured or reported data for this outcome.

\section{Barotrauma}

One study reported data for barotrauma (Stephan 2015). We used the calculator in RevMan Web 2019 to calculate an effect estimate, and we found no evidence of difference in incidence of pneumothorax according to the type of respiratory support used (RR 1.15, 95\% Cl 0.42 to 3.14; 1 study, 830 participants; low-certainty evidence; Table 5). We used GRADE to downgrade the certainty of the evidence by two levels for imprecision because only one study contributed evidence to this outcome and we noted a wide confidence interval in the effect (see Summary of findings 2).

\section{Length of ICU stay}

Four studies reported length of stay in the ICU (Frat 2015; Hernandez 2016a; Jing 2019; Stephan 2015). We combined data in Frat 2015 and Jing 2019 and found no evidence of a difference in length of stay in the ICU according to the type of respiratory support used (MD -0.72 days, $95 \% \mathrm{Cl}-2.85$ to $1.42 ; 2$ studies, 246 participants; $1^{2}=0 \%$; low-certainty evidence; Analysis 2.5 ). Frat 2015 reported length of stay calculated at 90 days separately for both survivors and non-survivors; in meta-analysis, we included 
only the data for survivors because this included data for a larger number of participants but we note that data for non-survivors indicated a longer length of ICU stay. In the remaining studies in which study authors reported data as median values, there was no evidence of a difference in length of stay in the ICU according to type of respiratory support (see Table 6). From visual inspection, we noted that these data were likely to be right-skewed due to the comparable magnitudes of the mean and standard deviation. This is expected for outcomes such as length of ICU stay due to most participants being discharged in a short time period with some outliers staying significantly longer. However, right skew introduces artefact into calculation of the effect estimate, limiting the interpretation of the result.

We used GRADE to downgrade the certainty of the evidence by two levels to low certainty. We downgraded by one level for inconsistency because we noted a wide variation in length of stay within studies, and by one level for study limitations because we judged one study to have a high risk of bias owing to the use of alternative treatment between intermittent HFNC use (see Summary of findings 2).

\section{Short-term oxygenation $\left(\mathrm{PaO}_{2} / \mathrm{FiO}_{2}\right)$}

Four studies reported this outcome up to 24 hours (Frat 2015; Hernandez 2016a; Jing 2019; Stephan 2015). We did not include data for Hernandez 2016a because these data were reported only for those who were re-intubated or had post-extubation respiratory failure. We found that the ratio of $\mathrm{PaO}_{2} / \mathrm{FiO}_{2}$ was lower when HFNC was used (MD $-58.10 \mathrm{mmHg}, 95 \% \mathrm{Cl}-71.68$ to $-44.51 ; 3$ studies, 1086 participants; $\left.\right|^{2}=8 \%$; low-certainty evidence; Analysis 2.4).

We used GRADE to downgrade the certainty of the evidence by two levels to low. We downgraded by one level for inconsistency because one study had a particularly wide $\mathrm{Cl}$ and we noted differences in $\mathrm{PaO}_{2} / \mathrm{FiO}_{2}$ between studies which could be explained by the different reasons for needing respiratory support between studies. We also downgraded by one level for study limitations because we judged one study to have a high risk of bias owing to the use of alternative treatment between intermittent HFNC use (see Summary of findings 2).

\section{Comfort}

Five studies reported short-term comfort (Chanques 2013; Cong 2019; Frat 2015; Jing 2019; Stephan 2015). We did not combine most data because it was not reported in a comparable manner in each study.

We did not include the data in Chanques 2013, because this was reported in a figure which we could not clearly interpret. In Cong 2019, participants responded to a questionnaire and we used the calculator in RevMan Web 2019 to calculate an effect estimate. We found that more people experienced comfort when HFNC was used (RR 1.30, 95\% Cl 1.10 to 1.53 ; 1 study, 168 participants; Table 5). In Stephan 2015, participants rated their comfort on a five-point scale, which was categorised as 'poor', 'acceptable', or 'good'. Study authors reported no statistically significant differences between groups at one hour $(P=0.32)$ (see Table 6).

We combined data in two studies in which scales were used to rate comfort (Frat 2015; Jing 2019). Jing 2019 used a 10 point scale, and we standardised the $100 \mathrm{~mm}$ visual analogue scale in Frat 2015 so that the scales were comparable; in both scales, higher scores indicate improved comfort. We found that comfort may be improved when HFNC was used (MD $1.33,95 \% \mathrm{Cl} 0.74$ to $1.92 ; 2$ studies, 258 participants; very low-certainty evidence; Analysis 2.6).

We used GRADE to downgrade the certainty of the evidence by three levels to very low. We downgraded by two levels for inconsistency because we noted some variation between study results, and by one level for study limitations because we judged one study to have a high risk of bias owing to the use of alternative treatment between intermittent HFNC use (see Summary of findings 2).

One study also reported data for comfort at day 3 (Stephan 2015). In this study, participants rated their comfort on a five-point scale, which was categorised as 'poor', 'acceptable', or 'good'. Study authors reported no statistically significant differences between groups at day $3(P>0.99)$ (see Table 6$)$. We used GRADE to downgrade the certainty of the evidence by three levels to very low. We downgraded by two levels for imprecision because only one study contributed evidence for this outcome, and one level for study limitations because we noted a high rate of attrition for comfort scores measured at day 3 (see Summary of findings 2).

\section{Additional outcomes}

We found no evidence of a difference in most additional outcomes (duration of respiratory support; long-term $\mathrm{PaO}_{2} / \mathrm{FiO}_{2}$; short- and long-term $\mathrm{PaO}_{2}, \mathrm{SpO}_{2}, \mathrm{PaCO}_{2}$, and long-term respiratory rate; adverse events (ventilator-acquired tracheobronchitis); length of hospital stay; and participant-reported outcomes (dyspnoea)). We noted an effect on the following outcomes:

- Long-term $\mathrm{PaO}_{2} / \mathrm{FiO}_{2}$ : we found that the ratio of $\mathrm{PaO}_{2} / \mathrm{FiO}_{2}$ was lower at more than 24 hours when HFNC was used (MD -31.67 $\mathrm{mmHg}, 95 \% \mathrm{Cl}-49.37$ to $-13.97 ; 2$ studies, 344 participants; $\mathrm{I}^{2}=$ $0 \%$; Analysis 2.8).

- Short-term respiratory rate: we found a slightly improved respiratory rate in the control group (MD -1.06 breaths/min, 95\% $\mathrm{Cl}-1.80$ to -0.32 ; 4 studies, 1090 participants; $\left.\right|^{2}=0 \%$; Analysis 2.11).

We reported the summary effects of additional outcomes in Table 7. No studies reported useable outcome data for atelectasis, PEEP, $\mathrm{SaO}_{2}$, work of breathing, abdominal distension, other participantreported outcomes (dry mouth and refusal to continue treatment), and cost comparison.

\section{Subgroup analysis}

We performed subgroup analysis for treatment failure according to whether participants were treated post-extubation following invasive mechanical ventilation, or whether therapy had not previously included mechanical ventilation.

Although the $P$ value in this subgroup analysis indicated a possible difference between subgroups $(P=0.05)$, subgroups included evidence from few studies (three studies treated participants postextubation, two studies in which participants had not previously used mechanical ventilation) (Analysis 2.1). This limited any meaningful interpretation in this subgroup analysis.

\section{Sensitivity analysis}

We performed sensitivity analyses on 'important outcomes', and presented a summary of these analyses in Table 8 . Whilst most 
sensitivity analyses did not alter our interpretation of the effects, we noted a difference in effect for the following:

- Short-term oxygenation $\left(\mathrm{PaO}_{2} / \mathrm{FiO}_{2}\right)$ : when excluding studies at high or unclear risk of selection bias, we noted the effect estimate indicated no evidence of a difference between types of respiratory support used (MD $-9.30 \mathrm{mmHg}, 95 \% \mathrm{Cl}-80.37$ to $61.77 ; 1$ study, 40 participants).

\section{DISCUSSION}

\section{Summary of main results}

We included 31 studies with 5136 participants; 22 studies were parallel-group design studies and nine were cross-over designs.

In addition, we found 51 ongoing studies (with an estimated recruitment of 12,807 participants). Nineteen studies are awaiting classification because we could not ascertain information on study eligibility (most of these studies were published only as abstracts).

All participants required respiratory support in the ICU. In 18 studies, the intervention was initiated after extubation. In the remaining studies, participants were not previously mechanically ventilated. Twenty-one studies compared HFNC with standard oxygen therapy, and 13 compared HFNC with NIV or NIPPV; three studies included both comparisons. We reported these as two separate comparisons.

\section{HFNC versus standard oxygen therapy}

We found low-certainty evidence that HFNC may lead to less treatment failure as indicated by escalation to alternative types of oxygen therapy. We found moderate-certainty evidence that HFNC probably makes little or no difference to hospital mortality when compared with standard oxygen therapy. HFNC probably results in little or no difference in cases of pneumonia (moderate-certainty evidence), but we were unsure of the effect on nasal mucosa or skin trauma because the certainty of the evidence for this outcome was very low. We found low-certainty evidence of little or no difference in the length of ICU stay according to the type of respiratory support used. For the ratio of partial pressure of arterial oxygen to the fraction of inspired oxygen $\left(\mathrm{PaO}_{2} / \mathrm{FiO}_{2}\right)$, we found no evidence of a difference when measured within 24 hours of treatment but this evidence was very low certainty. Similarly, we were unsure of the evidence for short-term and long-term comfort because this evidence was also of very low certainty.

\section{HFNC versus NIV or NIPPV}

We found low-certainty evidence that HFNC may make little or no difference to treatment failure as indicated by escalation to alternative oxygen therapy or to in-hospital mortality. Although HFNC may result in little or no difference in cases of barotrauma (low-certainty evidence), we were unsure of the effect for pneumonia because of the very low-certainty evidence; no studies reported data for nasal mucosa or skin trauma. HFNC may make little or no difference to the length of ICU stay (low-certainty evidence), and the ratio of $\mathrm{PaO}_{2} / \mathrm{FiO}_{2}$ up to 24 hours may be lower with HFNC use (low-certainty evidence). We were also unsure of the effect of HFNC on short- and long-term comfort because the evidence was of very low certainty.

\section{Overall completeness and applicability of evidence}

We identified 31 studies in this update, which was an additional 20 studies than was included in the previous version of this review (Corley 2017). All studies recruited participants who required respiratory support whilst in the ICU, and therefore provided evidence that was applicable to the population of interest for the review question. These studies could be divided into two indications for respiratory support - those who were given the intervention as they were weaned from invasive mechanical ventilation, and those who had not previously been receiving invasive mechanical ventilation. Whilst the number of studies in the review allowed some subgroup analyses of treatment failure, according to the indication for respiratory support, we still found large variation in the effects at subgroup level between studies in which the respiratory support interventions were started after weaning from mechanical ventilation. We expect that this variation between studies was caused by differences in the illness severity of participants in the included studies.

Although some studies included patient populations with specific underlying conditions, such as COPD or following cardiac surgery, or with specific comorbidities, such as $\mathrm{BMI} \geq 30 \mathrm{~kg} / \mathrm{m}^{2}$ or individuals who were immunocompromised, most included a heterogeneous population with many different causes of respiratory failure in the ICU. This makes it difficult to identify underlying conditions or comorbidities that may respond differently to treatment. Further, the population captured by the studies did not always exclude, but may to an extent under-represent, specific population groups, so our findings may not be generalizable to patients in these groups. Specific examples include pregnant women, participants admitted with exacerbations of asthma, participants with acute pulmonary oedema, participants with tracheostomies, participants with 'Do Not Attempt Cardiopulmonary Resuscitation' (DNACPR) orders, participants with facial abnormalities or trauma, and participants who were haemodynamically unstable or required vasopressors.

Because studies did not all report outcomes of interest for this review, we found that the evidence was still not complete for some outcomes. In particular, few studies reported adverse events that we categorized as respiratory infections, abdominal distension, barotrauma, and nasal mucosa or skin trauma. In addition, we had limited data for many of the patient-relevant outcomes, which limits the conclusions that can be safely drawn (Afshari 2017).

\section{Quality of the evidence}

We used GRADE to downgrade the certainty of the evidence for important review outcomes. We noted inconsistencies in the results for some outcomes, either through visual inspection of the data or from statistical heterogeneity confirmed by the $1^{2}$ statistic. We expected that this inconsistency was caused by variation in the illness severity of participants in each group, and because we were less confident in the effect estimates for these outcomes, we downgraded for this reason. We also downgraded for study limitations owing to some of our assessment of risk of bias; we found that excluding studies that had not adequately reported methods of allocation concealment in the selection process altered the effect in our analysis of treatment failure. We had fewer studies in the analysis of our second comparison (HFNC versus NIV or NIPPV), and we were concerned by the potential bias in one of these studies caused by differences in treatment between each group. Therefore, we also downgraded for study limitations in the relevant 
outcomes in this comparison. A final reason for us to downgrade the certainty of our evidence was because of imprecision; this was particularly relevant for those outcomes for which few studies contributed evidence.

\section{Potential biases in the review process}

We conducted a thorough search in the update and used two review authors to assess eligibility, extract data, and assess risk of bias in included studies; therefore, we reduced potential bias in the review process. We acknowledge that our judgements in the 'Risk of bias' assessments were subjective. It is not possible to blind personnel to the type of respiratory therapy. Although we expected that personnel would aim for optimal respiratory support regardless of the device, we judged that risk of performance bias was unclear in this review. We did not downgrade the certainty of the evidence in this review because of performance bias.

In updating the review, we made minor changes to the methods. The most important change involved the decision to separate the data into two distinct comparison groups (HFNC versus standard oxygen therapy; and HFNC versus NIV or NIPPV). We believed that this was an improvement in the reporting of the results and allowed for greater clarity to the clinical reader. We chose to exclude quasi-randomized studies; these were originally included in the protocol assuming that few studies were likely to be conducted in this field. We noted that adverse events were inconsistently defined in the included studies, and we, therefore, collected data on specific outcomes which we believed to be most relevant to the interventions of interest for the review. Accordingly, we included data only for respiratory infections, abdominal distension, nasal mucosa or skin trauma, and barotrauma (in the comparison group with NIV or NIPPV). Data for adverse events that were previously reported (septic shock, cardiac dysrhythmia, cardiorespiratory arrest, oxygen desaturation, visits to GP), but not reported in this update, are available in Corley 2017. We did not expect these changes to introduce bias into this review update, or to impact the review findings.

We updated this review during the Covid-19 pandemic because the review provides relevant evidence for respiratory support in adults in the ICU. To reduce time to publication, we did not seek additional information from most study authors (for example, we did not seek numerical data to clarify outcomes reported as figures). In addition, we did not attempt contact with authors for studies published as abstracts or studies that were reported as completed in a clinical trial register, and we did not seek translation of studies reported in languages other than English. Therefore, this review has 19 studies awaiting classification, and inclusion of these studies may increase certainty or alter the direction of the effect for some outcomes.

We included one study that was published by two of the review authors of the previous version of this review (Corley 2017) (AC and $\mathrm{JF}$ ); these authors were not involved in this update to the review.

\section{Agreements and disagreements with other studies or reviews}

As HFNC are a relatively novel therapeutic technology, there has been a paucity of data regarding their efficacy for several years. Despite this, review authors of an early systematic review on adults (Kernick 2010), and subsequent systematic reviews (Corley 2017), found preliminary evidence to support the use of HFNC for adults in ICUs requiring respiratory support. Significant efforts to generate high-quality evidence about the short- and long-term efficacy of HFNC in the ICU in recent years are clearly demonstrated by the marked increase in the number of included and ongoing studies within this review. Several literature reviews and meta-analyses have scrutinised this ever-growing body of evidence in a variety of clinical contexts, however, the conclusion drawn by these reviews still shows substantial heterogeneity.

Most reviews found a moderate benefit of HFNC over standard oxygen therapy with regard to treatment failure (Huang 2018; Ni 2018; Rochwerg 2019; Wang 2020; Zhao 2017; Zhu 2016). However, some reviews found no evidence of benefit (Maitra 2016; MonroSomerville 2017; Nedel 2017); these reviews had earlier search dates to our review, included fewer studies and therefore provided less up-to-date evidence. In contrast to our findings, one review investigated treatment failure post-extubation and without prior mechanical respiratory support and found HFNC decreased rates of treatment failure in both subgroups (Xu 2018); this review included patients in settings other than an ICU, such as an Emergency Department. Similar to this review update, all of these reviews found no evidence of a difference in mortality rate. Adverse event outcome data were infrequently reported and rarely subject to statistical analyses, so it was difficult to compare to our review (Rochwerg 2019; Zhu 2016).

Reviews that compared HFNC to NIV or NIPPV produced a more mixed picture for treatment failure. Some reviews found no evidence of difference (Huang 2018; Maitra 2016; Nedel 2017; Wang 2020; Zhao 2017), whilst others showed some benefit of HFNC (Ni 2018); again, variation in the findings of these reviews compared to this update may reflect the earlier search dates in these other systematic reviews. All of these studies found no evidence of a difference in mortality rate. Adverse event data were not reported in any of these studies.

The previous version of this review (Corley 2017) was unable to demonstrate whether HFNC was a safer or more efficient oxygen delivery device compared to other oxygenation devices in the ICU. The paucity of data meant that meta-analysis could only be performed for a few outcomes. We included 20 more studies in this review, providing data for an additional 3164 participants. These studies were more likely to report our outcomes of interest, allowing us to include more participants in each analysis, increasing confidence in our findings. More studies reported on HFNC in comparison to NIV/NIPPV, allowing us to create separate comparison groups comparing HFNC to standard oxygen therapy and NIV/NIPPV respectively. Whilst this review was able to suggest some differences between HFNC and other oxygenation devices, the certainty for most of the outcomes is low or very low, limiting our evaluation of its effectiveness.

\section{AUTHORS' CONCLUSIONS}

\section{Implications for practice}

We evaluated the evidence for HFNC for respiratory support for adults in the intensive care unit. We found low-certainty evidence that HFNC may lead to less treatment failure when compared to standard oxygen therapy. When compared to NIV or NIPPV, we found no evidence of a difference in treatment failure according to the type of respiratory support used, and this evidence was also low-certainty. For most other outcomes, we found no evidence of 
a difference in the effect of using either HFNC or standard oxygen therapy, NIV, or NIPPV. The ratio of $\mathrm{PaO}_{2} / \mathrm{FiO}_{2}$ may be lower with HFNC up to 24 hours after initiation of therapy when compared with NIV or NIPPV but the certainty of this evidence is low.

The evidence in this review is generalizable to adult participants in the intensive care unit who have respiratory failure due to a range of causes. It includes participants who have been weaned from mechanical ventilation as well as participants who have not previously been supported by mechanical ventilation. However, there are some participant groups which were excluded or underrepresented in our population, to which our results may be less generalizable. Examples of these are pregnant women, participants with asthma exacerbation, participants with tracheostomies, participants with 'do not attempt resuscitation' (DNACPR) orders and participants with facial deformities or trauma.

This review did not include 19 studies that are awaiting classification. We found a large number of studies ( 51 studies) that are ongoing and expect that the certainty of the evidence will be improved once these are published. It is also possible that these may alter the interpretation of the effect and, therefore, alter future conclusions in this review.

\section{Implications for research}

We are encouraged by identifying 51 ongoing studies in this field. We anticipate that these studies will provide important evidence for future updates of this review. They may provide further evidence to support meaningful subgroup analyses according to whether or not participants are being weaned from mechanical ventilation, or they may provide sufficient evidence to consider subgroup analysis according to the reason for respiratory failure. We note that two ongoing studies recruited participants with COVID-19; we anticipate that more studies will registered to evaluate the effectiveness of HFNC in this population. To increase the certainty of the evidence, upcoming trials must be of sufficient size and must be methodologically rigorous; they should place particular emphasis on determining the role of HFNC in respiratory failure of different aetiologies and on comparing use of HFNC versus other forms of respiratory support such as non-invasive ventilation, for which we found less evidence. We found few studies reporting participant-relevant outcomes, such as comfort and dyspnoea, as well as limited data for adverse events such as nasal mucosa or skin trauma. We would encourage future studies to consider the effect of each type of respiratory support for these important outcomes.

Whilst we included duration of respiratory support, in the next review update, we propose to alternatively collect data for the number of respiratory support-free days, which would account for loss of participants due to increased anticipated mortality in this population.

\section{ACK NOWLEDGEM NTS}

We would like to thank Jasmin Arrich (Content Editor); Nathan Pace (Statistical Editor); Kevin Dysart and Ravi Tiruvoipati (Peer Reviewers); Stella O'Brien (Consumer Referee); Janne Vendt (Information Specialist); Liz Bickerdike (Network Associate Editor, Acute and Emergency Care Network); Teo Quay and Vernon Hedge (Managing Editors); and Harald Herkner (Co-ordinating Editor) for their help and editorial advice during the preparation of the present update of this systematic review.

We would like to thank the previous review authors (Amanda Corley, Claire M Rickard, Leanne M Aitken, Amy Johnston, Adrian Barnett, and John F Fraser) for their contribution to the protocol and the earlier version of this review (Corley 2017).

We would like to thank Harald Herkner (Content Editor); Cathal Walsh (Statistical Editor); Georgina Imberger, Kevin Dysart, Ravi Tiruvoipati, and Samir Jaber (Peer Reviewers), and Shunjie Chua (Consumer Referee) for help and editorial advice provided during preparation of the earlier version of this review (Corley 2017).

We would also like to thank Karen Hovhannisyan (Former Trials Search Co-ordinator for the Cochrane Anaesthesia, Critical and Emergency Care (ACE) Group) for developing the search strategy, Jane Cracknell (Managing Editor, ACE) for assisting with protocol and review development; and Joan Webster for providing additional advice on the analytical approach for the earlier version of this review (Corley 2017). 


\section{R E F E E N CE S}

\section{References to studies included in this review}

Azoulay 2018 \{published data only\}

* Azoulay E, Lemiale V, Mokart D, Nseir S, Argaud L, Pène F, et al. Effect of high-flow nasal oxygen vs standard oxygen on 28-day mortality in immunocompromised patients with acute respiratory failure. JAMA 2018;320(20):2099-107. [PMID: 30357270]

\section{Brainard 2017 \{published data only\}}

* Brainard J, Scott BK, Sullivan BL, Fernandez-Bustamante A, Piccoli JR, Gebbink MG, et al. Heated humidified high-flow nasal cannula oxygen after thoracic surgery - a randomized prospective clinical pilot trial. Journal of Critical Care 2017;40:225-8. [PMID: 28454060]

\section{Chanques 2013 \{published and unpublished data\}}

Chanques G, Riboulet F, Molinari N, Carr J, Jung B, Prades A, et al. Comparison of three high flow oxygen therapy delivery devices: a clinical physiological cross-over study. Minerva Anestesiologica 2013;79(12):1344-55. [PMID: 23857440]

\section{Cong 2019 \{published data only\}}

Cong L, Zhou LN, Liu HN, Wang JR. Outcomes of high-flow nasal cannula versus non-invasive positive pressure ventilation for patients with acute exacerbations of chronic obstructive pulmonary disease. International Journal of Clinical and Experimental Medicine 2019;12(8):10863-7.

\section{Corley 2014 \{published data only\}10.1007/s00134-015-3765-6}

Corley A, Bull T, Spooner AJ, Barnett AG, Fraser JF. Direct extubation onto high-flow nasal cannulae post-cardiac surgery versus standard treatment in patients with a $\mathrm{BMI} \geq$ 30: a randomised controlled trial. Intensive Care Medicine 2015;41(5):887-94. [PMID: 25851385]

\section{Cuquemelle 2012 \{published and unpublished data\}}

Cuquemelle E, Pham T, Papon JF, Louis B, Danin PE, Brochard L. Heated and humidified high-flow oxygen therapy reduces discomfort during hypoxaemic respiratory failure. Respiratory Care 2012;57(10):1571-7. [PMID: 22417569]

\section{Fernandez 2017 \{published and unpublished data}

Fernandez R, Subira C, Frutos F, Rialp G, Laborda C, Masclans JR, et al. High-flow oxygen therapy for extubation failure prevention in high-risk critically ill patients: a randomized multicenter trial. Intensive Care Medicine Experimental 2015;3(Suppl 1):A164.

* Fernandez R, Subira C, Frutos-Vivar F, Rialp G, Laborda C, Masclans JR, et al. High-flow nasal cannula to prevent postextubation respiratory failure in high-risk non-hypercapnic patients: a randomized multicenter trial. Annals of Intensive Care 2017;7(1):47. [PMID: 28466461]

NCT01820507. Extubation failure prevention in high risk patients by high-flow conditioned oxygen therapy vs. standard oxygen therapy [Prevention of post-extubation respiratory failure in high risk patients by high-flow conditioned oxygen therapy versus standard oxygen therapy]. clinicaltrials.gov/ct2/ show/NCT01820507 (first received 28 March 2013).

\section{Frat 2015 \{published data only\}}

Frat JP, Thille AW, Mercat A, Girault C, Ragot S, Perbet S, et al. High-flow oxygen through nasal cannula in acute hypoxemic respiratory failure. New England Journal of Medicine 2015;372(23):2185-96. [PMID: 25981908]

\section{Futier 2016 \{published data only\}}

Futier E, Paugam-Burtz C, Godet T, Khoy-Ear L, Rozencwajg S, Delay JM, et al. Effect of early postextubation high-flow nasal cannula vs conventional oxygen therapy on hypoxaemia in patients after major abdominal surgery: a French multicentre randomised controlled trial (OPERA). Intensive Care Medicine 2016;42(12):1888-98. [PMID: 27771739]

\section{Grieco 2020 \{published data only\}}

Grieco DL, Menga LS, Raggi V, Bongiovanni F, Anzellotti GM, Tanzarella ES, et al. Physiological comparison of high-flow nasal cannula and helmet noninvasive ventilation in acute hypoxemic respiratory failure. American Journal of Respiratory and Critical Care Medicine 2020;201(3):303-12. [PMID: 31687831]

\section{Hernandez 2016a \{published data only\}}

* Hernandez G, Vaquero C, Colinas L, Cuena R, Gonzalez P, Canabal $A$, et al. Effect of postextubation high-flow nasal cannula vs noninvasive ventilation on reintubation and postextubation respiratory failure in high-risk patients: a randomized clinical trial. JAMA 2016;316(15):1565-74. [PMID: 27706464]

Hernandez G, Vaquero C, Gonzalez P, Colinas L, Garcia S, Canabal A, et al. High flow conditioned oxygen therapy for prevention of reintubation in critically ill patients at high risk for extubation failure: a multicenter randomised controlled trial. Intensive Care Medicine Experimental 2015;3(Suppl 1):A823.

NCT01191489. High-flow conditioned oxygen therapy versus non-invasive ventilation: prevention of post-extubation failure [Comparison between high-flow conditioned oxygen therapy and non-invasive ventilation in prevention of postextubation respiratory failure. A randomized controlled trial]. clinicaltrials.gov/ct2/show/study/NCT01191489 (first received 30 August 2010).

\section{Hernandez 2016b \{published data only\}}

* Hernandez G, Vaquero C, Gonzalez P, Subira C, FrutosVivar F, Rialp G, et al. Effect of postextubation high-flow nasal cannula vs conventional oxygen therapy on reintubation in low-risk patients: a randomized clinical trial. JAMA 2016;315(13):1354-61. [PMID: 26975498]

NCT01191489. High-flow conditioned oxygen therapy versus non-invasive ventilation: prevention of post-extubation failure [Comparison between high-flow conditioned oxygen therapy and non-invasive ventilation in prevention of postextubation respiratory failure. A randomized controlled trial]. clinicaltrials.gov/ct2/show/study/NCT01191489 (first received 30 August 2010). 
Hu 2020 \{published data only\}

Hu TY, Lee CH, Cheng KH, Tan MC, Hua HF, Kuo LK. Effect of high-flow nasal oxygen vs. conventional oxygen therapy on extubation outcomes and physiologic changes for patients with high risk of extubation failure in the medical ICU: a tertiary center, randomized, controlled trial. International Journal of Gerontology 2020;14(1):36-41. [EMBASE: 2005059557]

\section{Jing 2019 \{published data only\}}

Jing G, Li J, Hao D, Wang T, Sun Y, Tian H, et al. Comparison of high flow nasal cannula with noninvasive ventilation in chronic obstructive pulmonary disease patients with hypercapnia in preventing postextubation respiratory failure: a pilot randomized controlled trial. Research in Nursing \& Health 2019;42(3):217-25. [PMID: 30887549]

\section{Lee 2018 \{published data only\}}

* Lee DH, Kim EY, Seo GJ, Suh HJ, Huh JW, Hong SB, et al. Global and regional ventilation during high flow nasal cannula in patients with hypoxia. Acute \& Critical Care 2018;33(1):7-15. [PMID: 31723854]

Lee DH, Lim CM, Koh Y, Hong SB, WonHuh J, Seo GJ, et al. Global and regional ventilation during highflow nasal cannula in patients with hypoxia. Critical Care Medicine 2016;44(12):325.

\section{Lemiale 2015 \{published data only\}}

Lemiale V, Mokart D, Mayaux J, Lambert J, Rabbat A, Demoule A, et al. The effects of a 2-h trial of high-flow oxygen by nasal cannula versus Venturi mask in immunocompromised patients with hypoxemic acute respiratory failure: a multicenter randomized trial. Critical Care 2015;19:380. [PMID: 26521922]

\section{Longhini 2019 \{published data only\}}

Longhini F, Pisani L, Lungu R, Comellini V, Fruni A, Garofalo E, et al. High-flow oxygen therapy after noninvasive ventilation interruption in patients recovering from hypercapnic acute respiratory failure: a physiological crossover trial. Critical Care Medicine 2019;47(6):E506-11.

\section{Maggiore 2014 \{unpublished data only\}}

Antonicelli F, Cataldo A, Festa F, Idone R, Moccaldo A, Antonelli $\mathrm{M}$, et al. High-flow oxygen therapy through nasal cannulae versus low-flow oxygen therapy via venturi mask after extubation in adult, critically ill patients. In: 31st International Symposium on Intensive Care and Emergency Medicine; 2011 March; Brussels, Belgium. Brussels, Belgium, 2011.

Idone FA, Festa R, Antonicelli F, Di Muzio F, Maniglia D, Cataldo A, et al. Nasal high-flow oxygen therapy vs. standard oxygen therapy via venturi mask after extubation: preliminary results of a randomized, controlled trial. In: 23rd Annual Congress of the European Society of Intensive Care Medicine (ESICM); 2010 October; Barcelona, Spain. 2010.

\footnotetext{
* Maggiore SM, Idone FA, Vaschetto R, Festa R, Cataldo A, Antonicelli $F$, et al. Nasal high-flow versus Venturi mask oxygen therapy after extubation. Effects on oxygenation, comfort, and clinical outcome. American Journal of Respiratory and Critical Care Medicine 2014;190(3):282-8. [PMID: 25003980]
}

Moccaldo A, Vaschetto R, Bernini V, Antonicelli F, Festa R, Idone $F$, et al. Oxygen after extubation: comparison between high flow system (Optiflow) and Venturi mask [Ossigenoterapia dopo estubazione: confronto tra sistema ad alti flussi (Optiflow) e maschera venturi]. Minerva Anestesiology 2011;77(10 Suppl 2):169.

\section{Mauri 2017a \{published data only\}}

Mauri T, Alban L, Turrini C, Cambiaghi B, Carlesso E, Taccone P, et al. Optimum support by high-flow nasal cannula in acute hypoxemic respiratory failure: effects of increasing flow rates. Intensive Care Medicine 2017;43(10):1453-63. [PMID: 28762180]

\section{Mauri 2017b \{published data only\}}

* Mauri T, Turrini C, Eronia N, Grasselli G, Volta CA, Bellani G, et al. Physiologic effects of high-flow nasal cannula in acute hypoxemic respiratory failure. American Journal of Respiratory and Critical Care Medicine 2017;195(9):1207-15. [PMID: 27997805]

\section{Parke 2011 \{published and unpublished data\}}

Parke RL, McGuinness SP, Eccleston ML. A preliminary randomised controlled trial to assess effectiveness of nasal high-flow oxygen in intensive care patients. Respiratory Care 2011;56(3):265-70. [PMID: 21255498]

\section{Parke 2013a \{published and unpublished data\}}

Parke R, McGuinness S, Dixon R, Jull A. Open-label, phase II study of routine high-flow nasal oxygen therapy in cardiac surgical patients. British Journal of Anaesthesia 2013;111(6):925-31. [PMID: 23921199]

\section{Rittayamai 2014 \{published and unpublished data\}}

Rittayamai N, Tscheikuna J, Rujiwit P. High-flow nasal cannula versus conventional oxygen therapy after endotracheal extubation: a randomized crossover physiologic study. Respiratory Care 2014;59(4):485-90. [PMID: 24046462]

\section{Schwabbauer 2014 \{published data only\}}

Riessen R, Schwabbauer N, Berg B, Blumenstock G, Haap M, Hetzel J. Nasal high-flow oxygen in patients with hypoxic respiratory failure: effect of functional and subjective respiratory parameters compared to conventional oxygen therapy and noninvasive ventilation. In: 32nd International Symposium on Intensive Care and Emergency Medicine; 2012 March; Brussels, Belgium. 2012.

\section{* Schwabbauer N, Berg B, Blumenstock G, Haap M, Hetzel J, Riessen R. Nasal high-flow oxygen therapy in patients with hypoxic respiratory failure: effect on functional and subjective respiratory parameters compared to conventional oxygen therapy and non-invasive ventilation (NIV). BMC Anesthesiology 2014;14(66):1-7. [PMID: 25110463]}

\section{Shebl 2018 \{published data only\}}

Shebl E, Embarak S. High-flow nasal oxygen therapy versus noninvasive ventilation in chronic interstitial lung disease patients with acute respiratory failure. Egyptian Journal of Chest Diseases and Tuberculosis 2018;67(3):270-5. [EMBASE: 625864420] 
Song 2017 \{published data only\}

Song HZ, Gu JX, Xiu HQ, Cui W, Zhang GS. The value of highflow nasal cannula oxygen therapy after extubation in patients with acute respiratory failure. Clinics 2017;72(9):562-7. [PMID: 29069260]

\section{Stephan 2015 \{published data only\}}

Stephan F, Barrucand B, Petit P, Rezaiguia-Delclaux S, Medard A, Delannoy B, et al. BiPAP non invasive ventilation compared to Optiflow ${ }^{\circledR}$ system in patients with hypoxemia after cardiothoracic surgery: multicenter, randomized, non-inferiority and open (BiPOP study). Annales Francaises d'Anésthesie et de Réanimation 2014;33:A72.

* Stephan F, Barrucand B, Petit P, Rezaiguia-Delclaux S, Medard A, Delannoy B, et al. High-flow nasal oxygen vs noninvasive positive airway pressure in hypoxemic patients after cardiothoracic surgery: a randomized clinical trial. JAMA 2015;313(23):2331-9. [PMID: 25980660]

Stephan F, Barrucand B, Petit P, Rezaiguia-Delclaux S, Medard A, Delannoy B. Bilevel positive airway pressure versus OPTIFLOW in hypoxemic patients after cardiothoracic surgery (the BiPOP study): a multicenter, randomized, noninferiority, open trial. American Journal of Respiratory and Critical Care Medicine 2014;189:A6572.

\section{Vargas 2015 \{published data only\}}

Vargas F, Saint-Leger M, Boyer A, Bui NH, Hilbert G. Physiologic effects of high-flow nasal cannula oxygen in critical care subjects. Respiratory Care 2015;60(10):1369-76. [PMID: 25944940]

\section{Vourc'h 2020 \{published and unpublished data\}}

Vourc'h M, Nicolet H, Volteau C, Caubert L, Chabbert C, Lepoivre T, et al. High-flow therapy by nasal cannulae versus high-flow face mask in severe hypoxemia after cardiac surgery: a single-center randomized controlled study - the HEART FLOW study. Journal of Cardiothoracic and Vascular Anesthesia 2020;34:157-65. [PMID: 31230964]

\section{Yu 2017 \{published data only\}}

Yu Y, Qian X, Liu C, Zhu C. Effect of high-flow nasal cannula versus conventional oxygen therapy for patients with thoracoscopic lobectomy after extubation. Canadian Respiratory Journal 2017;2017:7894631. [PMID: 28298878]

\section{Zochios 2018 \{published data only\}}

* Zochios V, Collier T, Blaudszun G, Butchart A, Earwaker M, Jones $\mathrm{N}$, et al. The effect of high-flow nasal oxygen on hospital length of stay in cardiac surgical patients at high risk for respiratory complications: a randomised controlled trial. Anaesthesia 2019;73(12):1478-88. [PMID: 30019747]

\section{References to studies excluded from this review}

\section{Coudroy 2019 \{published data only\}}

Coudroy R, Frat JP, Ehrmann S, Pène F, Terzi N, Decavèle M, et al. High-flow nasal oxygen therapy alone or with non-invasive ventilation in immunocompromised patients admitted to ICU for acute hypoxemic respiratory failure: the randomised multicentre controlled FLORALI-IM protocol. BMJ Open 2019;9:e029798. [PMID: 31401603]

Delorme 2017 \{published data only\}

Delorme M, Bouchard PA, Simon M, Simard S, Lellouche F. Effects of high-flow nasal cannula on the work of breathing in patients recovering from acute respiratory failure. Critical Care Medicine 2017;45(12):1981-8. [PMID: 28857852]

Di Mussi 2016 \{published data only\}

Di Mussi R, Spadaro S, Volta CA, Mariani M, Colaprico A, Antonio $C$, et al. Effects of high flow nasal cannula oxygen on diaphragmatic electrical activity in the post extubation period. Intensive Care Medicine Experimental (29th Annual Congress of the European Society of Intensive Care Medicine, ESICM 2016) 2016;4 (Suppl 1):no pagination. [EMBASE: 617991736]

\section{Lemiale 2016 \{published data only\}}

* Lemiale V, Resche-Rigon M, Mokart D, Pene F, Argaud L, Mayaux J, et al. High-flow nasal cannula oxygenation in immunocompromised patients with acute hypoxemic respiratory failure: a Groupe de Recherche Respiratoire en Reanimation Onco-Hematologique study. Critical Care Medicine 2016;20:20. [PMID: 27655324]

Lemiale V, Resche-Rigon M, Mokart D, Pene F, Faucher E, Guitton C, et al. High-flow nasal cannula for acute respiratory failure in immunocompromised patients. Annals of Intensive Care (French Intensive Care Society, International Congress Reanimation) 2016;6:no pagination.

\section{Liu 2019 \{published data only\}}

Liu F, Shao Q, Jiang R, Zeng ZG, Liu Y, Li Y, et al. High-flow oxygen therapy to speed weaning from mechanical ventilation: a prospective randomized study. Americal Journal of Critical Care 2019;29(5):370-6. [PMID: 31474607]

Pennisi 2019 \{published data only\}

Pennisi MA, Bello G, Congedo MT, Montini L, Nachira D, Ferretti GM, et al. Early nasal high-flow versus Venturi mask oxygen therapy after lung resection: a randomized trial. Critical Care 2019;23(1):68. [PMID: 30819227]

\section{Sklar 2018 \{published data only\}}

Sklar MC, Dres M, Rittayamai N, West B, Grieco DL, Telias I, et al. High-flow nasal oxygen versus noninvasive ventilation in adult patients with cystic fibrosis: a randomized crossover physiological study. Annals of Intensive Care 2018;8:85. [PMID: 30187270]

\section{Thille 2018 \{published data only\}}

Thille AW, Muller G, Gacouin A, Coudroy R, Demoule A, Sonneville R, et al. High-flow nasal cannula oxygen therapy alone or with non-invasive ventilation during the weaning period after extubation in ICU: the prospective randomised controlled HIGH-WEAN protocol. BMJ Open 2018;8(9):e023772. [PMID: 30185583]

\section{Thille 2019 \{published data only\}}

Thille AW, Muller G, Gacouin A, Coudroy R, Decavele M, Sonneville R, et al. Effect of postextubation high-flow nasal 
oxygen with noninvasive ventilation vs high-flow nasal oxygen alone on reintubation among patients at high risk of extubation failure: a randomized clinical trial. JAMA 2019;322(15):1465-75. [PMID: 31577036]

\section{References to studies awaiting assessment}

Arman 2017 \{published data only\}

* Arman PD, Varn MN, Povian S, Davis A, Uchakin P, Bhar A, et al. Effects of direct extubation to high-flow nasal cannula compared to standard nasal cannula in patients in the intensive care unit. American Journal of Respiratory and Critical Care Medicine 2017;195:A1887.

\section{Guoqiang 2018 \{published data only\}}

* Guoqiang J, Li J, Hao D, Wang T, Wang X. Comparison of high flow nasal cannula with noninvasive ventilation in facilitating weaning COPD from invasive ventilation: a prospective randomized controlled study. European Respiratory Journal 2018;52(Suppl 62):PA3338.

\section{Gupta 2016 \{published data only\}}

Gupta S, Govil D, Srinivasan S, Patel SJ, N JK, Gupta A, et al. High flow nasal cannula (HFNC) as an alternative to noninvasive ventilation (NIV) in acute respiratory failure (ARF) in immunosuppressed patients - an Indian post liver transplant experience. Intensive Care Medicine Experimental 2016;4(30):A406.

\section{Ischaki 2019 \{published data only\}}

Ischaki E, Pantazopoulos I, Manoulakas E, Boutlas S, Papalampidou A, Moylan M, et al. Nasal high flow versus noninvasive ventilation in patients with acute exacerbation of chronic obstructive pulmonary disease.. European Respiratory Journal 2019;54(Suppl 63):PA2327.

\section{ISRCTN17399068 \{published data only\}}

* ISRCTN17399068. High-flow nasal oxygen (HFNO) in highrisk cardiac surgical patients [A randomised controlled trial of high-flow nasal oxygen (Optiflow ${ }^{\mathrm{TM}}$ ) and standard oxygen therapy in high-risk patients after cardiac surgery]. isrctn.com/ ISRCTN17399068 (first received 15 July 2015).

\section{Lee 2016 \{published data only\}}

Lee MK, Kim SH, Lee WY, Yong SJ, Lee SJ, Jung YR. The efficacy of high-flow nasal cannulae oxygen therapy in severe acute exacerbation of chronic obstructive pulmonary disease: a randomized controlled trial. European Respiratory Journal 2018;48(Suppl 60):PA3058.

\section{Longhini 2017 \{published data only\}}

Longhini F, Pisani L, Lungu R, Comellini V, Cammarota G, Bruni A, et al. High-flow oxygen therapy in hypercapnic patients recovering from an episode of acute on-chronic respiratory failure. European Respiratory Journal. 2017;50:PA1862.

\section{Macari 2019 \{published data only\}}

Macari EA, Bubenheim M, Le Bouar G, Carpentier D, Grangé S, Boyer D, et al. High - f low oxygen therapy vs non invasive ventilation - a prospective cross-over physiological study of alveolar recruitment in acute respiratory failure. Annals of Intensive Care 2019;9(Suppl 1):CO-27.

* Macari EA, Bubenheim M, Le Bouar G, Carpentier D, Grangé S, Boyer $D$, et al. High-flow oxygen therapy vs non invasive ventilation: a prospective randomized cross-over physiological study of alveolar recruitment in acute respiratory failure. European Respiratory Journal 2019;54(Suppl 63):OA4924.

\section{Menga 2019 \{published data only\}}

Menga IS, Raggi V, Bongiovanni F, Grieco DL, Eleuteri D, Bello G, et al. Helmet non-invasive ventilation versus high flow nasal oxygen in acute hypoxemic respiratory failure: physiological effects. Critical Care 2019;23(72):341.

\section{Papachatzakis 2017 \{published data only\}}

Papachatzakis I, Velentza L, Kontogiannis S, Trakada G. High flow nasal cannula with warm humidified air versus noninvasive mechanical ventilation in respiratory failure type II. European Respiratory Journal 2017;50:PA2182.

\section{Perbet 2014 \{published data only\}}

Perbet S, Gerst A, Chabanne R, Soummer A, Faure JS, Pascal J, et al. High-flow nasal oxygen cannula versus conventional oxygen therapy to prevent postextubation lung aeration loss: a multicentric randomized control lung ultrasound study. In: 27th Annual Congress of the European Society of Intensive Care Medicine; 2014 September; Barcelona, Spain. 2014.

\section{Saeed 2015 \{published data only\}}

Saeed A, Wagih K, Huusein N. Evaluation of nasal Optiflow device in management of COPD patients in acute exacerbations. European Respiratory Journal (European Respiratory Society Annual Congress) 2015;46:no pagination. [72105945]

\section{Schreiber 2017 \{published data only\}}

Schreiber AF, Lu Q, Adam N, Langeron O. High-flow nasal cannula versus noninvasive ventilation on lung re-aeration and diaphragm function. European Respiratory Journal 2017;50:PA2180.

\section{Theerawit 2017 \{published data only\}}

NCT02918786. The efficacy of the Whispherflow CPAP system versus high flow nasal cannula in patients at high risk for postextubation failure. clinicaltrials.gov/show/NCT02918786 (first received 29 September 2016).

* Theerawit P, Natpobsuk N, Sutherasan Y. The efficacy of the Whispherflow CPAP system versus high flow nasal cannula in patients at high risk for postextubation failure. Intensive Care Medicine Experimental 2017;5(Suppl 1):no pagination.

\section{Tseng 2019 \{published data only\}}

Tseng CW, Chao KY, Chiang CE, Liu WL, Chou WR, Wu HL, et al. The efficacy of heated humidifier high-flow nasal cannula compared with noninvasive positive-pressure ventilation in prevention of reintubation in patients with prolonged mechanical ventilation. European Respiratory Journal 2019;54:RCT5097. 
Yang 2019 \{published data only\}

Yang S, Zhang G, Liu Z, Yan Q, Meng S, Zhao B, et al. Effect of high-flow nasal cannula oxygen therapy on diaphragmatic function in patients with acute exacerbation of chronic obstructive pulmonary disease: a prospective randomized controlled trial. Chinese Critical Care Emergency Medicine 2019;31(5):551-5. [PMID: 31198138]

\section{Zhang 2018 \{published data only\}}

Zhang J, Wu F, Meng L, Zeng C, Lu Y. Efficacy and safety of sequential nasal high flow oxygen therapy in patients with chronic obstructive pulmonary disease. Chinese Medical Journal 2018;98(2):109-12.

\section{Zhao 2019 \{published data only\}}

* Zhao Y, Li J, Liu C. Application of heated humidified high flow nasal cannula oxygen therapy in elderly patients with advanced lung cancer complicated with type I respiratory failure. Nursing of Integrated Traditional Chinese and Western Medicine 2019;5(12):86-8.

\section{Zhu 2017 \{published data only\}}

Zhu Z, Liu Y, Wang Q, Wang S. Preliminary evaluation of sequential therapy by high flow nasal cannula oxygen therapy following endotracheal tube extubation in mechanically ventilated patients. Chinese Critical Care Medicine 2017;29(9):778-82. [PMID: 28936951]

\section{References to ongoing studies}

\section{ACTRN12617000694314 \{published data only\}}

ACTRN12617000694314. Prophylactic post-operative high flow nasal oxygen therapy versus conventional oxygen therapy in obese patients undergoing bariatric surgery: a randomised controlled pilot study. apps.who.int/trialsearch/Trial2.aspx? TrialID=ACTRN12617000694314 (first received 15 May 2017).

\section{ChiCTR1800014553 \{published data only\}}

ChiCTR1800014553. Comparative study of nasal high flow oxygen therapy and noninvasive positive pressure ventilation for moderate AECOPD: randomized open non inferiority trial. apps.who.int/trialsearch/Trial2.aspx? TrialID=ChiCTR1800014553\%20 (first received 21 January 2018).

ChicTR1800017313 \{published data only\}

ChiCTR1800017313. Clinical application of highflow nasal cannula therapy in patients with posttraumatic ARDS. apps.who.int/trialsearch/Trial2.aspx? TrialID=ChiCTR1800017313 (first received 24 July 2017).

\section{ChiCTR1800018530 \{published data only\}}

ChiCTR1800018530. A multicenter randomized controlled trial for invasive-high flow oxygen therapy and invasivenoninvasive sequential therapy for severe respiratory failure caused by COPD. apps.who.int/trialsearch/Trial2.aspx? TrialID=ChiCTR1800018530 (first received 22 September 2018).

\section{ChiCTR1900020826 \{published data only\}}

ChiCTR1900020826. A multi-center randomized controlled clinical trial for standardized respiratory support treatment for acute severe virus pneumonia. apps.who.int/trialsearch/ Trial2.aspx?TrialID=ChiCTR1900020826 (first received 20 January 2019).

\section{ChiCTR1900021091 \{published data only\}}

ChiCTR1900021091. Improvement of ventilator weaning rate in patients with severe pneumonia by HFNC. apps.who.int/ trialsearch/Trial2.aspx?TrialID=ChiCTR1900021091 (first received 28 January 2019).

ChiCTR1900022241 \{published data only\}ChiCTR1900022241

* ChiCTR1900022241. Optimization of respiratory support of AIDS patients complicated with acute respiratory failure [Efficacy of high-flow nasal cannula oxygen therapy and noninvasive positive pressure ventilation for acute respiratory failure in patients with AIDS: a prospective, randomized, controlled trial]. apps.who.int/trialsearch/Trial2.aspx? TrialID=ChiCTR1900022241\%20 (first received 31 March 2019).

\section{ChiCTR1900023296 \{published data only\}}

* ChiCTR1900023296. Sequential therapy effects of HFNC on patients with thoracic trauma combined with ARDS from weaning: a prospective single-centered randomized controlled study. https://apps.who.int/trialsearch/Trial2.aspx? TrialID=ChiCTR1900023296 (first received 21 May 2019).

\section{ChiCTR1900025974 \{published data only\}}

* ChiCTR1900025974. HFNC vs NPPV after early extubation for patients with AECOPD [High-flow nasal cannula versus non-invasive positive pressure ventilation therapy after early extubation for patients with acute exacerbation of chronic obstructive pulmonary disease: a multicenter randomized controlled trial]. apps.who.int/trialsearch/Trial2.aspx? TrialID=ChiCTR1900025974 (first received 16 September 2019).

\section{ChicTR-INR-17011850 \{published data only\}}

ChiCTR-INR-17011850. Sequential oxygen therapy via high-flow nasal cannula following invasive ventilation in AECOPD induced hypercapnic respiratory failure: a prospective randomized controlled study. apps.who.int/trialsearch/Trial2.aspx? TrialID=ChiCTR-INR-17011850 (first received 3 July 2017).

ChiCTR-INR-17012720 \{published data only\}

* ChiCTR-INR-17012720. Clinical effect of high flow nasal cannula oxygen therapy on sequential treatment of invasive ventilation in patients with acute exacerbation of chronic obstructive pulmonary disease [Application of high flow nasal cannula oxygen therapy in patients with acute exacerbation of chronic obstructive pulmonary disease]. apps.who.int/ trialsearch/Trial2.aspx?TrialID=ChiCTR-INR-17012720 (first received 19 September 2017).

\section{Cortegiani 2019 \{published data only\}}

* Cortegiani A, Longhini F, Carlucci A, Scala R, Groff P, Garofalo $A B$, et al. High-flow nasal therapy versus noninvasive ventilation in COPD patients with mild-to-moderate hypercapnic acute respiratory failure: study protocol for a noninferiority randomized clinical trial. Trials 2019;20(1):450. [PMID: 31331372] 
NCT03370666. High flow nasal therapy versus non-invasive ventilation in COPD exacerbation [High flow nasal therapy versus non-invasive ventilation in mild to moderate acute hypercapnic respiratory failure: a non-inferiority randomized trial]. clinicaltrials.gov/ct2/show/record/NCT03370666 (first received 12 December 2017).

\section{CTRI/2018/09/015717 \{published data only\}}

* CTRI/2018/09/015717. To study the effect of oxygen therapy given at very high rate in patient with sudden onset of breathing failure [High-flow oxygen through nasal cannula in acute hypoxemic respiratory failure]. apps.who.int/trialsearch/ Trial2.aspx?TrialID=CTRI/2018/09/015717 (first received 14 September 2018).

\section{ISRCTN16912075 \{published data only\}}

* ISRCTN16912075. RECOVERY respiratory support: respiratory strategies in patients with coronavirus COVID-19 - CPAP, highflow nasal oxygen, and standard care [In adult patients with known or suspected COVID-19, does the use of continuous positive airway pressure (CPAP) or high-flow nasal oxygen (HFNO), compared with standard care reduce mortality or need for tracheal intubation?]. isrctn.com/ISRCTN16912075 (first received 2 April 2020).

\section{JPRN-jRCTs052180236 \{published data only\}}

* JPRN-jRCTs052180236. Ja-NP-Hi trial [Japanese, multicenter, randomized controlled trial of noninvasive positive pressure ventilation (NPPV) versus high-flow nasal cannula oxygen therapy (HFNC) for severe acute hypoxemic respiratory failure - Ja-NP-Hi trial]. apps.who.int/trialsearch/Trial2.aspx? TrialID=JPRN-jRCTs052180236 (first received 30 March 2019).

JPRN-UMIN000028827. Japanese, multicenter, randomized controlled trial of noninvasive positive pressure ventilation (NPPV) versus high-flow nasal cannula oxygen therapy (HFNC) for acute hypoxemic respiratory failure. apps.who.int/ trialsearch/Trial2.aspx?TrialID=JPRN-UMIN000028827 (first received 25 August 2017).

\section{NCT01166256 \{published data only\}}

NCT01166256. Comparison between high-flow nasal cannula system and non-invasive ventilation in acute hypoxemic respiratory failure [Prospective, open-labeled, randomized controlled trial of comparison between high-flow nasal cannula system and non-invasive ventilation in acute hypoxemic respiratory failure]. clinicaltrials.gov/ct2/show/NCT01166256? term=NCT01166256\&rank=1 (first received 19 July 2010).

\section{NCT01702779 \{published data only\}}

NCT01702779. Nasal humidified high flow oxygen during weaning from mechanical ventilation: ultrasonography study. clinicaltrials.gov/ct2/show/NCT01702779? term=NCT01702779\&rank=1 (first received 24 April 2012).

\section{NCT02107183 \{published data only\}}

NCT02107183. Impact of nasal high-flow vs Venturi mask oxygen therapy on weaning outcome: a multicenter, randomized, controlled trial. clinicaltrials.gov/ct2/show/ NCT02107183?term=NCT02107183\&rank=1 (first received 4 April 2014).

\section{NCT02123940 \{published data only\}}

NCT02123940. Treatment strategy in patients with high-risk of postextubation distress in ICU based on a lung ultrasound score versus standard strategy. clinicaltrials.gov/ct2/show/ NCT02123940?term=NCT02123940\&rank=1 (first received 11 April 2014).

\section{NCT02290548 \{published data only\}}

* NCT02290548. Effect of high-flow nasal oxygen on extubation outcome [Effect of high-flow nasal oxygen vs standard oxygen therapy on extubation outcome with high risk of extubation failure in medical ICU patients]. clinicaltrials.gov/ct2/show/ record/NCT02290548 (first received 14 November 2014).

\section{NCT02464696 \{published data only\}}

* NCT02464696. Early non-invasive ventilation and hematological malignancies [Early non-invasive ventilation in patients with hypoxemic respiratory failure and hematological malignancies: a prospective randomized controlled trial]. clinicaltrials.gov/ct2/show/record/NCT02464696 (first received 8 June 2015).

\section{NCT02713737 \{published data only\}}

* NCT02713737. The correlation between sleep quality and atrial fibrillation undergoing high-flow nasal cannula oxygen (HFNC) [Impact of high-flow nasal cannula oxygen (HFNC) versus non-invasive ventilation associated with sleep quality on atrial fibrillation in hypoxemic patients after coronary surgery]. clinicaltrials.gov/ct2/show/record/NCT02713737 (first received 21 March 2016).

\section{NCT03014869 \{published data only\}}

* NCT03014869. High flow nasal cannula vs NPPV in moderate chronic obstructive pulmonary disease exacerbation [Comparison of high flow nasal cannula and non-invasive positive ventilation (NPPV) in moderate chronic obstructive pulmonary disease exacerbation (AECOPD)]. clinicaltrials.gov/ ct2/show/NCT03014869 (first received 9 January 2017).

\section{NCT03133520 \{published data only\}}

NCT03133520. Effectiveness of high flow oxygen therapy in patients with hematologic malignancy acute hypoxemic respiratory failure. clinicaltrials.gov/ct2/show/NCT03133520 (first received 28 April 2017).

NCT03171935 \{published data only\}

* NCT03171935. Wean early with HFNCO vs NPPV in patients with AHRF [Wean early with high-flow nasal cannula oxygenation versus noninvasive positive pressure ventilation in patients with acute hypoxemic respiratory failure: a multicenter, randomized, controlled trial (the WHEN study)]. clinicaltrials.gov/ct2/show/record/NCT03171935 (first received 31 May 2017)

NCT03229460 \{published data only\}

* NCT03229460. High flow nasal oxygen therapy in perioperative period of the adult with hypercapnic and hypoxemic respiratory failure. clinicaltrials.gov/ct2/show/ record/NCT03229460 (first received 25 July 2017). 


\section{NCT03246893 \{published data only\}}

* NCT03246893. Efficacy of HFNC versus NIV for prevent reintubation in sepsis patients [Efficacy of high flow oxygen nasal cannula versus non-invasive positive pressure ventilation after extubation in sepsis patients]. clinicaltrials.gov/ct2/show/ NCT03246893 (first received 11 August 2017).

\section{NCT03282552 \{published data only\}}

* NCT03282552. High flow oxygen therapy versus conventional oxygen therapy in cardiac surgery patients [High flow oxygen therapy versus conventional oxygen therapy in cardiac surgery patients - OPTICAR study]. clinicaltrials.gov/ct2/show/ NCT03282552 (first received 14 September 2017).

\section{NCT03361683 \{published data only\}}

* NCT03361683. Post-extubation high-flow nasal oxygen for preventing extubation failure [Post-extubation high-flow nasal oxygen vs. conventional oxygen in patients recovered from acute hypoxemic respiratory failure for preventing extubation failure]. clinicaltrials.gov/ct2/show/record/NCT03361683 (first received 5 December 2017).

\section{NCT03430258 \{published data only\}}

* NCT03430258. High-flow nasal cannula oxygen therapy with the chest trauma patients. clinicaltrials.gov/ct2/show/record/ NCT03430258 (first received 12 February 2018).

NCT03488628 \{published data only\}

* NCT03488628. High-flow nasal oxygen therapy for exacerbation of chronic pulmonary obstructive disease [High-flow nasal oxygen therapy for exacerbation of chronic pulmonary obstructive disease: a randomized, open-label, single-center, pilot study.]. clinicaltrials.gov/ct2/show/ NCT03488628 (first received 5 April 2018).

\section{NCT03515031 \{published data only\}}

NCT03515031. High flow nasal cannulae vs venturi mask in respiratory failure due to pneumonia. clinicaltrials.gov/ct2/ show/record/NCT03515031 (first received 3 May 2018).

\section{NCT03607357 \{published data only\}}

* NCT03607357. High flow nasal oxygen and acute left heart failure [The effect of post-extubation high flow nasal oxygen in patients with acute left heart failure: a clinical multi-center study]. clinicaltrials.gov/ct2/show/NCT03607357 (first received 31 July 2018).

\section{NCT03632577 \{published data only\}}

NCT03632577. High flow oxygen versus non-invasive ventilation associated to automated flow oxygen titration after patient extubation [High flow oxygen (HFO) versus non-invasive ventilation (NIV) associated to automated flow oxygen titration (AFOT) after extubation in patient with respiratory risk: noninferiority prospective comparative study]. clinicaltrials.gov/ ct2/show/record/NCT03632577 (first received 15 August 2018).

\section{NCT03643939 \{published data only\}}

* NCT03643939. High-flow nasal oxygen cannula compared to non-invasive ventilation in adult patients with acute respiratory failure [Randomized adaptive trial of high-flow nasal oxygen cannula compared to non-invasive ventilation for acute respiratory failure]. clinicaltrials.gov/ct2/show/record/ NCT03643939 (first received 23 August 2018).

NCT03788304 \{published data only\}

* NCT03788304. High flow nasal cannula versus non-invasive ventilation in prevention of escalation to invasive mechanical ventilation in patients with acute hypoxemic respiratory failure. clinicaltrials.gov/ct2/show/NCT03788304 (first received 27 December 2018).

\section{NCT03811158 \{published data only\}}

* NCT03811158. The edi level and cardiopulmonary function between HHHFNC and unheated humidified high-flow oxygen mask in COPD [The diaphragm activity level and cardiopulmonary function between heated humidified highflow nasal cannula and unheated humidified high-flow oxygen mask in acute exacerbation of COPD patients as post-extubation respiratory support]. clinicaltrials.gov/ct2/show/record/ NCT03811158 (first received 22 January 2019).

NCT03865056 \{published data only\}

* NCT03865056. Therapy with high-flow oxygen by nasal cannula vs noninvasive ventilation in patients with acute hypoxemic respiratory failure: a crossover physiologic study. clinicaltrials.gov/ct2/show/record/NCT03865056 (first received 6 March 2019).

\section{NCT03877172 \{published data only\}}

* NCT03877172. High flow nasal cannula in thoracic surgery: a physiologic study. clinicaltrials.gov/ct2/show/ record/NCT03877172 (first received 15 March 2019). [CLINICALTRIALS.GOV: NCT03877172]

\section{NCT03928535 \{published data only\}}

* NCT03928535. Effect of postextubation high-flow nasal cannula vs noninvasive ventilation in patients with hypercapnic COPD [Effect of postextubation high-flow nasal cannula vs noninvasive ventilation on reintubation and postextubation respiratory failure in patients with hypercapnic COPD, a randomized controlled trial]. clinicaltrials.gov/ct2/show/ NCT03928535 (first received 26th April 2019).

\section{NCT03944525 \{published data only\}}

* NCT03944525. High-flow air via nasal cannula vs non-invasive continuous positive airway pressure for hypercapnic respiratory failure [High-flow air via nasal cannula versus non-invasive continuous positive airway pressure ventilation support for hypercapnic respiratory failure: the HIGH-for-HYPER study]. clinicaltrials.gov/ct2/show/NCT03944525 (first received 9th May 2019).

\section{NCT04035460 \{published data only\}}

* NCT04035460. A pilot study comparing oxygen delivery via helmet interface versus high flow nasal cannula [A pilot randomized controlled study of non-invasive oxygenation and ventilation in patients with acute hypoxemic respiratory failure (AHRF): a comparison of oxygen delivery via helmet interface versus high flow nasal cannula (HFNC)]. clinicaltrials.gov/ct2/ show/record/NCT04035460 (first received 29th July 2019). [CLINICALTRIALS.GOV: NCT04035460] 


\section{NCT04036175 \{published data only\}}

NCT04036175. Comparison of patient work of breathing and tidal volumes with high flow nasal cannula oxygen therapy and NIV (Non-Invasive Ventilation) after extubation in the ICU [Comparison of patient work of breathing and tidal volumes with high flow nasal cannula oxygen therapy and noninvasive ventilation after extubation in the ICU: a prospective, randomized, controlled study]. clinicaltrials.gov/ct2/show/ record/NCT04036175 (first received 29th July 2019).

\section{NCT04156139 \{published data only\}}

* NCT04156139. HFNC vs NPPV after early extubation for patients with COPD [High-flow nasal cannula versus noninvasive positive pressure ventilation therapy after early extubation for patients with chronic obstructive pulmonary disease]. clinicaltrials.gov/ct2/show/NCT04156139 (first received 7th November 2019).

\section{NCT04241861 \{published data only\}}

* NCT04241861. High-flow nasal cannula vs. Helmet PSV vs. Helmet CPAP during respiratory failure [Physiological comparison of high-flow nasal cannula, helmet pressure support ventilation and continuous positive airway pressure during acute hypoxemic respiratory failure: a randomized crossover study]. clinicaltrials.gov/ct2/show/record/NCT04241861 (first received 27th January 2020).

\section{NCT04253405 \{published data only\}}

* NCT04253405. RENOVATE Fibrosis: pilot study comparing HFNC versus NIPPV in acute respiratory failure in patients with pulmonary fibrosis [Multicentric randomized controlled pilot study comparing high flow nasal cannula versus noninvasive positive pressure ventilation in acute respiratory failure in patients with pulmonary fibrosis (RENOVATE Fibrosis)]. clinicaltrials.gov/ct2/show/record/NCT04253405 (first received 5 February 2020). [NATIONALTRIALS.GOV: NCT04253405]

\section{NCT04269681 \{published data only\}}

* NCT04269681. RENOVATE palliative: HFNC vs. standard respiratory support in patients with do-not-intubate order and ARF [RENOVATE palliative study: randomized controlled trial comparing high flow nasal catheter versus standard respiratory support in patients with do-not-intubate order and acute respiratory failure]. clinicaltrials.gov/ct2/show/record/ NCT04269681 (first received 17 February 2020).

\section{NCT04293991 \{published data only\}}

* NCT04293991. High flow nasal cannula in immunocompromised patient with acute respiratory failure [High flow nasal cannula versus non-invasive ventilation in prevention of intubation in immunocompromised patient with acute hypoxemic respiratory failure]. clinicaltrials.gov/ct2/ show/NCT04293991 (first received 3rd March 2020).

\section{NCT04344730 \{published data only\}}

NCT04344730. Dexamethasone and oxygen support strategies in ICU patients with Covid-19 pneumonia. clinicaltrials.gov/ct2/ show/record/NCT04344730 (first received 14 April 2020).

\section{TCTR20171106003 \{published data only\}}

* TCTR20171106003. High flow nasal oxygen for prevention of intubation in acute non-hypercapnic hypoxemic respiratory failure in immunocompromised patients, a randomized trial. apps.who.int/trialsearch/Trial2.aspx?TrialID=TCTR20171106003 (first received 7 August 2017).

UMIN000008778 \{published data only\}

UMIN000008778. Evaluation of nasal high flow oxygen therapy [Evaluation of nasal high flow oxygen therapy for severe acute hypoxemic respiratory failure]. upload.umin.ac.jp/cgi-openbin/ctr_e/ctr_view.cgi?recptno=R000010317 (first received 27 August 2012).

\section{Additional references}

\section{Afshari 2017}

Afshari A, Wetterslev J, Smith AF. Can systematic reviews with sparse data be trusted? Anaesthesia 2017;72:12-6. [PMID: 27804113]

\section{Behrendt 2000}

Behrendt CE. Acute respiratory failure in the United States: incidence and 31-day survival. Chest 2000;118(4):1100-5. [PMID: 11035684]

\section{Bello 2016}

Bello G, De Pascale G, Antonelli M. Noninvasive ventilation. Clinics in Chest Medicine 2016;37(4):711-21. [PMID: 27842751]

\section{Bland 1995}

Bland JM, Altman DG. Multiple significance tests: the Bonferroni method. BMJ 1995;310:170. [PMID: 7833759]

\section{Borenstein 2010}

Borenstein M, Hedges LV, Higgins JP, Rothstein HR. A basic introduction to fixed-effect and random-effects models for meta-analysis. Research Synthesis Methods 2010;1(2):97-111. [PMID: 26061376]

\section{Chanques 2009}

Chanques G, Constantin JM, Sauter M, Jung B, Sebbane M, Verzilli $D$, et al. Discomfort associated with under humidified high-flow oxygen therapy in critically ill patients. Intensive Care Medicine 2009;35(6):996-1003. [PMID: 19294365]

\section{Corley 2011}

Corley A, Caruana LR, Barnett AG, Tronstad O, Fraser JF. Oxygen delivery through high-flow nasal cannulae increase end-expiratory lung volume and reduce respiratory rate in post-cardiac surgical patients. British Journal of Anaesthesia 2011;107(6):998-1004. [PMID: 21908497]

\section{Costello 1995}

Costello RW, Liston R, McNicholas WT. Compliance at night with low flow oxygen therapy: a comparison of nasal cannulae and Venturi face masks. Thorax 1995;50(4):405-6. [PMID: 7785016] 


\section{Covidence [Computer program]}

Veritas Health Innovation Covidence. Veritas Health Innovation. Melbourne, Australia: Veritas Health Innovation, Accessed 09 June 2020.

\section{Curley 2015}

Curley GF, Laffy JG, Zhang H, Slutsky AS. Noninvasive respiratory support for acute respiratory failure - high flow nasal cannula oxygen or non-invasive ventilation? Journal of Thorac Disease 2015;7(7):1092-7. [PMID: 26380720]

\section{Demoule 2015}

Demoule A, Rello J. High flow oxygen cannula: the other side of the moon. Intensive Care Medicine 2015;41(9):1673-5. [PMID: 26077054]

\section{Dysart 2009}

Dysart K, Miller TL, Wolfson MR, Shaffer TH. Research in high flow therapy: mechanisms of action. Respiratory Medicine 2009;103(10):1400-5. [PMID: 19467849]

\section{Egger 1997}

Egger M, Davey Smith G, Schneider M, Minder C. Bias in meta-analysis detected by a simple, graphical test. BMJ 1997;315(7109):629-34. [PMID: 9310563]

\section{Endnote [Computer program]}

Endnote X9. Thomson Reuters, Version accessed prior to 16 January 2021. Philadelphia (PA): Thomson Reuters, 2013.

\section{Fisher \& Paykel Healthcare}

Fisher \& Paykel Healthcare (Auckland, New Zealand). www.fphcare.co.nz (accessed 16 June 2015).

\section{Gattinoni 2012}

Gattinoni L, Carlesso E, Langer T. Towards ultraprotective mechanical ventilation. Current Opinion in Anaesthesiology 2012;25(2):141-7. [PMID: 22246461]

\section{Gregoretti 2002}

Gregoretti C, Confalonieri M, Navalesi P, Squadrone V, Frigerio P, Beltrame $\mathrm{F}$, et al. Evaluation of patient skin breakdown and comfort with a new face mask for non-invasive ventilation: a multi-center study. Intensive Care Medicine 2002;28(3):278-84. [PMID: 11904656]

\section{Groves 2007}

Groves N, Tobin A. High flow nasal oxygen generates positive airway pressure in adult volunteers. Australian Critical Care 2007;20(4):126-31. [PMID: 17931878]

\section{Guyatt 2008}

Guyatt GH, Oxman AD, Kunz R, Vist GE, Falck-Ytter Y, Schunemann HJ. What is "quality of evidence" and why is it important to clinicians? BMJ 2008;336(7651):995-8. [PMID: 18456631]

\section{Hamilton Medical, Switzerland}

Hamilton Medical, Switzerland. www.hamilton-medical.com (accessed 09 June 2020).

\section{Higgins 2011}

Higgins JPT, Green S (editors). Cochrane Handbook for Systematic Reviews of Interventions. Version 5.1.0 (updated March 2011). The Cochrane Collaboration, 2011. Available from training.cochrane.org/handbook/archive/v5.1.

\section{Higgins 2019}

Higgins JPT, Thomas J, Chandler J, Cumpston M, Li T, Page MJ, et al (editors). Cochrane Handbook for Systematic Reviews of Interventions. 2nd edition. Chichester (UK): John Wiley \& Sons, 2019.

\section{Huang 2018}

Huang H-W, Sun X-M, Shi Z-H, Chen G-Q, Chen L, Friedrich JO, et al. Effect of high-flow nasal cannula oxygen therapy versus conventional oxygen therapy and noninvasive ventilation on reintubation rate in adult patients after extubation: a systematic review and meta-analysis of randomized controlled trials. Journal of Intensive Care Medicine 2018;33(11):609-23. [PMID: 28429603]

\section{Kernick 2010}

Kernick J, Magarey J. What is the evidence for the use of high flow nasal cannula oxygen in adult patients admitted to critical care units? A systematic review. Australian Critical Care 2010;23:53-70. [PMID: 20206546]

\section{Lellouche 2002}

Lellouche F, Maggiore SM, Deye N, Taille S, Pigeot J, Harf A, et al. Effect of the humidification device on the work of breathing during noninvasive ventilation. Intensive Care Medicine 2002;28(11):1582-9. [PMID: 12415444]

\section{Levy 2016}

Levy SD, Alladina JW, Hibbert KA, Harris RS, Bajwa EK, Hess DR. High-flow oxygen therapy and other inhaled therapies in intensive care units. Lancet 2016;387(10030):1867-78. [PMID: 27203510]

\section{Lewis 2019}

Lewis SR, Pritchard MW, Thomas CM, Smith AF. Pharmacological agents for adults with acute respiratory distress syndrome. Cochrane Database of Systematic Reviews 2019, Issue 7. Art. No: CD004477. [DOI: 10.1002/14651858.CD004477.pub3]

\section{Maitra 2016}

Maitra S, Som A, Bhattacharjee S, Arora MK, Baidya DK. Comparison of high-flow nasal oxygen therapy with conventional oxygen therapy and noninvasive ventilation in adult patients with acute hypoxemic respiratory failure: a meta-analysis and systematic review. Journal of Critical Care 2016;35:138-44. [PMID: 27481749]

\section{Mayfield 2014}

Mayfield S, Jauncey-Cooke J, Hough JL, Schibler A, Gibbons K, Bogossian F. High-flow nasal cannula therapy for respiratory support in children. Cochrane Database of Systematic Reviews 2014, Issue 3. Art. No: CD009850. [DOI: 10.1002/14651858.CD009850.pub2] 


\section{Mehta 2001}

Mehta S, Hill NS. Noninvasive ventilation. American Journal of Respiratory and Critical Care Medicine 2001;163(2):540-77. [PMID: 11179136]

\section{Merck Sharp \& Dohome}

Merck Sharpe \& Dohome, USA. www.msd-uk.com/index.xhtml (accessed 8 June 2020).

\section{Monro-Somerville 2017}

Monro-Somerville T, Sim M, Ruddy J, Vilas M, Gillies MA. The effect of high-flow nasal cannula oxygen therapy on mortality and intubation rate in acute respiratory failure. Critical Care Medicine 2017;45(4):e449-56. [PMID: 27611978]

\section{Morandi 2011}

Morandi A, Brummel NE, Ely EW. Sedation, delirium and mechanical ventilation: the 'ABCDE' approach. Current Opinion in Critical Care 2011;17(1):43-9. [PMID: 21169829]

\section{Muscadere 2008}

Muscedere J, Dodek P, Keenan S, Fowler R, Cook D, Heyland D. Comprehensive evidence-based clinical practice guidelines for ventilator-associated pneumonia: diagnosis and treatment. Journal of Critical Care 2008;23:138-47. [PMID: 18359431]

\section{Nava 2009}

Nava S, Hill N. Non-invasive ventilation in acute respiratory failure. Lancet 2009;374(9685):250-9. [PMID: 19616722]

\section{Nedel 2017}

Nedel WL, Deutschendorf C, Moraes RFE. High-flow nasal cannula in critically ill subjects with or at risk for respiratory failure: a systematic review and meta-analysis. Respiratory Care 2017;62(1):123-32. [PMID: 27879383]

\section{Nelson 2000}

Nelson BJ, Weinert CR, Bury CL, Marinelli WA, Gross CR. Intensive care unit drug use and subsequent quality of life in acute lung injury patients. Critical Care Medicine 2000;28(11):3626-30. [PMID: 11098964]

\section{Ni 2018}

Ni Y-N, Luo J, Yu H, Liu D, Liang B-M, Liang Z-A. The effect of high-flow nasal cannula in reducing the mortality and the rate of endotracheal intubation when used before mechanical ventilation compared with conventional oxygen therapy and noninvasive positive pressure ventilation. A systematic review and meta-analysis. American Journal of Emergency Medicine 2018;36(2):226-33. [PMID: 28780231]

\section{Nishimura 2015}

Nishimura M. High-flow nasal cannula oxygen therapy in adults. Journal of Intensive Care 2015;3(1):15. [PMID: 25866645]

\section{O'Driscoll 2008}

O'Driscoll BR, Howard LS, Davison AG. British Thoracic Society Guideline for emergency oxygen use in adult patients. Thorax 2008;63 Suppl 6:vi1-68. [PMID: 18838559]

\section{Papazian 2016}

Papazian L, Corley A, Hess D, Fraser JF, Frat J-P, Guitton C, et al. Use of high-flow nasal cannula oxygenation in ICU adults: a narrative review. Intensive Care Medicine 2016;42(9):1336-49. [PMID: 26969671]

\section{Parke 2009}

Parke R, McGuinness S, Eccleston M. Nasal high-flow therapy delivers low level positive airway pressure. British Journal of Anaesthesia 2009;103(6):886-90. [PMID: 19846404]

\section{Price 2008}

Price AM, Plowright C, Makowski A, Misztal B. Using a highflow respiratory system (Vapotherm) within a high dependency setting. Nursing and Critical Care 2008;13(6):298-304. [PMID: 19128313]

\section{ResMed USA}

ResMed, USA. www.resmed.com/en-us/ (accessed 09 June 2020).

\section{RevMan Web 2019 [Computer program]}

Cochrane Collaboration Review Manager Web (RevMan Web). Version accessed prior to 16 January 2021. Cochrane Collaboration, 2019. Available at revman.cochrane.org.

\section{Roca 2010}

Roca O, Riera J, Torres F, Masclans JR. High-flow oxygen therapy in acute respiratory failure. Respiratory Care 2010;55(4):408-13. [PMID: 20406507]

\section{Rochwerg 2019}

Rochwerg B, Granton D, Wang DX, Helviz Y, Einav S, Frat JP, et al. High flow nasal cannula compared with conventional oxygen therapy for acute hypoxemic respiratory failure: a systematic review and meta-analysis. Intensive Care Medicine 2019;45(5):563-72. [PMID: 30888444]

\section{Safdar 2005}

Safdar N, Dezfulian C, Collard HR, Saint S. Clinical and economic consequences of ventilator-associated pneumonia: a systematic review. Critical Care Medicine 2005;33(10):2184-93. [PMID: 16215368]

\section{Salah 1988}

Salah B, Dinh Xuan AT, Fouilladieu JL, Lockhart A, Regnard J. Nasal mucociliary transport in healthy subjects is slower when breathing dry air. European Respiratory Journal 1988;1(9):852-5. [PMID: 3229484]

\section{Sasaki 2003}

Sasaki H, Yamakage M, Iwasaki S, Mizuuchi M, Namiki A. Design of oxygen delivery systems influences both effectiveness and comfort in adult volunteers. Canadian Journal of Anaesthesia 2003;50(10):1052-5. [PMID: 14656787]

\section{Shelly 1999}

Shelly MP, Nightingale P. ABC of intensive care: respiratory support. BMJ 1999;318(7199):1674-7. [PMID: 10373174] 


\section{Sztrymf 2011}

Sztrymf B, Messika J, Bertrand F, Hurel D, Leon R, Dreyfuss D, et al. Beneficial effects of humidified high flow nasal oxygen in critical care patients: a prospective pilot study. Intensive Care Medicine 2011;37(11):1780-6. [PMID: 21946925]

\section{Sztrymf 2011a}

Sztrymf B, Messika J, Mayot T, Lenglet H, Dreyfuss D, Ricard JD. Impact of high-flow nasal cannula oxygen therapy on intensive care unit patients with acute respiratory failure: a prospective observational study. Journal of Critical Care 2012;27(3):e9-13. [PMID: 21958974]

\section{Wang 2020}

Wang Y, Ni Y, Sun J, Liang Z. Use of high-flow nasal cannula for immunocompromise and acute respiratory failure: a systematic review and meta-analysis. Journal of Emergency Medicine 2020;58(3):413-23. [PMID: 32220545]

\section{Wilkinson 2016}

Wilkinson D, Andersen C, O'Donnell CPF, De Paoli AG, Manley BJ. High flow nasal cannula for respiratory support in preterm infants. Cochrane Database of Systematic Reviews 2016, Issue 2. Art. No: CD006405. [DOI: 10.1002/14651858.CD006405.pub3]

\section{Xu 2018}

Xu Z, Li Y, Zhou J, Li X, Huang Y, Liu X, et al. High-flow nasal cannula in adults with acute respiratory failure and after extubation: a systematic review and meta-analysis. Respiratory Research 2018;19(1):202. [PMID: 30326893]

\section{Zevola 2001}

Zevola DR, Maier CB. Use of nasal cannula versus face mask after extubation in patients after cardiothoracic surgery. Critical Care Nurse 2001;21(3):47-53. [PMID: 11858676]

\section{CHARACTERISTICS OF STUDIES}

Characteristics of included studies [ordered by study ID]

\section{Zhao 2017}

Zhao H, Wang H, Sun F, Lyu S, An Y. High-flow nasal cannula oxygen therapy is superior to conventional oxygen therapy but not to noninvasive mechanical ventilation on intubation rate: a systematic review and meta-analysis. Critical Care 2017;21(1):184. [PMID: 28701227]

\section{Zhu 2016}

Zhu Y, Yin H, Zhang R, Wei J. High flow nasal cannula oxygen therapy versus conventional oxygen therapy in cardiac surgical patients: a meta-analysis. Journal of Critical Care 2016;38:123-8. [PMID: 27886577]

\section{References to other published versions of this review}

\section{Corley 2012}

Corley A, Rickard CM, Aitken LM, Johnston A, Barnett A, Fraser JF. High flow nasal cannulae for respiratory support in adult intensive care patients. Cochrane Database of Systematic Reviews 2012, Issue 11. Art. No: CD010172. [DOI: 10.1002/14651858.CD010172]

\section{Corley 2017}

Corely A, Rickard CM, Aitken LM, Johnston A, Barnett A, Fraser JF, et al. High-flow nasal cannulae for respiratory support in adult intensive care patients. Cochrane Database of Systematic Reviews 2017, Issue 5. Art. No: CD010172. [DOI: 10.1002/14651858.CD010172.pub2]

* Indicates the major publication for the study

Azoulay 2018

\section{Study characteristics}

Methods RCT, parallel-group design. Multicentre study

Participants

Total number of randomized participants: 778

Setting: 32 ICUs; France

Inclusion criteria: ICU admission; $\geq 18$ years of age; $\mathrm{AHRF}$ with $\mathrm{PaO}_{2}<60 \mathrm{mmHg}$ or $\mathrm{SpO}_{2}<90 \%$ on room air, or tachypnoea $>30$ breaths/min or laboured breathing or respiratory distress; need for oxygen flow of $\geq 6 \mathrm{~L} / \mathrm{min}$; known immunosuppression; written informed consent

Exclusion criteria: people with AIDS; imminent death; refusal to participate in study; anatomical factors precluding use of nasal cannula; hypercapnia indicting NIV; isolated cardiogenic pulmonary oedema indicating NIV; pregnancy or breastfeeding; absence of health insurance coverage; surgery within the last 6 days

\section{Baseline characteristics:}

Intervention group (HFNC): 
Azoulay 2018 (Continued)

- Age, median (IQR): 64 (55 to 70) years

- Gender, M/F: 270/118

- BMI, mean (SD): not reported

- SOFA, median (IQR) : 6 (4 to 8)

- SAPS II, median (IQR): 36 (28 to 46)

- $\mathrm{PaCO}_{2}$, mean (SD): not reported

- $\mathrm{PaO}_{2} / \mathrm{FiO}_{2}$, median (IQR): 136 (96 to 187) mmHg

- Respiratory rate, median (IQR): 33 (28 to 39 ) breaths/min

Control group (standard oxygen therapy):

- Age, median (IQR): 63 (56 to 71 ) years

- Gender, M/F: 247/141

- BMI, mean (SD): not reported

- SOFA, median (IQR): 6 (4 to 8)

- SAPS II, median (IQR): 37 (28 to 48)

- $\mathrm{PaCO}_{2}$, mean (SD): not reported

- $\mathrm{PaO}_{2} / \mathrm{FiO}_{2}$, median (IQR): 128 (92 to 164 ) mmHg

- Respiratory rate, median (IQR): 32 (27 to 38 ) breaths/min

- Randomized, $\mathrm{n}=389$; losses, $\mathrm{n}=1$ (withdrew consent), 13 did not receive intervention as randomized; analysed, $\mathrm{n}=388$

- Details: started within 15 minutes of randomization, and for whole duration of ICU stay. Flow initiated at $50 \mathrm{~L} / \mathrm{min}$ and $100 \% \mathrm{FiO}_{2}$, then subsequent flow to achieve $\mathrm{SpO}_{2} \geq 95 \%$ up to $\geq 50 \mathrm{~L} / \mathrm{min}$ within the first 3 days then up to $60 \mathrm{~L} / \mathrm{min}$ as needed. If participants needed MV, HFNC was used during laryngoscopy and immediately after extubation. Standard oxygen therapy was only used if significant nasal discomfort or skin breakdown.

Control group (standard oxygen):

- Randomized, $n=389$; losses, $n=1$ (withdrew consent), 31 did not receive intervention as randomized, 30 received HFNC; analysed, $n=388$

- Details: started within 15 minutes of randomization, and for whole duration of ICU stay. Oxygen given by any device or combination of devices (nasal prongs or mask with or without reservoir bag and with or without Venturi system). Flow to achieve target $\mathrm{SpO}_{2} \geq 95 \%$. HFNC only given if participants had a do-not-intubate order or for whom standard oxygen had failed. NIV only used as long as hypercapnia or pulmonary oedema were present.

Outcomes

Mortality within 28 days; hospital and ICU mortality; number needing MV by day 28; respiratory rate (normal values, 12-20), lowest $\mathrm{PaO}_{2} / \mathrm{FiO}_{2}$, patient comfort score (range 0 to $10=$ severe discomfort to perfect comfort); dyspnoea score (range 0 to 10 = no dyspnoea to severe dyspnoea); ICU and hospital lengths of stay; incidence of ICU-acquired infections

Notes

Funding/declarations of interest: funded by the French Ministry of Health. Supplies for high-flow oxygen from Fisher \& Paykel Healthcare Ltd. Funders had no role in the design and conduct of the study, nor in preparation of the manuscript etc.

Some authors received fees from one or more of: Gilead; Astellas; Baxter; Alexion; Ablynx; Merk Sharp and Dohme, Fisher \& Paykel Healthcare, Xenios; Boehringer Ingelheim; Pfizer; Astute; Bristol-Myers Squibb; Jazz Pharma; Sanofi-Aventis; Resmed; Philips; Hamilton; Medtronic; French Ministry of Health. One author serves on a data and safety monitoring board for the French Ministry of Health.

Study dates: 19 May 2016 to 31 December 2017

\section{Risk of bias}


Azoulay 2018 (Continued)

\begin{tabular}{|c|c|c|}
\hline Bias & Authors' judgement & Support for judgement \\
\hline $\begin{array}{l}\text { Random sequence genera- } \\
\text { tion (selection bias) }\end{array}$ & Low risk & Use of an electronic system \\
\hline $\begin{array}{l}\text { Allocation concealment } \\
\text { (selection bias) }\end{array}$ & Low risk & $\begin{array}{l}\text { Quote: "Randomization was achieved using an electronic system incorporated } \\
\text { in the electronic case report from to ensure allocation concealment". }\end{array}$ \\
\hline $\begin{array}{l}\text { Blinding of participants } \\
\text { and personnel (perfor- } \\
\text { mance bias) } \\
\text { All outcomes }\end{array}$ & Unclear risk & $\begin{array}{l}\text { Blinding was not possible. Although we expected that this would not influence } \\
\text { outcome data, we could not be certain of this. }\end{array}$ \\
\hline $\begin{array}{l}\text { Blinding of outcome as- } \\
\text { sessors (objective out- } \\
\text { comes) }\end{array}$ & Low risk & $\begin{array}{l}\text { Quote: "No blinding of adjudication was performed for outcome assess- } \\
\text { ments". The assessors were unblinded; we did not anticipate that this would } \\
\text { influence the assessment of objective outcome measures. }\end{array}$ \\
\hline $\begin{array}{l}\text { Blinding of outcome as- } \\
\text { sessors (subjective mea- } \\
\text { sures) }\end{array}$ & Low risk & $\begin{array}{l}\text { Participants were the outcome assessors for comfort and dyspnoea on a stan- } \\
\text { dardized scale: we did not anticipate that this would influence the assessment } \\
\text { of these outcome measures. }\end{array}$ \\
\hline $\begin{array}{l}\text { Incomplete outcome data } \\
\text { (attrition bias) } \\
\text { All outcomes }\end{array}$ & Low risk & Loss of only two participants \\
\hline
\end{tabular}

\begin{tabular}{ll}
\hline $\begin{array}{l}\text { Selective reporting (re- } \\
\text { porting bias) }\end{array}$ & Low risk \\
& $\begin{array}{l}\text { Study was prospectively registered with a clinical trials register } \\
\text { (NCT02739451). Outcomes relevant to the review were reported as described } \\
\text { in the clinical trials register. }\end{array}$
\end{tabular}

Other bias Low risk We identified no other sources of bias.

Brainard 2017

\section{Study characteristics}

\begin{tabular}{|c|c|}
\hline Methods & RCT, parallel-group design. Single-centre study \\
\hline \multirow[t]{11}{*}{ Participants } & Total number of randomized participants: 51 \\
\hline & Setting: ICU, USA \\
\hline & $\begin{array}{l}\text { Inclusion criteria: } \geq 18 \text { years of age; undergoing thoracic surgery with scheduled admission to the ICU } \\
\text { postoperatively }\end{array}$ \\
\hline & $\begin{array}{l}\text { Exclusion criteria: }<18 \text { years of age; pregnant or breastfeeding; known diagnosis of obstructive sleep } \\
\text { apnoea; current or pervious lung transplantation; previous pneumonectomy; home oxygen }>4 \mathrm{~L} / \mathrm{min} \text {; } \\
\text { inability to adhere to assigned treatment for the intended duration }\end{array}$ \\
\hline & Baseline characteristics (for those who continued treatment): \\
\hline & Intervention group (HFNC): \\
\hline & - Age, mean (SD): $57( \pm 14)$ years \\
\hline & - Gender, M/F: 8/10 \\
\hline & - BMI, mean (SD): $26( \pm 5) \mathrm{kg} / \mathrm{m}^{2}$ \\
\hline & - ASA II/III/IV: 5/12/1 \\
\hline & - SAPS II, mean (SD): $19( \pm 7)$ \\
\hline
\end{tabular}


Brainard 2017 (Continued)

- $\mathrm{PaCO}_{2}$, mean (SD): not reported

- $\mathrm{PaO}_{2} / \mathrm{FiO}_{2}$, mean (SD): not reported

Control group (standard oxygen):

- Age, mean (SD): 59 ( \pm 16 ) years

- Gender, M/F: 14/12

- BMI, mean (SD): $25( \pm 5) \mathrm{kg} / \mathrm{m}^{2}$

- ASA II/III/IV: $1 / 19 / 1$

- SAPS II, mean (SD): 23 ( \pm 7$)$

- $\mathrm{PaCO}_{2}$, mean (SD): not reported

- $\mathrm{PaO}_{2} / \mathrm{FiO}_{2}$, mean (SD): not reported

Interventions Intervention group (HFNC):

- Randomized, $\mathrm{n}=25$; losses, $\mathrm{n}=7$ (discontinued due to discomfort); analysed, $\mathrm{n}=18$ (we included 7 lost participants as outcome data for comfort)

- Details: HFNC using MaxVenturi, started after transfer to ICU, following surgery, set flow of $40 \mathrm{~L} / \mathrm{min}$, $\mathrm{FiO}_{2}$ titrated by respiratory therapists to maintain $\mathrm{SpO}_{2} \geq 90 \%$. Therapy continued for 48 hours or until transfer from the ICU to a ward

Control group (standard oxygen):

- Randomized, $\mathrm{n}=26$; losses, $\mathrm{n}=0$; analysed, $\mathrm{n}=26$

- Details: standard oxygen given via nasal cannula or face mask titrated by nurses as required to maintain $\mathrm{SpO}_{2} \geq 90 \%$. Therapy continued for 48 hours or until transfer from the ICU to a ward

Outcomes

Composite of postoperative pulmonary outcomes (severe hypoxaemia, acute respiratory failure, escalation of therapy to non-invasive ventilation, re-intubation, occurrence of hospital-acquired pneumonia); ICU and hospital lengths of stay; postoperative oxygenation

Note: study authors did not separately report data for the each event in the primary outcome.

Notes

Funding/declarations of interest: one study author was supported by the NIS/National Institute on Drug Abuse. Study authors declared no conflicts of interest.

Study dates: August 2013 to June 2015

\section{Study characteristics}

Methods RCT, cross-over study. Single-centre study

Setting: medical-surgical ICU; Montpelier, France

Inclusion criteria: $\geq 18$ years old hospitalized in a medical-surgical ICU, planned for tracheostomy tube removal which was placed in the ICU for weaning from mechanical ventilation

Exclusion criteria: pregnancy, adult under tutelage, contraindications for NIV

\section{Baseline characteristics (all patients):}

- Age: 54 to 66 years

- Respiratory rate, median (IQR): 18 (22 to 20 ) breaths/min 
Chanques 2013 (Continued)

- $\mathrm{PaCO}_{2}$ : not reported

- $\mathrm{PaO}_{2} / \mathrm{FiO}_{2}$ : not reported

Interventions

Flow rates of 15,30 , and 45 litres per minute were tested in a randomized order for each device.

- High-flow face mask with a reservoir bag

- Optiflow high-flow nasal cannulae

- Boussignac oxygen therapy system\&

For each device and flow rate, participants were asked to have their mouth open and mouth closed in a randomized order. Each device was used for 5 minutes, with 15-minute washout between treatments.

\begin{tabular}{ll}
\hline Outcomes & Tracheal pressure, $\mathrm{FiO}_{2}$ delivered, respiratory discomfort, respiratory rate (at end of each treatment pe- \\
riod), noise intensity
\end{tabular}
riod), noise intensity

\section{Notes}

Funding sources/declarations of interest: Study authors disclosed funding of $€ 3000$ from Fisher \& Paykel Healthcare Ltd, France, which was used to acquire technical equipment and clinical research insurance, and to present results at scientific meetings.

Study dates: not reported

\section{Risk of bias}

\begin{tabular}{|c|c|c|}
\hline Bias & Authors' judgement & Support for judgement \\
\hline $\begin{array}{l}\text { Random sequence genera- } \\
\text { tion (selection bias) }\end{array}$ & Unclear risk & Method of randomization not stated \\
\hline $\begin{array}{l}\text { Allocation concealment } \\
\text { (selection bias) }\end{array}$ & Unclear risk & Method of allocation concealment not stated \\
\hline $\begin{array}{l}\text { Blinding of participants } \\
\text { and personnel (perfor- } \\
\text { mance bias) } \\
\text { All outcomes }\end{array}$ & Unclear risk & $\begin{array}{l}\text { Blinding was not possible. Although we expected that this would not influence } \\
\text { outcome data, we could not be certain of this. }\end{array}$ \\
\hline $\begin{array}{l}\text { Blinding of outcome as- } \\
\text { sessors (objective out- } \\
\text { comes) }\end{array}$ & Low risk & $\begin{array}{l}\text { Investigators were outcome assessors for objective outcomes, but standard- } \\
\text { ized tools were used for measurement, reducing risk of bias. }\end{array}$ \\
\hline $\begin{array}{l}\text { Blinding of outcome as- } \\
\text { sessors (subjective mea- } \\
\text { sures) }\end{array}$ & Low risk & $\begin{array}{l}\text { Participants were outcome assessors for respiratory and auditory discomfort } \\
\text { on a standardized scale. We did not think this would influence the subjective } \\
\text { outcome data. }\end{array}$ \\
\hline \multirow[t]{2}{*}{$\begin{array}{l}\text { Incomplete outcome data } \\
\text { (attrition bias) } \\
\text { All outcomes }\end{array}$} & Unclear risk & $\begin{array}{l}\text { One participant was excluded owing to major intolerance to the device but } \\
\text { possibly should have been regarded as a treatment failure. In such a small } \\
\text { study, this is likely to have had an effect. }\end{array}$ \\
\hline & & $\begin{array}{l}\text { Owing to inability of } 4 \text { participants in the Boussignac group to adhere to the } \\
\text { protocol, it is likely that data were incomplete; however it was not mentioned } \\
\text { how this was handled in the analysis. }\end{array}$ \\
\hline $\begin{array}{l}\text { Selective reporting (re- } \\
\text { porting bias) }\end{array}$ & Unclear risk & $\begin{array}{l}\text { ISRCTN15995925. Retrospectively registered in August 2012. Not possible to } \\
\text { establish any reporting bias through comparison with the trial register proto- } \\
\text { col }\end{array}$ \\
\hline Other bias & Low risk & We identified no other risks of bias. \\
\hline
\end{tabular}




\section{Study characteristics}

\begin{tabular}{|c|c|}
\hline Methods & RCT, parallel-group design. Single-centre study \\
\hline Participants & 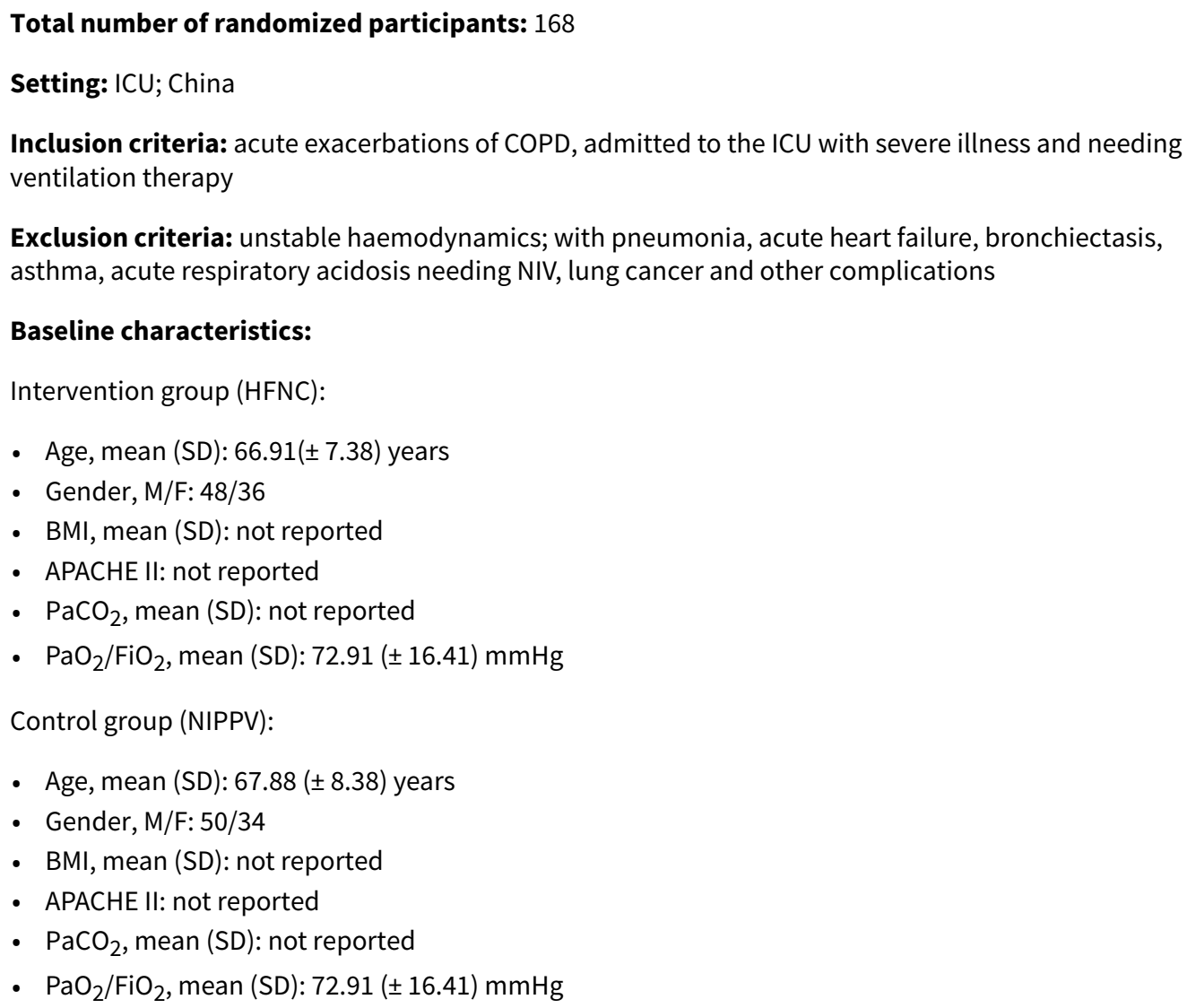 \\
\hline
\end{tabular}

Interventions

Intervention group (HFNC):

- Randomized, $n=84$; losses, $n=0$; analysed, $n=84$

- Details: HFNC with $\mathrm{OH}-60 \mathrm{C}$ high-flow noninvasive breathing apparatus (Micomme, Hunan, China). Temperature set at $37^{\circ} \mathrm{C}$, flow rate of 30 to $35 \mathrm{~L} / \mathrm{min}$

Control group (NIPPV):

- Randomized, $\mathrm{n}=84$; losses, $\mathrm{n}=0$; analysed, $\mathrm{n}=84$

- Details: ventilated by mouth and nose using Hamilton G5 ventilator (Hamilton Medical, Switzerland), initial inspiratory positive airway pressure set at $10 \mathrm{~cm} \mathrm{H}_{2} \mathrm{O}$ and expiration pressure set at $5 \mathrm{~cm} \mathrm{H}_{2} \mathrm{O}$. $\mathrm{FiO}_{2}$ adjusted to ensure oxygen saturation

Outcomes Blood gases $\left(\mathrm{PaO}_{2} ; \mathrm{PaCO}_{2} ; \mathrm{pH} ; \mathrm{SpO}_{2}\right)$ at 12 hours and 5 days after therapy; ventilation support time; length of hospital stay; complications; comfort; nursing satisfaction

Notes Funding/declarations of interest: funding not reported. Study authors declared no competing interests.

Study dates: January 2015 to December 2017

\section{Risk of bias}

High-flow nasal cannulae for respiratory support in adult intensive care patients (Review) 
Cong 2019 (Continued)

\begin{tabular}{lll} 
Bias & Authors' judgement & Support for judgement \\
\hline $\begin{array}{l}\text { Random sequence genera- } \\
\text { tion (selection bias) }\end{array}$ & Unclear risk & Described as randomized, but no additional details \\
\hline $\begin{array}{l}\text { Allocation concealment } \\
\text { (selection bias) }\end{array}$ & Unclear risk & No details
\end{tabular}

\begin{tabular}{|c|c|c|}
\hline $\begin{array}{l}\text { Blinding of participants } \\
\text { and personnel (perfor- } \\
\text { mance bias) } \\
\text { All outcomes }\end{array}$ & Unclear risk & $\begin{array}{l}\text { Blinding was not possible. Although we expected that this would not influence } \\
\text { outcome data, we could not be certain of this. }\end{array}$ \\
\hline
\end{tabular}

\begin{tabular}{|c|c|c|}
\hline $\begin{array}{l}\text { Blinding of outcome as- } \\
\text { sessors (objective out- } \\
\text { comes) }\end{array}$ & Low risk & $\begin{array}{l}\text { Blinding of outcome assessors was not described; we did not anticipate that } \\
\text { this would influence the assessment of objective outcome measures. }\end{array}$ \\
\hline
\end{tabular}

\begin{tabular}{|c|c|c|}
\hline $\begin{array}{l}\text { Blinding of outcome as- } \\
\text { sessors (subjective mea- } \\
\text { sures) }\end{array}$ & Low risk & $\begin{array}{l}\text { Participants were outcome assessors for comfort using a standardized scale: } \\
\text { we did not anticipate that this would influence the assessment of this out- } \\
\text { come measure. }\end{array}$ \\
\hline $\begin{array}{l}\text { Incomplete outcome data } \\
\text { (attrition bias) } \\
\text { All outcomes }\end{array}$ & Low risk & No apparent losses \\
\hline $\begin{array}{l}\text { Selective reporting (re- } \\
\text { porting bias) }\end{array}$ & Unclear risk & $\begin{array}{l}\text { Study authors did not report prepublished protocol or clinical trials registra- } \\
\text { tion. it was not feasible to effectively assess risk of selective reporting bias } \\
\text { without these documents. }\end{array}$ \\
\hline Other bias & Low risk & We identified no other sources of bias. \\
\hline
\end{tabular}

Corley 2014

\section{Study characteristics}

\begin{tabular}{ll}
\hline Methods & RCT, parallel-group design. Single-centre study \\
\hline
\end{tabular}

Participants

\section{Total number of participants: 155}

Setting: ICU; Brisbane, Australia

Inclusion criteria: $\geq 18$ years, $\mathrm{BMI} \geq 30 \mathrm{~kg} / \mathrm{m}^{2}$, scheduled to undergo cardiac surgery on cardiopulmonary bypass

Exclusion criteria: ventilation time $>36$ hours, extubation onto NIPPV, requirement for tracheostomy, extubation as part of end-of-life treatment

\section{Baseline characteristics:}

Intervention group (HFNC):

- Age mean (SD): $63( \pm 11.4)$ years

- Gender, M/F: 58/23

- BMI, mean (SD): $36( \pm 5.2) \mathrm{kg} / \mathrm{m}^{2}$

- APACHE II, mean (SD): 15 (3.6)

- Respiratory rate: not reported 
Corley 2014 (Continued)

- $\mathrm{PaCO}_{2}:$ not reported

- $\mathrm{PaO}_{2} / \mathrm{FiO}_{2}$ : not reported

Control group (standard oxygen therapy):

- Age mean (SD): $65( \pm 11.1)$ years

- Gender, M/F: 56/18

- BMI, mean (SD): $35( \pm 4.3) \mathrm{kg} / \mathrm{m}^{2}$

- APCAHE II, mean (SD): 15 (3.9)

- Respiratory rate: not reported

- $\mathrm{PaCO}_{2}$ : not reported

- $\mathrm{PaO}_{2} / \mathrm{FiO}_{2}$ : not reported

Interventions

Interventions group (HFNC):

- Randomized, $\mathrm{n}=81$; losses, $\mathrm{n}=0$; analysed, $\mathrm{n}=81$

- Details: Optiflow; Fisher \& Paykel Healthcare Ltd; humidified to $37^{\circ} \mathrm{C}$, flow rate commenced at $35 \mathrm{~L} /$ min, then titrated to a maximum of $50 \mathrm{~L} / \mathrm{min}$; $\mathrm{FiO}_{2}$ titrated to maintain $\mathrm{SpO}_{2} \geq 95 \%$ for 8 hours, with short breaks for nasal care or mobilisation

Control group (standard oxygen therapy):

- Randomized, $\mathrm{n}=74$; losses, $\mathrm{n}=0$; analysed, $\mathrm{n}=74$

- Details: Oxygen delivered at 2 to $4 \mathrm{~L} / \mathrm{min}$ via nasal cannulae or $6 \mathrm{~L} / \mathrm{min}$ via simple face mask titrated to maintain $\mathrm{SpO}_{2} \geq 95 \%$

Both applied after extubation

Outcomes Atelectasis on chest X-ray, oxygenation $\left(\mathrm{PaO}_{2} / \mathrm{FiO}_{2}\right)$, respiratory rate, subjective dyspnoea, failure of allocated treatment

Notes

Funding/declarations of interest: unrestricted grant from Fisher \& Paykel Healthcare Ltd; two study authors received travel and accommodation support from Fisher \& Paykel Healthcare Ltd; manufacturer had no part in study design, data collection, data analysis, or creation of the manuscript.

Study dates: February 2011 to March 2012

\section{Risk of bias}

\begin{tabular}{|c|c|c|}
\hline Bias & Authors' judgement & Support for judgement \\
\hline $\begin{array}{l}\text { Random sequence genera- } \\
\text { tion (selection bias) }\end{array}$ & Low risk & Computerised random numbers table in blocks of 8 \\
\hline $\begin{array}{l}\text { Allocation concealment } \\
\text { (selection bias) }\end{array}$ & Low risk & Use of numbered, opaque envelopes to maintain allocation concealment \\
\hline $\begin{array}{l}\text { Blinding of participants } \\
\text { and personnel (perfor- } \\
\text { mance bias) } \\
\text { All outcomes }\end{array}$ & Unclear risk & $\begin{array}{l}\text { Blinding was not possible. Although we expected that this would not influence } \\
\text { outcome data, we could not be certain of this. }\end{array}$ \\
\hline $\begin{array}{l}\text { Blinding of outcome as- } \\
\text { sessors (objective out- } \\
\text { comes) }\end{array}$ & Low risk & $\begin{array}{l}\text { Primary outcome assessment (atelectasis) blinded, but other outcome assess- } \\
\text { ment not blinded. We did not anticipate that this would influence the assess- } \\
\text { ment of objective outcome measures. }\end{array}$ \\
\hline
\end{tabular}


Corley 2014 (Continued)

Blinding of outcome as- Low risk Participants were outcome assessors for subjective dyspnoea: we did not exsessors (subjective meapect that this would influence the assessment of this outcome measure. sures)

\begin{tabular}{lll}
$\begin{array}{l}\text { Incomplete outcome data } \\
\text { (attrition bias) } \\
\text { All outcomes }\end{array}$ & Low risk & No losses \\
\hline $\begin{array}{l}\text { Selective reporting (re- } \\
\text { porting bias) }\end{array}$ & Low risk & $\begin{array}{l}\text { ACTRN12610000942055. Prospective trial registration. All outcomes reported } \\
\text { as stated in trial registry }\end{array}$
\end{tabular}

Other bias Low risk We identified no other risks of bias.

\section{Cuquemelle 2012}

\section{Study characteristics}

\begin{tabular}{|c|c|}
\hline Methods & RCT, parallel-group design. Single-centre study \\
\hline Participants & 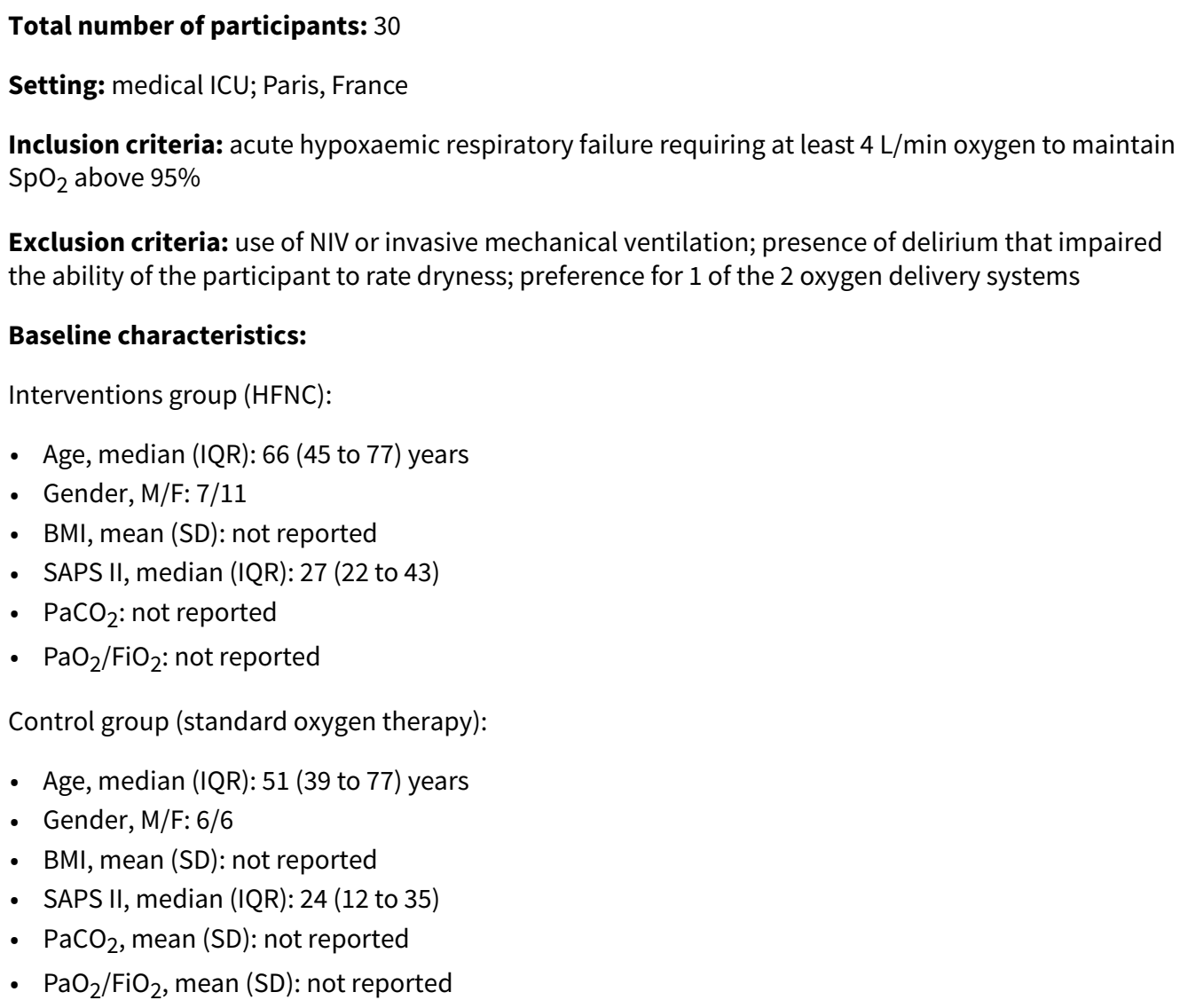 \\
\hline
\end{tabular}

- Randomized, $\mathrm{n}=19$; losses, $\mathrm{n}=1$ (worsened); analysed, $\mathrm{n}=18$

- Details: Optiflow; Fisher \& Paykel Healthcare; humidified to $37^{\circ} \mathrm{C}$, flow rate at $40 \mathrm{~L} / \mathrm{min}$

Control group (standard oxygen therapy): 
- Randomized, $\mathrm{n}=12$; losses, $\mathrm{n}=6$ ( 4 worsened, 2 rapidly recovered); analysed, $\mathrm{n}=12$

- Use of a flow meter from wall oxygen without humidification

- Randomized to receive therapy during first 24 hours, then crossed over to alternative therapy for 4 hours to reduce dropouts

\begin{tabular}{ll}
\hline Outcomes & Nasal airway calibre was measured by acoustic rhinometry at baseline, after 4 and 24 hours, and 4 \\
& hours after cross-over. Dryness of the nose, mouth, and throat was auto-evaluated and was assessed \\
blindly by an otorhinolaryngologist. After cross-over, participants were asked which system they pre- \\
ferred.
\end{tabular}

Notes $\quad$ Funding/declarations of interest: received a research grant from Fisher \& Paykel Healthcare Ltd, but manufacturers had no part in the analysis of results or writing of the paper.

Study dates: December 2009 to December 2010

Note: we noted potentially clinically important differences in baseline characteristics. Specifically, participants in the intervention group were older and had higher rates of infectious pneumonia.

\section{Risk of bias}

\begin{tabular}{|c|c|c|}
\hline Bias & Authors' judgement & Support for judgement \\
\hline $\begin{array}{l}\text { Random sequence genera- } \\
\text { tion (selection bias) }\end{array}$ & Unclear risk & Not stated in the paper \\
\hline $\begin{array}{l}\text { Allocation concealment } \\
\text { (selection bias) }\end{array}$ & Unclear risk & Not stated in the paper \\
\hline $\begin{array}{l}\text { Blinding of participants } \\
\text { and personnel (perfor- } \\
\text { mance bias) } \\
\text { All outcomes }\end{array}$ & Unclear risk & $\begin{array}{l}\text { Blinding was not possible. Although we expected that this would not influence } \\
\text { outcome data, we could not be certain of this. }\end{array}$ \\
\hline $\begin{array}{l}\text { Blinding of outcome as- } \\
\text { sessors (subjective mea- } \\
\text { sures) }\end{array}$ & Low risk & $\begin{array}{l}\text { Participants were outcome assessors for dryness scores: we did not anticipate } \\
\text { that this would influence the assessment of this outcome measure. }\end{array}$ \\
\hline $\begin{array}{l}\text { Incomplete outcome data } \\
\text { (attrition bias) } \\
\text { All outcomes }\end{array}$ & High risk & $\begin{array}{l}\text { Of } 37 \text { participants randomized, } 7 \text { were excluded from analysis, as they were } \\
\text { unable to complete the study ( } 5 \text { owing to deterioration and } 2 \text { because of rapid } \\
\text { improvement in respiratory status). }\end{array}$ \\
\hline $\begin{array}{l}\text { Selective reporting (re- } \\
\text { porting bias) }\end{array}$ & Unclear risk & $\begin{array}{l}\text { Trial registration not reported in the paper. Unable to establish whether out- } \\
\text { comes were reported according to prepublished protocol or trial registration } \\
\text { documents }\end{array}$ \\
\hline Other bias & Low risk & We identified no other sources of bias. \\
\hline
\end{tabular}

Fernandez 2017

\section{Study characteristics}

\begin{tabular}{ll}
\hline Methods & RCT, parallel-group design. Multicentre study \\
\hline Participants & Total number of randomized participants: 155
\end{tabular}

Participants

Total number of randomized participants: 155

Setting: 4 ICUs; Spain 
Inclusion criteria: adults receiving MV for $>12$ hours and ready for scheduled extubation after a SBT; at high risk for extubation failure

Exclusion criteria: tracheotomy; inability to follow commands; do-not-reintubate order; hypercapnia during SBT

\section{Baseline characteristics:}

Intervention group (HFNC):

- Age, mean (SD): $67.3( \pm 12.1)$ years

- Gender, M/F: 46/32

- $\mathrm{BMI},>30 \mathrm{~kg} / \mathrm{m}^{2}, \mathrm{n}: 14$

- APACHE II, mean (SD) : 21 ( \pm 8.8 )

- $\mathrm{PaCO}_{2}$, mean (SD): not reported

- $\mathrm{PaO}_{2} / \mathrm{FiO}_{2}$, mean (SD): not reported

Control group (conventional oxygen therapy):

- Age, mean (SD): 69.7 ( \pm 13.0 ) years

- Gender, M/F: 55/22

- $\mathrm{BMI},>30 \mathrm{~kg} / \mathrm{m}^{2}, \mathrm{n}: 18$

- APACHE II, mean (SD): 21 ( \pm 8.2 )

- $\mathrm{PaCO}_{2}$, mean (SD): not reported

- $\mathrm{PaO}_{2} / \mathrm{FiO}_{2}$, mean (SD): not reported

- Randomized, $\mathrm{n}=78$; losses, $\mathrm{n}=0$; analysed, $\mathrm{n}=78$

- Details: HFNC via Optiflow, flow initiated at $40 \mathrm{~L} / \mathrm{min}$, humidifier temperature at $37^{\circ} \mathrm{C}$, but switched to noninvasive mode $\left(34^{\circ} \mathrm{C}\right)$ if participant felt excessive warmth. Oxygen titrated to achieve $\mathrm{SpO}_{2}$ at 92 to $95 \%$. After 24 hours, received conventional therapy

Control group:

- Randomized, $\mathrm{n}=77$; losses, $\mathrm{n}=0$; analysed, $\mathrm{n}=77$

- Details: oxygen after extubation via nasal prongs or facemask, regulated by Venturi. Oxygen titrated to achieve $\mathrm{SpO}_{2}$ at 92 to $95 \%$. After 24 hours, continued to receive conventional therapy at discretion of attending team); reintubation; ICU and hospital lengths of stay; hospital mortality

Notes

Funding/declarations of interest: funding not reported. Two authors received conference fees or postdoctoral grant from Fisher \& Paykel Healthcare Ltd.

Study dates: 2013 to 2014

Note: study terminated early owing to low recruitment

\section{Risk of bias}

\begin{tabular}{lll}
\hline Bias & Authors' judgement & Support for judgement \\
\hline $\begin{array}{l}\text { Random sequence genera- } \\
\text { tion (selection bias) }\end{array}$ & Low risk & $\begin{array}{l}\text { Quote: "Randomization was performed via a computerized random-number } \\
\text { table in blocks of four for each hospital”. }\end{array}$ \\
\hline $\begin{array}{l}\text { Allocation concealment } \\
\text { (selection bias) }\end{array}$ & Low risk & Quote: "“allocation was concealed through numbered opaque envelopes". \\
\hline
\end{tabular}


Fernandez 2017 (Continued)

Blinding of participants Unclear risk Blinding was not possible. Although we expected that this would not influence and personnel (performance bias)

All outcomes

\begin{tabular}{|c|c|c|}
\hline $\begin{array}{l}\text { Blinding of outcome as- } \\
\text { sessors (objective out- }\end{array}$ & Low risk & $\begin{array}{l}\text { Blinding of outcome assessors is not described; we did not anticipate that this } \\
\text { would influence the assessment of objective outcome measures }\end{array}$ \\
\hline
\end{tabular}

comes)

Incomplete outcome data Low risk

(attrition bias)

No apparent losses

All outcomes

Selective reporting (re- High risk
porting bias)

Study was prospectively registered with a clinical trials register

(NCT01820507). We noted that some outcomes were reported in the published report but not listed in the trials register documents (hospital and ICU lengths of stay); this could indicate risk of selective reporting bias for these outcomes.

\begin{tabular}{ll}
\hline Other bias $\quad$ Low risk $\quad$ We identified no other sources of bias. \\
\hline
\end{tabular}

\section{Frat 2015}

\section{Study characteristics}

\begin{tabular}{ll}
\hline Methods & RCT, parallel-group design. Multicentre study \\
\hline
\end{tabular}

Participants

\section{Total number of participants: 313}

Setting: 23 ICUs; France and Belgium

Inclusion criteria: consecutive patients, aged $\geq 18$ years, respiratory rate $>25$ breaths per minute, $\mathrm{PaO}_{2} / \mathrm{FiO}_{2} \leq 300 \mathrm{mmHg}$ while patient was breathing oxygen at flow rate $\geq 10 \mathrm{~L} / \mathrm{min}$ for at least $15 \mathrm{~min}$ utes, $\mathrm{PaCO}_{2}$ not higher than $45 \mathrm{mmHg}$, absence of clinical history of underlying chronic respiratory failure

Exclusion criteria: $\mathrm{PaCO}_{2}>45 \mathrm{mmHg}$, exacerbation of asthma or chronic respiratory failure, cardiogenic pulmonary oedema, severe neutropenia, haemodynamic instability, use of vasopressors, GCS $\leq$ 12 , contraindications to NIV, urgent need for tracheal intubation, a do-not-resuscitate order, or decision to not participate

\section{Baseline characteristics:}

Intervention group (HFNC):

- Age mean (SD): $61( \pm 16)$ years

- Gender, M/F: 75/31

- BMI, mean (SD): $25( \pm 5)$

- SAPS II:, mean (SD): 25 ( \pm 9$)$

- $\mathrm{PaCO}_{2}$ mean (SD): $36( \pm 6) \mathrm{mmHg}$

- $\mathrm{PaO}_{2} / \mathrm{FiO}_{2}$ mean (SD): $157( \pm 89) \mathrm{mmHg}$

- Respiratory rate mean (SD): 33 ( \pm 6 ) breaths/min

Control group 1 (standard oxygen therapy):

- Age mean (SD): $59( \pm 17)$ years

- Gender, M/F: 63\31 
Frat 2015 (Continued)

- BMI, mean (SD): $26( \pm 5)$

- SAPS II:, mean (SD): $24( \pm 9)$

- $\mathrm{PaCO}_{2}$ mean (SD): $35( \pm 5) \mathrm{mmHg}$

- $\mathrm{PaO}_{2} / \mathrm{FiO}_{2}$ mean (SD): $161( \pm 73) \mathrm{mmHg}$

- Respiratory rate mean (SD): 32 ( \pm 6 ) breaths/min

Control group 2 (non-invasive ventilation):

- Age mean (SD): $61( \pm 17)$ years

- Gender, M/F: 74/36

- BMI, mean (SD): $26( \pm 6)$

- SAPS II:, mean (SD): $27( \pm 9)$

- $\mathrm{PaCO}_{2}$ mean (SD): $34( \pm 6) \mathrm{mmHg}$

- $\mathrm{PaO}_{2} / \mathrm{FiO}_{2}$ mean (SD): $149( \pm 72) \mathrm{mmHg}$

- Respiratory rate mean (SD): 33 ( \pm 7 ) breaths/min

Interventions

Intervention group (HFNC):

- Randomized, $\mathrm{n}=106$; losses, $\mathrm{n}=0$; analysed, $\mathrm{n}=106$

- Details: Oxygen passed through heated humidifier, applied continuously through large-bore nasal prongs; gas flow rate $50 \mathrm{~L} / \mathrm{min}, \mathrm{FiO}_{2} 1.0$ at initiation (Optiflow); adjusted to maintain $\mathrm{SpO}_{2} \geq 92 \%$; for at least 2 calendar days, then this could be stopped or participant switched to standard oxygen therapy

Control group (standard-oxygen therapy):

- Randomized, $\mathrm{n}=9$; losses, $\mathrm{n}=2$ (withdrew consent); analysed, $\mathrm{n}=94$

- Details: Continuously through non-rebreather face mask; flow rate $\geq 10 \mathrm{~L} / \mathrm{min}$; adjusted to maintain $\mathrm{SpO}_{2} \geq 92 \%$; until participant recovered or was intubated

Control group (non-invasive ventilation):

- Randomized, $n=111$, losses, $n=1$; analysed, $n=110$

- Details: Through a face mask connected to an ICU ventilator with pressure support applied in NIV mode; adjusted to obtain expired tidal volume of 7 to $10 \mathrm{~mL} / \mathrm{kg}$ of predicted body weight, with initial PEEP between 2 and $10 \mathrm{~cm}$ of water; adjusted to maintain $\mathrm{SpO}_{2} \geq 92 \%$; minimum of 8 hours per day for at least 2 calendar days; applied during sessions of at least 1 hour, could be resumed if respiratory rate $>25$ breaths per minute or $\mathrm{SpO}_{2}$ less than $92 \%$; between non-invasive ventilation sessions, participants received high-flow oxygen

Outcomes

Participants requiring endotracheal intubation within 28 days of randomization, mortality in ICU, mortality at 90 days, number of ventilator-free days between day 1 and day 28, duration of ICU stay, complications during ICU stay, dyspnoea, comfort

Notes

Funding/declarations of interest: equipment provided by Fisher \& Paykel Healthcare Ltd, but manufacturer had no involvement in the study.

Study dates: February 2011 to April 2013

Note: we noted an unequal number of participants in each group which we could not explain. An appropriate method of randomization was described, and baseline characteristics were largely comparable.

\section{Risk of bias}

\begin{tabular}{lll}
\hline Bias & Authors' judgement & Support for judgement \\
\hline $\begin{array}{l}\text { Random sequence genera- } \\
\text { tion (selection bias) }\end{array}$ & Low risk & $\begin{array}{l}\text { Use of centralized Web-based management system, blocks of 6, stratified by } \\
\text { centre and history or no history of cardiac insufficiency }\end{array}$ \\
\hline \hline
\end{tabular}

High-flow nasal cannulae for respiratory support in adult intensive care patients (Review) 
Frat 2015 (Continued)

\begin{tabular}{l}
$\begin{array}{l}\text { Allocation concealment } \quad \text { Unclear risk } \\
\text { (selection bias) }\end{array}$ \\
\hline
\end{tabular}

\begin{tabular}{|c|c|c|}
\hline $\begin{array}{l}\text { Blinding of participants } \\
\text { and personnel (perfor- }\end{array}$ & Unclear risk & $\begin{array}{l}\text { Blinding was not possible. Although we expected that this would not influence } \\
\text { outcome data, we could not be certain of this. }\end{array}$ \\
\hline
\end{tabular}
mance bias)

All outcomes

\begin{tabular}{|c|c|c|}
\hline $\begin{array}{l}\text { Blinding of outcome as- } \\
\text { sessors (objective out- }\end{array}$ & Low risk & $\begin{array}{l}\text { Blinding of outcome assessors was not described; we did not anticipate that } \\
\text { this would influence the assessment of objective outcome measures. }\end{array}$ \\
\hline
\end{tabular}
comes)

\begin{tabular}{|c|c|c|}
\hline $\begin{array}{l}\text { Blinding of outcome as- } \\
\text { sessors (subjective mea- } \\
\text { sures) }\end{array}$ & Low risk & $\begin{array}{l}\text { Participants were outcome assessors for dyspnoea and comfort: we did not } \\
\text { anticipate that this would influence the assessment of these outcome mea- } \\
\text { sures. }\end{array}$ \\
\hline
\end{tabular}

Incomplete outcome data Low risk (attrition bias)

All outcomes

Selective reporting (re- High risk
porting bias)

porting bias)

\begin{abstract}
3 losses ( 2 in standard oxygen group and 1 in NIV group) due to withdrawal of consent. Small number of losses unlikely to influence outcome data
\end{abstract}

NCT01320384. Study prospectively registered. We noted that most outcomes were reported in the published paper or its supplementary appendix. However, study authors described an outcome as the use of NIV as an escalation therapy in the HFNC or standard oxygen therapy groups, but did not report data for this outcome; we judged selective reporting bias to be high because this was a primary outcome in our first comparison group.

Other bias High risk Participants in NIV monitoring group were given HFNC between ventilation
sessions.

\section{Futier 2016}

\section{Study characteristics}

\begin{tabular}{ll}
\hline Methods & RCT, parallel-group design. Multicentre study \\
\hline
\end{tabular}

Participants

Number of randomized participants: 228

Setting: 3 ICUs; France

Inclusion criteria: adults scheduled for planned or unplanned abdominal, or abdominal and thoracic surgery with anticipated duration of $\geq 2$ hours, and moderate to high risk of postoperative pulmonary complications

Exclusion criteria: lack of informed consent; BMI > $35 \mathrm{~kg} / \mathrm{m}^{2}$; life-threatening condition requiring emergency surgery; obstructive sleep apnoea syndrome, pregnant

\section{Baseline characteristics:}

Intervention group (HFNC):

- Age, mean (SD): $62( \pm 12)$ years

- Gender, M/F: 61/47

- BMI, mean (SD): $25( \pm 4) \mathrm{kg} / \mathrm{m}^{2}$

- ASA I/II/ $\geq$ III, n: 20/72/7

- $\mathrm{PaCO}_{2}$, mean (SD): not reported 
Futier 2016 (Continued)

- $\mathrm{PaO}_{2} / \mathrm{FiO}_{2}$, mean (SD): not reported

Control group (standard oxygen therapy):

- Age, mean (SD): $61( \pm 13)$ years

- Gender, M/F: 64/48

- BMI, mean (SD): $25( \pm 4) \mathrm{kg} / \mathrm{m}^{2}$

- ASA I/II/ $\geq$ III, n: 20/75/17

- $\mathrm{PaCO}_{2}$, mean (SD): not reported

- $\mathrm{PaO}_{2} / \mathrm{FiO}_{2}$, mean (SD): not reported

Interventions

Intervention group (HFNC):

- Randomized, $\mathrm{n}=108$; losses, $\mathrm{n}=0$; analysed, $\mathrm{n}=108$

- Details: HFNC using Optiflow, was delivered continuously at a flow rate of 50 to $60 \mathrm{~L} / \mathrm{min}$, starting after tracheal extubation at the end of surgery until the morning of postoperative day 1 . Titrated to maintain $\mathrm{SpO}_{2}$ of $\geq 95 \%$

Control group (standard oxygen therapy):

- Randomized, $n=112$; losses, $n=0$; analysed, $n=112$

- Details: standard oxygen therapy, using nasal prongs or facemask, was delivered continuously, starting after tracheal extubation at the end of surgery until the morning of postoperative day 1 . Titrated to maintain $\mathrm{SpO}_{2}$ of $\geq 95 \%$.

Note: study authors reported that 220 participants were randomized, and that 8 of these were excluded ( 3 because surgery was $<2$ hours, and 5 because of other protocol violations), then a further 8 participants were recruited. Study authors did not report to which group the 8 lost participants belonged.

Outcomes

Hypoxaemia, postoperative pulmonary complications within 7 days after surgery, need for additional oxygen therapy at end of treatment; development of postoperative hypoxaemia, pneumonia, reintubation and/or use of curative NIV because of postoperative respiratory failure; postoperative gas exchange after discontinuation of allocated treatment; respiratory comfort (numerical rating scale from 0 to 10); duration of hospital and ICU stay, in-hospital mortality

Notes

Funding/declarations of interest: funding for study was not reported. Some individual authors received consulting fees, lecture fees, nonfinancial support, travel expenses, or research grants from one or more of: Fresenius Kabi, General Electrics Healthcare, Drager, Fisher \& Paykel Healthcare Ltd, Merck Sharp \& Dohme, Baxter Gambro, Astellas, LFB Bio-medicaments, and Pfizer. Study authors declared no competing interests.

Study dates: 6 November 2013 to 1 March 2015

\section{Risk of bias}

\begin{tabular}{|c|c|c|}
\hline Bias & Authors' judgement & Support for judgement \\
\hline $\begin{array}{l}\text { Random sequence genera- } \\
\text { tion (selection bias) }\end{array}$ & Low risk & Computer-generated randomization \\
\hline $\begin{array}{l}\text { Allocation concealment } \\
\text { (selection bias) }\end{array}$ & Low risk & Allocation managed externally by a centralised telephone system \\
\hline $\begin{array}{l}\text { Blinding of participants } \\
\text { and personnel (perfor- } \\
\text { mance bias) } \\
\text { All outcomes }\end{array}$ & Unclear risk & $\begin{array}{l}\text { Blinding was not possible. Although we expected that this would not influence } \\
\text { outcome data, we could not be certain of this. }\end{array}$ \\
\hline
\end{tabular}


Futier 2016 (Continued)

Blinding of outcome as- Low risk Treatment allocation was concealed to outcome assessors. sessors (objective outcomes)

\begin{tabular}{|c|c|c|}
\hline $\begin{array}{l}\text { Blinding of outcome as- } \\
\text { sessors (subjective mea- } \\
\text { sures) }\end{array}$ & Low risk & $\begin{array}{l}\text { Treatment allocation was concealed to outcome assessors. Participants were } \\
\text { outcome assessors for respiratory comfort: we did not anticipate that this } \\
\text { would influence the assessment of this outcome measure. }\end{array}$ \\
\hline $\begin{array}{l}\text { Incomplete outcome data } \\
\text { (attrition bias) } \\
\text { All outcomes }\end{array}$ & Low risk & Only small number of losses (8 participants) \\
\hline
\end{tabular}

\begin{tabular}{ll}
\hline $\begin{array}{l}\text { Selective reporting (re- } \\
\text { porting bias) }\end{array}$ & Low risk \\
& $\begin{array}{l}\text { Study was prospectively registered with a clinical trials register } \\
\text { (NCT01887015). Outcomes were reported according to those described in clini- } \\
\text { cal trials register. }\end{array}$
\end{tabular}

Other bias Low risk We identified no other sources of bias.

Grieco 2020

\section{Study characteristics}

\begin{tabular}{|c|c|}
\hline Methods & RCT, cross-over design. Single-centre study \\
\hline Participants & 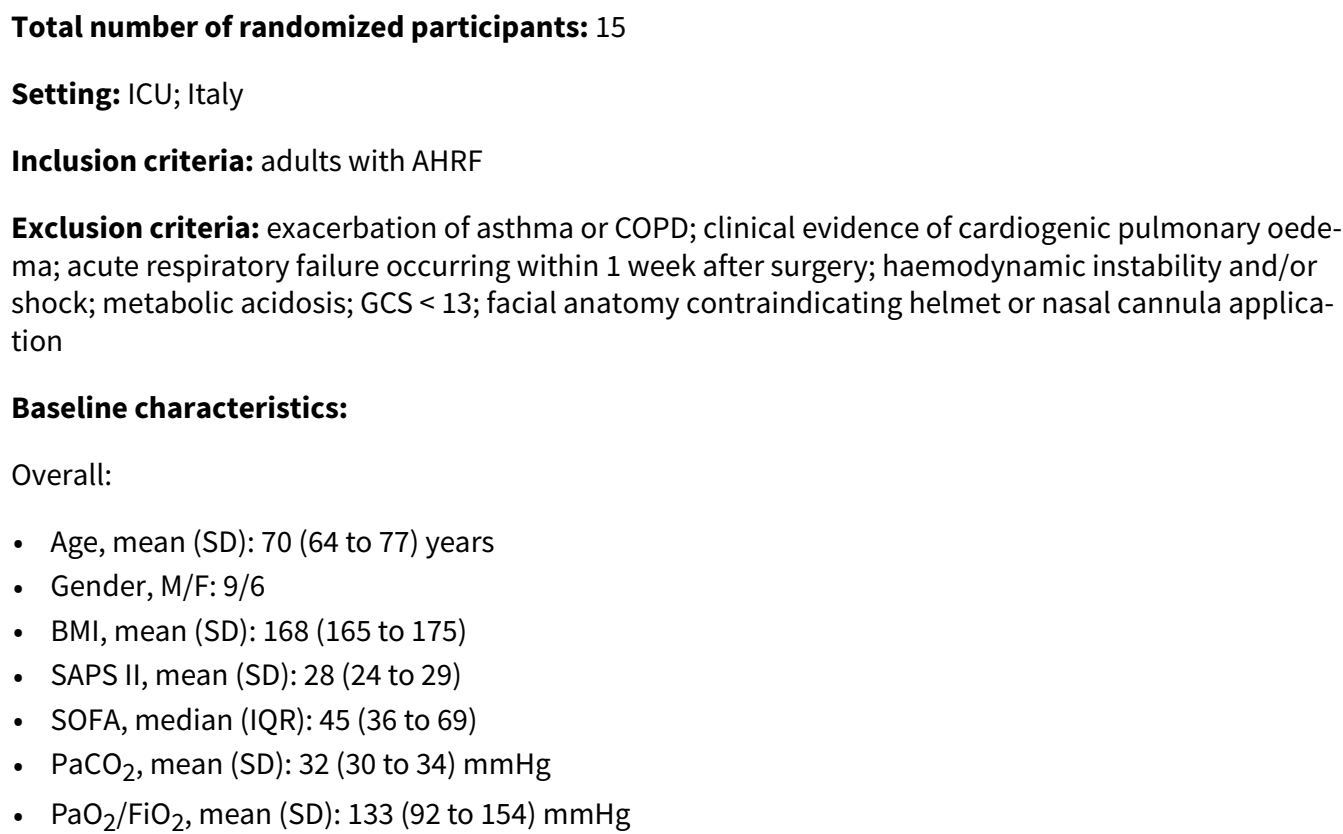 \\
\hline
\end{tabular}

Interventions

Cross-over study, with each phase lasting 60 minutes

- HFNC: using $\mathrm{AIRVO}_{2}$ device, Fisher \& Paykel Healthcare, with a heated humidifier (MR860). Gas flow set at $50 \mathrm{~L} / \mathrm{min}$, and humidifier temperature at $37^{\circ} \mathrm{C}$. $\mathrm{FiO}_{2}$ titrated to obtain $\mathrm{SpO}_{2} \geq 92 \%$ and $\leq 98 \%$

- Helmet NIV: through bi-tube circuit with no humidification. Initial pressure support was 8 to $10 \mathrm{~cm}$ $\mathrm{H}_{2} \mathrm{O}$, adjusted for peak inspiratory flow of 100 to $150 \mathrm{~L} / \mathrm{min}$, up to a maximum of $20 \mathrm{~cm} \mathrm{H}_{2} \mathrm{O}$; PEEP at 10 to $12 \mathrm{~cm} \mathrm{H} \mathrm{H}_{2} \mathrm{O}$; flow trigger was $2 \mathrm{~L} / \mathrm{min}$ and increased in presence of auto-triggering. $\mathrm{FiO}_{2}$ titrated to obtain $\mathrm{SpO}_{2} \geq 92 \%$ and $\leq 98 \%$ 
Grieco 2020 (Continued)

Between interventions, there was a 15-minute washout period with heated and humidified (MR860; Fisher \& Paykel Healthcare) oxygen therapy at a flow rate of $50 \mathrm{~L} / \mathrm{min}$ via a non-rebreathing face mask (temperature of the humidification chamber set at $37^{\circ} \mathrm{C}, \mathrm{FiO}_{2}$ set to achieve a $\mathrm{SpO}_{2}>92 \%$ and < $98 \%$ )

Outcomes $\mathrm{PaO}_{2} / \mathrm{FiO}_{2} ; \mathrm{PaCO}_{2}$; respiratory rate; inspiratory effort; work of breathing; comfort; dyspnoea; end-inspiratory and end-expiratory transpulmonary pressure

Note: we did not include outcome data in the review because the study authors did not report outcome data from the first study period.

Notes

Funding/declarations of interest: research grant from Società Italiana di Anestesia Analgesia Rianimazione e Terapia Intensiva; and Merck Sharp and Dohme

Study dates: May 2017 and December 2018

\section{Study characteristics}

\begin{tabular}{|c|c|}
\hline Methods & $\mathrm{RCT}$, parallel-group design. Multicentre study \\
\hline Participants & 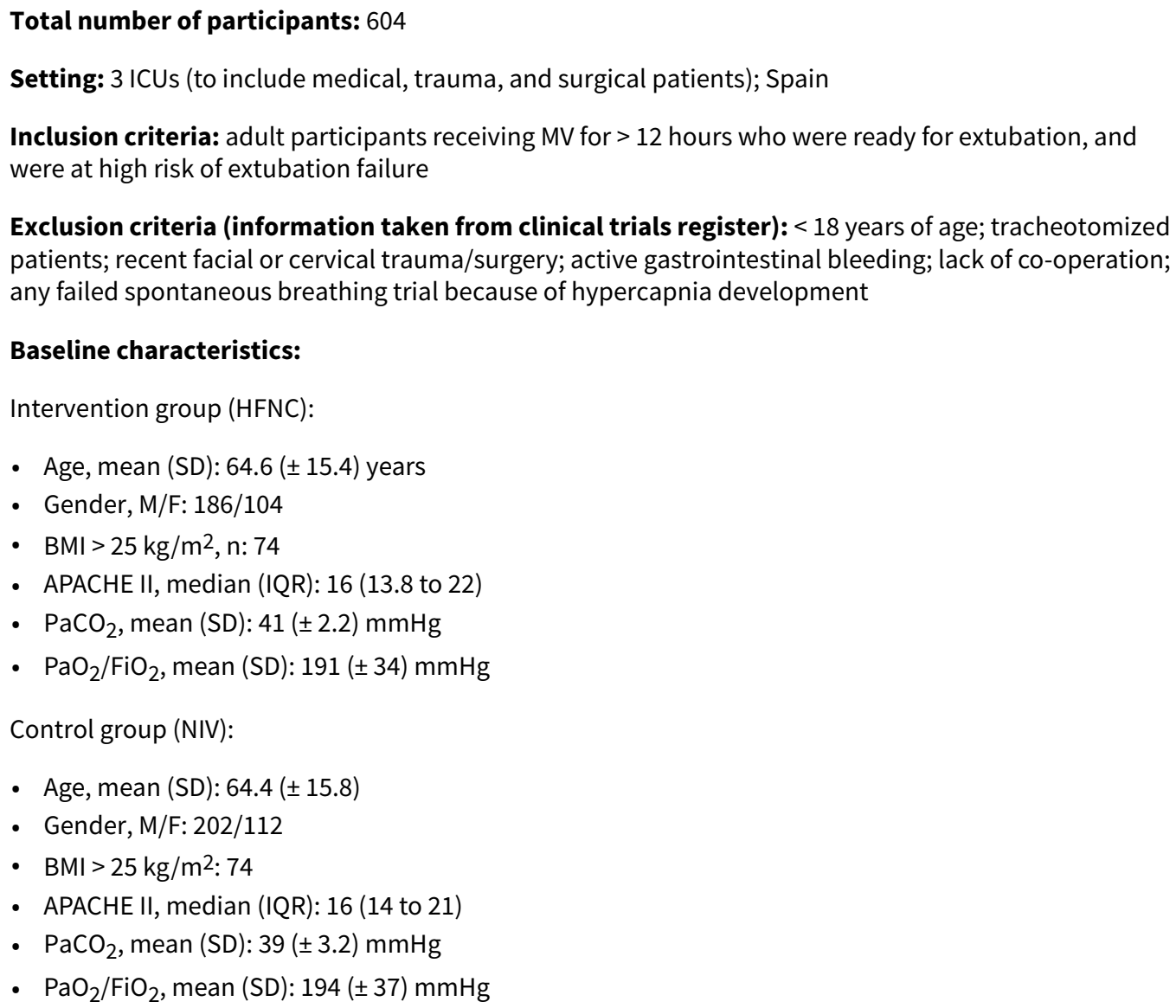 \\
\hline
\end{tabular}

Interventions

Intervention group (HFNC):

- Randomized, $\mathrm{n}=290$; losses, $\mathrm{n}=2$ (discontinued study or loss to follow-up); analysed, $\mathrm{n}=288$ (study authors also calculated ITT analysis) 
- Details: Optiflow high-flow oxygen immediately after extubation through specific nasal cannulae; flow initially set at $10 \mathrm{~L} / \mathrm{min}$ and titrated upwards in $5 \mathrm{~L} /$ min steps. $\mathrm{FiO}_{2}$ regularly adjusted to target $\mathrm{SpO}_{2}>$ $92 \%$. After 24 hours, high flow was stopped and, if necessary, participants received standard oxygen therapy.

Control group (NIV):

- Randomized, $n=314$; losses, $n=2$ (discontinued study or loss to follow-up); analysed, $n=312$ (study authors also calculated ITT analysis)

- Details: full face mask with BiPAP Vision immediately after extubation for 24 hours. Then NIV was withdrawn and oxygen was given via Venturi mask. PEEP and inspiratory pressure support adjusted to target respiratory rate of 25 breaths/min and adequate gas exchange. Sedatives to increase tolerance to NIV were not allowed.

Outcomes

Reintubation within 72 hours; post-extubation respiratory failure; respiratory infection, sepsis, multiple organ failure, ICU and hospital length of stay, ICU and hospital mortality; adverse events; time to reintubation; duration of respiratory support; respiratory effects $\left(\mathrm{PaO}_{2} / \mathrm{FiO}_{2}\right.$ and $\left.\mathrm{PaCO}_{2}\right)$

Note: we did not report data for physiological variables $\left(\mathrm{PaO}_{2} / \mathrm{FiO}_{2}\right.$ and $\left.\mathrm{PaCO}_{2}\right)$ because these were reported only for participants who were reintubated or had post-extubation respiratory failure. We did not include data for duration of respiratory support because these data were reported only for the NIV group.

Notes

Funding sources/declarations of interest: study received no external funding. One author declared travel expenses from Fisher and Paykel Healthcare Ltd; no other disclosures reported

Study dates: September 2012 to October 2014

Note: Hernandez 2016a and Hernandez 2016b were registered as one study in the clinical trials register.

\section{Risk of bias}

\begin{tabular}{lll}
\hline Bias & Authors' judgement & Support for judgement \\
\hline $\begin{array}{l}\text { Random sequence genera- } \\
\text { tion (selection bias) }\end{array}$ & Low risk & Use of random number generator \\
\hline $\begin{array}{l}\text { Allocation concealment } \\
\text { (selection bias) }\end{array}$ & Low risk & $\begin{array}{l}\text { Allocation concealed through use of telephone call centre that generated ran- } \\
\text { domization }\end{array}$ \\
\hline $\begin{array}{l}\text { Blinding of participants } \\
\begin{array}{l}\text { and personnel (perfor- } \\
\text { mance bias) } \\
\text { All outcomes }\end{array}\end{array}$ & Unclear risk & $\begin{array}{l}\text { Blinding was not possible. Although we expected that this would not influence } \\
\text { outcome data, we could not be certain of this. }\end{array}$ \\
\hline
\end{tabular}

\begin{tabular}{|c|c|c|}
\hline $\begin{array}{l}\text { Blinding of outcome as- } \\
\text { sessors (objective out- } \\
\text { comes) }\end{array}$ & Low risk & $\begin{array}{l}\text { Blinding of outcome assessors was not described; we did not anticipate that } \\
\text { this would influence the assessment of objective outcome measures. }\end{array}$ \\
\hline
\end{tabular}

\begin{tabular}{lll}
\hline $\begin{array}{l}\text { Incomplete outcome data } \\
\text { (attrition bias) } \\
\text { All outcomes }\end{array}$ & Low risk & Only two participants in each group were lost to follow-up. \\
\hline $\begin{array}{l}\text { Selective reporting (re- } \\
\text { porting bias) }\end{array}$ & Low risk & $\begin{array}{l}\text { Study was prospectively registered with a clinical trials register } \\
\text { (NCT01191489). Outcomes were reported as described in the prospective reg- } \\
\text { istration documents. }\end{array}$
\end{tabular}

Other bias Low risk We identified no other sources of bias.




\section{Study characteristics}

\begin{tabular}{|c|c|}
\hline Methods & RCT, parallel-group design. Multicentre study \\
\hline Participants & 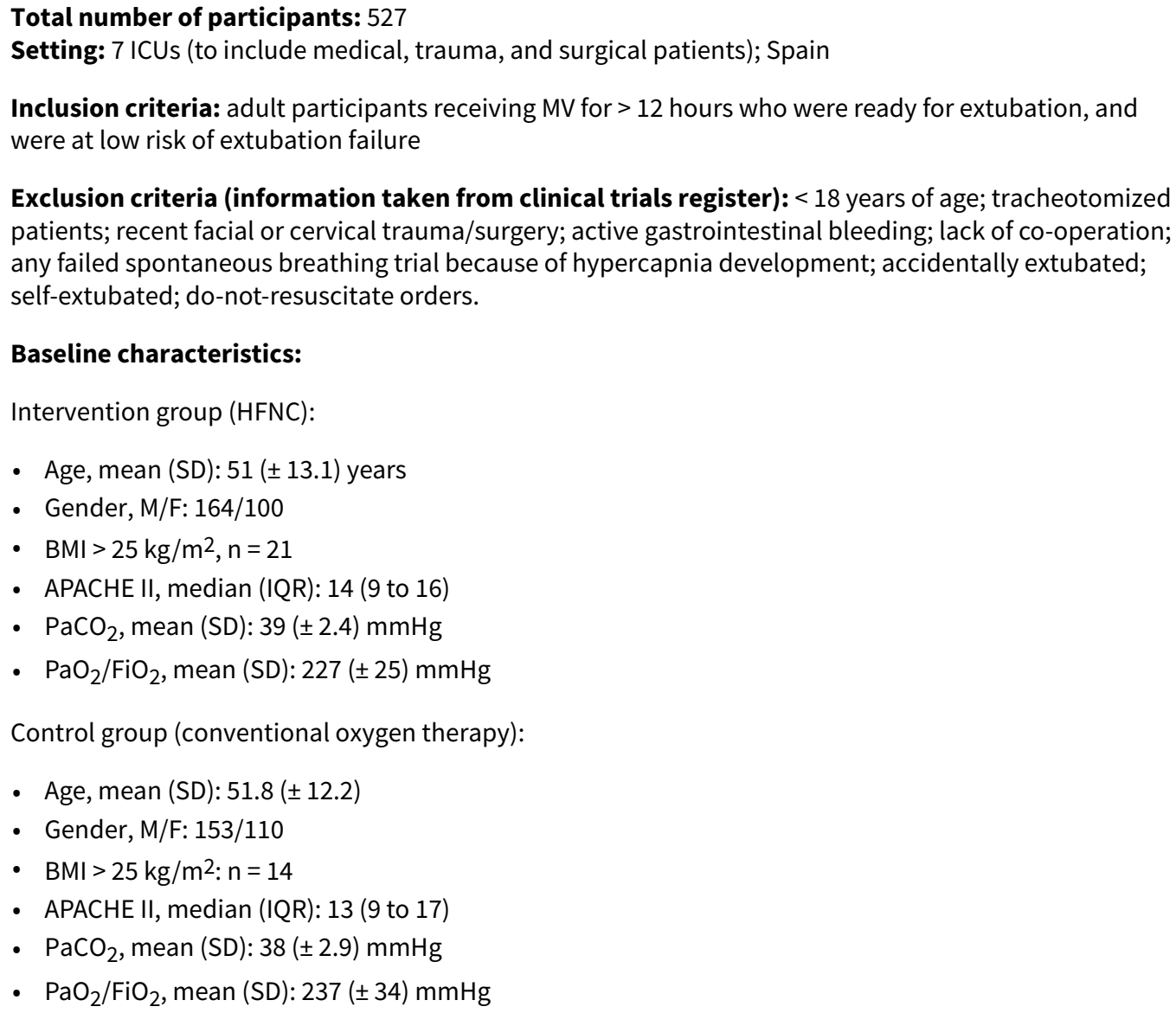 \\
\hline
\end{tabular}

- Randomized, $\mathrm{n}=264$; losses, $\mathrm{n}=0$; analysed, $\mathrm{n}=264$

- Details: Optiflow high-flow oxygen immediately after extubation through specific nasal cannulae; flow initially set at $10 \mathrm{~L} / \mathrm{min}$ and titrated upwards in $5 \mathrm{~L} /$ min steps. $\mathrm{FiO}_{2}$ regularly adjusted to target $\mathrm{SpO}_{2}>$ $92 \%$. After 24 hours, high flow was stopped, and if necessary, participants received standard oxygen therapy.

Control group (standard oxygen therapy):

- Randomized, $\mathrm{n}=263$; losses, $\mathrm{n}=0$; analysed, $\mathrm{n}=263$

- Details: conventional oxygen therapy applied continuously through nasal cannula or non-rebreather facemask immediately after extubation for 24 hours. Oxygen flow adjusted to target $\mathrm{SpO}_{2}>92 \%$.

Outcomes

Re-intubation within 72 hours, post-extubation respiratory failure, respiratory infection, sepsis and multi-organ failure, ICU and hospital length of stay, ICU and hospital mortality, adverse events, time to reintubation, respiratory effects $\left(\mathrm{PaO}_{2} / \mathrm{FiO}_{2}\right.$ and $\left.\mathrm{PaCO}_{2}\right)$, adverse events (nasal mucosa or skin trauma)

Note: we did not report data for physiological variables $\left(\mathrm{PaO}_{2} / \mathrm{FiO}_{2}\right.$ and $\left.\mathrm{PaCO}_{2}\right)$ because these were reported only for participants who were reintubated or had post-extubation respiratory failure. 
Hernandez 2016b (Continued)

Notes
Funding/declarations of interest: no external funding. Fisher and Paykel Healthcare Ltd supplied airoxygen blenders to 2 of the ICUs; Fisher and Paykel had no involvement in study design and conduct, or in preparation of final manuscript etc.

Study dates: September 2012 to October 2014

Note: Hernandez 2016a and Hernandez 2016b were registered as one study in the clinical trials register.

\section{Risk of bias}

\begin{tabular}{lll}
\hline Bias & Authors' judgement & Support for judgement \\
\hline $\begin{array}{l}\text { Random sequence genera- } \\
\text { tion (selection bias) }\end{array}$ & Low risk & Use of random number generator \\
\hline $\begin{array}{l}\text { Allocation concealment } \\
\text { (selection bias) }\end{array}$ & Low risk & $\begin{array}{l}\text { Allocation concealed through use of telephone call centre that generated ran- } \\
\text { domization }\end{array}$ \\
\hline $\begin{array}{l}\text { Blinding of participants } \\
\text { and personnel (perfor- } \\
\text { mance bias) }\end{array}$ & Unclear risk & $\begin{array}{l}\text { Blinding was not possible. Although we expected that this would not influence } \\
\text { All outcomes }\end{array}$ \\
\hline $\begin{array}{l}\text { Blinding of outcome as- } \\
\text { sessors (objective out- } \\
\text { comes) }\end{array}$ & Low risk & $\begin{array}{l}\text { Blinding of outcome assessors was not described; we did not anticipate that } \\
\text { this would influence the assessment of objective outcome measures. }\end{array}$ \\
\hline $\begin{array}{l}\text { Incomplete outcome data } \\
\text { (attrition bias) } \\
\begin{array}{l}\text { All outcomes } \\
\hline\end{array}\end{array}$ & Low risk & No losses \\
\hline
\end{tabular}

Study prospectively registered with a clinical trials register (NCT01191489). Outcomes were reported as described in the prospective registration documents.

Other bias Low risk We identified no other sources of bias.

Hu 2020

\section{Study characteristics}

\begin{tabular}{ll}
\hline Methods & RCT, parallel-group design. Single-centre study \\
\hline
\end{tabular}

\section{Participants}

\section{Total number of randomized participants: 56}

Setting: medical ICU; Taiwan

Inclusion criteria: adults admitted to the ICU with acute respiratory failure and mechanically ventilated for $>48$ hours, successfully passed a SBT; meeting at least one risk factor for high-risk extubation failure

Exclusion criteria: < 20 years of age; tracheostomy; pregnancy; facial trauma with intolerable post-extubation facial mask or HFNC use; acute gastrointestinal bleeding; planning to use NIV after extubation

\section{Baseline characteristics:}

Intervention group (HFNC): 
Hu 2020 (Continued)

- Age, mean (SD): $72.9( \pm 13.1)$ years

- Gender, M/F: 22/7

- BMI, > $30 \mathrm{~kg} / \mathrm{m}^{2}, \mathrm{n}: 3$

- APACHE II, median IQR: 27 (23 to 29)

- $\mathrm{PaCO}_{2}$, mean (SD): $42( \pm 7.9) \mathrm{mmHg}$

- $\mathrm{PaO}_{2} / \mathrm{FiO}_{2}$, mean (SD): $320( \pm 89.6) \mathrm{mmHg}$

Control group (conventional oxygen therapy):

- Age, mean (SD): $74.9( \pm 11.4)$ years

- Gender, M/F: 17/10

- $\mathrm{BMI},>30 \mathrm{~kg} / \mathrm{m}^{2}, \mathrm{n}: 3$

- APACHE II, median (IQR): 25 (22-30)

- $\mathrm{PaCO}_{2}$, mean (SD): $38( \pm 7.4) \mathrm{mmHg}$

- $\mathrm{PaO}_{2} / \mathrm{FiO}_{2}$, mean (SD): $279( \pm 90.6) \mathrm{mmHg}$

Interventions Intervention group:

- Randomized, $\mathrm{n}=29$; losses, $\mathrm{n}=0$; analysed, $\mathrm{n}=29$

- Details: HFNC using Optiflow, immediately after extubation, humidified temperature initially set to 37 ${ }^{\circ} \mathrm{C}$, flow rate initially at $40 \mathrm{~L} / \mathrm{min}$ with adjustments of 5 to $10 \mathrm{~L} / \mathrm{min}$ (to maximum of $60 \mathrm{~L} / \mathrm{min}$ ). $\mathrm{FiO}_{2}$ titrated to maintain $\mathrm{SpO}_{2}>92 \%$ (or $88-95 \%$ for compensated hypercapnia). Applied for at least 72 hours, then maintained or given conventional therapy

Control group:

- Randomized, $\mathrm{n}=27$; losses, $\mathrm{n}=0$; analysed, $\mathrm{n}=27$

- Details: post-extubation, conventional oxygen therapy delivered continuously through nasal prongs with flow rate 1 to $4 \mathrm{~L} / \mathrm{min}$, or via Venturi facemask with oxygen and flow titrated to deliver $\mathrm{FiO}_{2}$ between $35 \%$ and $100 \%$ and to maintain $\mathrm{SpO}_{2}>92 \%$ (or 88 to $95 \%$ for compensated hypercapnia). Applied for at least 72 hours, then maintained if required

Outcomes

Respiratory failure within 72 hours (requiring rescue management with oxygen therapy, NIV, or reintubation); time to post-extubation failure within 72 hours; multiple organ failure; ICU length of stay; 28 day all-cause mortality (in-hospital); 48-hour respiratory and haemodynamic variables (heart rate, MAP, $\mathrm{PaO}_{2}, \mathrm{PaCO}_{2}$ ); causes of respiratory failure (dyspnoea or hypoxia, respiratory acidosis, decreased levels of consciousness, stridor or upper airway problems)

Notes

Funding/declarations of interest: funding not reported. Study authors declared no competing interests.

Study dates: September 2014 to December 2016

\section{Risk of bias}

\section{Bias}

Random sequence genera- Low risk tion (selection bias)

\section{Authors' judgement Support for judgement}

1:1 block randomization used

\begin{tabular}{lll}
\hline $\begin{array}{l}\text { Allocation concealment } \\
\text { (selection bias) }\end{array}$ & Unclear risk & No details
\end{tabular}

Blinding of participants

Unclear risk

Blinding was not possible and we did not expect it to influence outcome data. and personnel (perforHowever, the influence on RoB remains unclear.

mance bias)

All outcomes 
Hu 2020 (Continued)

Blinding of outcome as- Low risk Blinding of outcome assessors. Although we expected that this would not insessors (objective outfluence outcome data, we could not be certain of this. comes)

\begin{tabular}{lll}
$\begin{array}{l}\text { Incomplete outcome data } \\
\text { (attrition bias) } \\
\text { All outcomes }\end{array}$ & Low risk \\
\hline $\begin{array}{l}\text { Selective reporting (re- } \\
\text { porting bias) }\end{array}$ & High risk & $\begin{array}{l}\text { Study was prospectively registered with a clinical trials register (NCT } \\
\text { 02290548). Whilst most review outcomes were reported according to these } \\
\text { trial register documents, we noted that study authors did not report data for } \\
\text { hospital length of stay and for pneumonia; this may indicate selective report- } \\
\text { ing bias for these outcomes. }\end{array}$
\end{tabular}

Other bias Low risk We identified no other sources of bias.

Jing 2019

\section{Study characteristics}

\begin{tabular}{ll}
\hline Methods & RCT, parallel-group design. Single-centre study \\
\hline Participants & Total number of randomized participants: 42 \\
& Setting: ICU; China \\
& Inclusion criteria: people with COPD who were intubated for exacerbation, with hypercapnia $\left(P a C O_{2}>\right.$ \\
& $45 \mathrm{mmHg})$ at time of extubation, met the 'pulmonary infection control window' criteria \\
& Exclusion criteria: tracheotomy; severe dysfunction of other organs; haemodynamic instability; facial \\
injury, burns, or deformities; unco-operative; copious secretions with weak cough ability; gastric over- \\
distention, vomiting; untreated pneumothorax; rhinitis, nasal congestion, deformities or blockage; re- \\
fusal to participate in the study
\end{tabular}

\section{Baseline characteristics:}

Intervention group (HFNC):

- Age, mean (SD): $77.4( \pm 6.8)$ years

- Gender, M/F: not reported

- BMI, mean (SD): not reported

- APACHE II, mean (SD): $11.8( \pm 3.1)$

- $\mathrm{PaCO}_{2}$, mean (SD): $53.2( \pm 6.7) \mathrm{mmHg}$

- $\mathrm{PaO}_{2} / \mathrm{FiO}_{2}$, mean (SD): $239.2( \pm 80.8) \mathrm{mmHg}$

- Respiratory rate, mean (SD): 18.3 (3.5) breaths/min

Control group (NIV):

- Age, mean (SD): $73.9( \pm 6.9)$ years

- Gender, M/F: not reported

- BMI, mean (SD): not reported

- APACHE II, mean (SD): $10.4( \pm 2.5)$

- $\mathrm{PaCO}_{2}$, mean (SD): $53.7( \pm 8.6) \mathrm{mmHg}$

- $\mathrm{PaO}_{2} / \mathrm{FiO}_{2}$, mean (SD): $250.8( \pm 75.8) \mathrm{mmHg}$

- Respiratory rate, mean (SD): 19.2 (4.1) breaths/min 
Jing 2019 (Continued)

Interventions
Intervention group (HFNC):

- Randomized, $n=22$; losses, $n=2$ ( 1 did not receive intervention; 1 discontinued after 25 hours because of transfer to another hospital); analysed for failure requiring NIV or reintubation, mortality, comfort score, and ICU length of stay, $\mathrm{n}=22$ (use of ITT analysis); analysed for respiratory rate, $\mathrm{PaCO}_{2}, \mathrm{PaO}_{2}$ / $\mathrm{FiO}_{2}, \mathrm{n}=20$ (per protocol analysis)

- Details: HFNC using either Optiflow or $\mathrm{AIRVO}_{2}$, nasal cannulae chosen according to participants' nostrils. Humidifier temperature set to $37^{\circ} \mathrm{C}, \mathrm{FiO}_{2}$ adjusted to maintain $\mathrm{SpO}_{2}$ at 88 to $92 \%$. In the 48 hours post-extubation, HFNC was used at least 8 hours/day.

Control group (NIV):

- Randomized, $n=20 ;$ losses, $n=0$; analysed, $n=20$

- Details: NIV using VPAP III ST, ResMed USA, with standard oral-nasal mask. Inspiratory positive airway pressure initiated at 10 to $12 \mathrm{~cm} \mathrm{H} \mathrm{O}$, and expiratory positive airway pressure started at 4 to $5 \mathrm{~cm}$ $\mathrm{H}_{2} \mathrm{O}$. Oxygen adjusted to maintain $\mathrm{SpO}_{2}$ at 88 to $92 \%$. In the 48 hours post-extubation, NIV was used at least 8 hours/day.

$\begin{array}{ll}\text { Outcomes } & \text { Arterial blood gases }\left(\mathrm{pH}, \mathrm{PaCO}_{2}, \mathrm{PaO}_{2} / \mathrm{FiO}_{2}\right) \text {; vital signs (heart rate, MAP, respiratory rate) at } 3 \text { hours, } 24 \\ & \text { hours, and } 48 \text { hours after extubation; duration of respiratory support; length of ICU stay; all-cause mor- } \\ & \text { tality at } 28 \text { days; respiratory failure (needing either NIV or reintubation); comfort score (scale } 0 \text { to } 10= \\ & \text { very comfortable to very uncomfortable); adverse events (aspiration, and facial breakdown); need for } \\ & \text { bronchoscopy }\end{array}$

Notes

Funding/declarations of interest: funded by National Fund of China

Study dates: January 2017 to July 2018

\section{Risk of bias}

\begin{tabular}{|c|c|c|}
\hline Bias & Authors' judgement & Support for judgement \\
\hline $\begin{array}{l}\text { Random sequence genera- } \\
\text { tion (selection bias) }\end{array}$ & Low risk & Use of a predetermined random number table \\
\hline $\begin{array}{l}\text { Allocation concealment } \\
\text { (selection bias) }\end{array}$ & Low risk & $\begin{array}{l}\text { Randomization sheet was kept by a secretary who was not otherwise involved } \\
\text { in the study. }\end{array}$ \\
\hline $\begin{array}{l}\text { Blinding of participants } \\
\text { and personnel (perfor- } \\
\text { mance bias) } \\
\text { All outcomes }\end{array}$ & Unclear risk & $\begin{array}{l}\text { Blinding was not possible. Although we expected that this would not influence } \\
\text { outcome data, we could not be certain of this. }\end{array}$ \\
\hline $\begin{array}{l}\text { Blinding of outcome as- } \\
\text { sessors (objective out- } \\
\text { comes) }\end{array}$ & Low risk & $\begin{array}{l}\text { Blinding of outcome assessors was not described; we did not anticipate that } \\
\text { this would influence the assessment of objective outcome measures. }\end{array}$ \\
\hline $\begin{array}{l}\text { Blinding of outcome as- } \\
\text { sessors (subjective mea- } \\
\text { sures) }\end{array}$ & Low risk & $\begin{array}{l}\text { Participants were outcome assessors for comfort and used a standardized } \\
\text { approach: we did not anticipate that this would influence the assessment of } \\
\text { these outcomes. }\end{array}$ \\
\hline $\begin{array}{l}\text { Incomplete outcome data } \\
\text { (attrition bias) } \\
\text { All outcomes }\end{array}$ & Low risk & Two losses in the intervention group. Study authors reported ITT analysis. \\
\hline $\begin{array}{l}\text { Selective reporting (re- } \\
\text { porting bias) }\end{array}$ & Unclear risk & $\begin{array}{l}\text { Study was retrospectively registered with a clinical trials register } \\
\text { (NCT03458364). It was not feasible to effectively assess risk of selective report- } \\
\text { ing bias with these documents. }\end{array}$ \\
\hline
\end{tabular}


Jing 2019 (Continued)

Other bias Low risk We identified no other sources of bias.

Lee 2018

\section{Study characteristics}

\begin{tabular}{|c|c|}
\hline Methods & $\mathrm{RCT}$, cross-over design. Single-centre study \\
\hline Participants & 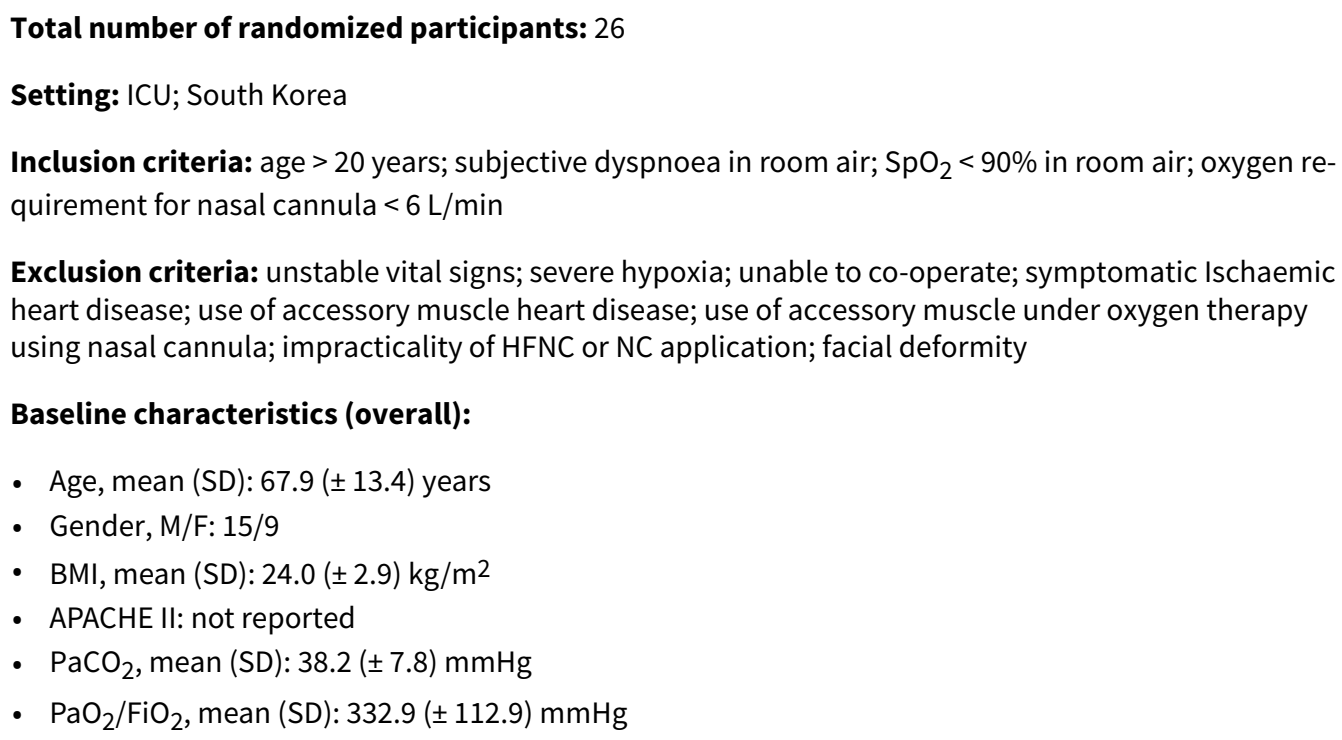 \\
\hline
\end{tabular}

Interventions Cross-over study design, participants received each of two therapies for 20 minutes each:

- HFNC: oxygen delivered using OmniOx System, initial gas flow rate at $35 \mathrm{~L} / \mathrm{min}$ with $\mathrm{FiO}_{2}$ at $35 \%$ $\mathrm{mmHg}$, then adjusted at discretion of the physician. One participant only received $15 \mathrm{~L} / \mathrm{min}$.

- Oxygen via conventional nasal cannula: no additional details

Randomized, $\mathrm{n}=26$; losses, $\mathrm{n}=2$ (withdrew consent); analysed, $\mathrm{n}=24$

Outcomes $\quad$ Flow rates; tidal volumes; comfort (10-point scale)

Note: we did not report outcome data in the review because study authors did not include outcome data from the first period.

Notes Funding/declarations of interest: Draeger provided EIT monitor. Study author declared no potential conflict of interest, and funder had no role in the design, collection, analysis or interpretation of the study.

Study dates: 1 October 2014 to 28 February 2015

Note: we did not conduct 'Risk of bias' assessments for this study because we did not report outcome data in the review.

\section{Study characteristics}


Lemiale 2015 (Continued)

Methods RCT, parallel-group design, multicentre study

Participants
Total number of participants: 102

Setting: 4 ICUs; France

Inclusion criteria: consecutive immunocompromised patients admitted to ICU for acute respiratory failure, aged $>18$ years

Exclusion criteria: hypercapnia ( $>45 \mathrm{mmHg}$ ), mechanical ventilation before ICU admission, need for immediate NIV or invasive mechanical ventilation, and patient refusal to participate in study

\section{Baseline characteristics:}

Intervention group (HFNC):

- Age, median (IQR): 59.3 (43 to 70 ) years

- Gender, M/F: 38/14

- BMI mean (SD): not reported

- SAPS II, median (IQR): 42 (29.5 to 52)

- SOFA, median (IQR): 3.5 (2 to 6)

- $\mathrm{PaCO}_{2}$ : not reported

- $\mathrm{PaO}_{2} / \mathrm{FiO}_{2}$, median (IQR): 128 (48 to 178 ) $\mathrm{mmHg}$

- Respiratory rate, median (IQR): 26 (21.7 to 31.2 ) breaths/min

Control group (standard oxygen therapy):

- Age, median (IQR): 64.5 (53.25 to 72 ) years

- Gender, M/F: 32/16

- BMI mean (SD): not reported

- SAPS II, median (IQR): 37.5 (31.5 to 46.5 )

- SOFA, median (IQR): 3 (2 to 5 )

- $\mathrm{PaCO}_{2}$ : not reported

- $\mathrm{PaO}_{2} / \mathrm{FiO}_{2}$ median (IQR): 100 (40 to 156$) \mathrm{mmHg}$

- Respiratory rate median (IQR): 27 (22 to 32.2 ) breaths/min

Interventions Intervention group:

- Randomized, $n=53$; losses, $n=1$ (1 withdrew consent); analysed, $n=52$

- Details: HFNC; heated, humidified circuit, with initial flow of 40 to $50 \mathrm{~L} / \mathrm{min}$; $\mathrm{FiO}_{2} 100 \%$, which was then adjusted to maintain $\mathrm{SpO}_{2} \geq 95 \%$

Control group

- Randomized, $\mathrm{n}=49$; losses, $\mathrm{n}=1$ (1 withdrew consent); analysed, $\mathrm{n}=48$

- Details: Venturi mask; $\mathrm{FiO}_{2}$ initially $60 \%, 15 \mathrm{~L} / \mathrm{min}$, then adjusted to maintain $\mathrm{SpO}_{2} \geq 95 \%$

Participants were randomly allocated to oxygen therapy groups for a 2-hour period.

Outcomes

Need for invasive mechanical ventilation or NIV during or at the end of the 2-hour study period; VAS scores for comfort, thirst, and dyspnoea (all at 120 minutes); respiratory rate (at 120 minutes); heart rate

Notes

Funding/declarations of interest: Fisher \& Paykel Healthcare Ltd provided oxygen delivery devices and funds for study insurance and presentation of results. The sponsors had no role in designing or conducting the study.

Study dates: November 2012 to April 2014 
Note: we noted some differences in baseline characteristics but these were not clinically significant.

\section{Risk of bias}

\begin{tabular}{|c|c|c|}
\hline Bias & Authors' judgement & Support for judgement \\
\hline $\begin{array}{l}\text { Random sequence genera- } \\
\text { tion (selection bias) }\end{array}$ & Low risk & $\begin{array}{l}\text { Participants described as randomly allocated, with stratification on study cen- } \\
\text { tre by permuted block method }\end{array}$ \\
\hline $\begin{array}{l}\text { Allocation concealment } \\
\text { (selection bias) }\end{array}$ & Low risk & Use of opaque, sealed envelopes to ensure identity concealment \\
\hline $\begin{array}{l}\text { Blinding of participants } \\
\text { and personnel (perfor- } \\
\text { mance bias) } \\
\text { All outcomes }\end{array}$ & Low risk & $\begin{array}{l}\text { Blinding was not possible. Although we expected that this would not influence } \\
\text { outcome data, we could not be certain of this. }\end{array}$ \\
\hline $\begin{array}{l}\text { Blinding of outcome as- } \\
\text { sessors (objective out- } \\
\text { comes) }\end{array}$ & Low risk & $\begin{array}{l}\text { Blinding of outcome assessors was not described; we did not anticipate that } \\
\text { this would influence the assessment of objective outcome measures. }\end{array}$ \\
\hline $\begin{array}{l}\text { Blinding of outcome as- } \\
\text { sessors (subjective mea- } \\
\text { sures) }\end{array}$ & Low risk & $\begin{array}{l}\text { Standardized approach to measuring subjective outcomes of comfort, thirst } \\
\text { and dyspnoea: we did not anticipate that this would influence the assessment } \\
\text { of these outcomes. }\end{array}$ \\
\hline $\begin{array}{l}\text { Incomplete outcome data } \\
\text { (attrition bias) } \\
\text { All outcomes }\end{array}$ & Low risk & $\begin{array}{l}\text { Loss of two participants after randomization due to withdrawal of consent. } \\
\text { Low number, unlikely to influence results }\end{array}$ \\
\hline $\begin{array}{l}\text { Selective reporting (re- } \\
\text { porting bias) }\end{array}$ & Unclear risk & $\begin{array}{l}\text { NCT02424773. Retrospective registration in April } 2015 \text {. Therefore, not feasible } \\
\text { to judge if any reporting bias. All outcomes reported from methods section }\end{array}$ \\
\hline Other bias & Low risk & We identified no other risks of bias. \\
\hline
\end{tabular}

Longhini 2019

\section{Study characteristics}

Methods RCT, cross-over design. Multicentre study

\section{Participants}

\section{Total number of randomized participants: 32}

Setting: 2 ICUs; Italy

Inclusion criteria: COPD, NIV > 24 hours; fully co-operative; $\mathrm{pH} \geq 7.35$ during NIV; respiratory rate $\leq 30$ breaths/min; improvement of condition (no dyspnoea, no agitation, no fever)

Exclusion criteria: diaphragm paralysis; clinical signs of distress or impending respiratory muscle failure; haemodynamic instability; life-threatening cardiac arrhythmia; ECG signs of ischaemia; impaired renal function; inclusion in other studies; refusal to consent

\section{Baseline characteristics (overall, for analysed participants only):}

- Age, mean (SD): $72.5( \pm 8.2)$ years

- Gender, M/F: 17/13

- BMI, mean (SD): not reported

- SAPS II, mean (SD) : $31.5( \pm 6.2)$ 
Longhini 2019 (Continued)

- $\mathrm{PaCO}_{2}$, mean (SD): not reported

- $\mathrm{PaO}_{2} / \mathrm{FiO}_{2}$, mean (SD): not reported

Interventions

Cross-over study design, with 30 minutes for each 5 intervention stages. Three intervention stages, which were all NIV, were not randomized. Two randomized interventions were given as interruptions to the NIV stages:

- HFNO delivered using Optiflow via nasal cannula connected to a heated humidifier (MR850)

- Standard oxygen treatment using Venturi mask

Randomized participants, $n=32$; losses, $n=2$ (insufficient ultrasound imaging quality); analysed, $n=30$

Outcomes Evaluation of right hemidiaphragm; respiratory rate; $\mathrm{pH} ; \mathrm{PaO}_{2} ; \mathrm{PaCO}_{2} ;$ comfort (using 11-point NRS)

Note: we did not include outcome data in the review because study authors did not report outcome data from the first period.

Notes

Funding/declarations of interest: some authors received fees or institutional funding from one or more of the following: Chiesi; AIM ITALY SRL; Fisher and Paykel Healthcare Ltd; Maquet Critical Care; Draeger; Intersurgical SpA; Orionpharma; Phils; Resmed; Merck Sharp and Dohme; Novartis

Study dates: December 2015 to March 2017

Note: we did not complete 'Risk of bias' assessments for this study because we did not report outcome data in the review.

\section{Study characteristics}

\begin{tabular}{|c|c|}
\hline Methods & $\mathrm{RCT}$, parallel-group design. Multicentre study \\
\hline Participants & 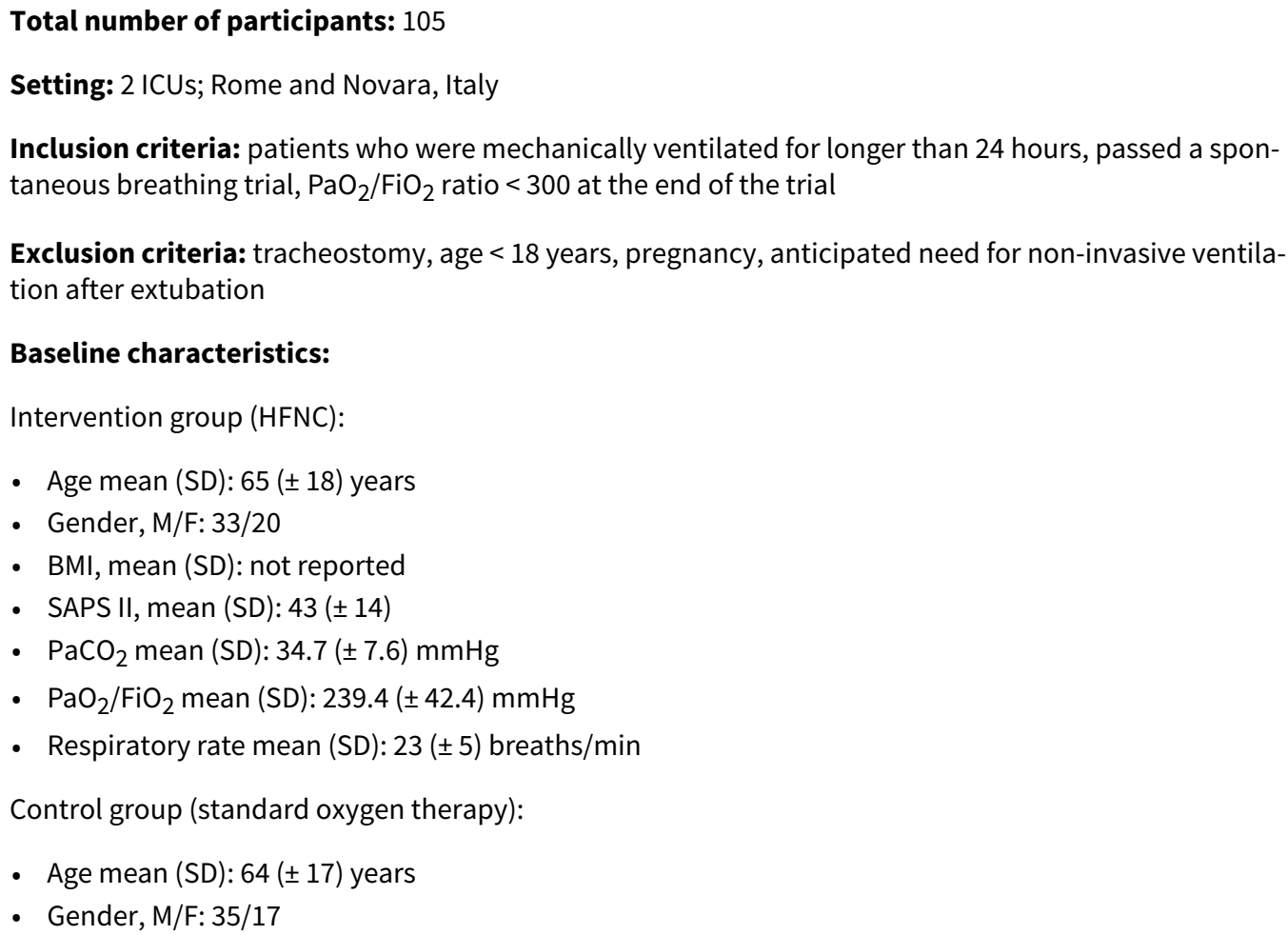 \\
\hline
\end{tabular}


Maggiore 2014 (Continued)

- BMI, mean (SD): not reported

- SAPS II, mean (SD): $44( \pm 16)$

- $\mathrm{PaCO}_{2}$ mean (SD): $36( \pm 7.1) \mathrm{mmHg}$

- $\mathrm{PaO}_{2} / \mathrm{FiO}_{2}$ mean (SD): 241.7 ( \pm 51.1$) \mathrm{mmHg}$

- Respiratory rate mean (SD): 23 (6) breaths/min

\begin{tabular}{ll}
\hline Interventions & Intervention group (HFNC): \\
- Randomized, $\mathrm{n}=53$; losses, $\mathrm{n}=0$; analysed, $\mathrm{n}=53$ \\
- Details: flow $=50 \mathrm{~L} / \mathrm{min}$. Optiflow, Fisher \& Paykel Healthcare, Auckland, New Zealand \\
Control group (Venturi mask): \\
- Randomized, $\mathrm{n}=52$; losses, $\mathrm{n}=0$; analysed, $\mathrm{n}=52$ \\
- Details: Venturi mask to deliver predetermined $\mathrm{FiO}_{2}$ \\
Both used after extubation. FiO ${ }_{2}$ was set to obtain $\mathrm{SpO}_{2} 92 \%$ to $98 \%$ (88\% to 95\% in COPD). Applied for \\
48 hours or until ICU discharge
\end{tabular}

\begin{tabular}{ll}
\hline Outcomes & Arterial blood gas, $\mathrm{SaO}_{2}, \mathrm{FiO}_{2}, \mathrm{PaO}_{2} / \mathrm{FiO}_{2}$ ratio, respiratory rate, MAP, heart rate, and discomfort \\
(recorded at $1,3,6,12,24,36$, and 48 hours), $\mathrm{PaCO}_{2}$ at 3 hours. Adverse events (displacement of oxy- \\
genation device, oxygen desaturation post-extubation requiring NIV or endotracheal intubation). ICU \\
length of stay and mortality \\
Note: we did not include data for $\mathrm{SaO}_{2}$ because these data were presented in figures which we could \\
not clearly translate into numerical data.
\end{tabular}

Notes

Funding/declarations of interest: supported by an unrestricted research grant from Fisher \& Paykel Healthcare Ltd and by an independent research grant

Study dates: November 2010 to April 2011

Note: there are 3 secondary references to this study (conference reports).

\section{Risk of bias}

\begin{tabular}{|c|c|c|}
\hline Bias & Authors' judgement & Support for judgement \\
\hline $\begin{array}{l}\text { Random sequence genera- } \\
\text { tion (selection bias) }\end{array}$ & Low risk & A unique random number sequence that was computer generated \\
\hline $\begin{array}{l}\text { Allocation concealment } \\
\text { (selection bias) }\end{array}$ & Unclear risk & $\begin{array}{l}\text { Participants were randomly assigned, using a block size of } 30 \text {, to Optiflow or } \\
\text { Venturi mask in a blinded fashion with opaque envelopes - no specific mention } \\
\text { as to whether the envelopes were consecutively numbered. }\end{array}$ \\
\hline $\begin{array}{l}\text { Blinding of participants } \\
\text { and personnel (perfor- } \\
\text { mance bias) } \\
\text { All outcomes }\end{array}$ & Unclear risk & $\begin{array}{l}\text { Blinding was not possible. Although we expected that this would not influence } \\
\text { outcome data, we could not be certain of this. }\end{array}$ \\
\hline $\begin{array}{l}\text { Blinding of outcome as- } \\
\text { sessors (objective out- } \\
\text { comes) }\end{array}$ & Low risk & $\begin{array}{l}\text { Database monitored by independent third parties, analysis performed as } \\
\text { agreed before commencement of the study. However, we assumed that out- } \\
\text { come assessors were not blinded; we did not anticipate that this would influ- } \\
\text { ence the assessment of objective outcome measures. }\end{array}$ \\
\hline $\begin{array}{l}\text { Blinding of outcome as- } \\
\text { sessors (subjective mea- } \\
\text { sures) }\end{array}$ & Low risk & $\begin{array}{l}\text { Participants were the outcome assessors for discomfort using a standardized } \\
\text { visual scale: we did not expect this to influence the outcome data. }\end{array}$ \\
\hline
\end{tabular}


Maggiore 2014 (Continued)

Incomplete outcome data Low risk No apparent losses
(attrition bias)

All outcomes

Selective reporting (re- Low risk porting bias)

NCT01575353. Retrospectively registered in December 2010 (but only shortly before start of recruitment). All outcomes reported as stated in protocol. Length of stay and mortality rates reported but not previously stated in protocol

\begin{tabular}{lll}
\hline Other bias $\quad$ Unclear risk $\quad$ Multiple interim analyses performed (3 abstracts presented from same study) \\
\hline
\end{tabular}

Mauri 2017a

\section{Study characteristics}

\begin{tabular}{|c|c|}
\hline Methods & $\mathrm{RCT}$, cross-over design. Single-centre study \\
\hline \multirow[t]{12}{*}{ Participants } & Total number of randomized participants: 17 \\
\hline & Setting: ICU; Italy \\
\hline & $\begin{array}{l}\text { Inclusion criteria: non-intubated people with AHRF admitted to the ICU; new or worsening respiratory } \\
\text { symptoms following a known clinical insult lasting }<1 \text { week; } \mathrm{PaO}_{2} / \mathrm{FiO}_{2} \leq 300 \mathrm{mmHg} \text { while receiving ad- } \\
\text { ditional oxygen; evidence of pulmonary infiltrates }\end{array}$ \\
\hline & $\begin{array}{l}\text { Exclusion criteria: < } 18 \text { years of age; presence of tracheostomy; pregnancy or breastfeeding; haemo- } \\
\text { dynamic instability; evidence of pneumothorax; respiratory failure explained by cardiac failure or flu- } \\
\text { id overload; severe COPD; history of nasal trauma and/or deviated nasal septum; altered mental sta- } \\
\text { tus; contraindications to EIT; impossibility to position the EIT belt or position the oesophageal pressure } \\
\text { catheter }\end{array}$ \\
\hline & Baseline characteristics (overall): \\
\hline & - Age, mean (SD): $62( \pm 10)$ years \\
\hline & - Gender, M/F: 8/9 \\
\hline & - BMI, mean (SD): not reported \\
\hline & - SAPS II, mean (SD): $48( \pm 13)$ \\
\hline & - SOFA score, mean (SD): $11( \pm 3)$ \\
\hline & - $\mathrm{PaCO}_{2}$, mean (SD): not reported \\
\hline & - $\mathrm{PaO}_{2} / \mathrm{FiO}_{2}$, mean (SD): $167( \pm 46) \mathrm{mmHg}$ \\
\hline \multirow[t]{7}{*}{ Interventions } & $\begin{array}{l}\text { Cross-over study design, with each phase lasting } 20 \text { minutes. Washout period was not specified (but not } \\
\text { relevant to this review as we were considering only data from the first study period). }\end{array}$ \\
\hline & - HFNC: gas flow at $30 \mathrm{~L} / \mathrm{min}$ \\
\hline & - HFNC: gas flow at $45 \mathrm{~L} / \mathrm{min}$ \\
\hline & - HFNC: gas flow at $60 \mathrm{~L} / \mathrm{min}$ \\
\hline & - Standard non-occlusive oxygen facemask: gas flow at $12 \mathrm{~L} / \mathrm{min}$ \\
\hline & HFNC was delivered through specific nasal prongs to fit the size of the nostrils. $\mathrm{FiO}_{2}$ to achieve target \\
\hline & $\mathrm{SpO}_{2}$ of 90 to $95 \%$ \\
\hline
\end{tabular}

\section{Outcomes}

Blood gas analysis $\left(\mathrm{PaO}_{2} ; \mathrm{PaO}_{2} / \mathrm{FiO}_{2} ; \mathrm{PaCO}_{2}\right)$; respiratory rate; haemodynamics 
Notes Funding/declarations of interest: funding not reported. Study authors declared no competing interests.

Study dates: not reported

\section{Study characteristics}

\begin{tabular}{|c|c|}
\hline Methods & $\mathrm{RCT}$, cross-over design. Single-centre study \\
\hline Participants & 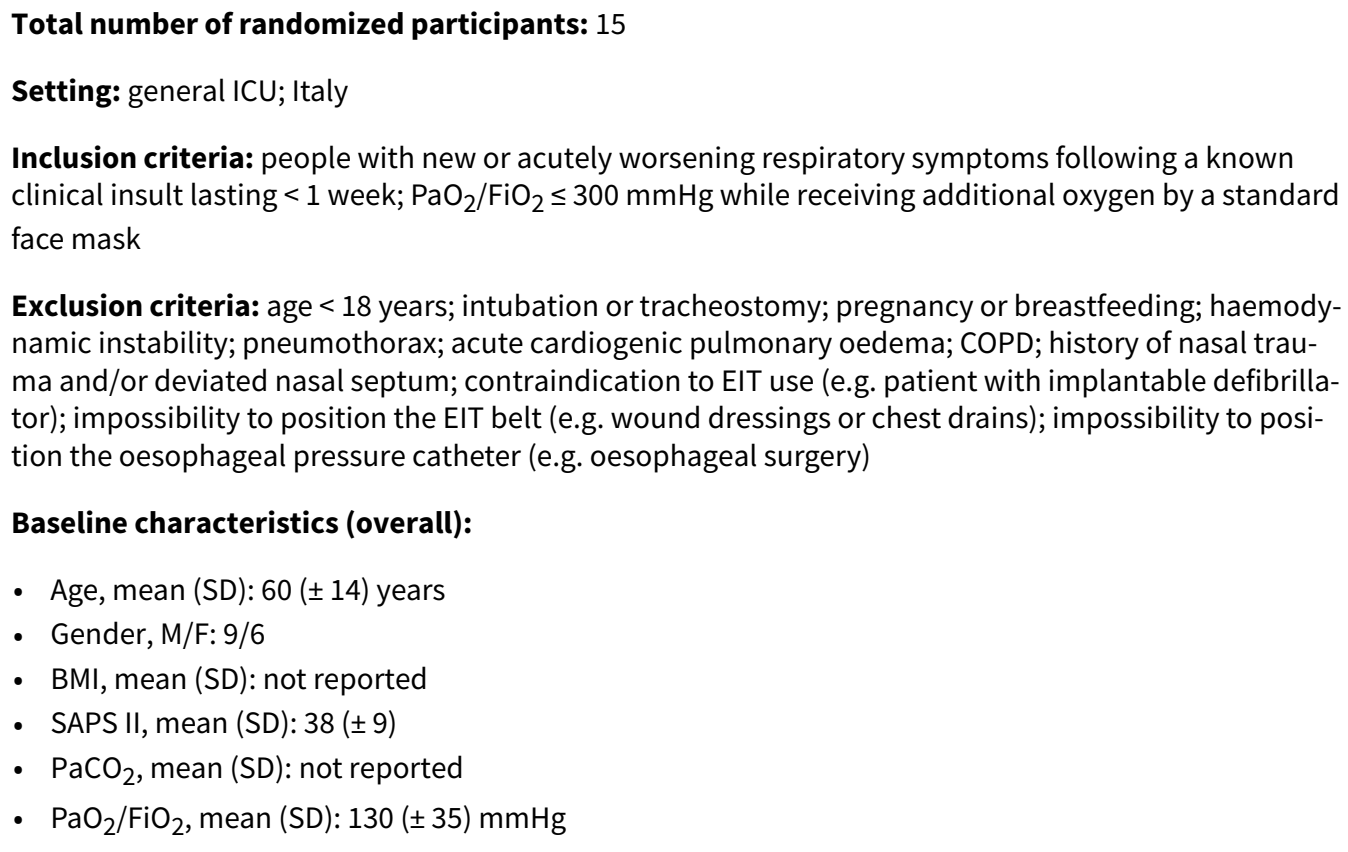 \\
\hline
\end{tabular}

Interventions

Cross-over study with each study phase lasting for 20 minutes.

- HFNC: via AIRVO 2, gas flow at $40 \mathrm{~L} / \mathrm{min}$

- standard non-occlusive oxygen facial mask, with gas flow set at $12 \mathrm{~L} / \mathrm{min}$

$\mathrm{FiO}_{2}$ to achieve $\mathrm{SpO}_{2}$ between $90 \%$ and $95 \%$

Outcomes Arterial blood gases; respiratory rate; haemodynamics; EIT parameters

Note: we did not include outcome data in the review because the study authors did not report outcome data from the first study period.

Notes

Funding/declarations of interest: institutional funding of the Department of Medicine, University of Milan-Bicocca, Monza, Italy. Fisher and Paykel Healthcare, Auckland, New Zealand, provided the device and disposables to deliver high-flow nasal cannula therapy free of charge but had no role in the conception, design and conduct of the study, data analysis, and writing of the manuscript. The authors reported no conflicts of interest.

Study dates: not specified 
Parke 2011

\section{Study characteristics}

\begin{tabular}{ll}
\hline Methods & RCT, parallel-group design. Single-centre study \\
\hline Participants & Total number of randomized participants: 56 \\
& Setting: cardiothoracic and vascular ICU; Auckland, New Zealand \\
& Inclusion criteria: patients in a cardiothoracic and vascular ICU with mild to moderate hypoxaemic \\
& respiratory failure defined by study authors as follows: receiving $\geq 4 \mathrm{~L} / \mathrm{min}$ of oxygen via nasal cannu- \\
& la for longer than 4 hours and/or respiratory rate $\geq 25$ breaths $/$ min and/or increased work of breathing, \\
& evidenced by clinical signs such as dyspnoea, in-drawing, accessory muscle use, and diaphoresis; or re- \\
ceiving $\geq 6$ L/min of oxygen via face mask for longer than 2 hours, or respiratory rate $\geq 25$ breaths/min, \\
or both, or increased work of breathing, as evidenced by clinical signs such as dyspnoea, in-drawing, \\
accessory-muscle use, and diaphoresis, or both
\end{tabular}

Exclusion criteria: patients requiring imminent mechanical ventilation and those under orders to not receive mechanical ventilation

\section{Baseline characteristics:}

Intervention group (HFNC):

- Age, mean (range): 64 (39 to 83) years

- Gender, M/F: 23/4

- BMI, mean (SD): not reported

- APACHE II, mean (range): 12 (5 to 25)

- $\mathrm{PaCO}_{2}$, mean (SD): $43( \pm 7) \mathrm{mmHg}$

- $\mathrm{PaO}_{2} / \mathrm{FiO}_{2}$ : not reported

- Respiratory rate, mean (SD): $21( \pm 7)$ breaths/min

Control group (standard oxygen therapy):

- Age, mean (range): 64 (26 to 85 ) years

- Gender, M/F:21/8

- BMI, mean (SD): not reported

- APACHE II, mean (range): 12 ( 1 to 21)

- $\mathrm{PaCO}_{2}$, mean (SD): $42( \pm 7) \mathrm{mmHg}$

- $\mathrm{PaO}_{2} / \mathrm{FiO}_{2}$ : not reported

- Respiratory rate, mean (SD): 18 ( \pm 8 ) breaths/min

- Randomized, $\mathrm{n}=30$; losses, $\mathrm{n}=1$ (refused consent); analysed, $\mathrm{n}=29$

- Details: HFNC; Optiflow, Fisher \& Paykel Healthcare, with MR880 humidifier, RT241 heated delivery tube, and RT033 large/RT034 small, wide-bore nasal cannula; therapy commenced at an initial flow of $35 \mathrm{~L} / \mathrm{min}$; flow and $\mathrm{FiO}_{2}$ titrated to $\mathrm{SpO}_{2}$ or $\mathrm{SaO}_{2}$ of $95 \%$. Duration of oxygen therapy not reported

Control group (standard oxygen therapy):

- Randomized, $\mathrm{n}=30$; losses, $\mathrm{n}=3$ ( 1 refused consent, 2 failed screening); analysed, $\mathrm{n}=27$

- Details: HFFM (standard face mask, MR850 humidifier, RT308 heated delivery tube and air entrainer, Fisher \& Paykel Healthcare) with an aerosol mask (HudsonRCI, TFX Medical, High Wycombe, UK); flow rate $\leq 15 \mathrm{~L} / \mathrm{min}$; humidified oxygen delivered at $31^{\circ} \mathrm{C}$ and $32 \mathrm{mg} \mathrm{H} \mathrm{H}_{2} \mathrm{O} / \mathrm{L}$; titrated to an $\mathrm{SpO}_{2}$ or $\mathrm{SaO}_{2}$ $95 \%$. Duration of oxygen therapy not reported 
Parke 2011 (Continued)

Outcomes

Assessment score, arterial blood gas values, $\mathrm{SpO}_{2}$, respiratory rate, and heart rate at baseline, $30 \mathrm{~min}$ utes, 1 hour, 2 hours, and 4 hours after randomization, then as per unit protocol. Continuous $\mathrm{SpO}_{2}$ data and instances of desaturation ( $\mathrm{SpO}_{2} 93 \%$ for longer than 5 seconds) were collected. Episodes were discounted if the $\mathrm{SpO}_{2}$ trace indicated signal interference or signal loss. Allocated therapy was considered successful if participants were maintained on or were weaned from their assigned oxygen therapy within 24 hours of enrolment. Failure of therapy was defined as worsening respiratory failure that required a change in the respiratory support device within 24 hours of study enrolment.

Notes

Funding/declarations of interest: Fisher \& Paykel Healthcare Ltd consulted regarding study design and data analysis, and paid for statistical analysis.

Study dates: not reported

Note: some additional outcome data retrieved through email contact with study authors

\section{Risk of bias}

\begin{tabular}{|c|c|c|}
\hline Bias & Authors' judgement & Support for judgement \\
\hline $\begin{array}{l}\text { Random sequence genera- } \\
\text { tion (selection bias) }\end{array}$ & Low risk & Random numbers table \\
\hline $\begin{array}{l}\text { Allocation concealment } \\
\text { (selection bias) }\end{array}$ & Unclear risk & Opaque sealed envelopes but no mention of whether numbered or not \\
\hline $\begin{array}{l}\text { Blinding of participants } \\
\text { and personnel (perfor- } \\
\text { mance bias) } \\
\text { All outcomes }\end{array}$ & Unclear risk & $\begin{array}{l}\text { Blinding was not possible. Although we expected that this would not influence } \\
\text { outcome data, we could not be certain of this. }\end{array}$ \\
\hline $\begin{array}{l}\text { Blinding of outcome as- } \\
\text { sessors (objective out- } \\
\text { comes) }\end{array}$ & Low risk & $\begin{array}{l}\text { Blinding of outcome assessors was not described; we did not anticipate that } \\
\text { this would influence the assessment of objective outcome measures. }\end{array}$ \\
\hline $\begin{array}{l}\text { Incomplete outcome data } \\
\text { (attrition bias) } \\
\text { All outcomes }\end{array}$ & Unclear risk & $\begin{array}{l}\text { Of } 60 \text { enrolled in the study, } 4 \text { participants ( } 1 \text { from the HFNC group, and } 3 \text { from } \\
\text { the HFFM group) were excluded: } 2 \text { refused consent for all data collection and } \\
2 \text { failed the screening. Five of } 27 \text { participants in the high-flow face mask group } \\
\text { were switched to nasal high flow - no mention of how these data were treated }\end{array}$ \\
\hline $\begin{array}{l}\text { Selective reporting (re- } \\
\text { porting bias) }\end{array}$ & High risk & $\begin{array}{l}\text { ACTRN012606000139572. Prospective registration in April 2006. Published } \\
\text { study reported additional outcomes (to include respiratory failure) that were } \\
\text { not stated in trial registration records; this may indicate selective reporting } \\
\text { bias for these outcomes. }\end{array}$ \\
\hline Other bias & Low risk & We noted no other sources of bias. \\
\hline
\end{tabular}

\section{Parke 2013a}

\section{Study characteristics}

\begin{tabular}{ll}
\hline Methods & RCT, parallel-group design. Single-centre \\
\hline
\end{tabular}

Participants

\section{Total number of randomized participants: 340}

Setting: ICU; Auckland, New Zealand 
Parke 2013a (Continued)

Inclusion criteria: undergoing elective cardiac surgery utilizing cardiopulmonary bypass were eligible for inclusion in this study if $\geq 18$ years of age and undergoing surgery involving full median sternotomy

Exclusion criteria: contraindication to HFNC, e.g. presence of a nasal septal defect, and previous recruitment

\section{Baseline characteristics:}

Intervention group (HFNC):

- Age, median (range): 65 (19 to 88 ) years

- Gender, M/F: $129 / 40$

- BMI, mean (SD): $28.4( \pm 5.3) \mathrm{kg} / \mathrm{m}^{2}$

- APACHE II, mean (SD): not reported

- $\mathrm{PaCO}_{2}$ : not reported

- $\mathrm{PaO}_{2} / \mathrm{FiO}_{2}$ : not reported

- Respiratory rate, mean (SD): 16.6 ( \pm 1.9$)$ breaths/min

Control group (simple face mask):

- Age, median (range): 66 (21 to 87) years

- Gender, M/F: 129/42

- BMI, mean (SD): $29.2( \pm 5.5) \mathrm{kg} / \mathrm{m}^{2}$

- APACHE II, mean (SD): not reported

- $\mathrm{PaCO}_{2}$ : not reported

- $\mathrm{PaO}_{2} / \mathrm{FiO}_{2}$ : not reported

- Respiratory rate, mean (SD): 16.5 ( \pm 1.7$)$ breaths/min

Interventions Intervention group:

- Randomized, $\mathrm{n}=170$; losses, $\mathrm{n}=1$ (consent withdrawn); analysed, $\mathrm{n}=169$

- Details: HFNC; Optiflow system; flow rate $45 \mathrm{~L} / \mathrm{min}$

Control group:

- Randomized, $\mathrm{n}=171$; losses, $\mathrm{n}=0$; analysed, $\mathrm{n}=171$

- Details: Simple face mask; oxygen at 2 to $4 \mathrm{~L} / \mathrm{min}$ via simple face mask or nasal prongs; $\mathrm{FiO}_{2}$ in both groups was titrated to maintain $\mathrm{SpO}_{2}>93 \%$.

Oxygen therapy started after extubation.

Outcomes

Number of participants with $\mathrm{SpO}_{2} / \mathrm{FiO}_{2}$ ratio $\geq 445$ on day 3 after cardiac surgery; atelectasis score of chest X-rays; spirometry; re-admission to ICU for respiratory causes; ICU and hospital length of stay; duration of respiratory support; mortality; incidence of respiratory complications on day 28; respiratory rate; oxygenation; use of adjunctive respiratory support therapies; escalation of respiratory support; adverse events; patient comfort

Notes

Funding/declarations of interest: Study authors declared that research was supported by an unrestricted grant from Fisher \& Paykel Healthcare Ltd, but that the sponsors had no part in the study design and no access to trial data.

Study dates: not reported. Conducted over a 14-month period

Note: some additional outcome data retrieved through email contact with study authors

\section{Risk of bias}


Parke 2013a (Continued)

Random sequence genera- Low risk Computer-generated random numbers in blocks of 12 tion (selection bias)

Allocation concealment $\quad$ Low risk
(selection bias)

(selection bias)

\author{
Blinding of participants Unclear risk \\ and personnel (perfor- \\ mance bias) \\ All outcomes
}

Blinding was not possible. Although we expected that this would not influence outcome data, we could not be certain of this.

\begin{tabular}{|c|c|c|}
\hline $\begin{array}{l}\text { Blinding of outcome as- } \\
\text { sessors (objective out- }\end{array}$ & Low risk & $\begin{array}{l}\text { Blinding of outcome assessors was not described; we did not anticipate that } \\
\text { this would influence the assessment of objective outcome measures. }\end{array}$ \\
\hline
\end{tabular}
comes)

\begin{tabular}{|c|c|c|}
\hline $\begin{array}{l}\text { Blinding of outcome as- } \\
\text { sessors (subjective mea- }\end{array}$ & Low risk & $\begin{array}{l}\text { Participants were outcome assessors for comfort scores: we did not expect } \\
\text { blinding to influence the assessment of these outcome data. }\end{array}$ \\
\hline
\end{tabular}
sures)

\begin{tabular}{lll}
\hline $\begin{array}{l}\text { Incomplete outcome data } \\
\text { (attrition bias) } \\
\text { All outcomes }\end{array}$ & Low risk & Attrition fully reported. Small number of losses \\
\hline $\begin{array}{l}\text { Selective reporting (re- } \\
\text { porting bias) }\end{array}$ & High risk & $\begin{array}{l}\text { ACTRN12610000973011. Prospective registration in November 2010. Published } \\
\text { study reported additional outcomes that were not stated in trial registration } \\
\text { records (ICU and hospital length of stay, duration of respiratory support, oxy- } \\
\text { genation, escalation of respiratory support, adverse events); this may indicate } \\
\text { selective reporting bias for these outcomes. }\end{array}$ \\
\hline
\end{tabular}

Other bias Low risk We identified no other sources of bias.

Rittayamai 2014

\section{Study characteristics}

\begin{tabular}{|c|c|}
\hline Methods & RCT, cross-over design. Single-centre \\
\hline Participants & $\begin{array}{l}\text { Total number of randomized participants: } 17 \\
\text { Setting: respiratory ICU; Bangkok, Thailand } \\
\text { Inclusion criteria: mechanically ventilated patients who were } 18 \text { years of age, successfully weaned by } \\
\text { spontaneous breathing, trial with oxygen T-piece or low level of pressure support for } 120 \text { minutes, and } \\
\text { ready for endotracheal extubation } \\
\text { Exclusion criteria: haemodynamic instability or decreased level of consciousness; patients who lacked } \\
\text { co-operation, tracheotomized patients, and pregnant women } \\
\text { Baseline characteristics: } \\
\text { - Age, mean (SD): } 66.8( \pm 13.8) \text { years } \\
\text { - Gender, M/F: } 10 / 7 \\
\text { - BMI, mean }(S D): \text { not reported } \\
\text { - SAPS II, mean (SD): } 30.9( \pm 4.4) \\
\text { - Respiratory rate, mean }(S D): \text { recorded before each cross-over period: baseline } 1: 20.3( \pm 4.5) \text {; baseline } \\
2: 21.7( \pm 3.8) \text { breaths/min }\end{array}$ \\
\hline
\end{tabular}


Rittayamai 2014 (Continued)

- $\mathrm{PaCO}_{2}:$ not reported

- $\mathrm{PaO}_{2} / \mathrm{FiO}_{2}$ : not reported

Interventions Intervention group (HFNC):

- Details: HFNC, Optiflow system, Fisher \& Paykel Healthcare; initial inspiratory flow of $35 \mathrm{~L} / \mathrm{min}$, and $\mathrm{FiO}_{2}$ adjusted to achieve $\mathrm{SpO}_{2} \geq 94 \%$ within the first 5 minutes and to maintain this setting for 30 minutes

Control group (standard oxygen therapy):

- Details: Non-rebreather face mask, 6 to $10 \mathrm{~L} / \mathrm{min}$ to achieve $\mathrm{SpO}_{2} 94 \%$ for another 30 minutes

Outcomes Dyspnoea, patient comfort, breathing frequency, heart rate blood pressure, $\mathrm{SpO}_{2}$

Notes

Funding/declarations of interest: study authors did not report funding sources. They disclosed no conflicts of interest.

Study dates: August to December 2011

\section{Risk of bias}

\begin{tabular}{|c|c|c|}
\hline Bias & Authors' judgement & Support for judgement \\
\hline $\begin{array}{l}\text { Random sequence genera- } \\
\text { tion (selection bias) }\end{array}$ & Unclear risk & Methods used to generate group allocation not stated \\
\hline $\begin{array}{l}\text { Allocation concealment } \\
\text { (selection bias) }\end{array}$ & Unclear risk & Methods of allocation concealment not stated \\
\hline $\begin{array}{l}\text { Blinding of participants } \\
\text { and personnel (perfor- } \\
\text { mance bias) } \\
\text { All outcomes }\end{array}$ & Unclear risk & $\begin{array}{l}\text { Blinding was not possible. Although we expected that this would not influence } \\
\text { outcome data, we could not be certain of this. }\end{array}$ \\
\hline $\begin{array}{l}\text { Blinding of outcome as- } \\
\text { sessors (objective out- } \\
\text { comes) }\end{array}$ & Low risk & $\begin{array}{l}\text { Unable to blind outcome assessors owing to nature of the intervention: we did } \\
\text { not anticipate that this would influence the assessment of objective outcome } \\
\text { measures. }\end{array}$ \\
\hline $\begin{array}{l}\text { Blinding of outcome as- } \\
\text { sessors (subjective mea- } \\
\text { sures) }\end{array}$ & Low risk & $\begin{array}{l}\text { Unable to blind outcome assessors owing to nature of the intervention. Partic- } \\
\text { ipants were outcome assessors for comfort: We did not expect that this would } \\
\text { influence the outcome data. }\end{array}$ \\
\hline $\begin{array}{l}\text { Incomplete outcome data } \\
\text { (attrition bias) } \\
\text { All outcomes }\end{array}$ & Unclear risk & $\begin{array}{l}\text { No statement of how many reported. No participant numbers in tables or } \\
\text { graphs }\end{array}$ \\
\hline \multirow[t]{2}{*}{$\begin{array}{l}\text { Selective reporting (re- } \\
\text { porting bias) }\end{array}$} & Unclear risk & $\begin{array}{l}\text { Trial registration not reported in paper. Unable to establish whether outcomes } \\
\text { were reported according to pre-published protocol or trial registration docu- } \\
\text { ments }\end{array}$ \\
\hline & & $\begin{array}{l}\mathrm{SpO}_{2} \text { and mean arterial pressure not reported for all time points set out in } \\
\text { methods }\end{array}$ \\
\hline Other bias & Low risk & We identified no other risks of bias. \\
\hline
\end{tabular}


Schwabbauer 2014

\section{Study characteristics}

\begin{tabular}{ll}
\hline Methods & RCT, cross-over design. Single-centre study \\
\hline
\end{tabular}

Participants

\section{Total number of randomized participants: 14}

Setting: medical ICU; Germany

Inclusion criteria: patients with hypoxic respiratory failure $\left(\mathrm{PaO}_{2}<55 \mathrm{mmHg}\right.$ under room air)

Exclusion criteria: ventilatory failure, haemodynamic instability, cardiogenic pulmonary oedema, non-invasive ventilation contraindications, inability to co-operate

\section{Baseline characteristics (recorded before each cross-over period)}

- Age, mean (SD): $55.9( \pm 20.36)$ years

- Gender, M/F: not reported

- BMI, mean (SD): $26.71( \pm 3.99) \mathrm{kg} / \mathrm{m}^{2}$

- SAPS II, mean (SD): 41.21 ( \pm 11.78$)$

- $\mathrm{PaCO}_{2}$, mean (SD): baseline 1: $36( \pm 5)$; baseline 2: $38( \pm 5)$; baseline 3: $37( \pm 5)$

- $\mathrm{PaO}_{2} / \mathrm{FiO}_{2}$ : not reported

- Respiratory rate, mean (SD): baseline 1: $28( \pm 9)$; baseline 2: $28( \pm 9)$; baseline 3: $26( \pm 7)$ (breaths/min)

Intervention group (HFNC):

- Details: HFNC, Optiflow system, Fisher \& Paykel Healthcare; oxygen flow 55 L/min; $\mathrm{FiO}_{2}$ 0.6, using active respiratory gas humidifier

Control group one (standard oxygen therapy):

- Details: Venturi mask, Oxygen flow $15 \mathrm{~L} / \mathrm{min} ; \mathrm{FiO}_{2} 0.6$

Control group two (NIV):

- Details: Non-invasive ventilation, Intensive care ventilators in pressure support mode; PEEP set to 5 $\mathrm{cm} \mathrm{H}_{2} \mathrm{O}$; pressure support above PEEP adjusted individually to achieve tidal volume of 6 to $8 \mathrm{~mL} / \mathrm{kg}$ ideal body weight; $\mathrm{FiO}_{2} 0.6$

Each treatment phase was preceded by a 15-minute baseline phase during which participants received oxygen via a standard nasal prong (oxygen flow 4 to $12 \mathrm{~L} / \mathrm{min}, \mathrm{SaO}_{2}$ goal $\geq 88 \%$ ).

Outcomes $\quad \mathrm{PaO}_{2}$, respiratory rate, dyspnoea (Borg scale), discomfort (10-point scale), PaCO, , heart rate, blood
pressure, $\mathrm{SpO}_{2}$, global rating, patient preference

Note: we did not include outcome data in the review because the study authors did not report outcome data from the first study period.

Notes

Funding/declarations of interest: Fisher \& Paykel Healthcare Ltd provided 2 Optiflow devices at no charge for the study. Investigators received no financial support and manufacturer had no part in study design, conduct, analysis, reporting, or publication.

Study dates: March 2009 to March 2011 
Shebl 2018

\section{Study characteristics}

\begin{tabular}{|c|c|}
\hline Methods & RCT, parallel-group design. Single-centre study \\
\hline Participants & 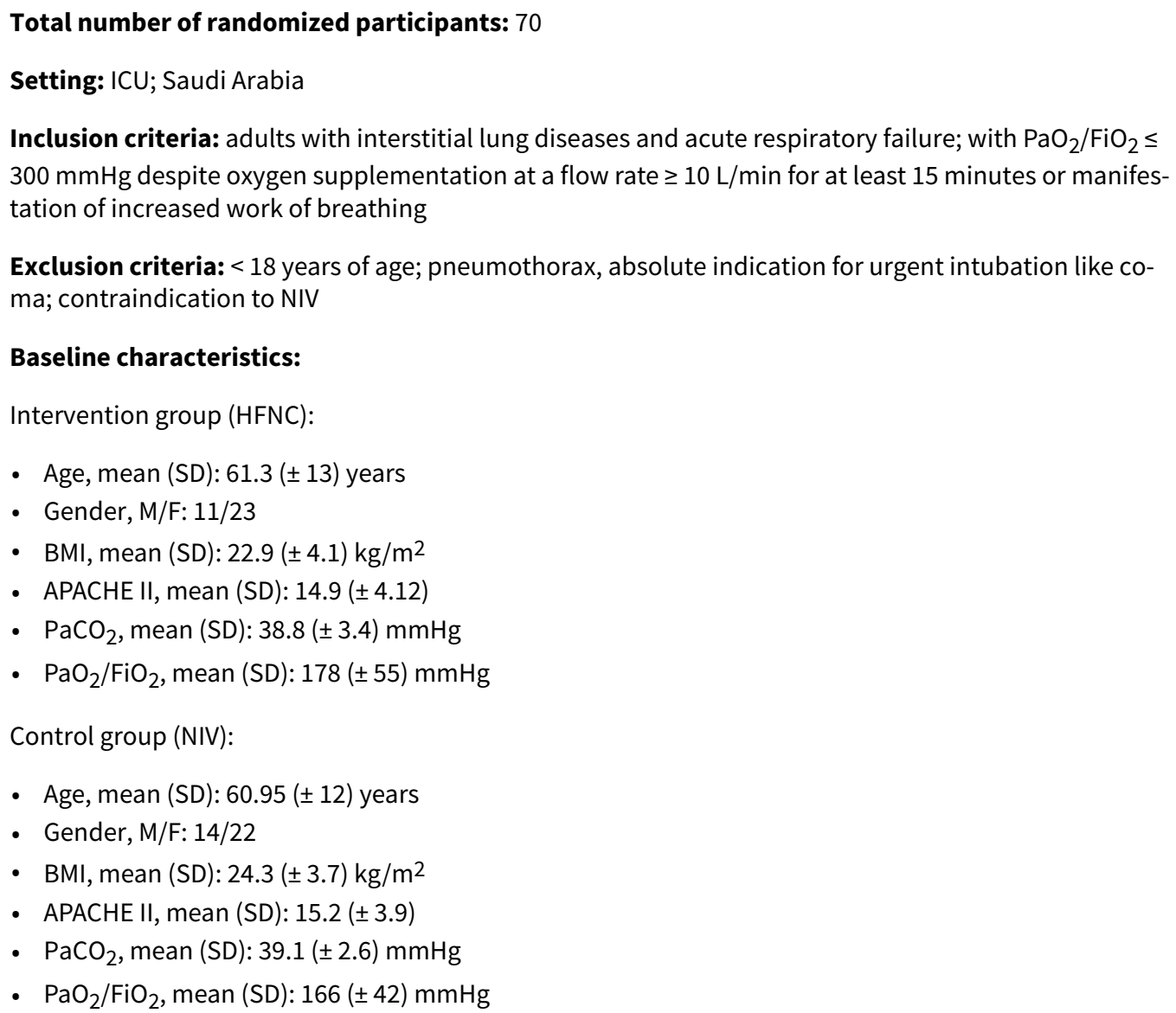 \\
\hline
\end{tabular}

Interventions

Intervention group (HFNC):

- Randomized, $\mathrm{n}=34$; losses, $\mathrm{n}=0$; analysed, $\mathrm{n}=34$

- Details: oxygen delivered via Optiflow, using a large-diameter nasal cannula. Therapy until the participant recovered or was intubated

Control group (NIV):

- Randomized, $\mathrm{n}=36$; losses, $\mathrm{n}=0$; analysed, $\mathrm{n}=36$

- Details: NIV using BiPAP Vision. Continuous positive airway pressure mode initiated for NIV, gradually incremented to $12 \mathrm{~cm} \mathrm{H}_{2} \mathrm{O}$. Pressure support for respiratory acidosis of if respiratory rate $>30$ breaths/ min. $\mathrm{FiO}_{2}$ adjusted at lowest level to maintain $\mathrm{PaO}_{2}>60 \mathrm{mmHg}$

Study dates: January 2016 to May 2017

\section{Risk of bias}


Shebl 2018 (Continued)

Random sequence genera- Unclear risk Described as randomized, but no additional details tion (selection bias)

\begin{tabular}{lll}
\hline $\begin{array}{l}\text { Allocation concealment } \\
\text { (selection bias) }\end{array}$ & Unclear risk details
\end{tabular}

\section{Blinding of participants Unclear risk} and personnel (performance bias)

All outcomes

\begin{tabular}{lll}
\hline $\begin{array}{l}\text { Blinding of outcome as- } \\
\text { sessors (objective out- } \\
\text { comes) }\end{array}$ & Low risk & $\begin{array}{l}\text { Blinding of outcome assessors was not described; we did not anticipate that } \\
\text { this would influence the assessment of objective outcome measures. }\end{array}$ \\
\hline $\begin{array}{l}\text { Incomplete outcome data } \\
\text { (attrition bias) } \\
\text { All outcomes }\end{array}$ & Low risk & No losses \\
\hline $\begin{array}{l}\text { Selective reporting (re- } \\
\text { porting bias) }\end{array}$ & Unclear risk & $\begin{array}{l}\text { Study authors did not report clinical trials registration or prepublished proto- } \\
\text { col. It was not feasible to effectively assess risk of selective reporting bias with- } \\
\text { out these documents. }\end{array}$ \\
\hline Other bias & Low risk & We identified no other sources of bias. \\
\hline
\end{tabular}
outcome data, we could not be certain of this.
Blinding was not possible. Although we expected that this would not influence

\section{Song 2017}

\section{Study characteristics}

\begin{tabular}{|c|c|}
\hline Methods & $\mathrm{RCT}$, parallel-group design; single-centre study \\
\hline Participants & 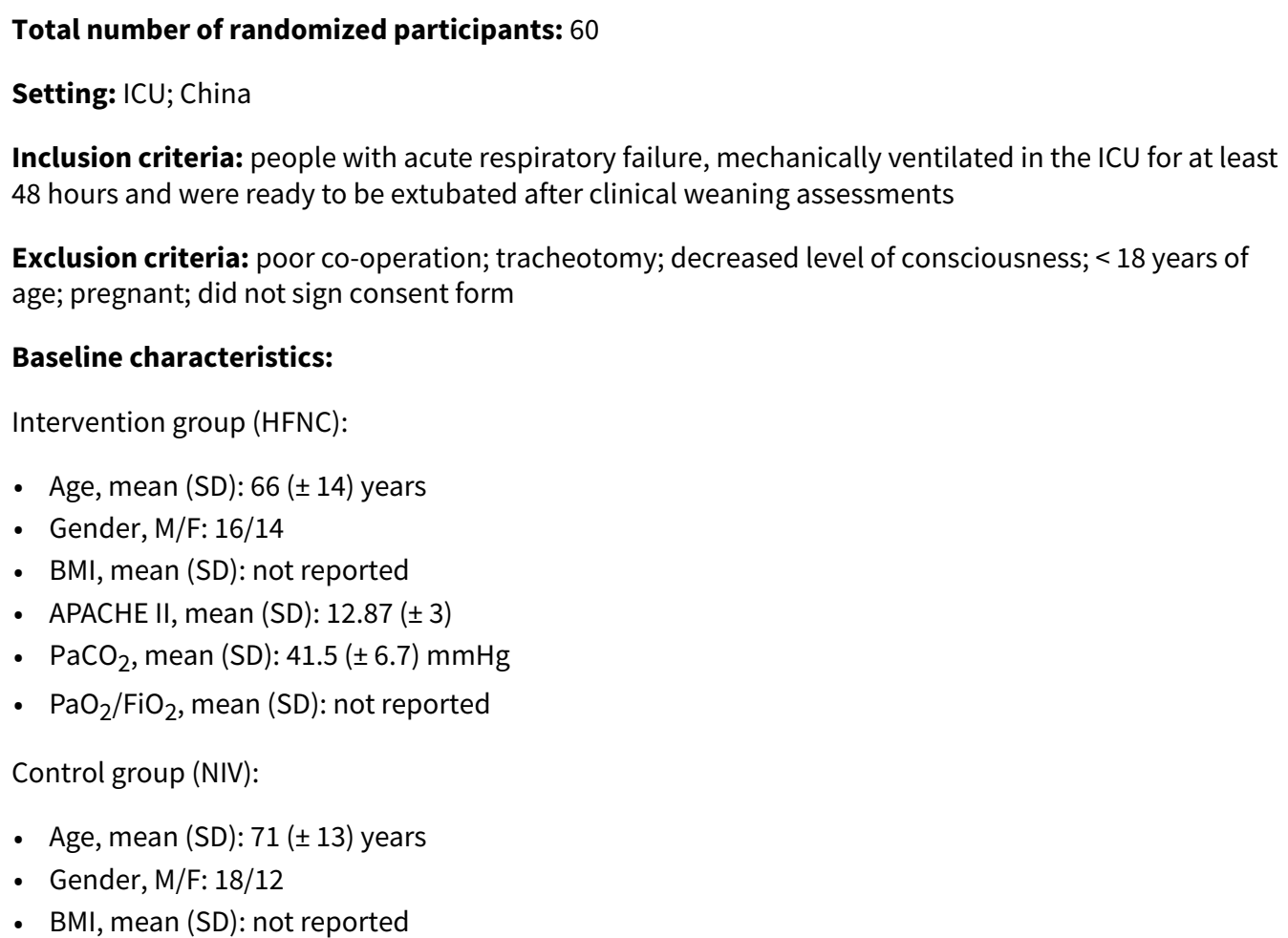 \\
\hline
\end{tabular}


Song 2017 (Continued)

- APACHE II, mean (SD): $12.36( \pm 3.29)$

- $\mathrm{PaCO}_{2}$, mean (SD): $42.3( \pm 7.1) \mathrm{mmHg}$

- $\mathrm{PaO}_{2} / \mathrm{FiO}_{2}$, mean (SD): not reported

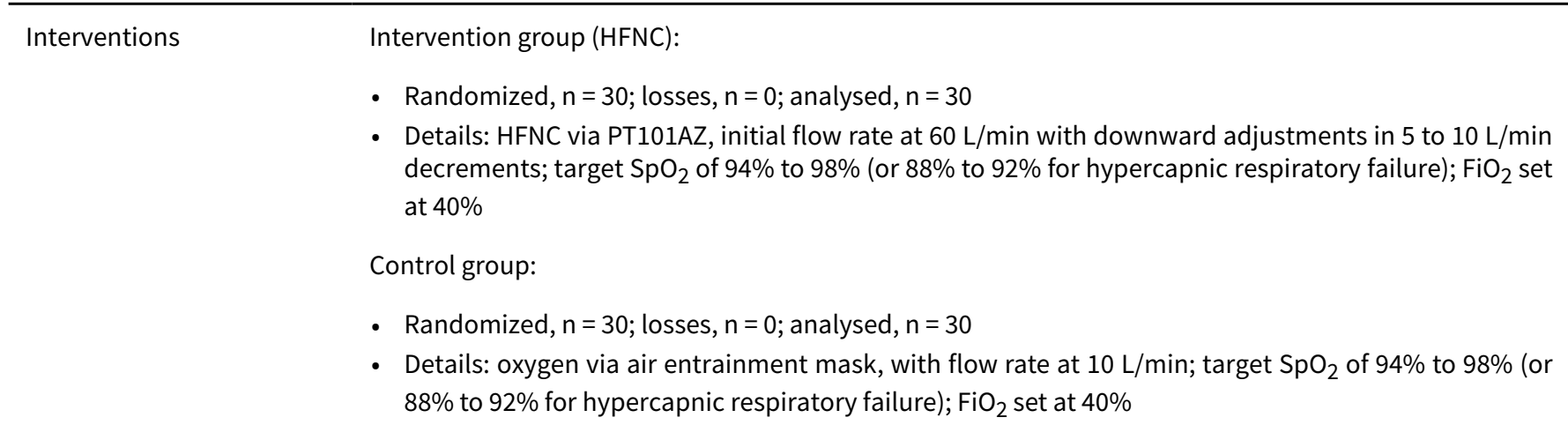
Outcomes
Success of oxygen therapy; needing NIV, or MV, or replacement of oxygen device; respiratory variables $\left(\mathrm{PaO}_{2} ; \mathrm{SpO}_{2} ; \mathrm{PaCO}_{2}\right.$; respiratory rate): haemodynamic variables; discomfort (scale 0 to $10=$ no discom- fort to maximum discomfort)

Notes

Funding/declarations of interest: funded by grants from the National Natural Science Foundation of China, the Medical and Health Research Program of Zhejiang Province, and the Medical and Health Research Program of Zheijing Province

Study dates: January 2013 to December 2014

\section{Risk of bias}

\begin{tabular}{|c|c|c|}
\hline Bias & Authors' judgement & Support for judgement \\
\hline $\begin{array}{l}\text { Random sequence genera- } \\
\text { tion (selection bias) }\end{array}$ & Low risk & Computer-generated randomization \\
\hline $\begin{array}{l}\text { Allocation concealment } \\
\text { (selection bias) }\end{array}$ & Unclear risk & No details \\
\hline $\begin{array}{l}\text { Blinding of participants } \\
\text { and personnel (perfor- } \\
\text { mance bias) } \\
\text { All outcomes }\end{array}$ & Unclear risk & $\begin{array}{l}\text { Blinding was not possible. Although we expected that this would not influence } \\
\text { outcome data, we could not be certain of this. }\end{array}$ \\
\hline $\begin{array}{l}\text { Blinding of outcome as- } \\
\text { sessors (objective out- } \\
\text { comes) }\end{array}$ & Low risk & $\begin{array}{l}\text { Blinding of outcome assessors was not described; we did not anticipate that } \\
\text { this would influence the assessment of objective outcome measures. }\end{array}$ \\
\hline $\begin{array}{l}\text { Blinding of outcome as- } \\
\text { sessors (subjective mea- } \\
\text { sures) }\end{array}$ & Low risk & $\begin{array}{l}\text { Participants were outcome assessors for discomfort using a standardized } \\
\text { scale; we did not expect that this would influence the outcome data. }\end{array}$ \\
\hline $\begin{array}{l}\text { Incomplete outcome data } \\
\text { (attrition bias) } \\
\text { All outcomes }\end{array}$ & Low risk & No apparent losses \\
\hline $\begin{array}{l}\text { Selective reporting (re- } \\
\text { porting bias) }\end{array}$ & Unclear risk & $\begin{array}{l}\text { Study authors did not report clinical trials registration or prepublished proto- } \\
\text { col. It was not feasible to effectively assess risk of selective reporting bias with- } \\
\text { out these documents. }\end{array}$ \\
\hline
\end{tabular}


Song 2017 (Continued)
Other bias
Low risk
We identified no other sources of bias.

Stephan 2015

\section{Study characteristics}

\section{Methods}

Participants
RCT, parallel-group design, multicentre study

\section{Total number of randomized participants: 830}

Setting: 6 ICUs; France

Inclusion criteria: patients who had undergone cardiothoracic surgery and had failed a SBT, or had pre-existing risk factors for post-extubation acute respiratory failure, or had failed extubation

Exclusion criteria: obstructive sleep apnoea, tracheostomy, do-not-intubate status, delirium, nausea and vomiting, bradypnoea, impaired consciousness, haemodynamic instability

\section{Baseline characteristics}

Intervention group (HFNC):

- Age, mean ( $95 \% \mathrm{Cl}): 63.8$ (62.5 to 65.2 ) years

- Gender M/F: 273/141

- BMI, mean (95\% Cl): 28.3 (27.8 to 28.8$) \mathrm{kg} / \mathrm{m}^{2}$

- SAPS II, mean (95\% Cl): 29.0 (27.8 to 30.1 )

- $\mathrm{PaCO}_{2}$, mean $(95 \% \mathrm{Cl}): 38.7$ (38.1 to 39.4$) \mathrm{mmHg}$

- $\mathrm{PaO}_{2} / \mathrm{FiO}_{2}$, mean (95\% Cl): 196 (187 to 204) mmHg

- Respiratory rate, mean ( $95 \% \mathrm{Cl}$ ): 22.8 (22.1 to 23.5 ) breaths/min

Control group (BiPAP):

- Age, mean ( $95 \% \mathrm{Cl}): 63.9$ (62.6 to 65.2 ) years

- Gender, M/F: 278/138

- BMI, mean $(95 \% \mathrm{CI}): 28.2$ (27.6 to 28.7$) \mathrm{kg} / \mathrm{m}^{2}$

- SAPS II, mean (95\% Cl): 28.8 (27.7 to 30.0$)$

- $\mathrm{PaCO}_{2}$, mean $(95 \% \mathrm{Cl}): 39.1$ (38.4 to 39.8$) \mathrm{mmHg}$

- $\mathrm{PaO}_{2} / \mathrm{FiO}_{2}$, mean (95\% Cl): 203 (195 to 212 ) mmHg

- Respiratory rate, mean ( $95 \% \mathrm{CI}): 23.3$ (22.6 to 24.0 ) breaths/min
Intervention group (HFNC):

- Randomized, $n=414$; losses, $n=0$; analysed, $n=414$

- Details: HFNC; Optiflow system at initial flow rate of $50 \mathrm{~L} / \mathrm{min}$. Delivered continuously

Control group (NIV: BiPAP):

- Randomized, $\mathrm{n}=416$; losses, $\mathrm{n}=0$; analysed, $\mathrm{n}=416$

- Details: BiPAP; pressure support started at $8 \mathrm{~cm} \mathrm{H}_{2} \mathrm{O}$ to achieve exhaled tidal volume of $8 \mathrm{~mL} / \mathrm{kg}$ and respiratory rate $<25$ breaths per minute, via full face mask and ventilator specifically designed for BiPAP or an ICU ventilator. BiPAP was delivered for 2 hours initially, then for approximately 1 hour every 4 hours, or more if needed.

Initial $\mathrm{FiO}_{2}$ in both groups was $50 \%$, adjusted to maintain $\mathrm{SaO}_{2}$ at $92 \%$ to $98 \%$ 
Stephan 2015 (Continued)

Outcomes
Treatment failure (defined as reintubation for MV, switch to other study treatment, or premature study treatment discontinuation), duration of respiratory support, respiratory variables, dyspnoea, comfort, skin breakdown, respiratory and extrapulmonary complications, number of bronchoscopies, mortality in ICU

\section{Note:}

- respiratory variables and respiratory rate reported at baseline, 1 hour, and 6 to 12 hours. For metaanalysis in the review, data were taken at 6 to 12 hours.

- we did not include data for duration of respiratory support because of differences in method of use, with HFNC used continuously and BiPAP used for approximately one hour at four-hourly windows.

\section{Notes} no conflicts of interest.

Study dates: June 2011 to January 2014

\section{Risk of bias}

\begin{tabular}{|c|c|c|}
\hline Bias & Authors' judgement & Support for judgement \\
\hline $\begin{array}{l}\text { Random sequence genera- } \\
\text { tion (selection bias) }\end{array}$ & Low risk & Computer-generated random sequence in blocks of 2 or 4 \\
\hline $\begin{array}{l}\text { Allocation concealment } \\
\text { (selection bias) }\end{array}$ & Unclear risk & Use of opaque envelopes but no further details \\
\hline $\begin{array}{l}\text { Blinding of participants } \\
\text { and personnel (perfor- } \\
\text { mance bias) } \\
\text { All outcomes }\end{array}$ & Unclear risk & $\begin{array}{l}\text { Blinding was not possible. Although we expected that this would not influence } \\
\text { outcome data, we could not be certain of this. }\end{array}$ \\
\hline $\begin{array}{l}\text { Blinding of outcome as- } \\
\text { sessors (objective out- } \\
\text { comes) }\end{array}$ & Low risk & $\begin{array}{l}\text { Blinding of outcome assessors was not described; we did not anticipate that } \\
\text { this would influence the assessment of objective outcome measures. }\end{array}$ \\
\hline $\begin{array}{l}\text { Blinding of outcome as- } \\
\text { sessors (subjective mea- } \\
\text { sures) }\end{array}$ & Low risk & $\begin{array}{l}\text { Participants were outcome assessors for dyspnoea and comfort; we did not ex- } \\
\text { pect this to influence the outcome data. We did not know if outcome assessors } \\
\text { for skin breakdown and other complications were blinded, however, complica- } \\
\text { tions were predefined to reduce bias. }\end{array}$ \\
\hline $\begin{array}{l}\text { Incomplete outcome data } \\
\text { (attrition bias) } \\
\text { All outcomes }\end{array}$ & Low risk & No losses \\
\hline $\begin{array}{l}\text { Selective reporting (re- } \\
\text { porting bias) }\end{array}$ & Low risk & $\begin{array}{l}\text { NCT01458444. Study registered retrospectively in October } 2011 \text { (although ear- } \\
\text { ly in study period). All relevant outcomes were reported as stated in protocol. }\end{array}$ \\
\hline Other bias & Low risk & No other sources of bias identified \\
\hline
\end{tabular}

Vargas 2015

\section{Study characteristics}

Methods RCT, cross-over design. Single-centre study


Vargas 2015 (Continued)

Participants
Total number of randomized participants: 12

Setting: ICU; France

Inclusion criteria: people with AHRF

Exclusion criteria: people with tracheostomies; < 18 years of age; chronic retention of $\mathrm{CO}_{2}$; respiratory acidosis; factors related to insertion of an oesophageal catheter; excessive amounts of respiratory secretions; SBP < 90 mmHg; ventricular arrhythmia; encephalopathy or coma; life-threatening hypoxaemia; decision to limit life-support treatments in the ICU

\section{Baseline characteristics (overall):}

- Age, median (IQR): 63 (59 to 73) years

- Gender, M/F: 10/2

- BMI, mean (SD): not reported

- SAPS II, median (IQR): 48 (35 to 56)

- $\mathrm{PaCO}_{2}$ : not reported

- $\mathrm{PaO}_{2} / \mathrm{FiO}_{2}$, median (IQR): 178 (157 to 199$) \mathrm{mmHg}$
Cross-over study with each period lasting approximately 20 minutes. All participants were first given conventional oxygen therapy using a non-rebreather face mask; participants were not randomized to this group.

Intervention group (HFNC):

- Randomized, $\mathrm{n}=12 ;$ losses, $\mathrm{n}=0$; analysed, $\mathrm{n}=12$

- Details: HFNC via Optiflow, with largest cannula tolerated by the individual participants, gas flow set at $60 \mathrm{~L} / \mathrm{min}$, temperature set at $37^{\circ} \mathrm{C}$

Control group (NIV: BiPAP):

- Randomized, $\mathrm{n}=12$; losses, $\mathrm{n}=0$; analysed, $\mathrm{n}=12$

- Details: BiPAP fitted to a face mask. CPAP set at $5 \mathrm{~cm} \mathrm{H}_{2} \mathrm{O}$
Oesophageal pressure; blood gas analysis and haemodynamic variables; comfort and dyspnoea

Note: we did not include outcome data in the review because the study authors did not report outcome data from the first study period.

Study dates: January 2011 to January 2012

\section{Study characteristics}

\begin{tabular}{ll}
\hline Methods & RCT, parallel-group design. Single-centre study \\
\hline Participants & Total number of randomized participants: 98 \\
& Setting: ICU; France \\
& $\begin{array}{l}\text { Inclusion criteria: } \geq 18 \text { years of age; admitted to the ICU after CABG presenting with severe hypox- } \\
\text { aemia after extubation }\end{array}$
\end{tabular}


Exclusion criteria: pregnancy; chronic respiratory failure; combined cardiac surgery; alteration of consciousness or requiring immediate intubation; surgical complications requiring re-operation; haemodynamic instability or ventricular arrhythmia; adults subject to legal protection; already participating in an interventional study on oxygenation

\section{Baseline characteristics:}

Intervention group (HFNC):

- Age, mean (SD): $67.6( \pm 9.4)$ years

- Gender, M/F: 36/7

- BMI, mean (SD): $28.7( \pm 3.8) \mathrm{kg} / \mathrm{m}^{2}$

- SAPS II, mean (SD): $26.9( \pm 9.4)$

- $\mathrm{PaCO}_{2}$, mean (SD): $39.8( \pm 4.5) \mathrm{mmHg}$

- $\mathrm{PaO}_{2} / \mathrm{FiO}_{2}$, mean (SD): $131.5( \pm 27.7) \mathrm{mmHg}$

Control group:

- Age, mean (SD): $65.8( \pm 10.1)$ years

- Gender, M/F: 41/8

- BMI, mean (SD): $29.7(4.5) \mathrm{kg} / \mathrm{m}^{2}$

- SAPS II, mean (SD): $26.4( \pm 6.0)$

- $\mathrm{PaCO}_{2}$, mean (SD): $40.5( \pm 3.8) \mathrm{mmHg}$

- $\mathrm{PaO}_{2} / \mathrm{FiO}_{2}$, mean (SD): $147.7( \pm 30.7) \mathrm{mmHg}$

Interventions

Intervention group (HFNC):

- Randomized, $\mathrm{n}=49$; losses, $\mathrm{n}=8$ ( 2 withdrew consent; 6 not included in per protocol analysis: 1 without severe hypoxaemia, 4 received control group therapy, 1 intubation before day 1 ); analysed, $n=47$ in ITT; 41 in per protocol

- Details: after extubation, HFNC via Optiflow, with gas flow rate of $45 \mathrm{~L} / \mathrm{min}, \mathrm{FiO}_{2}$ of $100 \%$ and adjusted according to the $\mathrm{SpO}_{2}$, and temperature of $37^{\circ} \mathrm{C}$. Device was switched every 6 hours to a Venturi mask to avoid hyperoxia.

Control group:

- Randomized, $n=49$; losses, $n=8$ ( 6 withdrew consent; 2 not included in per protocol analysis: 1 without severe hypoxaemia; 1 received intervention group therapy); analysed, $n=43$ in ITT, 41 in per protocol

- Details: non-rebreather facemask (Hudson $\mathrm{RCl}$ ) with humidified oxygen, gas flow rate of $15 \mathrm{~L} / \mathrm{min}$, with $\mathrm{FiO}_{2}$ of $100 \%$ and adjusted according to the $\mathrm{SpO}_{2}$. No CPAP valve on the face mask. Device was switched every 6 hours to a Venturi mask to avoid hyperoxia.

Outcomes $\mathrm{PaO}_{2} / \mathrm{FiO}_{2}$ (1, 6, 24 and 48 hours); $\mathrm{PACO}_{2}$, respiratory rate and heart rate at 48 hours; treatment failure defined as $\mathrm{SpO}_{2}<96 \%$ despite treatment or respiratory rate $\geq 25$ breaths/min; need for NIV or reintubation for treatment failure; increased work of breathing, or hypercapnia; tolerance of the device (satisfaction; occurrence of nasal bleeding; mucus dryness during therapy); radiologic score on chest X-ray; mortality; length of stay in the ICU

Note: data were available as ITT and per protocol. We used the ITT data because these data were clearly reported by study authors.

Notes

Funding/declarations of interest: source of funding (a grant for research and innovation missions) was not specified. However, funding was allocated to the university sponsor and Fischer \& Paykel; Fischer \& Paykel did not participate in study design, conduct, data management or interpretation of the results. Individual authors declared personal fees and funding from LFB, Fischer \& Paykel, Baxter, MSD, and Pfizer for other work. 
Vourc'h 2020 (Continued)

Study dates: June 2011 to April 2015

\section{Risk of bias}

\begin{tabular}{|c|c|c|}
\hline Bias & Authors' judgement & Support for judgement \\
\hline $\begin{array}{l}\text { Random sequence genera- } \\
\text { tion (selection bias) }\end{array}$ & Low risk & Computer-generated randomization \\
\hline $\begin{array}{l}\text { Allocation concealment } \\
\text { (selection bias) }\end{array}$ & Low risk & $\begin{array}{l}\text { Randomization controlled by an independent research unit, allocation provid- } \\
\text { ed via opaque envelopes }\end{array}$ \\
\hline $\begin{array}{l}\text { Blinding of participants } \\
\text { and personnel (perfor- } \\
\text { mance bias) } \\
\text { All outcomes }\end{array}$ & Unclear risk & $\begin{array}{l}\text { Blinding was not possible. Although we expected that this would not influence } \\
\text { outcome data, we could not be certain of this. }\end{array}$ \\
\hline $\begin{array}{l}\text { Blinding of outcome as- } \\
\text { sessors (objective out- } \\
\text { comes) }\end{array}$ & Low risk & $\begin{array}{l}\text { Blinding of outcome assessors was not described; we did not anticipate that } \\
\text { this would influence the assessment of objective outcome measures. }\end{array}$ \\
\hline $\begin{array}{l}\text { Blinding of outcome as- } \\
\text { sessors (subjective mea- } \\
\text { sures) }\end{array}$ & Low risk & $\begin{array}{l}\text { Study was described as open-label, and we assumed that participants were } \\
\text { aware of their group allocation. We did not think that lack of blinding would in- } \\
\text { fluence participants' assessment of subjective measures. }\end{array}$ \\
\hline $\begin{array}{l}\text { Incomplete outcome data } \\
\text { (attrition bias) } \\
\text { All outcomes }\end{array}$ & Low risk & $\begin{array}{l}\text { We noted losses in each group, however, these were clearly reported and rea- } \\
\text { sonably balanced and we did not expect the losses to cause risk of attrition } \\
\text { bias. }\end{array}$ \\
\hline $\begin{array}{l}\text { Selective reporting (re- } \\
\text { porting bias) }\end{array}$ & Unclear risk & $\begin{array}{l}\text { Registration with a clinical trials register, or a prepublished protocol was not } \\
\text { reported. It was not feasible to effectively assess risk of selective reporting bias } \\
\text { without these reports. }\end{array}$ \\
\hline Other bias & Low risk & We identified no other sources of bias. \\
\hline
\end{tabular}

Yu 2017

\section{Study characteristics}

\begin{tabular}{ll} 
Methods & RCT, parallel-group design. Multicentre study \\
\hline
\end{tabular}

Participants

\section{Total number of randomized participants: 110}

Setting: ICUs in 3 hospitals; China

Inclusion criteria: undergoing planned thoracoscopic lobectomy because of lung tumour, and at intermediate or high risk for postoperative pulmonary complications

Exclusion criteria: immunocompromised; pregnant; converted to an open thoracotomy because of poor visualization or bleeding; $<18$ or $>80$ years of age; informed consent could not be obtained

\section{Baseline characteristics:}

Intervention group (HFNC):

- Age, mean (SD): $56.31( \pm 7.03)$ years

- Gender, M/F: 30/26 
Yu 2017 (Continued)

- BMI, mean (SD): $26.32( \pm 4.73) \mathrm{kg} / \mathrm{m}^{2}$

- APACHE II, mean (SD): $26.32( \pm 4.73)$

- $\mathrm{PaCO}_{2}$, mean (SD): $41.73( \pm 6.33) \mathrm{mmHg}$

- $\mathrm{PaO}_{2} / \mathrm{FiO}_{2}$, mean (SD): $350.35( \pm 33.87) \mathrm{mmHg}$

- Respiratory rate, mean (SD): 18.43 ( \pm 3.45 ) breaths/min

Control group (standard oxygen therapy):

- Age, mean (SD): $55.82( \pm 7.92)$ years

- Gender, M/F: 28/26

- BMI, mean (SD): $25.19( \pm 5.02) \mathrm{kg} / \mathrm{m}^{2}$

- APACHE II, mean (SD): 25.19 ( \pm 5.02$)$

- $\mathrm{PaCO}_{2}$, mean (SD): $43.52( \pm 4.93) \mathrm{mmHg}$

- $\mathrm{PaO}_{2} / \mathrm{FiO}_{2}$, mean (SD): $340.98( \pm 40.65) \mathrm{mmHg}$

- Respiratory rate, mean (SD): $17.98( \pm 3.87)$ breaths/min

Interventions Intervention group (HFNC):

- Randomized, $\mathrm{n}=56$; losses, $\mathrm{n}=0$; analysed, $\mathrm{n}=56$

- Details: after extubation, after tolerating SBT, HFNC delivered by Optiflow (using MR850 heated humidifier and RT202 breathing circuit) with flow rate of 35 to $60 \mathrm{~L} / \mathrm{min}, \mathrm{FiO}_{2}$ titrated by treating clinician to maintain $\mathrm{SpO}^{2} \geq 95 \%$

Control group:

- Randomized, $\mathrm{n}=54$; losses, $\mathrm{n}=0$; analysed, $\mathrm{n}=54$

- Details: after extubation, after tolerating SBT, oxygen delivered via nasal prongs or facemask with oxygen flow titrated by treating clinician to maintain $\mathrm{SpO}_{2} \geq 95 \%$

Outcomes

Incidence of hypoxaemia in first 72 hours after extubation; $\mathrm{PaO}_{2} ; \mathrm{PaO}_{2} / \mathrm{FiO}_{2}, \mathrm{SpO}_{2} / \mathrm{FiO}_{2}$, and $\mathrm{PaCO}_{2}$; postoperative pulmonary complications (pneumonia and atelectasis); AHRF (for which participants were initially given NIV with BiPAP and, if required, were then reintubated); adverse effects (air leak, throat or nasal pain, abdominal distension); mortality; length of ICU and hospital stay; total hospitalization expenditure

Note: we did not include data for arterial gases in the review, because study authors presented these data in figures that we could not clearly interpret as numerical data.

Notes

Funding/declarations of interest: funding not reported. Study authors declared no competing interests.

Study dates: January 2015 to June 2016

\section{Risk of bias}

\begin{tabular}{lll}
\hline Bias & Authors' judgement & Support for judgement \\
\hline $\begin{array}{l}\text { Random sequence genera- } \\
\text { tion (selection bias) }\end{array}$ & Low risk & Randomization using STATA statistical package \\
\hline $\begin{array}{l}\text { Allocation concealment } \\
\text { (selection bias) }\end{array}$ & Unclear risk & No details \\
\hline
\end{tabular}

Blinding of participants Unclear risk and personnel (performance bias)

All outcomes
Blinding was not possible. Although we expected that this would not influence outcome data, we could not be certain of this. 
Yu 2017 (Continued)

Blinding of outcome as- Low risk Blinding of outcome assessors was not described; we did not anticipate that sessors (objective outcomes) this would influence the assessment of objective outcome measures.

\begin{tabular}{|c|c|c|}
\hline $\begin{array}{l}\text { Blinding of outcome as- } \\
\text { sessors (subjective mea- }\end{array}$ & Low risk & $\begin{array}{l}\text { Blinding of outcome assessors was not described; we did not anticipate that } \\
\text { this would influence the assessment of subjective outcome measures. }\end{array}$ \\
\hline
\end{tabular}
sures)

$\begin{aligned} & \text { Incomplete outcome data } \\ & \text { (attrition bias) }\end{aligned}$
Low risk participant losses

(attrition bias)

All outcomes

\begin{tabular}{|c|c|c|}
\hline $\begin{array}{l}\text { Selective reporting (re- } \\
\text { porting bias) }\end{array}$ & Unclear risk & $\begin{array}{l}\text { Study authors did not report clinical trials registration or a prepublished pro- } \\
\text { tocol. It was not feasible to effectively assess risk of selective reporting bias } \\
\text { without these documents. }\end{array}$ \\
\hline
\end{tabular}

Other bias Low risk We identified no other sources of bias.

Zochios 2018

\section{Study characteristics}

\begin{tabular}{|c|c|}
\hline Methods & RCT, parallel-group design. Single-centre study \\
\hline Participants & 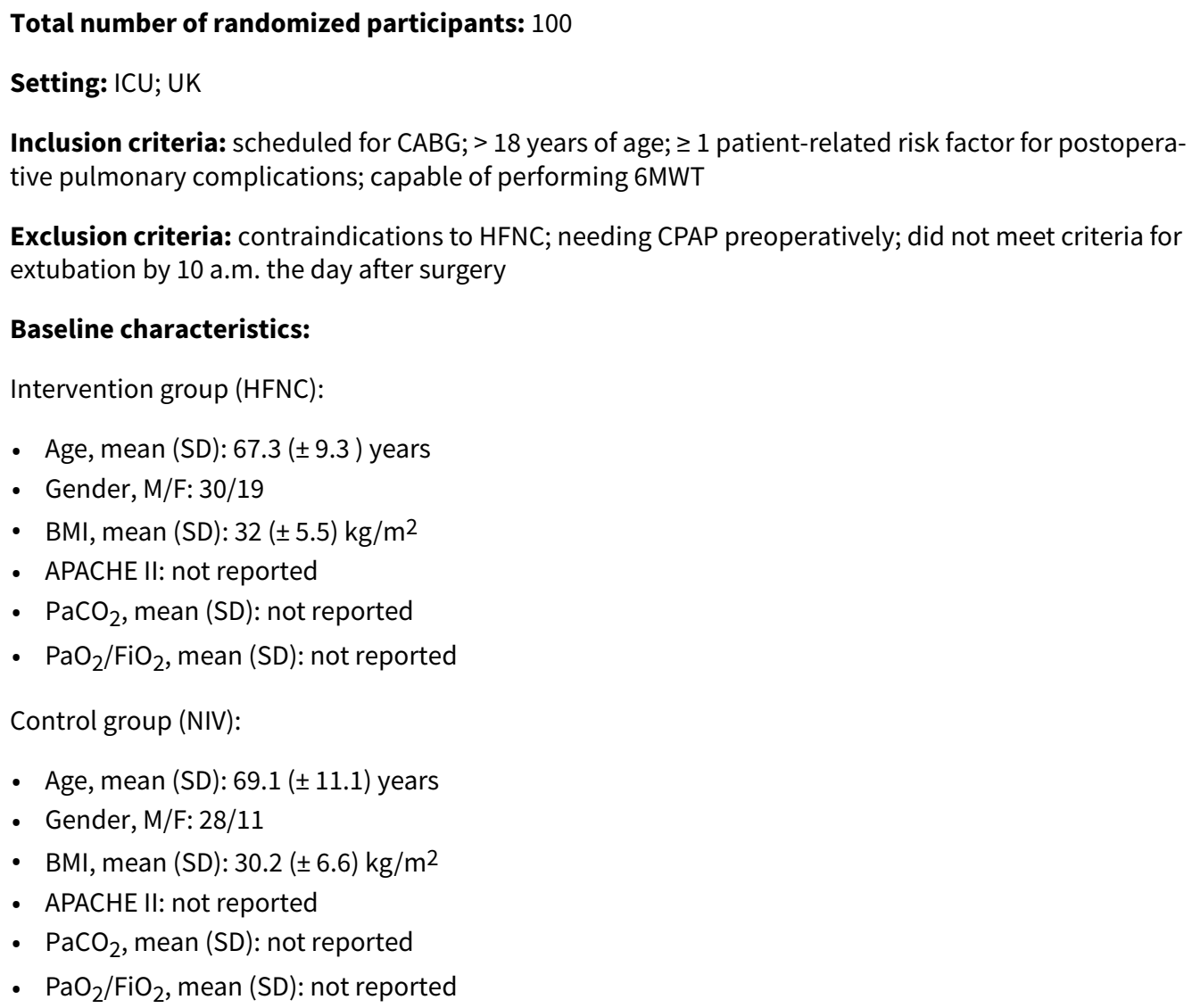 \\
\hline
\end{tabular}

Interventions

Intervention group: 
- Randomized, $\mathrm{n}=51$; losses, $\mathrm{n}=2$ (delayed extubation); analysed, $\mathrm{n}=49$

- Details: post-extubation in the ICU. $\mathrm{FiO}_{2}$ titrated to $\mathrm{SpO}_{2} \geq 95 \%$ (or $93 \%$ for those at risk of hypercapnic respiratory failure). Standard starting flow rate was $30 \mathrm{~L} / \mathrm{min}$, adjusted up or down within a range of 20 to $50 \mathrm{~L} / \mathrm{min}$ with aim of respiratory rate of $<16$ breaths/min and patient comfort. HFNO for 24 hours or longer if deemed necessary

Control group:

- Randomized, $n=49$; losses, $n=3$ ( 2 delayed extubation; 1 withdrew consent; procedure cancelled); analysed, $\mathrm{n}=45$

- Details: standard oxygen therapy via nasal prongs or soft facemask. $\mathrm{FiO}_{2}$ titrated to $\mathrm{SpO}_{2} \geq 95 \%$ (or $93 \%$ for those at risk of hypercapnic respiratory failure). Oxygen therapy for 24 hours or longer if deemed necessary

Outcomes Hospital length of stay; ICU length of stay; ICU re-admission rate; in-hospital mortality; escalation of respiratory support (unplanned CPAP, NIV or MV); pulmonary function tests; 6MWT; postoperative quality of recovery

Notes

Funding/declarations of interest: funded by AAGBI. Fisher and Paykel Healthcare Ltd provided equipment and were not involved in data collection, analysis, and writing the manuscript. One author has received educational funding, honoraria or travel assistance from CSL Behring, Massimo, Pharmacosmos, Fisher and Paykel, Brightwake Ltd and Vifor Pharma. No other declarations or competing interests declared

Study dates: not specified

\section{Risk of bias}

\begin{tabular}{|c|c|c|}
\hline Bias & Authors' judgement & Support for judgement \\
\hline $\begin{array}{l}\text { Random sequence genera- } \\
\text { tion (selection bias) }\end{array}$ & Low risk & Computer-generated randomization \\
\hline $\begin{array}{l}\text { Allocation concealment } \\
\text { (selection bias) }\end{array}$ & Low risk & Use of a centralised online system \\
\hline $\begin{array}{l}\text { Blinding of participants } \\
\text { and personnel (perfor- } \\
\text { mance bias) } \\
\text { All outcomes }\end{array}$ & Unclear risk & $\begin{array}{l}\text { Blinding was not possible. Although we expected that this would not influence } \\
\text { outcome data, we could not be certain of this. }\end{array}$ \\
\hline $\begin{array}{l}\text { Blinding of outcome as- } \\
\text { sessors (objective out- } \\
\text { comes) }\end{array}$ & Low risk & $\begin{array}{l}\text { Blinding of outcome assessors was not described; we did not anticipate that } \\
\text { this would influence the assessment of objective outcome measures. }\end{array}$ \\
\hline $\begin{array}{l}\text { Blinding of outcome as- } \\
\text { sessors (subjective mea- } \\
\text { sures) }\end{array}$ & Low risk & Staff responsible for decisions regarding patient care were blinded to groups. \\
\hline $\begin{array}{l}\text { Incomplete outcome data } \\
\text { (attrition bias) } \\
\text { All outcomes }\end{array}$ & Low risk & Few losses \\
\hline $\begin{array}{l}\text { Selective reporting (re- } \\
\text { porting bias) }\end{array}$ & High risk & $\begin{array}{l}\text { Study was prospectively registered with a clinical trials register } \\
\text { (NCT02496923). We noted that mortality was an additional outcome that was } \\
\text { not listed in the clinical trials register. All other outcomes were reported as de- } \\
\text { scribed in the register. }\end{array}$ \\
\hline Other bias & Low risk & We identified no other sources of bias. \\
\hline
\end{tabular}


AAGBI: Association of Anaesthetists of Great Britain and Ireland.

AHRF: acute hypoxaemic respiratory failure

AIDS: acquired immune deficiency syndrome

APACHE II: acute physiology and chronic health evaluation II

ASA: American Society of Anesthesiologists

BiPAP: bilevel positive airway pressure

BMI: body mass index

CABG: coronary artery bypass graft

$\mathrm{Cl}$ : confidence interval

COPD: chronic obstructive pulmonary disease

CPAP: continuous positive airway pressure

ECG: electrocardiographic

EIT: electrical impedance tomography

$\mathrm{FiO}_{2}$ : fraction of inspired oxygen

GCS: Glasgow coma score

HFFM: high-flow face mask

HFNC: high-flow nasal cannulae

HFNO: high-flow nasal oxygen

ICU: intensive care unit

IQR: interquartile range

ITT: intention-to-treat

MAP: mean arterial pressure

M/F: male/female

MV: mechanical ventilation

$\mathrm{n}$ : number of participants

NC: nasal cannula(e)

NIPPV: non-invasive positive-pressure ventilation

NIV: non-invasive ventilation

NIH: National Institutes of Health

NRS: numerical rating scale

$\mathrm{PaCO}_{2}$ : carbon dioxide clearance

$\mathrm{PaO}_{2}$ : partial pressure of arterial oxygen

PEEP: positive end-expiratory pressure

$\mathrm{pH}$ : potential of hydrogen

$\mathrm{SaO}_{2}$ : oxygen saturation of arterial blood

SAPS II: simplified acute physiological score

SBP: systolic blood pressure

SBT: spontaneous breathing trial

SD: standard deviation

SOFA: sequential organ failure assessment score

$\mathrm{SpO}_{2}$ : oxygen saturation

VAS: visual analogue scale

VPAP: this is the name of a device

6MWT: six minute walk test

Characteristics of excluded studies [ordered by study ID]

Study Reason for exclusion

Coudroy 2019

This was a multicentre RCT of adults with severe acute hypoxaemic respiratory failure in an ICU setting. Participants were randomized to receive either HFNC alone or HFNC and NIV. We excluded this study as HFNC were used in both study arms.

Delorme 2017

This was a cross-over RCT of adults with moderate respiratory distress in an ICU setting. Participants were given standard oxygen therapy up to baseline and then received HFNC with 3 different flow rates in a random order. We excluded this study as the control intervention (standard oxygen therapy) was delivered to all participants first, so this is not truly randomized. 


\begin{tabular}{ll}
\hline Study & Reason for exclusion \\
\hline Di Mussi 2016 & $\begin{array}{l}\text { This was a non-randomized cross-over study where participants were given HFNC, low-flow oxygen } \\
\text { and HFNC sequentially post-extubation in an ICU setting. We excluded this study as it was not ran- } \\
\text { domized. }\end{array}$ \\
\hline Lemiale 2016 & $\begin{array}{l}\text { This was a multicentre study of critically-ill immunocompromised adults receiving treatment for } \\
\text { haematological malignancies or solid tumours. Participants were randomized to receive either } \\
\text { NIV or oxygen therapy. Within the oxygen therapy group, the decision to use low-flow oxygen or } \\
\text { HFNC was at the discretion of the treating clinician. Therefore, randomization was not at the level } \\
\text { required for this review (i.e. HFNC vs low-flow oxygen therapy). }\end{array}$
\end{tabular}

Liu 2019 This was an RCT of weaning and post-extubation adults receiving invasive mechanical ventilation
in an ICU setting. Participants were randomized to receive T-tube, NIV or high-flow oxygen via their
endotracheal tube during a 2-hour spontaneous breathing trial. If they passed the SBT, participants
receiving T-tube or NIV were moved onto low-flow oxygen facemask whilst the high-flow oxygen
group were moved to HFNC. We excluded this study as the participants received different treat-
ment prior to the initiation of oxygen via HFNC or low-flow oxygen via facemask.

Pennisi 2019 This was an RCT of adults undergoing elective thoracotomic pulmonary lobar resection. Participants received oxygen either using HFNC or Venturi face mask. We excluded this study because very few participants received therapy in the ICU; most participants started therapy in the PACU before transfer to the surgical ward.

$\begin{array}{ll}\text { Sklar } 2018 & \text { This was a cross-over RCT of adults with cystic fibrosis with a clinical indication for NIV in a respira- } \\ \text { tory ward. Participants received standard oxygen up to baseline then HFNC and NIV in random or- } \\ \text { der. We excluded this study as participants were treated in a respiratory ward, not an ICU. }\end{array}$

Thille 2018

This was a multicentre RCT of adults post-extubation at high risk of post-extubation failure in an ICU setting. Participants were randomized to receive either HFNC alone or HFNC and NIV. We excluded this study as HFNC were used in both intervention arms.

Thille 2019

This was a multicentre RCT of adults post-extubation who were at high risk of post-extubation failure in an ICU setting. Participants were randomized to receive either HFNC alone or HFNC and NIV. We excluded this study as HFNC were used in both intervention arms.

HFNC: high-flow nasal cannula(e)

ICU: intensive care unit

NIV: non-invasive ventilation

PACU: post-anaesthesia care unit

$\mathrm{RCT}$ : randomized controlled trial

SBT: spontaneous breathing trial

Characteristics of studies awaiting classification [ordered by study ID]

Arman 2017

\begin{tabular}{ll}
\hline Methods & RCT, parallel-group design. Single-centre study \\
\hline Participants & Total number of randomized participants: 15 \\
& Setting: ICU, USA \\
& Inclusion criteria: intubated for Type I respiratory failure; $\mathrm{PaCO}_{2}<50 \mathrm{mmHg}$ \\
& Exclusion criteria: severe COPD \\
\hline
\end{tabular}

Interventions

Intervention group (HFNC); $\mathrm{n}=8$. 
Arman 2017 (Continued)

Control group (standard nasal oxygen); $\mathrm{n}=7$
All outcomes reported: oxygen flow; $\mathrm{FiO}_{2}$; oxygen saturation; respiratory rate; heart rate (all every $12 \mathrm{hrs}$ for $48 \mathrm{hrs}$ ); ABG 24 hrs post-extubation; reintubation; 30-day mortality

Outcomes relevant to this review: reintubation; 30-day mortality; oxygen saturation; respiratory rate; $A B G 24$ hours post-extubation
Contact: P.D. Arman, arman.pouyand@navicenthealth.org

Currently published only as an abstract. We are awaiting publication of the full report in order to assess eligibility, collect sufficient study characteristics, and include data in the review.

\section{Guoqiang 2018}

\begin{tabular}{ll}
\hline Methods & RCT, parallel-group design \\
\hline Participants & Total number of randomized participants: 36 \\
& Inclusion criteria: AECOPD; hypercapnia; ready for extubation \\
& Exclusion criteria: none reported \\
\hline Interventions & Intervention group (HFNC): $\mathrm{n}=19$ \\
& Control group (NIV): $\mathrm{n}=17$ \\
\hline Outcomes & $\begin{array}{l}\text { All outcomes reported: rate of treatment failure; reintubation rate; vital signs; ABG; comfort score; } \\
\text { bronchoscopy for secretion management within } 48 \text { hours }\end{array}$ \\
& $\begin{array}{l}\text { Outcomes relevant to this review: treatment failure; reintubation rate; vital signs; ABG; comfort } \\
\text { score }\end{array}$ \\
\hline Cotes & Contact: Jing Guoqiang, Binzhou Medical University, Binzhou, China \\
Currently published only as an abstract. We are awaiting publication of the full report in order to \\
assess eligibility, collect sufficient study characteristics, and include data in the review.
\end{tabular}

\section{Gupta 2016}

\begin{tabular}{|c|c|}
\hline Methods & RCT. parallel-group design. Single-centre study \\
\hline \multirow[t]{3}{*}{ Participants } & Number of participants: 20 \\
\hline & Inclusion criteria: postoperative liver transplant; respiratory failure \\
\hline & Exclusion criteria: none stated \\
\hline \multirow[t]{3}{*}{ Interventions } & Intervention group $(\mathrm{HFNC}): \mathrm{n}=10$; flow $=60 \mathrm{~L} / \mathrm{min}$; flow and $\mathrm{FiO}_{2}$ titrated to $\mathrm{ABG}$ \\
\hline & Control group (NIV): BiPAP; $n=10 ; \mathrm{PEEP}=5 \mathrm{~cm} \mathrm{H}_{2} \mathrm{O}$ and IPAP $=10 \mathrm{~cm} \mathrm{H}_{2} \mathrm{O}$; flow and $\mathrm{FiO}_{2}$ titrated to \\
\hline & ABG \\
\hline
\end{tabular}

Outcomes

All outcomes measured: ABG, comfort scale, RASS, CAM-ICU, nutritional deficit; intubation rate

Outcomes relevant to this review: intubation rate; $A B G$; comfort 
Gupta 2016 (Continued)

Notes
Contact: S. Gupta - Medanta - The Medicity, Gurgaon, India

Currently published only as an abstract. We are awaiting publication of the full report in order to assess eligibility, collect sufficient study characteristics, and include data in the review.

Ischaki 2019

\begin{tabular}{ll}
\hline Methods & RCT, parallel-group design \\
\hline Participants & $\begin{array}{l}\text { Total number of participants: } 27 \\
\text { Inclusion criteria: AECOPD and acute or acute on chronic hypercapnic respiratory failure; indication } \\
\text { for NIV } \\
\text { Exclusion criteria: none reported }\end{array}$ \\
\hline Interventions & $\begin{array}{l}\text { Intervention: HFNC } \\
\text { Comparator: NIV }\end{array}$ \\
\hline Outcomes & $\begin{array}{l}\text { All outcomes reported: treatment failure; changes in respiratory variables; patient comfort; pres- } \\
\text { ence of pulmonary and extrapulmonary complications }\end{array}$ \\
Outcomes relevant to this review: treatment failure; changes in respiratory variables; patient com- \\
fort; presence of pulmonary and extrapulmonary complications
\end{tabular}

\begin{tabular}{ll}
\hline Methods & RCT, parallel-group design. Single-centre study \\
\hline Participants & Number of participants: 94 \\
& Setting: post-surgical ICU, UK \\
& Inclusion criteria: elective cardiac surgery; age > $18 ; \geq 1$ risk factor for postoperative pulmonary \\
complications; able to perform 6 -minute walk test & $\begin{array}{l}\text { Exclusion criteria: contraindications to HFNC; extubation not met by } 10 \text { a.m. the day after surgery; } \\
\text { need for CPAP preoperatively }\end{array}$ \\
\hline Interventions & Intervention group (HFNC): $n=49 ;$ duration $\geq 24$ hrs \\
Control group (standard oxygen therapy): $n=45 ;$ soft face mask or nasal cannulae \\
\hline Outcomes \\
operative lung function; ICU length of stay; escalation of respiratory support and ICU re-admission \\
$\begin{array}{l}\text { Outcomes relevant to this review: hospital length of stay; ICU length of stay; escalation of respirato- } \\
\text { ry support }\end{array}$
\end{tabular}


The data from this study were available only via the trial registry, however, these findings have not been subject to peer review. We are awaiting publication of the full report in order to assess eligibility, collect sufficient study characteristics, and include data in the review.

\section{Lee 2016}

\begin{tabular}{ll}
\hline Methods & RCT, parallel-group design \\
\hline Participants & $\begin{array}{l}\text { Total number of participants: } 68 \\
\text { Inclusion criteria: severe acute exacerbation of COPD } \\
\text { Exclusion criteria: none reported }\end{array}$ \\
\hline Interventions & $\begin{array}{l}\text { Intervention: HFNC } \\
\text { Comparator: NIV }\end{array}$ \\
\hline Outcomes & $\begin{array}{l}\text { All outcomes reported: 30-day mortality; treatment failure (intubation) } \\
\text { Outcomes relevant to this review: 30-day mortality; treatment failure (intubation) }\end{array}$ \\
\hline Notes & $\begin{array}{l}\text { Contact: Myoung Kyu Lee, Department of Internal Medicine, Yonsei University Wonju College of } \\
\text { Medicine, Wonju, Republic of Korea }\end{array}$ \\
& $\begin{array}{l}\text { Currently published only as an abstract. We are awaiting publication of the full report in order to } \\
\text { assess eligibility, collect sufficient study characteristics, and include data in the review. }\end{array}$ \\
\hline
\end{tabular}

Longhini 2017

\begin{tabular}{ll}
\hline Methods & RCT, cross-over design \\
\hline Participants & Number of participants: 27. \\
& Inclusion criteria: acute on chronic respiratory failure; $\mathrm{pH}>7.34$; respiratory rate $\leq 30$ breaths/min \\
& Exclusion criteria: none in abstract \\
\hline Interventions & Intervention group (HFNC): flow = 60 L/min \\
& Control group: Venturi mask \\
\hline Outcomes & $\begin{array}{l}\text { All outcomes measured: ultrasound diaphragm displacement; diaphragm thickening fraction; dys- } \\
\text { pnoea; comfort; arterial blood gases }\end{array}$ \\
& Outcomes relevant to this review: dyspnoea; comfort; arterial blood gases \\
\hline Notes & $\begin{array}{l}\text { Contact: Federico Longhini, Anesthesia and Intensive Care, Sant'Andrea Hospital, ASL VC, Vercelli, } \\
\text { Italy } \\
\text { Currently published only as an abstract. We noted some similarities with Longhini 2019, but be- } \\
\text { cause of some variation in methodology, we have assumed this study to be separate. We are, there- } \\
\text { fore, awaiting publication of the full report in order to assess eligibility, collect sufficient study } \\
\text { characteristics, and include data in the review. }\end{array}$ \\
\hline
\end{tabular}


Macari 2019

\begin{tabular}{|c|c|}
\hline Methods & $\mathrm{RCT}$, cross-over design \\
\hline \multirow[t]{3}{*}{ Participants } & Total number of participants: not reported \\
\hline & Inclusion criteria: hypoxaemic ARF due to pneumonia; requiring HFNC and/or NIV \\
\hline & Exclusion criteria: cardiogenic pulmonary oedema; underlying respiratory disease \\
\hline \multirow[t]{2}{*}{ Interventions } & Intervention group (HFNC) \\
\hline & Control group (NIV) \\
\hline \multirow[t]{2}{*}{ Outcomes } & $\begin{array}{l}\text { All outcomes measured: global and regional end-expiratory electrical lung impedance; lung vol- } \\
\text { umes, respiratory parameters; haemodynamic tolerance; dyspnoea; comfort }\end{array}$ \\
\hline & Outcomes relevant to this review: respiratory parameters; dyspnoea; comfort \\
\hline \multirow[t]{2}{*}{ Notes } & Contact: Elise Artaud-Macari, eliseartaudmacari@yahoo.fr \\
\hline & $\begin{array}{l}\text { Currently published only as an abstract. We are awaiting publication of the full report in order to } \\
\text { assess eligibility, collect sufficient study characteristics, and include data in the review. }\end{array}$ \\
\hline
\end{tabular}

Menga 2019

\begin{tabular}{ll}
\hline Methods & RCT, cross-over design \\
\hline Participants & $\begin{array}{l}\text { Total number of participants: } 10 \\
\text { Inclusion criteria: acute-onset, non-cardiogenic respiratory distress; pulmonary infiltrates on the } \\
\text { chest x-ray; } \mathrm{SpO}_{2}<90 \% \\
\text { Exclusion criteria: none in abstract }\end{array}$ \\
\hline Interventions & $\begin{array}{l}\text { Intervention group (HFNC): flow }=50 \mathrm{~L} / \mathrm{min} \\
\text { Control group (helmet NIV): PEEP }=10 \mathrm{~cm} \mathrm{H}_{2} \mathrm{O} \text {. IPAP adjusted to achieve peak inspiratory flow }=100\end{array}$ \\
\hline Outcomes & $\begin{array}{l}\text { All outcomes measured: arterial blood gases, inspiratory effort (oesophageal pressure); respiratory } \\
\text { rate; dyspnoea; device-related discomfort }\end{array}$ \\
& $\begin{array}{l}\text { Outcomes relevant to this review: arterial blood gases; respiratory rate; dyspnoea; device-related } \\
\text { discomfort }\end{array}$ \\
\hline Notes & $\begin{array}{l}\text { Contact: LS Menga, Fondazione Policlinico Universitario A. Gemelli IRCCS, Department of Anesthe- } \\
\text { siology and Intensive Care Medicine, Rome, Italy } \\
\text { Currently published only as an abstract. We are awaiting publication of the full report in order to } \\
\text { assess eligibility, collect sufficient study characteristics, and include data in the review. }\end{array}$
\end{tabular}

\section{Papachatzakis 2017}

\begin{tabular}{ll}
\hline Methods & RCT, parallel-group design. Single-centre study \\
\hline Participants & Total number of participants: 20 \\
& Inclusion criteria: HDU, acute type II respiratory failure \\
& Exclusion criteria: none reported \\
\hline \hline
\end{tabular}


Papachatzakis 2017 (Continued)

$\begin{array}{ll}\text { Interventions } & \text { Control group (NIV, BiPAP): } \mathrm{n}=10\end{array}$

Outcomes

All outcomes reported: blood pressure; respiratory rate; pulse; $\mathrm{SpO}_{2} ;$ arterial $\mathrm{pH} ; \mathrm{PaO}_{2} ; \mathrm{PaCO}_{2}$; $\mathrm{HCO}_{3}$

Outcomes relevant to this review: respiratory rate; pulse; $\mathrm{SpO}_{2}$; arterial $\mathrm{pH} ; \mathrm{PaO}_{2} ; \mathrm{PaCO}_{2} ; \mathrm{HCO}_{3}$

Notes

Contact: Ioannis Papachatzakis, Department of Clinical Therapeutics, National and Kapodistrian University Medical School, Alexandra Hospital, Athens, Greece

Currently published only as an abstract. We are awaiting publication of the full report in order to assess eligibility, collect sufficient study characteristics, and include data in the review.

Perbet 2014

\begin{tabular}{ll}
\hline Methods & RCT. parallel-group design. Multicentre study \\
\hline Participants & Number of randomized participants: 80 \\
& Setting: four ICUs at 2 hospitals, France \\
& Inclusion criteria: mechanically ventilated patient ready for extubation \\
& Exclusion criteria: none reported \\
\hline Interventions & Intervention group (HFNC): $n=40$ \\
& Control group (standard oxygen therapy): $\mathrm{n}=40$ \\
Both for 48 hours post-extubation \\
\hline Autcomes \\
$\begin{array}{l}\text { All outcomes reported: lung ultrasound score, dyspnoea, post-extubation distress incidence; treat- } \\
\text { ICU and hospital mortalities }\end{array}$ \\
$\begin{array}{l}\text { Outcomes relevant to this review: treatment failure rate; clinical respiratory variables; hospital } \\
\text { mortality; dyspnoea }\end{array}$ \\
$\begin{array}{l}\text { Contact: S. Perbet, University Hospital of Clermont-Ferrand, ICU, Clermont-Ferrand, France } \\
\text { Currently published only as an abstract. We are awaiting publication of the full report in order to } \\
\text { assess eligibility, collect sufficient study characteristics, and include data in the review. }\end{array}$ \\
\hline Notes
\end{tabular}

Saeed 2015

\begin{tabular}{ll}
\hline Methods & Not stated if this was an RCT, parallel-group design. Single-centre study \\
\hline Participants & Total number of participants: 85 \\
& Setting: respiratory ICU, Egypt \\
& Inclusion criteria: COPD; type II respiratory failure; admitted to respiratory ICU \\
& Exclusion criteria: none reported \\
\hline Interventions & Intervention group (HFNC): $\mathrm{n}=25$
\end{tabular}


Saeed 2015 (Continued)

Control group (standard oxygen therapy): Venturi face mask; $\mathrm{n}=20$

Outcomes relevant to this review: ABG; treatment failure; successful weaning

Notes

Contact: Adel Saeed, Pulmonary Medicine, Ain Shams University, Abbasia, Cairo Egypt

Currently published only as an abstract. We are awaiting publication of the full report in order to assess eligibility, collect sufficient study characteristics, and include data in the review.

Schreiber 2017

\begin{tabular}{ll}
\hline Methods & RCT, cross-over design. Single-entre study \\
\hline Participants & $\begin{array}{l}\text { Total number of participants: } 20 \\
\text { Inclusion criteria: acute respiratory failure; spontaneously breathing patients } \\
\text { Exclusion criteria: none in abstract }\end{array}$ \\
\hline Interventions & Three 60 -minute trials with the following therapies in random order. \\
& $\begin{array}{l}\text { Intervention (HFNC): flow }=60 \text { L/min } \\
\text { Control 1: (NIV) } \\
\text { Control 2: (standard oxygen therapy) }\end{array}$ \\
\hline Outcomes & $\begin{array}{l}\text { All outcomes reported: lung ultrasound aeration score; diaphragm thickening fraction; diaphragm } \\
\text { excursion } \\
\text { Outcomes relevant to this review: none }\end{array}$ \\
\hline Notes & $\begin{array}{l}\text { Contact: Annia Fleur Schreiber, Respiratory Intensive Care Unit and Pulmonary Rehabilitation Unit, } \\
\text { Istituti Clinici Scientifici Maugeri IRCCS, Pavia, Italy } \\
\text { Currently published only as an abstract. We are awaiting publication of the full report in order to } \\
\text { assess eligibility, collect sufficient study characteristics, and include data in the review. }\end{array}$ \\
\hline
\end{tabular}

\section{Theerawit 2017}

\begin{tabular}{ll}
\hline Methods & RCT, parallel-group design. Single-centre study \\
\hline Participants & Number of participants: 88 \\
& Setting: medical ICU, Thailand \\
& Inclusion criteria: age $>18$; ready to wean from invasive MV; high risk of reintubation \\
& Exclusion criteria: none reported \\
\hline Interventions & Intervention (HFNC): $\mathrm{n}=43$ \\
& Control group (NIV, CPAP): $\mathrm{n}=45$ \\
Both administered for 48 hours
\end{tabular}

Outcomes

All outcomes measured: reintubation rate; respiratory failure; physiologic variables; mortality 
Outcomes relevant to this review: reintubation rate; respiratory failure; physiologic variables; mortality

\begin{tabular}{l} 
Notes \\
Contact: P. Theerawit, Ramathibodi Hospital, Bangkok, Thailand \\
$\begin{array}{l}\text { Currently published only as an abstract. We are awaiting publication of the full report in order to } \\
\text { assess eligibility, collect sufficient study characteristics, and include data in the review. }\end{array}$ \\
\hline
\end{tabular}

Tseng 2019

\begin{tabular}{|c|c|}
\hline Methods & RCT, parallel-group design. Single-centre study \\
\hline \multirow[t]{4}{*}{ Participants } & Total number of participants: 40 \\
\hline & Setting: stepdown unit for weaning, Taiwan \\
\hline & Inclusion criteria: prolonged MV (> 14 days); ready for weaning \\
\hline & $\begin{array}{l}\text { Exclusion criteria: neuromuscular disorder, central respiratory drive disorders, tracheostomy; do- } \\
\text { not-resuscitate order. }\end{array}$ \\
\hline \multirow[t]{2}{*}{ Interventions } & Intervention group: (HFNC) \\
\hline & Control group: (NIV) \\
\hline \multirow[t]{2}{*}{ Outcomes } & $\begin{array}{l}\text { All outcomes reported: reintubation rate; in-hospital mortality; length of NIV; physiologic variables } \\
\left(\mathrm{PaO}_{2} / \mathrm{FiO}_{2} \text { and } \mathrm{PaCO}_{2}\right)\end{array}$ \\
\hline & Outcomes relevant to this review: reintubation rate; in-hospital mortality; $\mathrm{PaO}_{2} / \mathrm{FiO}_{2}$ and $\mathrm{PaCO}_{2}$ \\
\hline \multirow[t]{2}{*}{ Notes } & Contact: Chi-Wei Tseng, kiwitseng724@gmail.com \\
\hline & $\begin{array}{l}\text { Currently published only as an abstract. We are awaiting publication of the full report in order to } \\
\text { assess eligibility, collect sufficient study characteristics, and include data in the review. }\end{array}$ \\
\hline
\end{tabular}

Yang 2019

\begin{tabular}{ll}
\hline Methods & RCT, parallel-group design \\
\hline Participants & Total number of participants: 74 \\
& Setting: China \\
& Inclusion criteria: acute exacerbation of COPD (Grade I/II) \\
& Exclusion criteria: none reported \\
\hline Interventions & Intervention group (HFNC): $\mathrm{n}=37 ;$ flow $=40 \mathrm{~L} / \mathrm{min}$ \\
& Control group (standard oxygen therapy): $\mathrm{n}=37 ;$ nasal cannula; flow $=3 \mathrm{~L} / \mathrm{min}$ \\
\hline Outcomes & $\begin{array}{l}\text { All outcomes measured: ultrasound } \mathrm{measure} \text { of diaphragm movement (shallow and deep breath- } \\
\text { ing); diaphragmatic fast breathing index; } \mathrm{PaO}_{2} ; \mathrm{PaCO}_{2}\end{array}$ \\
& Outcomes relevant to this review: $\mathrm{PaO}_{2} ; \mathrm{PaCO}_{2}$ \\
\hline
\end{tabular}


Yang 2019 (Continued)

Notes
Contact: Yang Shengqiang, Department of Intensive Medicine, Huxi Hospital (Shan County Central Hospital), Jining Medical College

We did not source the full text of this study which was published in Chinese; we have taken the current information from the English abstract. This study requires translation into English in order to be incorporated into the review. We will seek translation when completing the next review update.

Zhang 2018

\begin{tabular}{ll}
\hline Methods & RCT, parallel-group design. Single-centre study \\
\hline Participants & Total number of participants: 45 \\
& Setting: emergency department ("EICU"), China \\
& Inclusion criteria: COPD, invasive MV; ready for extubation \\
& Exclusion criteria: none in abstract \\
\hline Interventions & $\begin{array}{l}\text { Intervention group (HFNC): } \mathrm{n}=21 \\
\text { Control group (NIV): } \mathrm{n}=24\end{array}$ \\
\hline Outcomes 24 outcomes measured: incidence of aspiration; incidence of pressure ulcers; incidence of deliri- \\
um; oxygenation; PaCO 2 ; length of ICU stay; 28 -day mortality; reintubation rate \\
$\begin{array}{l}\text { Outcomes relevant to this review: oxygenation; PaCO } ; \text {; length of ICU stay; 28-day mortality; reintu- } \\
\text { bation rate }\end{array}$ \\
$\begin{array}{l}\text { Contact: Zhang Jingchen, Department of Emergency Medicine, The First Affiliated Hospital of Zhe- } \\
\text { jiang University School of Medicine, Zhejiang Provincial Poison Control Center } \\
\text { We did not source the full text of this study which was published in Chinese; we have taken the cur- } \\
\text { rent information from the English abstract. This study requires translation into English in order to } \\
\text { be incorporated into the review. We will seek translation when completing the next review update. }\end{array}$ \\
\hline
\end{tabular}

Zhao 2019

\begin{tabular}{ll}
\hline Methods & RCT, parallel-group design. Single-centre study \\
\hline Participants & Total number of participants: 60 \\
& Setting: ICU, China \\
& Inclusion criteria: elderly; advanced lung cancer; respiratory failure; admitted to hospital; PaO $2<$ \\
& $50 \mathrm{mmHg} ; \mathrm{PaCO}_{2}>60 \mathrm{mmHg}$ \\
& Exclusion criteria: type II respiratory failure \\
\hline Interventions & Intervention group (HFNC): $\mathrm{n}=30$ \\
& Control group (NIV): $\mathrm{n}=30$ \\
\hline
\end{tabular}

Outcomes

All outcomes measured: pulse; $\mathrm{SpO}_{2} ; \mathrm{PaO}_{2}$; damage to facial skin 
Zhao 2019 (Continued)

Outcomes relevant to this review: $\mathrm{SpO}_{2} ; \mathrm{PaO}_{2}$; damage to facial skin

Notes

Contact: Zhao Yue, Department of Respiratory and Critical Care Medicine, Jiangyin People's Hospital, Jiangyin, Jiangsu, 214400

This study was published in Chinese; we have taken the current information from the English abstract. This study requires translation into English in order to be incorporated into the review. We will seek translation when completing the next review update.

Zhu 2017

\begin{tabular}{|c|c|}
\hline Methods & $\mathrm{RCT}$, parallel-group design. Single-centre study \\
\hline \multirow[t]{4}{*}{ Participants } & Total number of randomized participants: 49 \\
\hline & Setting: China \\
\hline & Inclusion criteria: invasive MV; ready for extubation \\
\hline & Exclusion criteria: none reported \\
\hline \multirow[t]{2}{*}{ Interventions } & Intervention group (HFNC): $\mathrm{n}=25$ \\
\hline & Control group (NIV): $\mathrm{n}=24$ \\
\hline \multirow[t]{2}{*}{ Outcomes } & $\begin{array}{l}\text { All outcomes reported: ABG; sputum viscosity; nasal and facial pressure ulcers within } 7 \text { days; rein- } \\
\text { tubation; change of therapy }\end{array}$ \\
\hline & $\begin{array}{l}\text { Outcomes relevant to this review: ABG; nasal and facial pressure ulcers; reintubation; change of } \\
\text { therapy }\end{array}$ \\
\hline \multirow[t]{3}{*}{ Notes } & $\begin{array}{l}\text { Contact: Zhu Zhengfang, Department of Intensive Medicine, Tenth People's Hospital, Tongji Univer- } \\
\text { sity, Shanghai, } 200072\end{array}$ \\
\hline & Study dates: 1 January to 31 December 2016 \\
\hline & $\begin{array}{l}\text { We did not source the full text of this study which was published in Chinese; we have taken the cur- } \\
\text { rent information from the English abstract. This study requires translation into English in order to } \\
\text { be incorporated into the review. We will seek translation when completing the next review update. }\end{array}$ \\
\hline
\end{tabular}

ABG: arterial blood gas

AECOPD: acute exacerbation of COPD

ARF: acute respiratory failure

BiPAP: bilevel positive airway pressure

CAM-ICU: confusion assessment method for the ICU

COPD: chronic obstructive pulmonary disease

CPAP: continuous positive airway pressure

$\mathrm{FiO}_{2}$ : fraction of inspired oxygen

$\mathrm{HCO}_{3}$ : bicarbonate

HDU: high dependency unit

HFNC: high-flow nasal cannulae

ICU: intensive care unit

IPAP: inspiratory positive airway pressure

MV: mechanical ventilation

NIV: non-invasive ventilation

$\mathrm{PaCO}_{2}$ : partial pressure of carbon dioxide in arterial blood

$\mathrm{PaO}_{2}$ : partial pressure of oxygen in arterial blood

$\mathrm{pH}$ : potential of hydrogen

High-flow nasal cannulae for respiratory support in adult intensive care patients (Review) 
PEEP: positive end expiratory pressure

$\mathrm{RCT}$ : randomized controlled trial

RASS: Richmond agitation-sedation score

$\mathrm{SpO}_{2}$ : oxygen saturation

Characteristics of ongoing studies [ordered by study ID]

\section{ACTRN12617000694314}

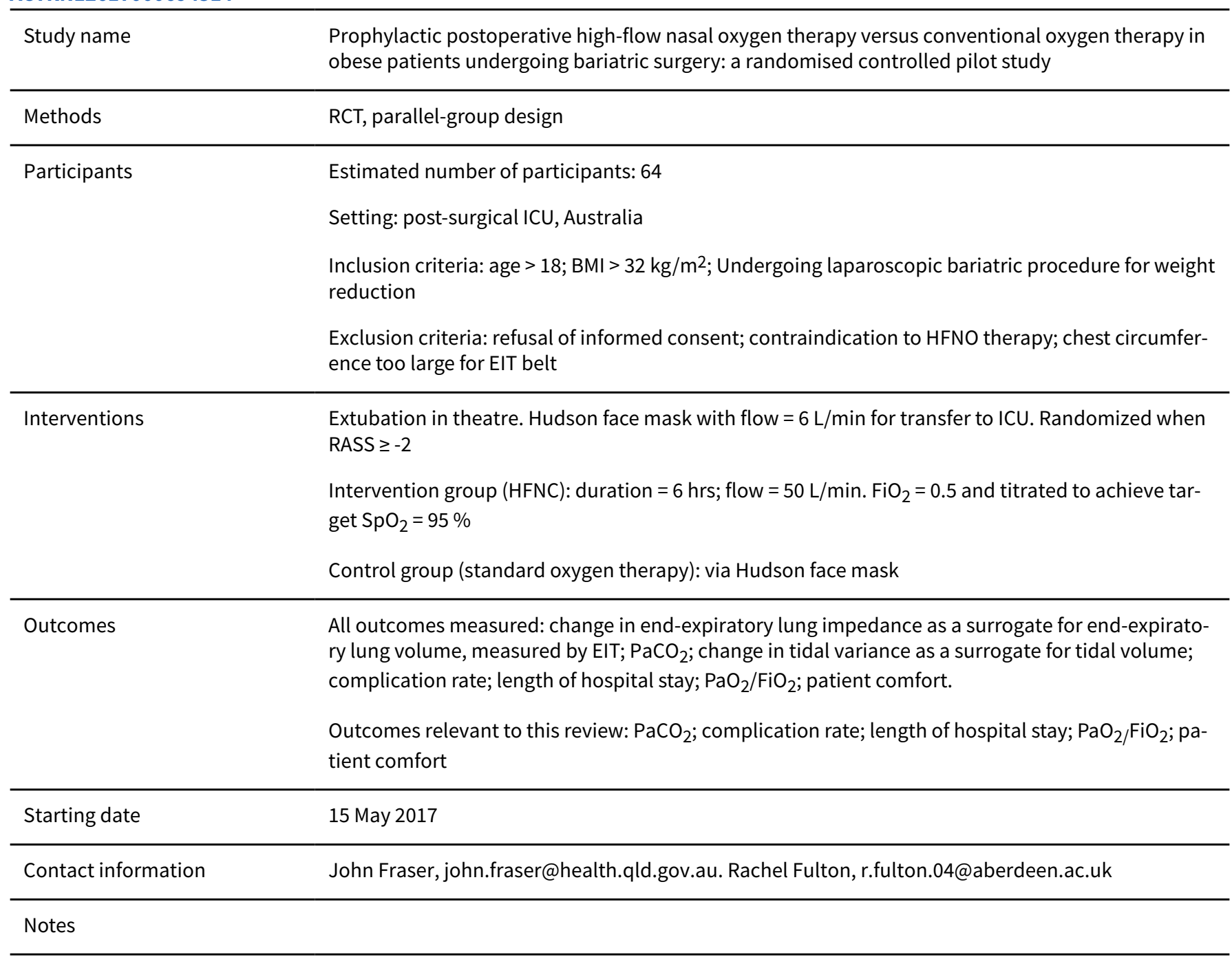

\section{ChiCTR1800014553}

Study name

Comparative study of nasal high-flow oxygen therapy and noninvasive positive pressure ventilation for moderate AECOPD: randomized open non-inferiority trial

\begin{tabular}{ll}
\hline Methods & RCT, parallel-group design \\
\hline Participants & Total number of participants: 86 \\
& Setting: China \\
& Inclusion criteria: AECOPD; blood gas analysis $\mathrm{pH} 7.25-7.35, \mathrm{PaCO}_{2}>50 \mathrm{mmHg}$
\end{tabular}


ChiCTR1800014553 (Continued)

Exclusion criteria: age < 18; no informed consent obtained; severe respiratory failure requiring tracheal intubation; NPPV contraindications; patients with short-term prognosis; other organ failure; tracheotomy

\begin{tabular}{ll}
\hline Interventions & Intervention group (HFNC) \\
Control group (NPPV)
\end{tabular}

\section{Outcomes}

All outcomes measured: arterial blood gas, respiratory rate, blood pressure, daily treatment time; parameter setting of NPPV and HFNC; change and time of respiratory support; intubation; time to intubation; dyspnoea score; comfort score; facial skin breakage; number of daily nursing interventions; respiratory and extrapulmonary complications; ICU length of stay; hospital length of stay; discharge outcome (death, improved)

Outcomes relevant to this review: arterial blood gas, respiratory rate, intubation; dyspnoea score; comfort score; respiratory and extrapulmonary complications; ICU length of stay; hospital length of stay; mortality

\begin{tabular}{ll}
\hline Starting date & 21 January 2018 \\
\hline Contact information & Dingyu Tan, 32494845@qq.com. Bingyu Ling \\
\hline Notes & \\
\hline
\end{tabular}

\section{ChiCTR1800017313}

\begin{tabular}{ll}
\hline Study name & Clinical application of high-flow nasal cannula therapy in patients with post-traumatic ARDS \\
\hline Methods & $\begin{array}{l}\text { Randomized controlled trial. Stratified randomization based on the oxygenation index } \mathrm{PaO}_{2} / \mathrm{FiO}_{2} \\
\text { divided to three patients groups, and randomly assigned to two treatment arms }\end{array}$
\end{tabular}

Participants

Estimated number of participants: 90

Setting: China

Inclusion criteria:

- Group 1: <24h of trauma; $\mathrm{PO}_{2} / \mathrm{FiO}_{2}=200-300 \mathrm{mmHg}$

- Group 2: <24h of trauma; $\mathrm{PO}_{2} / \mathrm{FiO}_{2}=200-300 \mathrm{mmHg}$

- Group 3: trauma; intubated; pass SBT

Exclusion criteria:

- Group 1: age < 18; informed consent not obtained; other organ failure; tracheotomy.

- Group 2: age < 18; informed consent not obtained; other organ failure; tracheotomy; severe ARDS requiring immediate intubation; NIV taboos.

- Group 3: age < 18; informed consent not obtained; NIV taboos; tracheotomy

\begin{tabular}{ll}
\hline Interventions & Intervention group (HFNC) \\
& Control group (NIV)
\end{tabular}

Outcomes

All outcomes measured: arterial blood gas; respiratory rate; blood pressure; change and time of respiratory support; whether invasive ventilation (endotracheal intubation) is required and time; dyspnoea score; comfort score; facial skin breakage; respiratory and extrapulmonary complications; length of stay in ICU; length of stay in hospital; discharge (death, healed/improved) 
ChiCTR1800017313 (Continued)

Outcomes relevant to this review: arterial blood gas; respiratory rate; whether invasive ventilation (endotracheal intubation) is required and time; dyspnoea score; comfort score; respiratory and extrapulmonary complications; length of stay in ICU; length of stay in hospital; discharge (death, healed/improved)

\begin{tabular}{ll}
\hline Starting date & 24 July 2017 \\
\hline Contact information & Li Yujie, 34504287@qq.com \\
\hline Notes & \\
\hline
\end{tabular}

\section{ChiCTR1800018530}

\section{Study name}

A multicenter randomized controlled trial for invasive-high-flow oxygen therapy and invasive-noninvasive sequential therapy for severe respiratory failure caused by COPD

\begin{tabular}{|c|c|}
\hline Methods & RCT, parallel-group design. Multicentre study \\
\hline \multirow[t]{4}{*}{ Participants } & Estimated number of participants: 168 \\
\hline & Setting: China \\
\hline & $\begin{array}{l}\text { Inclusion criteria: invasive respiratory failure due to type II respiratory failure; age 18-85; COPD; tak- } \\
\text { en care of themselves for the past } 1 \text { year; AECOPD due to bronchoalveolar infection; PIC window } \\
\text { appeared after invasive ventilation and anti-infection treatment }\end{array}$ \\
\hline & $\begin{array}{l}\text { Exclusion criteria: informed consent not available; NPPV contraindications; moribund; under pal- } \\
\text { liative care; severe heart, brain, liver and kidney failure; cough reflex extremely weak or sputum } \\
\text { weakness when PIC window appears; tracheostomy }\end{array}$ \\
\hline \multirow[t]{2}{*}{ Interventions } & Intervention group( HFNC) \\
\hline & Control group (NPPV) \\
\hline \multirow[t]{2}{*}{ Outcomes } & $\begin{array}{l}\text { All outcomes measured: treatment failure rate; respiratory parameters; dyspnoea score; comfort } \\
\text { score; skin ulceration score; } 28 \text {-day mortality }\end{array}$ \\
\hline & $\begin{array}{l}\text { Outcomes relevant to this review: treatment failure rate; respiratory parameters; dyspnoea score; } \\
\text { comfort score; 28-day mortality }\end{array}$ \\
\hline Starting date & 22 September 2018 \\
\hline Contact information & Dingyu Tan, 32494845@qq.com. Bingyu Ling, bingyuhope@163.com \\
\hline Notes & \\
\hline
\end{tabular}

\section{ChiCTR1900020826}

$\begin{array}{ll}\text { Study name } & \begin{array}{l}\text { A multicenter randomized controlled clinical trial for standardized respiratory support treatment } \\ \text { for acute severe virus pneumonia }\end{array}\end{array}$


ChiCTR1900020826 (Continued)

\section{Setting: China}

Inclusion criteria: age > 18 years; acute viral pneumonia

Exclusion criteria: indication for intubation and MV; received MV; hypercapnia; acute attack of bronchial asthma; acute exacerbation of COPD or other chronic lung diseases; cardiogenic pulmonary oedema; severe neutropenia; haemodynamic instability; GCS $<=12$; contraindications to NIV; facial or nasal deformities, nasal deformities; requires airway protection; tracheotomy; refuses endotracheal intubation; pregnancy

\begin{tabular}{ll}
\hline Interventions & $\begin{array}{l}\text { Intervention group (HFNC) } \\
\text { Control group (NIV) }\end{array}$ \\
\hline Outcomes & All outcomes reported: intubation rate; time to intubation \\
& Outcomes relevant to this review: intubation rate \\
\hline Starting date & 20 January 2019 \\
\hline Contact information & Liu Xiaoqing, lxq1118@126.com. Xi Yin, xiyin86@163.com \\
\hline Notes & \\
\hline
\end{tabular}

\section{ChiCTR1900021091}

Study name Improvement of ventilator weaning rate in patients with severe pneumonia by HFNC

\begin{tabular}{ll}
\hline Methods & RCT, parallel-group design \\
\hline Participants & Estimated number of participants: 160 \\
& Setting: ICU, China \\
& Inclusion criteria: severe pneumonia; extubated; survival time after disease > 2 weeks \\
& Exclusion criteria: failed SBT; poor sputum reflex \\
\hline Interventions & Intervention group (HFNC) \\
\hline Control group (standard oxygen therapy) \\
\hline All outcomes reported: length of ICU stay; success rate of extubation \\
\hline Outcomes relevant to this review: length of ICU stay; success rate of extubation \\
\hline Notes & 28 January 2019 \\
\hline
\end{tabular}




\section{ChiCTR1900022241}

Study name

Efficacy of high-flow nasal cannula oxygen therapy and noninvasive positive pressure ventilation for acute respiratory failure in patients with AIDS: a prospective, randomized, controlled trial

\section{$\mathrm{RCT}$, parallel-group design}

Estimated number of participants: 120

Setting: ICU or infectious disease department, China

Inclusion criteria: AIDS diagnosis; admitted to the ICU or to the infection disease department; AHRF; 18 to 70 years old; use of accessory muscles, paradoxical breathing; respiratory rate $>25$ breaths/min; course of AHRF after admission < 72 hours; agree to participate in this study and undergo tracheal intubation

Exclusion criteria: indication for emergency endotracheal intubation; anatomical factors precluding the use of a nasal cannula; hypercapnia indication NIV $\left(\mathrm{PaCO}_{2}=50 \mathrm{mmHg}\right)$; presence of pneumothorax or extensive pleural effusion; cardiorespiratory arrest; isolated cardiogenic pulmonary oedema indicating NIV; decreased level of consciousness (Glasgow < 13); persistent haemodynamic instability after requiring norepinephrine; other known immunosuppression; surgery with in the last 6 days; pregnant or breastfeeding

\begin{tabular}{ll}
\hline Interventions & Intervention (HFNC) \\
& Control group (NIV)
\end{tabular}

Outcomes

All outcomes measured: intubation rate within 14 days; 28-day mortality; 90-day mortality

Outcomes relevant to this review: intubation rate within 14 days; 90 -day mortality

\begin{tabular}{ll}
\hline Starting date & 31 March 2019 \\
\hline Contact information & Ang Li, dtyyicu@ccmu.edu.cn. Jingyuan Liu, dtyyicu@outlook.com \\
\hline Notes & \\
\hline
\end{tabular}

\section{ChiCTR1900023296}

\begin{tabular}{ll}
\hline Study name & $\begin{array}{l}\text { Sequential therapy effects of HFNC on patients with thoracic trauma combined with ARDS from } \\
\text { weaning: a prospective single-centered randomized controlled study }\end{array}$ \\
\hline Methods & RCT, parallel-group design. Single-centre study \\
\hline Participants & Estimated number of participants: 80 \\
& Setting: ICU, China \\
& $\begin{array}{l}\text { Inclusion criteria: age } 15-75 ; \text { within } 24 \text { hours after routine extubation; pulmonary contusion signif- } \\
\text { icantly improved; no haemopneumothorax; pleural effusion reduced; spontaneous expectoration } \\
\text { possible; temperature < 38.0 } 0^{\circ} \text {; tidal volume 3-5 mL/kg; haemodynamically stable; haemoglobin > }> \\
80 \text { g/L } \\
\text { Exclusion criteria: tracheotomy; craniocerebral trauma; disturbance of consciousness; other major } \\
\text { organ injuries; facial deformity }\end{array}$ \\
Intervention group (HFNC) \\
Control group (NIV): BiPAP
\end{tabular}


ChiCTR1900023296 (Continued)

Outcomes

All outcomes measured: mortality rate; oxygenation; $\mathrm{PaCO}_{2}$; reintubation rate; length of hospital stay

Outcomes relevant to this review: mortality rate; oxygenation; $\mathrm{PaCO}_{2}$; reintubation rate; length of hospital stay

\begin{tabular}{ll}
\hline Starting date & 21 May 2019 \\
\hline Contact information & Ma Li, mali1105@126.com \\
\hline
\end{tabular}

Notes

\section{ChicTR1900025974}

\begin{tabular}{ll}
\hline Study name & High-flow nasal cannula versus non-invasive positive pressure ventilation therapy after early extu- \\
& bation for patients with acute exacerbation of chronic obstructive pulmonary disease: a multicen- \\
ter randomized controlled trial
\end{tabular}

\begin{tabular}{|c|c|}
\hline Methods & $\mathrm{RCT}$, parallel-group design \\
\hline \multirow[t]{4}{*}{ Participants } & Estimated number of participants: 502 \\
\hline & Setting: ICU, China \\
\hline & $\begin{array}{l}\text { Inclusion criteria: } 48 \text { to } 85 \text { years old; COPD; hypercapnic respiratory failure due to bronchial-pul- } \\
\text { monary infection; treated with MV; intubated for at least } 48 \text { hours; meeting pulmonary infection } \\
\text { control window; self-care for the past year; informed consent }\end{array}$ \\
\hline & $\begin{array}{l}\text { Exclusion criteria: severe organ dysfunction; chronic neuromuscular disease; tracheotomy; upper } \\
\text { airway obstruction; facial injury or oral, oesophageal and gastric surgery within one month; home } \\
\text { NPPV; weak cough ability with copious secretions; terminal tumour; do-not-reintubate order }\end{array}$ \\
\hline \multirow[t]{2}{*}{ Interventions } & Intervention group (HFNC) \\
\hline & Control group (NPPV) \\
\hline \multirow[t]{2}{*}{ Outcomes } & $\begin{array}{l}\text { All outcomes measured: reintubation; weaning failure rate; HFNC failure rate; NPPV failure rate; } \\
\text { hospital mortality rate; comfort score; adverse reaction }\end{array}$ \\
\hline & $\begin{array}{l}\text { Outcomes relevant to this review: reintubation; weaning failure rate; HFNC failure rate; NPPV fail- } \\
\text { ure rate; hospital mortality rate; comfort score; adverse reaction }\end{array}$ \\
\hline Starting date & 16 September 2019 \\
\hline Contact information & Xie Lixin, xielx301@126.com. Han Xiaobo, hansir510@163.com \\
\hline Notes & \\
\hline
\end{tabular}

\section{ChiCTR-INR-17011850}

\begin{tabular}{ll}
\hline Study name & $\begin{array}{l}\text { Sequential oxygen therapy via high-flow nasal cannula following invasive ventilation in AECOPD in- } \\
\text { duced hypercapnic respiratory failure: a prospective randomized controlled study }\end{array}$ \\
\hline Methods & RCT, parallel-group design \\
\hline
\end{tabular}


ChiCTR-INR-17011850 (Continued)

\begin{tabular}{ll} 
Participants & Estimated number of participants: 36 \\
& $\begin{array}{l}\text { Setting: China } \\
\text { Inclusion criteria: acute exacerbation of COPD; respiratory failure; ready for extubation } \\
\text { Exclusion criteria: large amount of sputum or poor drainage; delirium; refusal of treatment; con- } \\
\text { traindication to NIV; haemoptysis; pneumothorax; pleural effusion; moribund; rhinitis }\end{array}$ \\
\hline Interventions & $\begin{array}{l}\text { Intervention group (HFNC) } \\
\text { Intervention group 2 (HFNC) } \\
\text { Control group (NIV) }\end{array}$ \\
\hline Outcomes & $\begin{array}{l}\text { All outcomes reported: ventilator-associated pneumonia; success rate of weaning; mortality rate; } \\
\text { vital signs; tolerance } \\
\text { Outcomes relevant to this review: ventilator-associated pneumonia; success rate of weaning; mor- } \\
\text { tality rate; vital signs; tolerance }\end{array}$ \\
\hline Starting date & 3 July 2017 \\
\hline Contact information & Guoqiang Jing, jingguoqiang2012@163.com. Xiaozhi Wang, jingguoqiang2012@163.com \\
\hline Notes & We are unsure how the two intervention groups differ. \\
\hline
\end{tabular}

\section{ChicTR-INR-17012720}

\begin{tabular}{|c|c|}
\hline Study name & $\begin{array}{l}\text { Application of high-flow nasal cannula oxygen therapy in patients with acute exacerbation of } \\
\text { chronic obstructive pulmonary disease }\end{array}$ \\
\hline Methods & $\mathrm{RCT}$, parallel-group design \\
\hline \multirow[t]{4}{*}{ Participants } & Estimated number of participants: 90 \\
\hline & Setting: ICU, China \\
\hline & $\begin{array}{l}\text { Inclusion criteria: } 18 \text { to } 80 \text { years old; AECOPD; respiratory failure treated with endotracheal intuba- } \\
\text { tion; admitted to ICU }\end{array}$ \\
\hline & $\begin{array}{l}\text { Exclusion criteria: other serious diseases, such as acute myocardial infarction, advanced tumours } \\
\text { etc.; serious malnutrition; severe pulmonary hypertension; facial trauma or facial deformities }\end{array}$ \\
\hline \multirow[t]{3}{*}{ Interventions } & Intervention group (HFNC) \\
\hline & Control group 1 (standard oxygen therapy): nasal catheter oxygen therapy \\
\hline & Control group 2 (NIV) \\
\hline \multirow[t]{2}{*}{ Outcomes } & $\begin{array}{l}\text { All outcomes measured: } 28 \text {-day reintubation rate; } 28 \text {-day mortality; length of ICU stay; length of } \\
\text { hospital stay }\end{array}$ \\
\hline & $\begin{array}{l}\text { Outcomes relevant to this review: } 28 \text {-day reintubation rate; } 28 \text {-day mortality; length of ICU stay; } \\
\text { length of hospital stay }\end{array}$ \\
\hline Starting date & 19 September 2017 \\
\hline Contact information & Yu Jiangquan, 15651057659@163.com \\
\hline Notes & \\
\hline
\end{tabular}


Cortegiani 2019

\section{Study name}

\section{Methods}

Participants
High-flow nasal therapy versus noninvasive ventilation in mild to moderate acute hypercapnic respiratory failure: a non-inferiority randomized trial
Setting: ICU, Italy
Inclusion criteria: COPD; AHRF; $7.25<\mathrm{pH}<7.35 ; \mathrm{PaCO}_{2} \leq 55 \mathrm{mmHg}$; age $>18$

Exclusion criteria: invasive MV in the last 60 days; use of NIV or HFNC prior to enrolment after onset of AHRF; NIV at home; unstable clinical condition; refusal of treatment; agitation or non-co-operation; failure of $>2$ organs; cardiac arrest; respiratory arrest requiring intubation; recent trauma or burns to the neck and face; pregnancy; refusal of consent; inclusion in other research protocols
Intervention group (HFNC): flow $=60 \mathrm{~L} / \mathrm{min}$. $\mathrm{FiO}_{2}$ titrated to achieve $\mathrm{SpO}_{2}=88-92 \%$

Control group(NIV): BiPAP; via face mask; IPAP set to achieve tidal volume $=6-8 \mathrm{~mL} / \mathrm{kg}$. PEEP $=3-5$ $\mathrm{mmH}_{2} \mathrm{O}$

All outcomes measured: $\mathrm{PaCO}_{2}$; dyspnoea; respiratory rate; discontinuation of interventions; overall discomfort; side effects; rate of treatment failure; rate of intubation

Outcomes relevant to this review: $\mathrm{PaCO}_{2}$; dyspnoea; respiratory rate; overall discomfort; side effects; rate of intubation

\begin{tabular}{ll}
\hline Starting date & 12 December 2017 \\
\hline Contact information & Andrea Cortegiani, cortegiania@gmail.com. Federico Longhini, federico.longhini@gmail.com. \\
\hline Notes & \\
\hline
\end{tabular}

\section{CTRI/2018/09/015717}

\begin{tabular}{|c|c|}
\hline Study name & High-flow oxygen through nasal cannula in acute hypoxemic respiratory failure \\
\hline Methods & $\mathrm{RCT}$, parallel-group design \\
\hline \multirow[t]{6}{*}{ Participants } & Estimated number of participants: 140 \\
\hline & Setting: ICU, India \\
\hline & Inclusion criteria: age $\geq 18$; respiratory rate $>25$ breaths $/ \mathrm{min} ; \mathrm{PaO}_{2} / \mathrm{FiO}_{2} \leq 300$ whilst breathing 10 \\
\hline & $\mathrm{L} / \mathrm{min} \mathrm{O}_{2}$ for 15 mins \\
\hline & Exclusion criteria: $\mathrm{PaCO}_{2}>45 \mathrm{mmHg}$, exacerbation of asthma or chronic respiratory failure; cardio- \\
\hline & $\begin{array}{l}\text { genic pulmonary oedema; history of chronic respiratory disease; haemodynamic instability; GCS } \\
\leq 12 \text {, contraindications to NIV, urgent need for intubation; palliative patients; tracheostomy; mori- } \\
\text { bund }<72 \text { hrs, respiratory failure due to potentially irreversible causes }\end{array}$ \\
\hline
\end{tabular}

\footnotetext{
Interventions
} Intervention group (HFNC): flow $=60 \mathrm{~L} / \mathrm{min}$, Fisher \& Paykel

Control group 1 (standard oxygen therapy): flow $\geq 10 \mathrm{~L} / \mathrm{min}$ non-rebreathe facemask 

ity rate; intubation rate with $\mathrm{PaO}_{2} / \mathrm{FiO}_{2}<200$; intubation rate in neutropenic participants; total duration of ICU stays; complication rate; dyspnoea

Outcomes relevant to this review: intubation rate within 28 days; ICU mortality rate; total duration of ICU stays; complication rate; dyspnoea

\begin{tabular}{ll}
\hline Starting date & 14 September 2018 \\
\hline Contact information & Dr Sheila Nainan Myatra, sheila150@hotmail.com \\
\hline
\end{tabular}

\section{Notes}

\section{ISRCTN16912075}

\begin{tabular}{ll}
\hline Study name & In adult patients with known or suspected COVID-19, does the use of continuous positive airway \\
pressure (CPAP) or high-flow nasal oxygen (HFNO), compared with standard care reduce mortality \\
or need for tracheal intubation?
\end{tabular}

\begin{tabular}{ll}
\hline Methods & RCT, parallel-group design. Single-centre study \\
\hline Participants & Estimated number of participants: 4002 \\
& Setting: ICU, UK \\
& Inclusion criteria: age $>=18 ;$ suspected or proven COVID-19; FiO $2>=40 \%$ with $\mathrm{SpO}_{2}<94 \% ;$ plan for \\
& intubation \\
& Exclusion criteria: planned intubation and MV within 1 hour; known pregnancy; contraindication \\
& to CPAP or HFNC; decision not to intubate due to ceiling of care; withdrawal of care anticipated; \\
& equipment for both CPAP and HFNC not available
\end{tabular}

\begin{tabular}{ll} 
Interventions & Intervention group (HFNC) \\
Control group 1 (NIV): CPAP \\
Control group 2 (standard oxygen therapy) \\
\hline
\end{tabular}

Outcomes

All outcomes reported: composite outcome comprising tracheal intubation or mortality within 30 days; intubation rate; time to intubation; time to death; mortality in critical care; hospital mortality; mortality at 30 days; ICU length of stay; hospital length of stay

Outcomes relevant to this review: intubation rate; hospital mortality; ICU length of stay; hospital length of stay

\begin{tabular}{ll}
\hline Starting date & 02 April 2020 \\
\hline Contact information & Keith Couper, k.couper@warwick.ac.uk. \\
\hline Notes & \\
\hline
\end{tabular}


Study name

Japanese, multicenter, randomized controlled trial of noninvasive positive pressure ventilation (NPPV) versus high-flow nasal cannula oxygen therapy (HFNC) for severe acute hypoxemic respiratory failure - Ja-NP-Hi trial

\begin{tabular}{ll}
\hline Methods & RCT, parallel-group design \\
\hline Participants & Estimated number of participants: 104 \\
& Setting: \\
& Inclusion criteria: acute respiratory failure, which occurred within 1 week of a known clinical insult \\
& or new or worsening respiratory symptoms; new infiltrates on chest radiography; $\mathrm{PaO}_{2} / \mathrm{FiO}_{2}<300$ \\
& at screening; $\mathrm{PaCO} \mathrm{C}_{2} \leq 45$ Torr at screening; age $>20 ;$ written informed consent \\
& Exclusion criteria: urgent need for endotracheal intubation; respiratory failure fully explained by \\
cardiac failure or fluid overload; exacerbation of asthma; pulmonary embolism; received NPPV or & HFNC for $>24$ hours at the time of the informed consent; chronic pulmonary disease; malignant \\
tumour affecting the efficacy endpoints; contraindications either to NPPV or $\mathrm{HFNC}$; major surgery \\
within 4 weeks; severe leukopenia; haemodynamic instability; need for vasopressors; $\mathrm{GCS} \leq 12$ \\
points; received NPPV or HFNC within the past 4 weeks prior to the informed consent; use of NPPV \\
or HFNC at home (more than 6 hours/day); tracheostomy; pregnancy; cognitive impairment or \\
mental disorder; participated in another study or will participate in another study; any other cases \\
who are regarded as inadequate for the study enrolment by the investigators
\end{tabular}

Interventions Intervention group (HFNC)

Control group (NPPV)

Outcomes

All outcomes measured: intubation rate; 28-day mortality; in-hospital mortality; ventilator-free days; oxygenation; arterial blood gas analysis; duration of respiratory support; length of hospital stay; adverse events; need for continuous sedation

Outcomes relevant to this review: intubation rate; 28-day mortality; oxygenation; arterial blood gas analysis; duration of respiratory support; length of hospital stay; adverse events

\begin{tabular}{ll}
\hline Starting date & 30 March 2019 \\
\hline Contact information & Kazuma Nagata, kazuma_n1101@yahoo.co.jp. Keisuke Tomii, ktomii@kcho.jp \\
\hline Notes & \\
\hline
\end{tabular}

\section{NCT01166256}

Study name
$\begin{aligned} & \text { Comparison between high-flow nasal cannula system and non-invasive ventilation in acute hypox- } \\ & \text { aemic respiratory failure }\end{aligned}$

\begin{tabular}{ll}
\hline Methods & RCT, parallel-group design \\
\hline Participants & Estimated number of participants: 74 \\
& Setting: Korea \\
& Inclusion criteria: age $\geq 18$ years, acute hypoxaemic respiratory failure \\
& Exclusion criteria: age < 18 years; $\mathrm{PaCO}_{2}>45 \mathrm{mmH}$ at admission; need for emergency intubation; \\
& cardiogenic shock or severe haemodynamic instability; lack of co-operation; altered mental status \\
& with decreased consciousness and/or evidence of inability to understand or lack of willingness to \\
& co-operate with procedures; tracheotomy or other upper airway disorders; severe ventricular ar-
\end{tabular}


NCT01166256 (Continued)

rhythmia or active myocardial ischaemia; active upper gastrointestinal bleeding; inability to clear respiratory secretions; > 1 severe organ dysfunction in addition to respiratory failure
Intervention group (HFNC): Optiflow, Fisher \& Paykel, Auckland, New Zealand; titrated to $\mathrm{SpO}_{2}>$ $92 \%$ or $\mathrm{PaO}_{2}>65 \mathrm{mmHg}$

Control group (NIV): BiPAP (Vision, Respironics Inc., Murrysville, PA); $\mathrm{S} / \mathrm{T}$ mode to achieve $\mathrm{SpO}_{2}>$ $92 \%$ or $\mathrm{PaO}_{2}>65 \mathrm{mmHg}$

\begin{tabular}{ll}
\hline Outcomes & $\begin{array}{l}\text { All outcomes reported: success rate of treatment; compliance with treatment; adverse event; hos- } \\
\text { pital length of stay; hospital mortality }\end{array}$ \\
& $\begin{array}{l}\text { Outcomes relevant to this review: success rate; adverse events; hospital length of stay; hospital } \\
\text { mortality }\end{array}$ \\
\hline Starting date & July 2010 \\
\hline Contact information & $\begin{array}{l}\text { Chae-Man Lim, MD, Department of Pulmonary and Critical Care Medicine, Asan Medical Center, } \\
\text { University of Ulsan College of Medicine, Seoul, Korea }\end{array}$ \\
\hline Notes &
\end{tabular}

\section{NCT01702779}

\begin{tabular}{ll}
\hline Study name & $\begin{array}{l}\text { Nasal humidified high-flow oxygen during weaning from mechanical ventilation: ultrasonography } \\
\text { study (HiFloLUS) }\end{array}$
\end{tabular}

\begin{tabular}{ll}
\hline Methods & RCT, parallel-group design \\
\hline Participants & Estimated number of participants: 80 \\
& Setting: ICU, France \\
& Inclusion criteria: adult patients ventilated $>48$ hours, stable respiratory and haemodynamic con- \\
& ditions for SBT, consent of participants, arterial line \\
& Exclusion criteria: COPD, laryngeal dyspnoea, tracheostomy, arrhythmia, no echogenicity, paraple- \\
& gia $>$ T8
\end{tabular}

Intervention group (HFNC): Optiflow

Control group (standard oxygen therapy)

$\begin{array}{ll}\text { Outcomes } & \text { All outcomes reported: variations in lung ultrasound score; lung ultrasound score; rate of post-ex- } \\ \text { tubation distress; EIT, epithelial and endothelial biomarkers }\end{array}$

Outcomes relevant to this review: rate of post-extubation distress

\begin{tabular}{ll}
\hline Starting date & August 2011 \\
\hline Contact information & Patrick Lacarin, University Hospital, Clermont-Ferrand, placarin@chu-clermonetferrand.fr \\
\hline Notes & ClinicalTrials.gov identifier: NCT01702779 \\
\hline
\end{tabular}


NCT02107183

Study name
Impact of nasal high-flow vs Venturi mask oxygen therapy on weaning outcome: a multicenter, randomized, controlled trial (RINO)

\begin{tabular}{|c|c|}
\hline Methods & RCT, parallel-group design. Multicentre study \\
\hline \multirow[t]{6}{*}{ Participants } & Estimated number of participants: 500 \\
\hline & Setting: ICU, Italy \\
\hline & $\begin{array}{l}\text { Inclusion criteria: age } \geq 18 \text { years, mechanical ventilation }>24 \text { hours, signed informed consent, suc- } \\
\text { cessful spontaneous breathing trial, } \mathrm{PaO}_{2} / \mathrm{FiO}_{2} \text { ratio } \leq 300\left(\text { or } \mathrm{SpO}_{2} / \mathrm{FiO}_{2} \text { ratio } \leq 300 \text { if } \mathrm{SpO}_{2}<98 \%\right)\end{array}$ \\
\hline & $\begin{array}{l}\text { within } 30 \text { minutes after extubation while breathing through a Venturi mask with a delivered } \mathrm{FiO}_{2} \text { of } \\
30 \%\end{array}$ \\
\hline & $\begin{array}{l}\text { Exclusion criteria: pregnancy, presence of tracheostomy, need for immediate post-extubation non- } \\
\text { invasive ventilation ( }>3 \text { consecutive failures of the spontaneous breathing trial and/or } \mathrm{PaCO}_{2}>45\end{array}$ \\
\hline & $\mathrm{mmHg}$ before spontaneous breathing trial, with respiratory rate $\geq 25 / \mathrm{min}$ ) \\
\hline \multirow[t]{3}{*}{ Interventions } & Intervention group (HFNC): Optiflow, Fisher \& Paykel Healthcare \\
\hline & Control group (standard oxygen therapy): Venturi mask \\
\hline & Both administered after extubation up to ICU discharge \\
\hline \multirow[t]{2}{*}{ Outcomes } & $\begin{array}{l}\text { All outcomes measures: reintubation; need for NIV; ICU length of stay; hospital length of stay; ICU } \\
\text { re-admission; ICU mortality; hospital mortality }\end{array}$ \\
\hline & $\begin{array}{l}\text { Outcomes relevant to this review: reintubation; need for NIV; ICU length of stay; hospital length of } \\
\text { stay; ICU mortality; hospital mortality }\end{array}$ \\
\hline Starting date & June 2014 \\
\hline Contact information & Salvatore Maurizio Maggiore, smmaggiore@rm.unicatt.it \\
\hline \multirow[t]{2}{*}{ Notes } & Completed in 2017 , however, we could not find a publication \\
\hline & ClinicalTrials.gov Identifier: NCT02107183 \\
\hline
\end{tabular}

\section{NCT02123940}

\begin{tabular}{ll} 
Study name & $\begin{array}{l}\text { Treatment strategy in patients with high-risk of post-extubation distress in ICU based on a lung ul- } \\
\text { trasound score versus standard strategy (WIN IN WEAN) }\end{array}$ \\
\hline Methods & RCT, parallel-group design. Multicentre study \\
\hline Participants & Estimated number of participants: 640 \\
& Setting: ICU, France \\
& $\begin{array}{l}\text { Inclusion criteria: adult patients ventilated }>48 \text { hours, stable respiratory and haemodynamic con- } \\
\text { ditions for SBT, consent of patients, arterial line }\end{array}$ \\
& $\begin{array}{l}\text { Exclusion criteria: severe COPD, laryngeal dyspnoea, tracheostomy, arrhythmia, no echogenicity, } \\
\text { paraplegia > T8 }\end{array}$ \\
\hline Interventions & Intervention group (HFNC) \\
\hline
\end{tabular}


NCT02123940 (Continued)

Outcomes

All outcomes measured: incidence of post-extubation distress, treatment failure (reintubation or curative non-invasive ventilation); number of ventilator-free days; length of stay in ICU; mortality in ICU

Outcomes relevant to this review: treatment failure; length of ICU stay; ICU mortality

\begin{tabular}{ll}
\hline Starting date & February 2014 \\
\hline Contact information & Patrick Lacarin, placarin@chu-clermonetferrand.fr \\
\hline Notes & ClinicalTrials.gov Identifier: NCT02123940 \\
\hline
\end{tabular}

\section{NCT02290548}

\section{Study name}

Effect of high-flow nasal oxygen vs standard oxygen therapy on extubation outcome with high risk of extubation failure in medical ICU patient

\begin{tabular}{|c|c|}
\hline Methods & $\mathrm{RCT}$, parallel-group design \\
\hline \multirow[t]{4}{*}{ Participants } & Estimated number of participants: 400 \\
\hline & Setting: ICU, Taiwan \\
\hline & $\begin{array}{l}\text { Inclusion criteria: MV > } 48 \text { hrs; age > } 65 \text { years; cardiac failure primary indication for MV; COPD; } \\
\text { bronchiectasis; old pulmonary tuberculosis with lung destruction; chronic renal failure; neuromus- } \\
\text { cular disease; } \mathrm{BMI}>30 \mathrm{~kg} / \mathrm{m}^{2} \text {; inability to manage respiratory secretions; ARDS }\end{array}$ \\
\hline & $\begin{array}{l}\text { Exclusion criteria: tracheostomy; recent facial trauma; active gastrointestinal bleeding; planned } \\
\text { NIV support post-extubation }\end{array}$ \\
\hline \multirow[t]{2}{*}{ Interventions } & Intervention group (HFNC): \\
\hline & Control group (standard oxygen therapy): nasal cannula or mask \\
\hline \multirow[t]{2}{*}{ Outcomes } & $\begin{array}{l}\text { All outcomes measured: reintubation rate; need for NIV; ICU readmission due to respiratory fail- } \\
\text { ure; ICU mortality; ICU length of stay; hospital mortality; hospital length of stay; hospital-acquired } \\
\text { pneumonia; desaturation to } \mathrm{SpO}_{2}<90 \% \text {; severe hypoxaemia } \mathrm{PaO}_{2} / \mathrm{FiO}_{2}<200 \text {; hypercapnia } \mathrm{PaCO}_{2} \\
>50 \text {; arterial } \mathrm{pH}<7.30 \text {; severe tachypnoea (respiratory rate }>40 \text { breaths } / \mathrm{min} \text { ) }\end{array}$ \\
\hline & $\begin{array}{l}\text { Outcomes relevant to this review: reintubation rate; need for NIV; ICU length of stay; hospital mor- } \\
\text { tality; hospital length of stay; hospital-acquired pneumonia }\end{array}$ \\
\hline Starting date & 14 November 2014 \\
\hline Contact information & KuoLi Kuo,Imn4093@gmail.com \\
\hline Notes & \\
\hline
\end{tabular}

\section{NCT02464696}

Study name $\quad \begin{aligned} & \text { Early non-invasive ventilation in patients with hypoxemic respiratory failure and hematological } \\ & \text { malignancies: a prospective randomized controlled trial }\end{aligned}$


NCT02464696 (Continued)

Participants
Estimated number of participants: 366

Setting: USA

Inclusion criteria: age $\geq 18 ; \mathrm{PaO}_{2} / \mathrm{FiO}_{2} \leq 300$ or $\mathrm{SpO}_{2} \leq 357$; diagnosed malignancy; chest radiograph or CT scan within $\leq 3$ months prior to enrolment to exclude primary or metastatic malignancy in the lungs or pleural spaces; probability of survival $\geq 6$ months

Exclusion criteria: do-not-resuscitate or do-not-intubate order; left heart failure primary cause of respiratory symptoms; active intrathoracic malignancy; accessory muscle use with breathing; shock; olgigouric acute renal failure; commenced NIV at time of screening; contraindications to NIV

Intervention group (HFNC): participants may receive NIV if indicated

Control group (NIV): alternating 2 hrs of NIV, $<2$ hrs NV with continuous NIV at night to achieve $\geq 8$ hrs/day; settings and $\mathrm{FiO}_{2}$ titrated to $\mathrm{SpO}_{2}>92 \%$; participant may receive HFNC; if contraindication to NIV develops, standard oxygen therapy can be used

All outcomes measured: intubation rate

Outcomes relevant to this review: intubation rate

\begin{tabular}{ll}
\hline Starting date & 8 June 2015 \\
\hline Contact information & Nisha Rathi, NRathi@mdanderson.org
\end{tabular}

\section{Notes}

\section{NCT02713737}

Study name

Impact of high-flow nasal cannula oxygen (HFNC) versus non-invasive ventilation associated with sleep quality on atrial fibrillation in hypoxemic patients after coronary surgery

\begin{tabular}{|c|c|}
\hline Methods & $\mathrm{RCT}$, parallel-group design \\
\hline \multirow[t]{4}{*}{ Participants } & Estimated number of participants: 80 \\
\hline & Setting: ICU, China \\
\hline & Inclusion criteria: hypoxemic respiratory failure. \\
\hline & Exclusion criteria: cardiac or respiratory arrest. \\
\hline \multirow[t]{2}{*}{ Interventions } & $\begin{array}{l}\text { Intervention group (HFNC): } \mathrm{FiO}_{2} \text { titrated to target } \mathrm{SpO}_{2}>92 \% \text {; Airvo, Fisher \& Paykel, Auckland, } \\
\text { New Zealand }\end{array}$ \\
\hline & $\begin{array}{l}\text { Control group (NIV): BiPAP; } \mathrm{FiO}_{2} \text { titrated to target } \mathrm{SpO}_{2}>92 \% \text {; TBird VELA ventilator, CareFusion, } \\
\text { USA; inspiratory pressure was raised every } 5 \text { mins until comfort was optimized }\end{array}$ \\
\hline \multirow[t]{2}{*}{ Outcomes } & $\begin{array}{l}\text { All outcomes measured: incidence of atrial fibrillation; } \mathrm{PaO}_{2} / \mathrm{FiO}_{2} \text {; lactate levels; intubation time; } \\
\text { transfusion requirement; inotropic usage; total sleep time; proportion of REM sleep; arousal index }\end{array}$ \\
\hline & Outcomes relevant to this review: $\mathrm{PaO}_{2} / \mathrm{FiO}_{2}$ \\
\hline Starting date & 21 March 20016 \\
\hline Contact information & You Zhang, 13598019682@126.com \\
\hline
\end{tabular}

High-flow nasal cannulae for respiratory support in adult intensive care patients (Review) 
NCT02713737 (Continued)

Notes

\section{NCT03014869}

Study name Comparison of high-flow nasal cannula and non-invasive positive ventilation (NPPV) in moderate chronic obstructive pulmonary disease exacerbation (AECOPD)

\begin{tabular}{ll}
\hline Methods & RCT, parallel-group design \\
\hline Participants & Estimated number of participants: 351 \\
& Setting: China \\
& Inclusion criteria: AECOPD; $7.35>\mathrm{pH} \geq 7.25, \mathrm{PaCO}_{2}>50 \mathrm{mmHg}$ \\
& $\begin{array}{l}\text { Exclusion criteria: contraindications for NPPV, such as thick sputum, cough weakness, haemody- } \\
\text { namic instability, etc.; need to be intubated immediately; refuse to engage in the study; severe or- } \\
\text { gan dysfunction }\end{array}$ \\
\hline Interventions & Intervention group (HFNC): flow $=25$ to 60 L/min. FiO 2 titrated to target SpO 2 of 90 to 95\%. \\
& Control group (NPPV) \\
\hline Outcomes & All outcomes measured: intubation demand within 90 days; intubation rate within 90 days. \\
\hline Outcomes relevant to this review: intubation rate within 90 days
\end{tabular}

\section{NCT03133520}

$\begin{array}{ll}\text { Study name } & \begin{array}{l}\text { Effectiveness of high-flow oxygen therapy in patients with hematologic malignancy acute hypox- } \\ \text { emic respiratory failure }\end{array}\end{array}$

\begin{tabular}{ll}
\hline Methods & RCT, parallel-group design \\
\hline Participants & Estimated number of participants: 50 \\
& Setting: Turkey \\
& Inclusion criteria: immunosuppression; haematological malignancy; $\mathrm{PaO}_{2} / \mathrm{FiO}_{2}<300 \mathrm{mmHg}$, or Pa- \\
& $\mathrm{CO}_{2} \leq 45 \mathrm{mmHg}$, or $\mathrm{SaO}_{2}<92 \%$; respiratory rate $>22$ breaths $/$ min \\
& $\begin{array}{l}\text { Exclusion criteria: refusal of study participation; pregnancy or breastfeeding; hypercapnia with for- } \\
\text { mal indication for NIV; treating physician decided NIV or invasive } \mathrm{MV} ; \text { haemodynamic instability; } \\
\text { need for vasopressors; confusion or disorientation }\end{array}$ \\
\hline Interventions & Intervention group (HFNC) \\
& Control group (standard oxygen therapy): nasal cannula or mask to achieve $\mathrm{SpO}_{2} \geq 95 \%$ \\
\hline
\end{tabular}


NCT03133520 (Continued)

Outcomes

All outcomes measured: 28-day mortality; patient comfort

Outcomes relevant to review: 28-day mortality; patient comfort

\begin{tabular}{ll}
\hline Starting date & 28 April 2017 \\
\hline Contact information & Kursat Gundogan, TC Erciyes University \\
\hline Notes & \\
\hline
\end{tabular}

\section{NCT03171935}

Study name

Wean early with high-flow nasal cannula oxygenation versus noninvasive positive pressure ventilation in patients with acute hypoxemic respiratory failure: a multicenter, randomized, controlled trial (the WHEN study)

\begin{tabular}{|c|c|}
\hline Methods & RCT, parallel-group design. Multicentre study \\
\hline \multirow[t]{5}{*}{ Participants } & Estimated number of participants: 270 \\
\hline & Setting: ICU, China \\
\hline & Inclusion criteria: endotracheal intubation; $\mathrm{PaO}_{2}<60 \mathrm{mmHg}$ (Venturi mask, $\mathrm{FiO}_{2}=0.5$ ) and $\mathrm{PaCO}_{2} \leq$ \\
\hline & $45 \mathrm{mmHg}$; meeting criteria for weaning; SBT failure \\
\hline & $\begin{array}{l}\text { Exclusion criteria: age }<18 ; \mathrm{MV}<48 \mathrm{hrs} \text {; tracheotomy; percentage of cuff leak in tidal volume }< \\
15.5 \% \text {; unable to spontaneously clear secretions from their airway; recent oral, nasal, facial or cra- } \\
\text { nial trauma or surgery; recent gastrointestinal bleeding; severe abdominal distention; lack of co- } \\
\text { operation; COPD, asthma, interstitial lung disease, neuromuscular disease }\end{array}$ \\
\hline \multirow[t]{4}{*}{ Interventions } & Intervention group (HFNC): flow $=50 \mathrm{~L} / \mathrm{min} ; \mathrm{FiO}_{2}=1.0$ then titrated to $\mathrm{SpO}_{2} \geq 92 \%$ \\
\hline & Control group 1 (NIV): Respironics V60, Philips; $\mathrm{FiO}_{2}$ titrated to $\mathrm{SpO}_{2} \geq 96 \%$; PEEP initially $4 \mathrm{~cm} \mathrm{H}_{2} \mathrm{O}$ \\
\hline & $\begin{array}{l}\text { increasing to a maximum of } 12 \mathrm{~cm} \mathrm{H}_{2} \mathrm{O} \text {; IPAP initially } 8 \mathrm{~cm} \mathrm{H} 2 \mathrm{O} \text { increasing to obtain a tidal volume } \\
\text { of } 6 \text { to } 8 \mathrm{~mL} / \mathrm{kg}\end{array}$ \\
\hline & Control group 2 (standard oxygen therapy): Venturi mask \\
\hline \multirow[t]{2}{*}{ Outcomes } & All outcomes reported: duration of invasive MV \\
\hline & Outcomes relevant to this review: none \\
\hline Starting date & 31 May 2017 \\
\hline Contact information & Zujin Luo, xmjg2002@163.com \\
\hline Notes & \\
\hline
\end{tabular}

\section{NCT03229460}

Study name

High-flow nasal oxygen therapy in perioperative period of the adult with hypercapnic and hypoxemic respiratory failure

Methods

$\mathrm{RCT}$, parallel-group design 
NCT03229460 (Continued)

Participants
Estimated number of participants: 150

Setting: China

Inclusion criteria: AHRF; severe dyspnoea at rest with respiratory rate $>25$ breaths $/ \mathrm{min} ; \mathrm{PaO}_{2} / \mathrm{FiO}_{2}$

$<300 ; \mathrm{PaCO}_{2}<45 \mathrm{mmHg}$

Exclusion criteria: age < 18 years; contraindications to NIV; chronic respiratory disease; cardiac pulmonary oedema; predefined intubation; haemodynamic instability or need for vasopressors; GCS $\leq$ 12; profound leukopenia

Interventions Intervention group (HFNC): flow $=30$ to $60 \mathrm{~L} / \mathrm{min} ; \mathrm{FiO}_{2}$ adjusted to target $\mathrm{SpO}_{2}>92 \%$

Control group 1 (standard oxygen therapy): flow $\geq 10 \mathrm{~L} / \mathrm{min}$

Control group 2 (NIV): face mask; $\mathrm{FiO}_{2}$ and/or PEEP adjusted to target $\mathrm{SpO}_{2}>92 \%$

Outcomes relevant to this review: intubation rate

\begin{tabular}{ll}
\hline Starting date & 25 July 2017 \\
\hline Contact information & Bin He, hebinicu@139.com. Dongjuan Tang, 317582862@qq.com \\
\hline
\end{tabular}

Notes

\section{NCT03246893}

$\begin{array}{ll}\text { Study name } & \begin{array}{l}\text { Efficacy of high-flow oxygen nasal cannula versus non-invasive positive pressure ventilation after } \\ \text { extubation in sepsis patients }\end{array}\end{array}$

\begin{tabular}{|c|c|}
\hline Methods & RCT, parallel-group design \\
\hline \multirow[t]{4}{*}{ Participants } & Estimated number of participants: 210 \\
\hline & Setting: ICU, Thailand \\
\hline & $\begin{array}{l}\text { Inclusion criteria: diagnosis of sepsis; depended on MV for }>48 \mathrm{hrs} \text {; plan for extubation due to suc- } \\
\text { cessful weaning }\end{array}$ \\
\hline & $\begin{array}{l}\text { Exclusion criteria: tracheostomy; recent upper abdominal surgery; facial injury; participant or rela- } \\
\text { tive did not agree to participate in the trial; physician preference for NIV or HFNC }\end{array}$ \\
\hline \multirow[t]{2}{*}{ Interventions } & Intervention group (HFNC): flow $=30 \mathrm{~L} / \mathrm{min} . \mathrm{FiO}_{2}=40$ to $60 \%$ \\
\hline & $\begin{array}{l}\text { Control group (NIV): face mask; IPAP }=6 \text { to } 8 \mathrm{~cm} \mathrm{H}_{2} \mathrm{O} \text {. PEEP }=3 \text { to } 5 \mathrm{~cm} \mathrm{H}_{2} \mathrm{O} . \mathrm{FiO}_{2}=30 \text { to } 60 \% \text {. Respi- } \\
\text { ratory rate }=12 \text { to } 16 \text { breaths } / \mathrm{min}\end{array}$ \\
\hline
\end{tabular}

\section{Outcomes}

All outcomes measured: reintubation; patient discomfort; change to another device within 72 hours; 28-day mortality; hospital mortality rate

Outcomes relevant to this review: reintubation; patient discomfort; 28-day mortality; hospital mortality rate 
NCT03246893 (Continued)

\section{NCT03282552}

\begin{tabular}{|c|c|}
\hline Study name & High-flow oxygen therapy versus conventional oxygen therapy in cardiac surgery patients \\
\hline Methods & RCT, parallel-group design \\
\hline \multirow[t]{4}{*}{ Participants } & Estimated number of participants: 99 \\
\hline & Setting: cardiac ICU, Greece \\
\hline & $\begin{array}{l}\text { Inclusion criteria: age }>18 \text { years; post-elective or urgent cardiac surgery; successful SBT with T- } \\
\text { piece and } \mathrm{FiO}_{2}=60 \% ; \mathrm{PaO}_{2} / \mathrm{FiO}_{2}<200 \text {; haemodynamically stable }(160>\mathrm{SAP}>90 \mathrm{mmHg})\end{array}$ \\
\hline & $\begin{array}{l}\text { Exclusion criteria: OSA supported by CPAP; COPD; tracheostomy; do-not-resuscitate order; GCS < } \\
\text { 13; insufficient knowledge of Greek language; visual or hearing impairment }\end{array}$ \\
\hline \multirow[t]{3}{*}{ Interventions } & Intervention group 1 (HFNC): $\mathrm{FiO}_{2}=60 \%$; flow $=60 \mathrm{~L} / \mathrm{min}$ \\
\hline & Intervention group 2 (HFNC): $\mathrm{FiO}_{2}=60 \%$; flow $=40 \mathrm{~L} / \mathrm{min}$ \\
\hline & $\begin{array}{l}\text { Control group (standard oxygen therapy): oxygen treatment according to the standard practice of } \\
\text { our cardiac ICU department, i.e. Venturi mask with } \mathrm{FiO}_{2}=60 \% \text { and flow }=15 \mathrm{~L} / \mathrm{min}\end{array}$ \\
\hline \multirow[t]{2}{*}{ Outcomes } & $\begin{array}{l}\text { All outcomes reported: successful weaning; maintaining respiratory rate of } 12-20 \text { breaths } / \mathrm{min} \text {; } \\
\mathrm{PaO}_{2} / \mathrm{FiO}_{2} \text { at } 48 \text { h or ICU discharge; maintaining } \mathrm{SpO}_{2} ; \text { use of accessory respiratory muscles; com- } \\
\text { fort }\end{array}$ \\
\hline & $\begin{array}{l}\text { Outcomes relevant to this review: successful weaning; respiratory rate; } \mathrm{PaO}_{2} / \mathrm{FiO}_{2} \text { at } 48 \mathrm{~h} \text { or ICU } \\
\text { discharge; } \mathrm{SpO}_{2} \text {; comfort }\end{array}$ \\
\hline Starting date & 14 September 2017 \\
\hline Contact information & Spiros Zakynthinos, National and Kapodistrian University of Athens \\
\hline Notes & Study completed 17 October 2019 . We could not find a full text. \\
\hline
\end{tabular}

\section{NCT03361683}

Methods RCT, parallel-group design

Participants

Estimated number of participants: 127

Setting: ICU, Mexico

Inclusion criteria: primary AHRF; invasive MV for $\geq 48$ hrs; successful SBT; age $\geq 18$ 
NCT03361683 (Continued)

Exclusion criteria: immediate indication for invasive MV or NIV; self-extubation; $\geq 1$ failed SBT; chronic respiratory failure; neuromuscular diseases; tracheostomy; nasal cavity pathology; facial surgery; failure to authorize the informed consent

Interventions

Intervention group (HFNC): flow $=40 \mathrm{~L} / \mathrm{min}$

Control group (standard oxygen therapy): Venturi mask; flow $=15 \mathrm{~L} / \mathrm{min}$
All outcomes measured: pulse, respiratory rate; median arterial pressure; $\mathrm{FiO}_{2} ; \mathrm{SpO}_{2}$; dyspnoea; comfort (all measured at 0, 1, 2, 3, 6, 12, 24 and 48 hours); ABG at 60 minutes and 24 hrs post-extubation; extubation failure (need for invasive MV within 2 days); extra supplementary oxygen through any device; time to $\mathrm{SpO}_{2}>94 \%$ on room air

Outcomes relevant to this review: extubation failure; respiratory rate; $\mathrm{SpO}_{2}$; comfort; dyspnoea; $A B G$

\begin{tabular}{ll}
\hline Starting date & 5 December 2017 \\
\hline Contact information & $\begin{array}{l}\text { Jose de Jesus Rodriguez-Andoney, National Institute of Medical Science and Nutrition Salvador Zu- } \\
\text { birán }\end{array}$ \\
\hline
\end{tabular}

\section{NCT03430258}

\begin{tabular}{|c|c|}
\hline Study name & High-flow nasal cannula oxygen therapy with the chest trauma patients \\
\hline Methods & RCT, parallel-group design. Single-centre study \\
\hline \multirow[t]{4}{*}{ Participants } & Estimated number of participants: 90 \\
\hline & Setting: emergency ICU; China \\
\hline & $\begin{array}{l}\text { Inclusion criteria: moderate to severe blunt thoracic injury (abbreviated injury scale chest score } \geq \\
\text { 3); admitted to the ICU }\end{array}$ \\
\hline & $\begin{array}{l}\text { Exclusion criteria: intubated or used MV within } 2 \mathrm{hrs;} \text { emergency surgery within } 2 \mathrm{hrs;} \text { unable to as- } \\
\text { sess using transthoracic ultrasound (severe subcutaneous emphysema or pneumothorax; GCS }<8\end{array}$ \\
\hline \multirow[t]{2}{*}{ Interventions } & Intervention group (HFNC): delivered by Optiflow nasal cannula using AIRVO 2 humidifier \\
\hline & Control group (standard oxygen therapy): nasal cannula or non-rebreathe mask \\
\hline \multirow[t]{2}{*}{ Outcomes } & All outcomes reported: intubation rate; lung aeration; ICU length of stay \\
\hline & Outcomes relevant to this review: intubation rate; ICU length of stay \\
\hline Starting date & 12 February 2018 \\
\hline Contact information & No contact details available \\
\hline Notes & \\
\hline
\end{tabular}


NCT03488628

Study name

High-flow nasal oxygen therapy for exacerbation of chronic pulmonary obstructive disease: a randomized, open-label, single-center, pilot study

\begin{tabular}{|c|c|}
\hline Methods & RCT, parallel-group design. Single-centre study \\
\hline \multirow[t]{4}{*}{ Participants } & Estimated number of participants: 44 \\
\hline & Setting: ICU, France \\
\hline & $\begin{array}{l}\text { Inclusion criteria: age > 18; COPD; respiratory rate raised or use of accessory respiratory muscles; } \\
\text { moderate exacerbation of COPD; admitted to ICU }<24 \text { hours prior to randomization. }\end{array}$ \\
\hline & $\begin{array}{l}\text { Exclusion criteria: pregnancy; sleep apnoea; NIV treatment at home; not affiliated to French social } \\
\text { security; contraindication to either NIV or HFNC; previous inclusion in the study }\end{array}$ \\
\hline \multirow[t]{2}{*}{ Interventions } & Intervention group (HFNC): delivered continuously for $24 \mathrm{hrs}$; target $\mathrm{SpO}_{2}=88$ to $92 \%$ \\
\hline & $\begin{array}{l}\text { Control group (NIV): alternated with conventional nasal oxygen therapy for } 24 \mathrm{hrs;} \text { target } \mathrm{SpO}_{2}=88 \\
\text { to } 92 \%\end{array}$ \\
\hline \multirow[t]{2}{*}{ Outcomes } & $\begin{array}{l}\text { All outcomes measured: arterial pH at } 24 \text { hours; } \mathrm{PaO}_{2} / \mathrm{FiO}_{2} \text { at } 24 \text { hours; incidence of tracheal intu- } \\
\text { bation; time course of arterial pH; time course of } \mathrm{SpO}_{2} ; \mathrm{PaCO}_{2} \text {; dyspnoea; perceived nursing work- } \\
\text { load; proportion of HFNC group who need NIV within } 24 \text { hours; severe adverse events }\end{array}$ \\
\hline & $\begin{array}{l}\text { Outcomes relevant to this review: } \mathrm{PaO}_{2} / \mathrm{FiO}_{2} \text { at } 24 \text { hours; incidence of tracheal intubation; time } \\
\text { course of } \mathrm{SpO}_{2} ; \mathrm{PaCO}_{2} ; \text { dyspnoea; proportion of } \mathrm{HFNC} \text { group who need NIV within } 24 \text { hours; severe } \\
\text { adverse events }\end{array}$ \\
\hline Starting date & 5 April 2018 \\
\hline Contact information & $\begin{array}{l}\text { Aurélie Despujols, aurelie.despujols@chr-orleans.fr. Thierry Boulain, thierry.boulain@chr-orlean- } \\
\text { s.fr. }\end{array}$ \\
\hline Notes & \\
\hline
\end{tabular}

\section{NCT03515031}

\begin{tabular}{ll}
\hline Study name & High-flow nasal cannulae vs venturi mask in respiratory failure due to pneumonia \\
\hline Methods & RCT, parallel-group design \\
\hline Participants & Estimated number of participants: 150 \\
& Setting: Italy \\
& Inclusion criteria: male or female; any ethnic group; age $\geq 18$ years; respiratory rate at rest $\geq 30$ \\
& breaths/minute or presence of respiratory distress; PaO 2 / FiO $2 \leq 250$ during oxygen therapy; diag- \\
& nosis of pneumonia as the unique cause of acute respiratory failure; informed consent obtained \\
& from the patient or the closest relative \\
& $\begin{array}{l}\text { Exclusion criteria: other diagnoses (instead of pneumonia) as a cause of acute respiratory fail- } \\
\text { ure; unstable angina and acute myocardial infarction in place; acute respiratory acidosis; systolic } \\
\text { blood pressure < } 90 \text { mmHg unresponsive to fluids or with amines; severe arrhythmias; epileptic } \\
\text { seizures; impaired swallowing; craniofacial trauma or burns; unco-operative patient; presence of } \\
\text { open wound (skull, chest, abdomen); respiratory arrest or need for intubation; ongoing pregnancy } \\
\text { or suspected }\end{array}$ \\
\hline
\end{tabular}


NCT03515031 (Continued)

Interventions
Intervention group ( $\mathrm{HFNC}$ ): flow $\geq 60 \mathrm{~L} / \mathrm{min}, \mathrm{FiO}_{2}$ titrated to target $\mathrm{SpO}_{2} \geq 92 \%$

Control group (standard oxygen therapy): Venturi mask

$\mathrm{FiO}_{2}$ titrated to target $\mathrm{SpO}_{2} \geq 92 \%$ in both groups

\begin{tabular}{ll}
\hline Outcomes & $\begin{array}{l}\text { All outcomes reported: endotracheal intubation; 30-day mortality; improvement of respiratory ex- } \\
\text { changes compared to baseline; arterial blood gas; adverse events; length of hospital stay } \\
\text { Outcomes relevant to this review: endotracheal intubation; 30-day mortality; arterial blood gas; } \\
\text { adverse events; length of hospital stay }\end{array}$ \\
\hline Starting date & 3 May 2018 \\
\hline Contact information & Roberto Cosentini, Papa Giovanni XXIII Hospital \\
\hline Notes &
\end{tabular}

\section{NCT03607357}

$\begin{array}{ll}\text { Study name } & \text { The effect of post-extubation high-flow nasal oxygen in patients with acute left heart failure: a clini- } \\ \text { cal multi-center study }\end{array}$
cal multi-center study

\begin{tabular}{|c|c|}
\hline Methods & RCT, parallel-group design. Multicentre study \\
\hline \multirow[t]{4}{*}{ Participants } & Estimated number of participants: 120 \\
\hline & Setting: ICU, China \\
\hline & $\begin{array}{l}\text { Inclusion criteria: clinical diagnosis of acute left heart failure; invasive MV > } 24 \text { hours; passes spon- } \\
\text { taneous breathing trials; next of kin agrees to sign the informed consent }\end{array}$ \\
\hline & $\begin{array}{l}\text { Exclusion criteria: COPD; disturbance of consciousness; bulbar paralysis, dysphagia; facial deformi- } \\
\text { ty; terminal tumour; neuromuscular disease }\end{array}$ \\
\hline \multirow[t]{3}{*}{ Interventions } & $\begin{array}{l}\text { Intervention group }(\mathrm{HFNC}) \text { : flow }=30 \mathrm{~L} / \text { min increasing in } 5 \mathrm{~L} / \text { min increments to tolerance; } \mathrm{FiO}_{2} \\
\text { titrated to } \mathrm{SpO}_{2}>95 \%\end{array}$ \\
\hline & Control group (NIV): BiPAP; IPAP = 6 to $8 \mathrm{~cm} \mathrm{H}_{2} \mathrm{O} ;$ PEEP $=4 \mathrm{~cm} \mathrm{H}_{2} \mathrm{O}$ \\
\hline & $\mathrm{FiO}_{2}$ titrated to $\mathrm{SpO}_{2}>95 \%$ for both groups \\
\hline \multirow[t]{2}{*}{ Outcomes } & $\begin{array}{l}\text { All outcomes measured: reintubation within } 48 \text { hours; } 28 \text {-day mortality; hospitalisation time; } \\
\text { length of ICU stay; blood gas analysis }\end{array}$ \\
\hline & $\begin{array}{l}\text { Outcomes relevant to this review: reintubation within } 48 \text { hours; 28-day mortality; hospitalisation } \\
\text { time; length of ICU stay; blood gas analysis }\end{array}$ \\
\hline Starting date & 31 July 2018 \\
\hline Contact information & Zhenglong Ye, zlyenj@126.com. Yuan Ding, 446490001@qq.com \\
\hline Notes & \\
\hline
\end{tabular}


NCT03632577

$\begin{array}{ll}\text { Study name } & \text { High-flow oxygen (HFO) versus non-invasive ventilation (NIV) associated to automated flow oxygen } \\ & \text { titration (AFOT) after extubation in patient with respiratory risk: non-inferiority prospective com- } \\ \text { parative study }\end{array}$

\begin{tabular}{ll}
\hline Methods & RCT, parallel-group design \\
\hline Participants & Estimated number of participants: 60 \\
& Setting: ICU, France \\
& $\begin{array}{l}\text { Inclusion criteria: respiratory disease due to suspected or proved COPD, asthma, bronchiectasis, } \\
\text { Cystic fibrosis, interstitial pneumonia, obstructive insufficient respiratory, restrictive insufficient } \\
\text { respiratory; extubation scheduled; informed consent given; affiliated to social insurance }\end{array}$ \\
& $\begin{array}{l}\text { Exclusion criteria: pregnancy; moribund; previous home NIV (not CPAP); tracheostomy; participant } \\
\text { under trusteeship, guardianship or safeguard of justice }\end{array}$
\end{tabular}

Interventions Intervention group (HFNO)

Control group (NIV): BiPAP

Outcomes

All outcomes measured: comfort; dyspnoea; treatment failure; $\mathrm{PaO}_{2} ; \mathrm{PaCO}_{2}$; length of hospital stay; length of ICU stay; 1-month mortality; 3-month mortality; ICU mortality; hospital mortality; use of another device in 72 hours; respiratory congestion; intubation rate at 48 hours; intubation rate at 72 hours; $\mathrm{SpO}_{2}$ stability

Outcomes relevant to this review: discomfort; dyspnoea; treatment failure; $\mathrm{PaO}_{2} ; \mathrm{PaCO}_{2} ;$ length of hospital stay; length of ICU stay; 3-month mortality

\begin{tabular}{ll}
\hline Starting date & 15 August 2018 \\
\hline Contact information & Elise Noel-Savina, noel-savina.e@chu-toulouse.fr \\
\hline Notes & \\
\hline
\end{tabular}

\section{NCT03643939}

$\begin{array}{ll}\text { Study name } & \begin{array}{l}\text { High-flow nasal oxygen cannula compared to non-invasive ventilation in adult patients with acute } \\ \text { respiratory failure }\end{array}\end{array}$

\begin{tabular}{|c|c|}
\hline Methods & RCT, parallel-group design \\
\hline Participants & $\begin{array}{l}\text { Estimated number of participants: } 1200 \\
\text { Setting: ICU or ED, Brazil } \\
\text { Inclusion criteria: age } \geq 18 \text {; admitted to ICU or ED; acute onset respiratory distress; } \\
\text { - } \quad \text { Non-immunocompromised AHRF: hypoxaemia evidenced by } \mathrm{SpO}_{2}<90 \% \text { or } \mathrm{PaO}_{2}<60 \mathrm{mmHg} \\
\text { in room air; use of accessory muscles, paradoxical breathing, and/or thoracoabdominal asyn- } \\
\text { chrony; respiratory rate }>25 \text { breaths/min } \\
\text { - Immunocompromised AHRF: as non-immunocompromised } \mathrm{AHRF} \text {; immunosuppression } \\
\text { - } \mathrm{AECOPD:} \mathrm{diagnosis} \mathrm{or} \mathrm{suspicion} \mathrm{of} \mathrm{COPD;} \mathrm{respiratory} \mathrm{rate}>25 \text { breaths/min; pH }<7.35 \text {; } \mathrm{PaCO}_{2}> \\
45 \text { mm Hg } \\
\text { - Cardiogenic acute pulmonary oedema: diagnosis of cardiogenic acute pulmonary oedema; respi- } \\
\text { ratory rate }>25 \text { breaths/min; } \mathrm{SpO}_{2}<95 \%\end{array}$ \\
\hline
\end{tabular}


NCT03643939 (Continued)

Exclusion criteria: indication for emergency intubation; psychomotor agitation requiring sedation; persistent haemodynamic instability; contraindications to NIV; pneumothorax or extensive pleural effusion; severe arrhythmia; thoracic trauma main cause of AHRF; asthma attack; pregnancy; cardiogenic shock; acute coronary syndrome; AHRF post-extubation; post-surgical AHRF; hypercapnic AHRF due to neuromuscular disease or chest deformity; exclusive palliative care; do-not-intubate order

Interventions Intervention group (HFNC): Airvo2, Fisher \& Paykel, Auckland, New Zealand

Control group (NIV): oronasal or full face mask
All outcomes reported: intubation rate; 90-day mortality; ICU-free days; MV-free days; hospital length of stay; ICU length of stay; vasopressor-free days; dialysis-free days

Outcomes relevant to this review: intubation rate; mortality; hospital length of stay; ICU length of stay

\begin{tabular}{ll}
\hline Starting date & 23 August 2018 \\
\hline Contact information & Israel Maia, israels.maia@gmail.com. Leticia Kawano-Dourado, Idourado@hcor.com.br \\
\hline
\end{tabular}

Notes

\section{NCT03788304}

Study name Methods Participants
High-flow nasal cannula versus non-invasive ventilation in prevention of escalation to invasive mechanical ventilation in patients with acute hypoxemic respiratory failure

$$
\text { RCT, parallel-group design }
$$

Estimated number of participants: 100

Setting: respiratory ICU, Egypt

Inclusion criteria: Admitted to the respiratory ICU with AHRF requiring NIV support; respiratory rate $>25$ breaths/minute; use of accessory muscles of respiration; paradoxical breathing; thoracoabdominal asynchrony; hypoxaemia evidenced by $\mathrm{PaO}_{2} / \mathrm{FiO}_{2}$ ratio $<300$

Exclusion criteria: indication for emergency endotracheal intubation; pulse < 50 breaths/min with decreased level of consciousness; persistent haemodynamic instability with systolic blood pressure $<90 \mathrm{mmHg}$ after infusing a bolus of crystalloid solution at a dose of $30 \mathrm{~mL} / \mathrm{kg}$ or life-threatening arrhythmia; undrained pneumothorax or pneumothorax with persistent air leak; extensive facial trauma or burn; refusal to participate; usual long-term treatment with NIV for chronic disease; altered mental status with decreased consciousness and/or evidence of inability to understand; tracheotomy or other upper airway disorders; active upper gastrointestinal bleeding

Interventions Intervention group (HFNC): flow $=30$ to $50 \mathrm{~L} / \mathrm{min}$

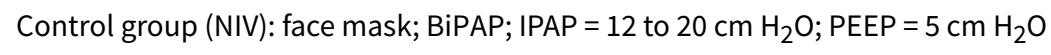

$\mathrm{FiO}_{2}$ adjusted to achieve $\mathrm{SpO}_{2}$ of $\geq 95 \%$ for both groups

Outcomes

All outcomes measured: intubation rate within 7 days; in-hospital mortality; length of ICU stay; duration of need for ventilatory support; development of complications due to devices

Outcomes relevant to this review: intubation rate within 7 days; in-hospital mortality; length of ICU stay; duration of need for ventilatory support; development of complications due to devices 
NCT03788304 (Continued)
Starting date
27th December 2018

Contact information

EntsarH Mohamed, dr.entsar_hsanen@yahoo.com.Gamal M Rabie gamalagmy135@gmail.com

Notes

\section{NCT03811158}

The diaphragm activity level and cardiopulmonary function between heated humidified high-flow
nasal cannula and unheated humidified high-flow oxygen mask in acute exacerbation of COPD pa-
tients as post-extubation respiratory support

\begin{tabular}{ll}
\hline Methods & RCT, cross-over design. Single-centre. No details of cross-over \\
\hline Participants & Estimated number of participants: 20 \\
& Setting: ICU, Taiwan \\
& Inclusion criteria: AECOPD; intubated and ready for weaning; $\mathrm{PaO}_{2} / \mathrm{FiO}_{2}>200$ during SBT \\
& $\begin{array}{l}\text { Exclusion criteria: unable to insert NG; refused reintubation; planned NIV post-extubation; preg- } \\
\text { nancy }\end{array}$ \\
\hline Interventions & $\begin{array}{l}\text { Intervention group (HFNC) } \\
\text { Control group (standard oxygen therapy): unheated humidified high-flow oxygen mask }\end{array}$ \\
\hline Outcomes & $\begin{array}{l}\text { All outcomes reported: diaphragm electrical activity; transcutaneous pulse oxymetry and capnog- } \\
\text { raphy; reintubation rate; cardiac index; hospital length of stay; ICU length of stay }\end{array}$ \\
\hline Outcomes relevant to this review: pulse oxymetry; reintubation rate; hospital length of stay
\end{tabular}

NCT03865056

Study name Therapy with high-flow oxygen by nasal cannula vs noninvasive ventilation in patients with acute
hypoxemic respiratory failure: a crossover physiologic study

\section{Methods}

Participants
RCT, cross-over design. Single-centre study. Interventions applied for 20 minutes in random order

Estimated number of participants: 20

Setting: ICU, Canada

Inclusion criteria: hypoxaemia; respiratory rate $>25$ breaths $/ \mathrm{min} ; \mathrm{PaCO}_{2} \leq 45 \mathrm{mmHg}$; absence of underlying chronic respiratory failure

Exclusion criteria: lack of consent; age < 18; invasive MV > 48 hrs; immediate need for intubation; previous inclusion in this study; systolic blood pressure $<90 \mathrm{mmHg}$; cardiogenic pulmonary oedema; GCS < 12; moribund; contraindications to NIV; tracheostomy 
NCT03865056 (Continued)

Interventions Intervention group (HFNC): Optiflow

Control group (NIV)

Outcomes

All outcomes measured: intra-tidal ventilation heterogeneity index; global inhomogeneity index; tidal volume; respiratory muscle effort; $\mathrm{SpO}_{2} ; \mathrm{PaO}_{2} / \mathrm{FiO}_{2} ; \mathrm{PaCO}_{2}$; respiratory rate

Outcomes relevant to this review: $\mathrm{SpO}_{2} ; \mathrm{PaO}_{2} / \mathrm{FiO}_{2} ; \mathrm{PaCO}_{2}$; respiratory rate

\begin{tabular}{ll}
\hline Starting date & 6 March 2019 \\
\hline Contact information & Lorenzo Del Sorbo, lorenzo.delsorbo@uhn.ca. Felicity Backhouse, felicity.backhouse@uhn.ca \\
\hline Notes & \\
\hline
\end{tabular}

\section{NCT03877172}

\begin{tabular}{ll}
\hline Study name & High-flow nasal cannula in thoracic surgery: a physiologic study \\
\hline Methods & RCT, cross-over design. Single-centre study. Randomized to intervention or control for 30 minutes \\
\hline Participants & Estimated number of patients: 40 \\
& Setting: postoperative ICU, Spain \\
& Inclusion criteria: lung resection with expected MV > 180 mins \\
& Exclusion criteria: refusal to participate; contraindications to NG tube placement; age < 18; preg- \\
nancy; neuromuscular disease; prior thoracic surgery
\end{tabular}

Interventions
Control group (standard oxygen therapy): conventional face mask
$\mathrm{FiO}_{2}$ titrated to $\mathrm{SpO}_{2}>92 \%$ for both groups

Outcomes

All outcomes reported: respiratory drive; thickening fraction of the right hemidiaphragm; diaphragmatic excursion; $\mathrm{PaO}_{2} / \mathrm{FiO}_{2} ; \mathrm{PaCO}_{2}$; dyspnoea

Outcomes relevant to this review: $\mathrm{PaO}_{2} / \mathrm{FiO}_{2} ; \mathrm{PaCO}_{2}$; dyspnoea

\begin{tabular}{ll}
\hline Starting date & 15 March 2019 \\
\hline Contact information & Ricard Mellado Artigas, rmartigas@gmail.com
\end{tabular}

Notes

\section{NCT03928535}

\section{Study name}

Effect of post-extubation high-flow nasal cannula vs noninvasive ventilation on reintubation and post-extubation respiratory failure in patients with hypercapnic COPD, a randomized controlled trial

Methods RCT, parallel-group design


NCT03928535 (Continued)

Participants
Estimated number of participants: 100

Setting: respiratory ICU, China

Inclusion Criteria: clinical diagnosis of AECOPD; $>48$ hrs MV; HFNC or NIV immediately after extubation; $\mathrm{PaCO}_{2} \geq 50 \mathrm{mmHg}$ at point of extubation; PEEP $\leq 8 \mathrm{~cm} \mathrm{H}_{2} \mathrm{O}$ at extubation

Exclusion Criteria: do-not-intubate; tracheostomies; accidental extubation; self-extubation
Intervention group (HFNC): flow initially $10 \mathrm{~L} / \mathrm{min}$ and titrated up in $5 \mathrm{~L} / \mathrm{min}$ steps until intolerable; $\mathrm{FiO}_{2}$ was titrated to target $\mathrm{SpO}_{2}>92 \%$

Control group (NIV): BiPAP for 24 hours then oxygen via Venturi mask. PEEP, IPAP and $\mathrm{FiO}_{2}$ were adjusted to achieve respiratory rate $<25$ breaths $/ \mathrm{min}$ and $\mathrm{SpO}_{2}>92 \%$

Outcomes $\quad$ All outcomes measured: rate of reintubation within 72 hours; ICU length of stay; 28-day mortality; $\mathrm{PaCO}_{2}$

Outcomes relevant to this review: rate of reintubation; ICU length of stay; 28-day mortality; $\mathrm{PaCO}_{2}$

\begin{tabular}{ll}
\hline Starting date & 26th April 2019 \\
\hline Contact information & Not available \\
\hline Notes & Not yet recruiting \\
\hline
\end{tabular}

\section{NCT03944525}

\begin{tabular}{ll}
\hline Study name & High-flow air via nasal cannula versus non-invasive continuous positive airway pressure ventilation \\
support for hypercapnic respiratory failure the HIGH-for-HYPER study
\end{tabular}
support for hypercapnic respiratory failure the HIGH-for-HYPER study

\begin{tabular}{|c|c|}
\hline Methods & $\mathrm{RCT}$, parallel-group design. Single-centre study \\
\hline \multirow[t]{4}{*}{ Participants } & Estimated number of participants: 62 \\
\hline & Setting: ICU beds within Emergency Department, Austria \\
\hline & $\begin{array}{l}\text { Inclusion criteria: age > 18; treated at the Emergency Department; acute hypercapnic respiratory } \\
\text { failure defined as a } \mathrm{PaCO}_{2}>50 \mathrm{mmHg} \text { and a } \mathrm{pH}<7.30 \text { on admission }\end{array}$ \\
\hline & $\begin{array}{l}\text { Exclusion criteria: comatose on admission; no intact airway; lack of airway-protective reflexes; not } \\
\text { alert enough to follow commands; patients intubated by Emergency Medical Service; patients re- } \\
\text { quiring intubation on admission; pregnant women }\end{array}$ \\
\hline \multirow[t]{3}{*}{ Interventions } & Intervention group $(\mathrm{HFNC})$ : flow $=60 \mathrm{~L} / \mathrm{min} ; \mathrm{FiO}_{2}$ titrated to clinical need \\
\hline & Control group (NIV): CPAP; face mask; PEEP $=5 \mathrm{~cm} \mathrm{H}_{2} \mathrm{O} ; \mathrm{FiO}_{2}$ titrated to clinical need \\
\hline & $\begin{array}{l}\text { Both therapies were continued until } \mathrm{PCO}_{2} \text { level of } \leq 50 \mathrm{mmHg} \text { reached, therapy aborted due to in- } \\
\text { tolerance, or indication for intubation }\end{array}$ \\
\hline Outcomes & $\begin{array}{l}\text { All outcomes measured: change in } \mathrm{PCO}_{2} \text {; frequency of therapy failure (intubation); patient's per- } \\
\text { ception of therapy; rate of adverse events; time to } \mathrm{PCO}_{2} \leq 50 \mathrm{mmHg} \text {; length of stay in the ED; admis- } \\
\text { sion to ICU; admission to regular ward; length of ICU stay; length of hospital stay; hospital re-admis- } \\
\text { sion within } 30 \text { days }\end{array}$ \\
\hline
\end{tabular}


NCT03944525 (Continued)

Outcomes relevant to this review: change in $\mathrm{PCO}_{2}$; frequency of therapy failure (intubation); patient's perception of therapy; rate of adverse events; length of hospital stay; length of ICU stay

\begin{tabular}{ll}
\hline Starting date & 9th May 2019 \\
\hline Contact information & Verena Fuhrmann, verena.fuhrmann@meduniwien.ac.at \\
\hline Notes & $\begin{array}{l}\text { This study was carried out in an Emergency Department with in-department ICU beds where the in- } \\
\text { tervention was given. We decided to include this study for this reason. }\end{array}$ \\
\hline
\end{tabular}

\section{NCT04035460}

Study name

A pilot randomized controlled study of non-invasive oxygenation and ventilation in patients with acute hypoxemic respiratory failure (AHRF): a comparison of oxygen delivery via helmet interface versus high-flow nasal cannula (HFNC)

\begin{tabular}{ll}
\hline Methods & RCT, parallel-group design \\
\hline Participants & Estimated number of participants: 40 \\
& Setting: USA \\
& Inclusion criteria: age $\geq 18$ years; respiratory rate $\geq 24 /$ min and/or subjective shortness of breath; \\
& AHRF \\
& Exclusion criteria: severe ARDS; $>24$ hours since meeting criteria for AHRF; urgent need for intu- \\
& bation; contraindication to HFNC, Helmet-NIPPV, or Mask-NIPPV; upper airway obstruction; facial \\
& trauma; copious secretions, airway bleeding, epistaxis or vomiting; primary cause of respiratory \\
& failure is AECOPD or asthma; elevated intracranial pressure $>20$ mmH; home mechanical ventila- \\
& tion except for CPAP/BiPAP used solely for sleep-disordered breathing; persistent haemodynamic \\
& instability; plan for procedure during which NIPPV or HFNC is contraindicated; absence of airway \\
protective gag reflex or cough; tracheostomy; lack of informed consent; pregnancy; actual body & weight exceeding 1 kg per cm of height; diffuse alveolar haemorrhage; severe acute pancreatitis \\
as etiology for hypoxaemia; recent upper gastrointestinal surgical anastomosis within the past 30 \\
days; enrolment in another clinical trial within the past 30 days; unsuitable for NIV in the judgment \\
of the treating physician; decision to withhold life-sustaining treatment (Patients with Do-Not-Re- \\
suscitate (DNR) or No-Cardiopulmonary-Resuscitation (No CPR) order may be enrolled); do-not-in- \\
tubate order
\end{tabular}

Interventions Intervention group (HFNC)

Control group (NIV): via helmet

Outcomes

All outcomes measured: rate of intubation within 28 days; time to intubation within 28 days; intubation-free days within 28 days; organ-failure-free days within 28 days; mortality prior to discharge or study day 90; hospital mortality to day 28 ; ICU-free days within 28 days; hospital length of stay; rate of cross-over between groups or to other forms of NIV; complication rate; total daily dose of sedative medications within 7 days; highest level of daily mobility within 7 days; tolerance of devices; rate and reason for exclusion from enrolment through study completion; rate of intubation in non-enrolled patients that meet inclusion and exclusion criteria within 28 days; RASS within 7 days; Confusion Assessment Method for the ICU within 7 days

Outcomes relevant to this review: rate of intubation within 28 days; mortality prior to discharge or study day 90; hospital mortality to day 28; hospital length of stay; rate of cross-over between groups or to other forms of NIV; complication rate; tolerance of devices 
NCT04035460 (Continued)

Contact information

Mark A Tidswell,mark.tidswell@baystatehealth.org.Cynthia Kardos, cynthia.kardos@baystatehealth.org

Notes

NCT04036175

$\begin{array}{ll}\text { Study name } & \text { Comparison of patient work of breathing and tidal volumes with high-flow nasal cannula oxygen } \\ \text { therapy and NIV (non-invasive ventilation) after extubation in the ICU }\end{array}$

Methods RCT, cross-over design. Interventions delivered in a random order for 20 minutes each

Participants

Estimated number of participants: 25

Setting: ICU, France

Inclusion Criteria: $\geq 18$ years of age; planned extubation decided by the physician in charge of the participant after success of weaning trial; at high risk of reintubation ( $>65$ years of age; underlying chronic cardiac or lung disease); hypoxaemia $\left(\mathrm{PaO}_{2} / \mathrm{FiO}_{2}<300 \mathrm{mmHg}\right.$ under $\mathrm{MV}$ before extubation)

Exclusion Criteria: duration of MV prior to extubation < 24 hours; contraindication to NIV; contraindication to nasogastric tube; do-not-reintubate order at time of extubation; under legal protection; opposition to participate

Interventions Intervention group (HFNC): flow $=50 \mathrm{~L} / \mathrm{min}$

Control group (NIV): BiPAP; PEEP $=5 \mathrm{~cm} \mathrm{H}_{2} \mathrm{O}$, IPAP adjusted to achieve expired tidal volume of 6 to $8 \mathrm{~mL} / \mathrm{kg}$

$\mathrm{FiO}_{2}$ adjusted to obtain $\mathrm{SpO}_{2} \geq 92 \%$ for both groups

Outcomes

All outcomes measured: patient respiratory effort and tidal volume; respiratory rate; $\mathrm{SpO}_{2}$; transcutaneous $\mathrm{PaCO}_{2}$; transpulmonary pressure; systolic and diastolic arterial pressure; comfort evaluation (visual evaluation scale)

Outcomes relevant to this review: patient respiratory effort; respiratory rate; $\mathrm{SpO}_{2}$; transcutaneous $\mathrm{PaCO}_{2}$; comfort level

\begin{tabular}{ll}
\hline Starting date & 6 March 2018 \\
\hline Contact information & Arnaud W Thille, arnaud.thille@chu-poitiers.fr \\
\hline Notes & \\
\hline
\end{tabular}

\section{NCT04156139}

Study name

High-flow nasal cannula versus noninvasive positive pressure ventilation therapy after early extubation for patients with chronic obstructive pulmonary disease

\begin{tabular}{ll}
\hline Methods & RCT, parallel-group design \\
\hline Participants & Estimated number of participants: 502 \\
& Setting: ICU, China
\end{tabular}


NCT04156139 (Continued)

Inclusion Criteria: age $=40$ to 85 years; COPD patients with bronchopulmonary infection; patients with hypercapnic respiratory failure treated with invasive mechanical ventilation for between 48 hours and 14 days; reached the pulmonary infection control window; have self-care ability with oxygen supply during stable phase

Exclusion Criteria: severe organ dysfunction; myopathy or myasthenia gravis; upper airway obstruction; a large amount of secretions and inability to drain

Interventions Intervention group (HFNC): flow rate $=45$ to $55 \mathrm{~L} / \mathrm{min} ; \mathrm{FiO}_{2}$ adjusted to maintain $\mathrm{SpO}_{2} \geq 92 \%$

Control group (NIV): BiPAP; initial PEEP $=5 \mathrm{~cm} \mathrm{H}_{2} \mathrm{O}$; IPAP $=10 \mathrm{~cm} \mathrm{H}_{2} \mathrm{O}$; adjusted to target tidal volume of 6-8 $\mathrm{mL} / \mathrm{kg}$

Outcomes All outcomes measured: reintubation within 7 days; weaning failure within 7 days (composite criterion including reintubation and all-cause mortality)

Outcomes relevant to this review: reintubation, all-cause mortality

\begin{tabular}{ll}
\hline Starting date & $7^{\text {th }}$ November 2019 \\
\hline Contact information & Han Xiaobo, hansir510@163.com \\
\hline
\end{tabular}

Notes

NCT04241861

Study name

Physiological comparison of high-flow nasal cannula, helmet pressure support ventilation and continuous positive airway pressure during acute hypoxemic respiratory failure: a randomized crossover study

\begin{tabular}{|c|c|}
\hline Methods & $\mathrm{RCT}$, cross-over design. Interventions delivered in a random order for 40 minutes each \\
\hline \multirow[t]{5}{*}{ Participants } & Number of estimated participants: 15 \\
\hline & Setting: emergency department or ICU, Italy \\
\hline & $\begin{array}{l}\text { Inclusion criteria: adult hypoxemic non-hypercapnic participants admitted to the emergency de- } \\
\text { partment or the ICU with de novo AHRF; respiratory rate }>25 \text { breaths } / \mathrm{min} ; \mathrm{PaO}_{2} / \mathrm{FiO}_{2} \leq 200 ; \mathrm{PaCO}_{2}\end{array}$ \\
\hline & $\begin{array}{l}<45 \mathrm{mmHg} \text {; absence of history of chronic respiratory failure or moderate to severe cardiac insuffi- } \\
\text { ciency; written informed consent }\end{array}$ \\
\hline & $\begin{array}{l}\text { Exclusion criteria: exacerbation of asthma or COPD; cardiogenic pulmonary oedema; haemody- } \\
\text { namic instability; lactic acidosis (lactate }>5 \mathrm{mmol} / \mathrm{L}) \text {; clinically diagnosed shock; metabolic acido- } \\
\text { sis ( } \mathrm{pH}<7.30 \text { with normal- or hypo-carbia); GCS }<13 \text {; recent head surgery or anatomy that prevents } \\
\text { the application of helmet or nasal cannula to patient's face }\end{array}$ \\
\hline
\end{tabular}

Interventions Intervention group (HFNC): initial flow $\geq 50 \mathrm{~L} / \mathrm{min}$, decreased in case of intolerance to $\geq 30 \mathrm{~L} / \mathrm{min}$

Control group 1 (NIV): helmet PSV; BiPAP; IPAP $\geq 8$ to $10 \mathrm{~cm} \mathrm{H}_{2} \mathrm{O}$ to permit inspiratory flow of $100 \mathrm{~mL} / \mathrm{min} ; \mathrm{PEEP}=10$ to $12 \mathrm{~cm} \mathrm{H}_{2} \mathrm{O}$ increasing to achieve oxygenation target as required

Control group 2 (NIV): helmet CPAP; continuous airflow $=50$ to $60 \mathrm{~L} / \mathrm{min}$. $\mathrm{PEEP}=10$ to $12 \mathrm{~cm} \mathrm{H}_{2} \mathrm{O}$ increasing to achieve oxygenation target as required

$\mathrm{FiO}_{2}$ will be titrated to obtain $\mathrm{SpO}_{2}$ of $92-98 \%$ for all groups

Outcomes

All outcomes measured: inspiratory effort; tidal volume; oxygenation; tidal volume distribution; $\mathrm{PaCO}_{2}$; dyspnoea; comfort; global and regional impedance-derived end-expiratory lung volumes 
NCT04241861 (Continued)

and dynamic strains; dynamic transpulmonary driving pressure; respiratory system dynamic compliance; pendelluft; work of breathing

Outcomes relevant to this review: oxygenation; work of breathing; dyspnoea; comfort; $\mathrm{PaCO}_{2}$

\begin{tabular}{ll}
\hline Starting date & 27th January 2020 \\
\hline Contact information & Domenico L Grieco, dlgrieco@outlook.it \\
\hline Notes & \\
\hline
\end{tabular}

\section{NCT04253405}

Study name

Multicentric randomized controlled pilot study comparing high-flow nasal cannula versus non-invasive positive pressure ventilation in acute respiratory failure in patients with pulmonary fibrosis (RENOVATE Fibrosis)

\begin{tabular}{ll}
\hline Methods & RCT, parallel-group design. Multicentre study \\
\hline Participants & Estimated number of participants: 50 \\
& Setting: ICU or ED, Brazil \\
& Inclusion criteria: age $\geq 18 ;$ admitted to ICU or ED with pulmonary fibrosis and AHRF \\
& Exclusion criteria: pulmonary fibrosis secondary to progressive massive fibrosis (silicosis), or any \\
& other tumour form of fibrosis; significant pulmonary arterial hypertension; pneumothorax or ex- \\
& tensive pleural effusion; cardiogenic pulmonary congestion; delirium or non-co-operation at the \\
& time of randomization; facial abnormalities; uncoercible vomiting or hypersecretion of the airways; \\
use of continuous NIPPV or HFNC for more than 8 hours before randomization; pregnancy; refusal \\
to participate
\end{tabular}

Interventions

Intervention group (HFNC): Airvo2, Fisher \& Paykel

Control group (NIV): BiPAP; oronasal or full face mask

\section{Outcomes}

All outcomes measured: recruitment feasibility; dyspnoea (Borg scale); respiratory rate; oxygenation; $\mathrm{PaCO}_{2}$

Outcomes relevant to this review: dyspnoea (Borg scale); respiratory rate; oxygenation; $\mathrm{PaCO}_{2}$

\begin{tabular}{ll}
\hline Starting date & 5 February 2020 \\
\hline Contact information & Leticia Kawano-Dourado, Idourado@hcor.com.br. Karina Negrelli, knegrelli@hcor.com.br \\
\hline Notes & \\
\hline
\end{tabular}

\section{NCT04269681}

Study name

Renovate palliative study: randomized controlled trial comparing high-flow nasal catheter versus standard respiratory support in patients with do-not-intubate order and acute respiratory failure

\begin{tabular}{ll}
\hline Methods & RCT, parallel-group design. Multicentre study \\
\hline Participants & Estimated number of participants: 150
\end{tabular}


NCT04269681 (Continued)

\section{Setting: ICU, Brazil}

Inclusion criteria: age $\geq 18$; AHRF of any cause on admission or post-extubation; do-not-intubate order; dyspnoea (Borg scale $\geq 4$ ); $\mathrm{SpO}_{2}<90 \%$ or $\mathrm{PaO}_{2}<60 \mathrm{mmHg}$ on room air; absence of delirium; signs of respiratory distress and use of accessory muscles or respiratory rate $>25$ breaths $/ \mathrm{min}$

Exclusion criteria: refusal of treatment; agitation or non-co-operation; delirium at randomization; anatomical abnormalities that would interfere with NIV mask; GCS $<12$; psychomotor agitation requiring sedation; contraindications to NIV; pneumothorax or extensive pleural effusion; moribund

Interventions Intervention group (HFNC): AIRVO 2, Fisher \& Paykel Healthcare, Auckland, New Zealand; offered until resolution of AHRF or intolerance; flow $=45 \mathrm{~L} / \mathrm{min}$ titrated to $60 \mathrm{~L} / \mathrm{min}$ or highest tolerable flow; $\mathrm{FiO}_{2}=0.5$ titrated to $\mathrm{SpO}_{2}=92$ to $98 \%$

Control group (standard oxygen therapy): $\mathrm{SpO}_{2}$ targeted 90-98\%; NIV at discretion of the treatment team

\begin{tabular}{ll}
\hline Outcomes & $\begin{array}{l}\text { All outcomes reported: dyspnoea; comfort; opioid dose; delirium; ICU length of stay; mortality (28 } \\
\text { day); use of respiratory support devices } \\
\text { Outcomes relevant to this review: comfort; ICU length of stay; mortality; use of respiratory support } \\
\text { devices (if NIV/MV) }\end{array}$ \\
\hline Starting date & 17 February 2020 \\
\hline Contact information & Israel Maia, israils.maia@gmail.com. Leticia Kawano-Dourado, Idourado@hcor.com.br \\
\hline Notes & \\
\hline
\end{tabular}

NCT04293991

Study name

High-flow nasal cannula versus non-invasive ventilation in prevention of intubation in immunocompromised patient with acute hypoxemic respiratory failure

Methods RCT, parallel-group design

Participants

Estimated number of participants: 76

Setting: ICU, Egypt

Inclusion criteria: admitted immunocompromised patient to ICU with AHRF; haematological malignancies; post-bone marrow transplantation

Exclusion criteria: need of emergency intubation; patient with deterioration of conscious level with hypoxaemia with $\mathrm{FiO}_{2}$ less than $90 \%$ in spite of maximum $\mathrm{O}_{2}$ support; haemodynamic instability with need of vasoconstrictor support

Interventions

Intervention group (HFNC): flow $=60 \mathrm{~L} / \mathrm{min}$ and titrated downwards to comfort; patient encouraged to have mouth closed

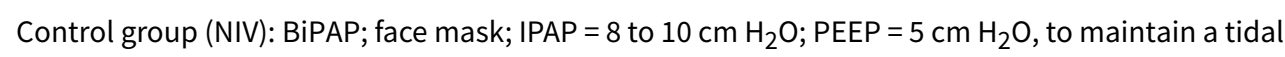
volume of $6-8 \mathrm{~mL} / \mathrm{kg}$

$\mathrm{FiO}_{2}$ titrated to $\mathrm{SpO}_{2}$ of $\geq 92 \%$ for both groups

\section{Outcomes}

All outcomes measured: intubation within 48 hours of admission; 28-day mortality

Outcomes relevant to this review: intubation within 48 hours of admission; 28-day mortality 
NCT04293991 (Continued)

Starting date 3rd March 2020

Contact information

Ashraf Elagamy, elagamy_ashraf@yahoo.com. Dalia Elfawy, daliamfawy@yahoo.com

Notes

NCT04344730

Study name Dexamethasone and oxygen support strategies in ICU patients with Covid-19 pneumonia (COVIDICUS trial)

\begin{tabular}{ll}
\hline Methods & RCT, $2 \times 2$ factorial design \\
\hline Participants & Estimated number of participants: 550 \\
& Setting: ICU, France \\
& Inclusion criteria: age $\geq 18$ years; admitted to ICU within 48 hours; confirmed or highly suspected \\
& Covid-19 infection; AHRF; any treatment intended to treat the SARS-CoV-2 infection (compassion- \\
ate or in context of clinical trial) & \\
& $\begin{array}{l}\text { Exclusion criteria: moribund; pregnancy or breastfeeding; long-term corticotherapy; active and } \\
\text { untreated bacterial, fungal or parasitic infection; no written informed consent; hypersensitivity to } \\
\text { dexamethasone; not affiliated to French social security; anatomical factors precluding use of nasal } \\
\text { cannula; hypercapnia indicating NIV }\end{array}$
\end{tabular}

Interventions Intervention group (HFNC): flow $=30 \mathrm{~L} / \mathrm{min} ; \mathrm{FiO}_{2}$ adjusted to target $\mathrm{SpO}_{2} \geq 92 \%$

Control group (NIV): CPAP; flow adjusted to target $\mathrm{SpO}_{2} \geq 92 \%$

Outcomes All outcomes measured: time-to-death; time-to-MV; viral load of SARS-CoV-2 in respiratory tract; healthcare-associated infection rate; days alive without MV; SOFA score; days alive without renal replacement therapy; length of ICU stay; length of hospital stay; number of patients with severe hypoxaemia $\left(\mathrm{SpO}_{2}<80 \%\right)$; cardiac arrest within 1 hour of intubation

Outcomes relevant to this review: length of ICU stay; length of hospital stay; adverse event rate

\begin{tabular}{ll}
\hline Starting date & 14 April 2020 \\
\hline Contact information & Jean François, jean-françois.timsit@aphp.fr. Lila Bouadma, lila.bouadma@aphp.fr \\
\hline Notes & \\
\hline
\end{tabular}

\section{TCTR20171106003}

Study name

High-flow nasal oxygen for prevention of intubation in acute non-hypercapnic hypoxemic respiratory failure in immunocompromised patients, a randomized trial

Methods RCT, parallel-group design

Participants

Estimated number of participants: 74

Setting: Thailand 
Inclusion criteria: immunocompromised patients; solid or haematologic malignancy; received immunosuppressive drug or steroid at a dose $>20 \mathrm{mg} /$ day of prednisolone for $>30$ days; HIV infection; require nasal low-flow oxygen of $4 \mathrm{~L} / \mathrm{min}$ to maintain $\mathrm{PaO}_{2}$ above $60 \mathrm{mmHg}$ or $\mathrm{SpO}_{2}>90 \%$ for $>1$ hour; age $\geq 18$ years

Exclusion criteria: $\mathrm{PaCO}_{2}>45 \mathrm{mmHg}$; post-extubation respiratory failure (respiratory failure within $48 \mathrm{hr}$ after extubation); life-threatening AHRF requiring immediate invasive MV; shock; active pulmonary tuberculosis; contraindications to NIV; do-not-intubate order; patients refused to participate in the study

\begin{tabular}{ll}
\hline Interventions & Intervention group (HFNC) \\
& Control group (NIV)
\end{tabular}

Control group (NIV)

Outcomes All outcomes measured: required intubation and invasive mechanical ventilation within $48 \mathrm{hrs;} 30$ day mortality rate

Outcomes relevant to this review: required intubation and invasive mechanical ventilation within 48 hrs; 30-day mortality rate

\begin{tabular}{ll}
\hline Starting date & $7^{\text {th }}$ August 2017 \\
\hline Contact information & Juthamas Inchai, juinchai@gmail.com \\
\hline Notes & \\
\hline
\end{tabular}

\section{UMIN000008778}

\begin{tabular}{|c|c|}
\hline Study name & Evaluation of nasal high-flow oxygen therapy for severe acute hypoxaemic respiratory failure \\
\hline Methods & $\mathrm{RCT}$, parallel-group design \\
\hline \multirow[t]{4}{*}{ Participants } & Estimated number of participants: 40 \\
\hline & Setting: respiratory department, Japan \\
\hline & $\begin{array}{l}\text { Inclusion criteria: admitted to respiratory department for severe AHRF other than cardiogenic pul- } \\
\text { monary oedema; met the standard clinical and/or blood gas criteria for use of NIV to treat severe } \\
\text { AHRF; received NIV for }<12 \text { hours }\end{array}$ \\
\hline & $\begin{array}{l}\text { Exclusion criteria: } \mathrm{PaCO}_{2}>45 \mathrm{mmHg} \text {; unstable clinical conditions (i.e. need for vasopressors, meta- } \\
\text { bolic acidosis, life-threatening arrhythmias, need for } \mathrm{FiO}_{2} \geq 0.8 \text {, agitation and anxiety); inability to } \\
\text { obtain consent; face or neck deformities; use of NIV before admission; need for continuous seda- } \\
\text { tion }\end{array}$ \\
\hline Interventions & $\begin{array}{l}\text { Intervention group (HFNC) } \\
\text { Control group (NIV) }\end{array}$ \\
\hline \multirow[t]{2}{*}{ Outcomes } & $\begin{array}{l}\text { All outcomes measured: interface discomfort; dyspnoea; ease of speaking; sleep perception; easy } \\
\text { to eat and drink; ABG; vital signs; early failure; length of ICU stay; length of hospital stay; hospital } \\
\text { mortality; 90-day survival; complications }\end{array}$ \\
\hline & $\begin{array}{l}\text { Outcomes relevant to this review: discomfort; dyspnoea; ABG; vital signs; early failure; length of } \\
\text { ICU stay; length of hospital stay; hospital mortality; complications }\end{array}$ \\
\hline Starting date & September 2012 \\
\hline
\end{tabular}


UMIN000008778 (Continued)

AECOPD: acute exacerbation of COPD

AFOT: automated flow oxygen titration

AHRF: acute hypoxic respiratory failure

AIDS: acquired immune deficiency syndrome

APACHE: Acute Physiology and Chronic Health Evaluation

ARDS: acute respiratory distress syndrome

BiPAP: bilevel positive airway pressure

BMI: body mass index

COPD: chronic obstructive pulmonary disease

COVID-19: coronavirus disease 19

CPAP: continuous positive airway pressure

ED: emergency department

EIT: electrical impedance tomography

$\mathrm{FiO}_{2}$ : fraction of inspired oxygen

GCS: Glasgow coma score

HFNC: high-flow nasal cannulae

HFNO: high-flow nasal oxygen

HFO: high-flow oxygen

hrs: hours

ICU: intensive care unit

IPAP: inspiratory positive airway pressure

MV: mechanical ventilation

NG: nasogastric

NIV: non-invasive ventilation

NPPV: see NIV

OSA: obstructive sleep apnoea

$\mathrm{PaCO}_{2}$ : carbon dioxide clearance

$\mathrm{PaO}_{2}$ : partial pressure of arterial oxygen

$\mathrm{PCO}_{2}$ : partial pressure of carbon dioxide

PEEP: positive end expiratory pressure

PIC: pulmonary infection control

PSV: pressure support ventilation

RASS: Richmond agitation and sedation score

$\mathrm{RCT}$ : randomized controlled trial

REM: rapid eye movement

$\mathrm{SaO} 2$ : oxygen saturation

SARS-CoV-2: severe acute respiratory syndrome coronavirus 2

SBT: spontaneous breathing trial

SOFA: sequential organ failure assessment score

$\mathrm{SpO}_{2}$ : oxygen saturation

$\mathrm{S} / \mathrm{T}$ : spontaneous/timed

\section{DATA AND ANALYSES}


Comparison 1. HFNC versus standard oxygen therapy

\begin{tabular}{|c|c|c|c|c|}
\hline Outcome or subgroup title & No. of studies & $\begin{array}{l}\text { No. of partici- } \\
\text { pants }\end{array}$ & Statistical method & Effect size \\
\hline $\begin{array}{l}1.1 \text { Treatment failure (escalation of } \\
\text { respiratory support to NIV, NIPPV } \\
\text { or invasive ventilation) }\end{array}$ & 15 & 3044 & $\begin{array}{l}\text { Risk Ratio (M-H, Random, 95\% } \\
\text { Cl) }\end{array}$ & $0.62[0.45,0.86]$ \\
\hline $\begin{array}{l}\text { 1.1.1 Post-extubation respiratory } \\
\text { support }\end{array}$ & 11 & 1912 & $\begin{array}{l}\text { Risk Ratio (M-H, Random, 95\% } \\
\text { Cl) }\end{array}$ & $0.50[0.30,0.86]$ \\
\hline $\begin{array}{l}\text { 1.1.2 Respiratory support without } \\
\text { prior use of mechanical ventilation }\end{array}$ & 4 & 1132 & $\begin{array}{l}\text { Risk Ratio (M-H, Random, 95\% } \\
\mathrm{Cl} \text { ) }\end{array}$ & $0.85[0.68,1.08]$ \\
\hline 1.2 In-hospital mortality & 11 & 2673 & $\begin{array}{l}\text { Risk Ratio (M-H, Random, 95\% } \\
\text { Cl) }\end{array}$ & $0.96[0.82,1.11]$ \\
\hline 1.3 Important adverse events & 5 & & $\begin{array}{l}\text { Risk Ratio (M-H, Random, 95\% } \\
\mathrm{Cl} \text { ) }\end{array}$ & Subtotals only \\
\hline 1.3.1 Pneumonia & 4 & 1057 & $\begin{array}{l}\text { Risk Ratio (M-H, Random, 95\% } \\
\mathrm{Cl} \text { ) }\end{array}$ & $0.72[0.48,1.09]$ \\
\hline 1.3.2 Nasal mucosa or skin trauma & 2 & 617 & $\begin{array}{l}\text { Risk Ratio (M-H, Random, 95\% } \\
\mathrm{Cl} \text { ) }\end{array}$ & $3.66[0.43,31.48]$ \\
\hline 1.4 Length of ICU stay (days) & 6 & 970 & $\begin{array}{l}\text { Mean Difference (IV, Random, } \\
95 \% \mathrm{CI} \text { ) }\end{array}$ & $0.13[-0.02,0.28]$ \\
\hline $\begin{array}{l}1.5 \text { Short-term respiratory effects: } \\
\mathrm{PaO}_{2} / \mathrm{FiO}_{2}(\mathrm{mmHg})\end{array}$ & 5 & 600 & $\begin{array}{l}\text { Mean Difference (IV, Random, } \\
95 \% \mathrm{CI})\end{array}$ & $10.34[-17.31,38.00]$ \\
\hline 1.6 Comfort & 4 & & $\begin{array}{l}\text { Mean Difference (IV, Random, } \\
95 \% \mathrm{CI})\end{array}$ & Subtotals only \\
\hline 1.6.1 Short-term effect & 4 & 662 & $\begin{array}{l}\text { Mean Difference (IV, Random, } \\
95 \% \mathrm{CI})\end{array}$ & $0.31[-0.60,1.22]$ \\
\hline 1.6.2 Long-term effect & 2 & 445 & $\begin{array}{l}\text { Mean Difference (IV, Random, } \\
95 \% \mathrm{CI})\end{array}$ & $0.59[-2.29,3.47]$ \\
\hline $\begin{array}{l}\text { 1.7 Long-term respiratory effects: } \\
\mathrm{PaO}_{2} / \mathrm{FiO}_{2}(\mathrm{mmHg})\end{array}$ & 2 & 195 & $\begin{array}{l}\text { Mean Difference (IV, Random, } \\
95 \% \mathrm{CI})\end{array}$ & $34.28[-19.25,87.80]$ \\
\hline $\begin{array}{l}\text { 1.8 Short-term and long-term res- } \\
\text { piratory effects: } \mathrm{PaO}_{2}(\mathrm{mmHg})\end{array}$ & 5 & & $\begin{array}{l}\text { Mean Difference (IV, Random, } \\
95 \% \mathrm{CI})\end{array}$ & Subtotals only \\
\hline 1.8.1 Short-term effects & 4 & 415 & $\begin{array}{l}\text { Mean Difference (IV, Random, } \\
95 \% \mathrm{CI} \text { ) }\end{array}$ & $4.92[-1.24,11.07]$ \\
\hline 1.8.2 Long-term effects & 2 & 644 & $\begin{array}{l}\text { Mean Difference (IV, Random, } \\
95 \% \mathrm{CI} \text { ) }\end{array}$ & $12.27[7.51,17.04]$ \\
\hline $\begin{array}{l}1.9 \text { Short-term and long-term res- } \\
\text { piratory effects: } \mathrm{SpO}_{2}(\%)\end{array}$ & 5 & & $\begin{array}{l}\text { Mean Difference (IV, Random, } \\
95 \% \mathrm{CI})\end{array}$ & Subtotals only \\
\hline
\end{tabular}




\begin{tabular}{|c|c|c|c|c|}
\hline Outcome or subgroup title & No. of studies & $\begin{array}{l}\text { No. of partici- } \\
\text { pants }\end{array}$ & Statistical method & Effect size \\
\hline 1.9.1 Short-term effects & 5 & 572 & $\begin{array}{l}\text { Mean Difference (IV, Random, } \\
95 \% \mathrm{CI})\end{array}$ & $0.79[-0.29,1.88]$ \\
\hline 1.9.2 Long-term effects & 2 & 445 & $\begin{array}{l}\text { Mean Difference (IV, Random, } \\
95 \% \mathrm{CI} \text { ) }\end{array}$ & $1.28[0.02,2.55]$ \\
\hline $\begin{array}{l}1.10 \text { Short-term respiratory effects: } \\
\mathrm{PaCO}_{2}(\mathrm{mmHg})\end{array}$ & 5 & 755 & $\begin{array}{l}\text { Mean Difference (IV, Random, } \\
95 \% \mathrm{CI})\end{array}$ & $-1.05[-2.24,0.13]$ \\
\hline $\begin{array}{l}1.11 \text { Short-term and long-term res- } \\
\text { piratory rate (breaths/min) }\end{array}$ & 9 & 1608 & $\begin{array}{l}\text { Mean Difference (IV, Random, } \\
95 \% \mathrm{Cl} \text { ) }\end{array}$ & $-2.01[-3.19,-0.83]$ \\
\hline 1.11.1 Short-term effects & 8 & 1017 & $\begin{array}{l}\text { Mean Difference (IV, Random, } \\
95 \% \mathrm{Cl} \text { ) }\end{array}$ & $-2.02[-3.66,-0.37]$ \\
\hline 1.11.2 Long-term effects & 4 & 591 & $\begin{array}{l}\text { Mean Difference (IV, Random, } \\
95 \% \mathrm{CI} \text { ) }\end{array}$ & $-2.01[-4.39,0.37]$ \\
\hline 1.12 Length of hospital stay (days) & 2 & 450 & $\begin{array}{l}\text { Mean Difference (IV, Random, } \\
95 \% \mathrm{Cl})\end{array}$ & $-0.11[-0.43,0.20]$ \\
\hline $\begin{array}{l}1.13 \text { Refusal to continue with treat- } \\
\text { ment }\end{array}$ & 2 & 560 & $\begin{array}{l}\text { Risk Ratio (M-H, Random, 95\% } \\
\mathrm{Cl} \text { ) }\end{array}$ & $26.89[3.67,197.32]$ \\
\hline
\end{tabular}


Analysis 1.1. Comparison 1: HFNC versus standard oxygen therapy, Outcome 1: Treatment failure (escalation of respiratory support to NIV, NIPPV or invasive ventilation)

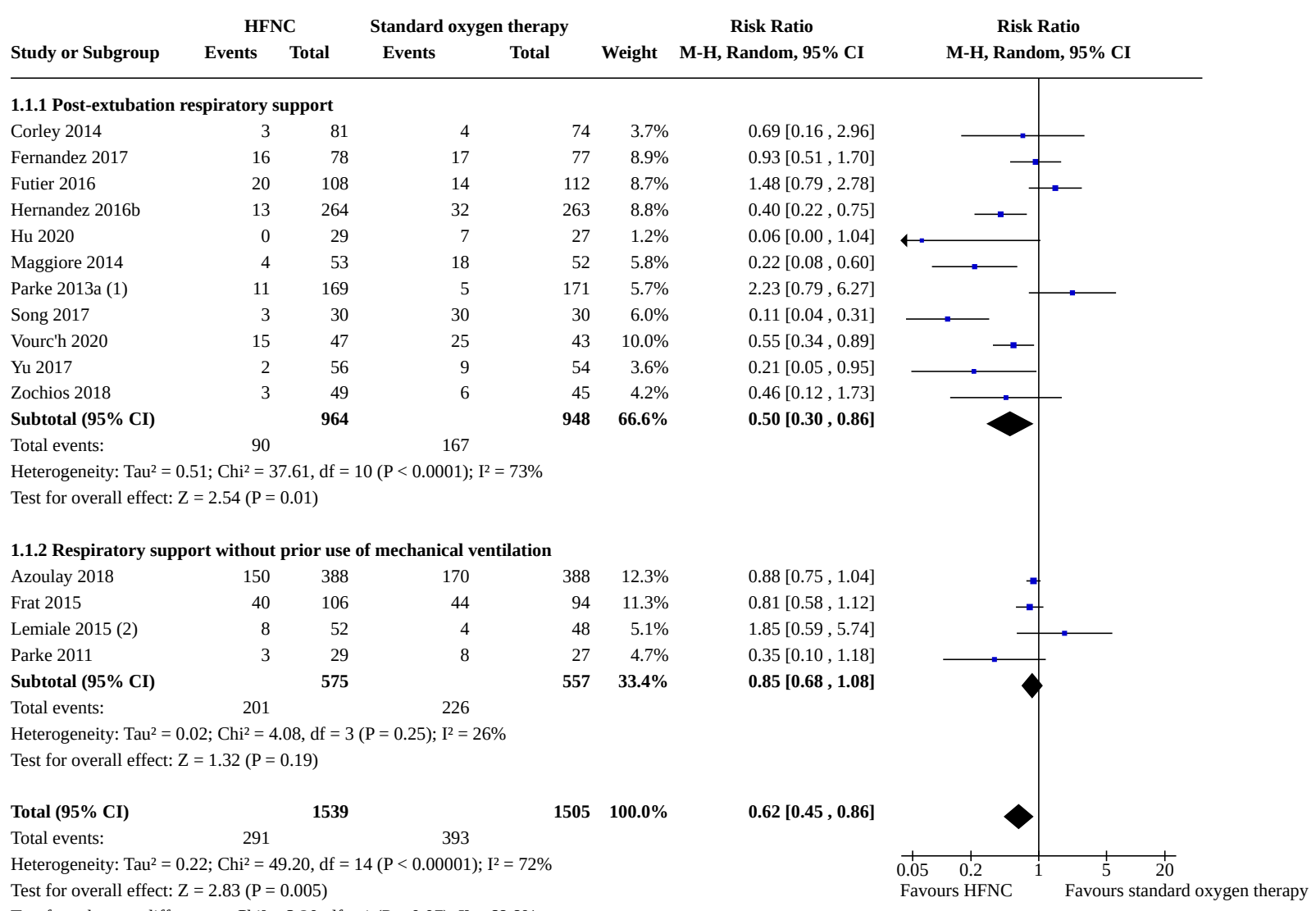

(1) Data only reported at day 2

(2) within 2 hours

Analysis 1.2. Comparison 1: HFNC versus standard oxygen therapy, Outcome 2: In-hospital mortality

\begin{tabular}{|c|c|c|c|c|c|c|c|c|c|}
\hline \multirow[b]{2}{*}{ Study or Subgroup } & \multicolumn{2}{|c|}{ HFNC } & \multicolumn{2}{|c|}{ Standard oxygen therapy } & \multirow[b]{2}{*}{ Weight } & Risk Ratio & \multirow{2}{*}{\multicolumn{2}{|c|}{$\begin{array}{c}\text { Risk Ratio } \\
\text { M-H, Random, 95\% CI }\end{array}$}} & \\
\hline & Events & Total & Events & Total & & M-H, Random, 95\% CI & & & \\
\hline Azoulay 2018 & 160 & 388 & 162 & 388 & $83.4 \%$ & $0.99[0.84,1.17]$ & & & \\
\hline Fernandez 2017 & 12 & 78 & 12 & 77 & $4.3 \%$ & $0.99[0.47,2.06]$ & & & \\
\hline Frat 2015 & 12 & 106 & 18 & 94 & $5.1 \%$ & $0.59[0.30,1.16]$ & & - & \\
\hline Futier 2016 & 2 & 108 & 3 & 112 & $0.7 \%$ & $0.69[0.12,4.06]$ & & & \\
\hline Hernandez 2016b & 10 & 264 & 13 & 263 & $3.6 \%$ & $0.77[0.34,1.72]$ & & - & \\
\hline Hu 2020 & 2 & 29 & 1 & 27 & $0.4 \%$ & $1.86[0.18,19.38]$ & & . & $\longrightarrow$ \\
\hline Maggiore 2014 (1) & 6 & 53 & 5 & 52 & $1.8 \%$ & $1.18[0.38,3.62]$ & & & \\
\hline Parke 2013a & 1 & 169 & 1 & 171 & $0.3 \%$ & $1.01[0.06,16.05]$ & $\longleftarrow$ & & $\longrightarrow$ \\
\hline Vourc'h 2020 & 0 & 47 & 0 & 43 & & Not estimable & & & \\
\hline Yu 2017 & 0 & 56 & 0 & 54 & & Not estimable & & & \\
\hline Zochios 2018 & 1 & 49 & 1 & 45 & $0.3 \%$ & $0.92[0.06,14.25]$ & $\longleftarrow$ & & \\
\hline Total (95\% CI) & & 1347 & & 1326 & $100.0 \%$ & $0.96[0.82,1.11]$ & & & \\
\hline Total events: & 206 & & 216 & & & & & & \\
\hline \multicolumn{7}{|c|}{ Heterogeneity: $\mathrm{Tau}^{2}=0.00 ; \mathrm{Chi}^{2}=2.99, \mathrm{df}=8(\mathrm{P}=0.94) ; \mathrm{I}^{2}=0 \%$} & $\begin{array}{ccc} \\
.1 & 0.2 & 0.5\end{array}$ & 2 & $\begin{array}{ll}5 & 10 \\
5\end{array}$ \\
\hline \multicolumn{7}{|c|}{ Test for overall effect: $\mathrm{Z}=0.57(\mathrm{P}=0.57)$} & Favours HFNC & Favours s & standard oxygen therapy \\
\hline \multicolumn{10}{|c|}{ Test for subgroup differences: Not applicable } \\
\hline
\end{tabular}


Analysis 1.3. Comparison 1: HFNC versus standard oxygen therapy, Outcome 3: Important adverse events

\begin{tabular}{|c|c|c|c|c|c|c|}
\hline \multirow[b]{2}{*}{ Study or Subgroup } & \multicolumn{2}{|c|}{ HFNC } & \multicolumn{2}{|c|}{ Standard oxygen therapy } & \multirow[b]{2}{*}{ Weight } & \multirow{2}{*}{$\begin{array}{c}\text { Risk Ratio } \\
\text { M-H, Random, 95\% CI }\end{array}$} \\
\hline & Events & Total & Events & Total & & \\
\hline \multicolumn{7}{|l|}{ 1.3.1 Pneumonia } \\
\hline Frat 2015 (1) & 19 & 106 & 26 & 94 & $62.2 \%$ & $0.65[0.38,1.09$ \\
\hline Futier 2016 (2) & 10 & 108 & 10 & 112 & $24.3 \%$ & $1.04[0.45,2.39$ \\
\hline Hernandez 2016b (3) & 3 & 264 & 6 & 263 & $9.0 \%$ & $0.50[0.13,1.97$ \\
\hline Yu 2017 (4) & 2 & 56 & 2 & 54 & $4.6 \%$ & $0.96[0.14,6.60$ \\
\hline Subtotal $(95 \% \mathrm{CI})$ & & 534 & & 523 & $100.0 \%$ & $0.72[0.48,1.09$ \\
\hline
\end{tabular}

$\begin{array}{ll}\text { Total events: } & 34 \\ \text { Heterogeneity: } \text { Tau }^{2}=0.00 & \mathrm{Chi}^{2}=1.25, \mathrm{df}=3(\mathrm{P}=0.74) ; \mathrm{I}^{2}=0 \%\end{array}$

Test for overall effect: $\mathrm{Z}=1.55(\mathrm{P}=0.12)$

\begin{tabular}{|c|c|c|}
\hline \multicolumn{3}{|c|}{ 1.3.2 Nasal mucosa or skin trauma } \\
\hline Hernandez 2016b & 0 & 264 \\
\hline Vourc'h 2020 & 4 & 47 \\
\hline Subtotal (95\% CI) & & 311 \\
\hline Total events: & 4 & \\
\hline \multicolumn{3}{|c|}{ Heterogeneity: Not applicable } \\
\hline \multicolumn{3}{|c|}{ Test for overall effect: $\mathrm{Z}=1.18(\mathrm{P}=0.24)$} \\
\hline \multicolumn{3}{|c|}{ Test for subgroup differences: $\mathrm{Chi}^{2}=2.11, \mathrm{df}=$} \\
\hline \multicolumn{3}{|l|}{ Footnotes } \\
\hline \multicolumn{3}{|c|}{ (1) Nosocomial pneumonia } \\
\hline \multicolumn{3}{|c|}{ (2) Pneumonia: not specified } \\
\hline \multicolumn{3}{|c|}{ (3) Ventilator-associated pneumonia } \\
\hline (4) Suspected pneur & & \\
\hline
\end{tabular}

Not estimable $\begin{array}{rrr}43 & 100.0 \% & 3.66[0.43,31.48] \\ \mathbf{3 0 6} & \mathbf{1 0 0 . 0 \%} & \mathbf{3 . 6 6}[\mathbf{0 . 4 3}, \mathbf{3 1 . 4 8}]\end{array}$ $306 \quad 100.0 \%$ $3.66[0.43,31.48]$

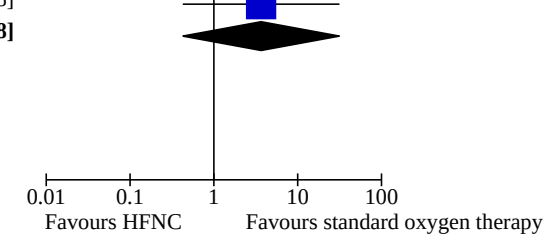

Analysis 1.4. Comparison 1: HFNC versus standard oxygen therapy, Outcome 4: Length of ICU stay (days)

\begin{tabular}{|c|c|c|c|c|c|c|c|c|}
\hline \multirow[b]{2}{*}{ Study or Subgroup } & \multirow[b]{2}{*}{ Mean } & \multirow{2}{*}{$\begin{array}{l}\text { HFNC } \\
\text { SD }\end{array}$} & \multirow[b]{2}{*}{ Total } & \multicolumn{3}{|c|}{ Standard oxygen therapy } & \multirow[b]{2}{*}{ Weight } & \multirow{2}{*}{$\begin{array}{c}\text { Mean Difference } \\
\text { IV, Random, 95\% CI }\end{array}$} \\
\hline & & & & Mean & SD & Total & & \\
\hline Corley 2014 & 1.61 & 1.47 & 81 & 1.61 & 1 & 74 & $14.2 \%$ & $0.00[-0.39,0.39]$ \\
\hline Frat 2015 (1) & 10.7 & 15.8 & 94 & 9.1 & 11.7 & 76 & $0.1 \%$ & $1.60[-2.54,5.74]$ \\
\hline Maggiore 2014 & 11.7 & 10.2 & 53 & 10.4 & 8.5 & 52 & $0.2 \%$ & $1.30[-2.29,4.89]$ \\
\hline Parke 2013a & 1.39 & 0.95 & 169 & 1.2 & 1 & 171 & $51.1 \%$ & $0.19[-0.02,0.40]$ \\
\hline Vourc'h 2020 & 3.3 & 2.4 & 47 & 3.1 & 1.6 & 43 & $3.1 \%$ & $0.20[-0.64,1.04]$ \\
\hline Yu 2017 & 3.72 & 0.56 & 56 & 3.64 & 0.83 & 54 & $31.2 \%$ & $0.08[-0.19,0.35]$ \\
\hline Total $(95 \%$ CI $)$ & & & 500 & & & 470 & $100.0 \%$ & $0.13[-0.02,0.28]$ \\
\hline
\end{tabular}

Heterogeneity: $\mathrm{Tau}^{2}=0.00 ; \mathrm{Chi}^{2}=1.80, \mathrm{df}=5(\mathrm{P}=0.88) ; \mathrm{I}^{2}=0 \%$

$470 \quad 100.0 \%$

$0.13[-0.02,0.28]$

Test for overall effect: $\mathrm{Z}=1.75(\mathrm{P}=0.08)$

Test for subgroup differences: Not applicable

Mean Difference

IV, Random, $95 \%$ CI

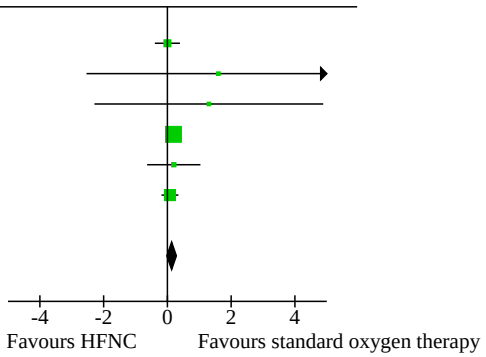

Footnotes

(1) survivors at 90 days (standard oxygen therapy) 


\section{Analysis 1.5. Comparison 1: HFNC versus standard oxygen therapy,} Outcome 5: Short-term respiratory effects: $\mathrm{PaO}_{2} / \mathrm{FiO}_{2}(\mathrm{mmHg})$

\begin{tabular}{|c|c|c|c|c|c|}
\hline \multirow[b]{2}{*}{ Study or Subgroup } & \multicolumn{3}{|c|}{ HFNC } & \multicolumn{2}{|c|}{ Standard oxy } \\
\hline & Mean & SD & Total & Mean & \\
\hline Corley 2014 (1) & 281.4 & 85.6659 & 81 & 253.3 & \\
\hline Frat $2015(2)$ & 130 & 60 & 106 & 161 & \\
\hline Maggiore 2014 (3) & 287.5 & 74.3 & 53 & 247.4 & \\
\hline Parke 2011 (4) & 177.8 & 50.2 & 28 & 181.7 & \\
\hline Vourc'h 2020 & 129.9 & 54 & 47 & 106.9 & \\
\hline \multicolumn{3}{|l|}{ Total (95\% CI) } & 315 & & \\
\hline \multicolumn{6}{|c|}{ Heterogeneity: Tau $^{2}=822.79 ; \mathrm{Chi}^{2}=24.03, \mathrm{df}=4(\mathrm{P}<0.0001) ; \mathrm{I}^{2}=83 \%$} \\
\hline \multicolumn{6}{|c|}{ Test for overall effect: $\mathrm{Z}=0.73(\mathrm{P}=0.46)$} \\
\hline \multicolumn{6}{|c|}{ Test for subgroup differences: Not applicable } \\
\hline \multicolumn{6}{|l|}{ Footnotes } \\
\hline \multicolumn{6}{|l|}{ (1) first 24 hours } \\
\hline \multicolumn{6}{|c|}{ (2) HFNC vs standard oxygen therapy (at 6 hours) } \\
\hline \multicolumn{6}{|c|}{ (3) At 24 hours } \\
\hline (4) At four hours & & & & & \\
\hline
\end{tabular}

Mean Difference Mean Difference

IV, Random, 95\% CI IV, Random, 95\% CI

Analysis 1.6. Comparison 1: HFNC versus standard oxygen therapy, Outcome 6: Comfort

\begin{tabular}{|c|c|c|c|c|c|c|c|c|c|}
\hline & & HFNC & & Stand & oxyg & erapy & & Mean Difference & Mean Difference \\
\hline Study or Subgroup & Mean & SD & Total & Mean & SD & Total & Weight & IV, Random, 95\% CI & IV, Random, 95\% CI \\
\hline
\end{tabular}

\begin{tabular}{lllrrrrrr}
\hline 1.6.1 Short-term effect & & & & & & & & \\
Frat 2015 (1) & 7.1 & 2.6 & 106 & 6 & 2.9 & 94 & $26.7 \%$ & $1.10[0.33,1.87]$ \\
Maggiore 2014 (2) & 4.8 & 3.4 & 53 & 4.4 & 3.5 & 52 & $19.5 \%$ & $0.40[-0.92,1.72]$ \\
Parke 2013a (3) & 7.5 & 2.6 & 169 & 8.1 & 2.3 & 171 & $29.7 \%$ & $-0.60[-1.12,-0.08]$ \\
Rittayamai 2014 (4) & 8.6 & 0.9 & 9 & 8.1 & 1.1 & 8 & $24.1 \%$ & $0.50[-0.46,1.46]$ \\
Subtotal (95\% CI) & & & $\mathbf{3 3 7}$ & & & $\mathbf{3 2 5}$ & $\mathbf{1 0 0 . 0 \%}$ & $\mathbf{0 . 3 1}[-\mathbf{0 . 6 0}, \mathbf{1 . 2 2}]$
\end{tabular}

Heterogeneity: $\mathrm{Tau}^{2}=0.65 ; \mathrm{Chi}^{2}=14.26, \mathrm{df}=3(\mathrm{P}=0.003) ; \mathrm{I}^{2}=79 \%$

$\begin{array}{rrr}6659 & 74 & 19.7 \% \\ 77 & 94 & 21.6 \%\end{array}$

$28.10[1.10,55.10]$

$-31.00[-50.31,-11.69]$

$40.10[10.43,69.77]$

$-3.90[-31.96,24.16]$

$23.00[-1.26,47.26]$

$\begin{array}{lll}50.3 & 22 & 19.4 \% \\ 62.6 & 43 & 20.4 \%\end{array}$

$10.34[-17.31,38.00]$

$285100.0 \%$

Test for overall effect: $\mathrm{Z}=0.68(\mathrm{P}=0.50)$

$\begin{array}{lrrrrrrrr}\text { 1.6.2 Long-term effect } & & & & & & & & \\ \text { Maggiore 2014 (5) } & 8.5 & 1.9 & 53 & 6.4 & 3.4 & 52 & 48.7 \% & 2.10[1.04,3.16] \\ \text { Parke 2013a (6) } & 6.94 & 2.5 & 169 & 7.78 & 1.9 & 171 & 51.3 \% & -0.84[-1.31,-0.37] \\ \text { Subtotal (95\% CI) } & & & \mathbf{2 2 2} & & & \mathbf{2 2 3} & \mathbf{1 0 0 . 0 \%} & \mathbf{0 . 5 9}[-\mathbf{2 . 2 9}, \mathbf{3 . 4 7}]\end{array}$

Heterogeneity: $\mathrm{Tau}^{2}=4.15 ; \mathrm{Chi}^{2}=24.80, \mathrm{df}=1(\mathrm{P}<0.00001) ; \mathrm{I}^{2}=96 \%$

Test for overall effect: $\mathrm{Z}=0.40(\mathrm{P}=0.69)$

Footnotes

(1) At 1 hour. To allow calculation of the mean difference, we converted the $0-100 \mathrm{~mm}$ scale to a $0-10$ scale by dividing the mean and SD by 10 .

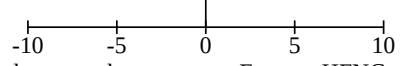

(2) at 1 hour

(3) at 4 hours

(4) at 30 minutes

(5) At 48 hours

(6) At day 2 
Analysis 1.7. Comparison 1: HFNC versus standard oxygen therapy, Outcome 7: Long-term respiratory effects: $\mathrm{PaO}_{2} / \mathrm{FiO}_{2}(\mathrm{mmHg})$

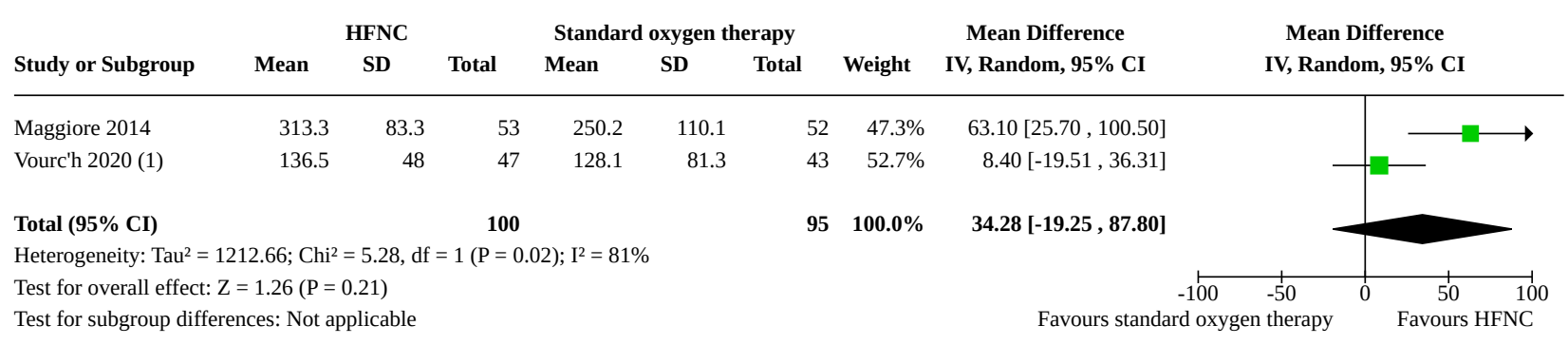

Footnotes

(1) At 48 hours

Analysis 1.8. Comparison 1: HFNC versus standard oxygen therapy, Outcome 8: Short-term and long-term respiratory effects: $\mathrm{PaO}_{2}(\mathrm{mmHg})$

\begin{tabular}{|c|c|c|c|c|c|c|c|c|}
\hline \multirow[b]{2}{*}{ Study or Subgroup } & \multirow[b]{2}{*}{ Mean } & \multicolumn{2}{|l|}{ HFNC } & \multicolumn{3}{|c|}{ Standard oxygen therapy } & \multirow[b]{2}{*}{ Weight } & \multirow{2}{*}{$\begin{array}{c}\text { Mean Difference } \\
\text { IV, Random, 95\% CI }\end{array}$} \\
\hline & & SD & Total & Mean & SD & Total & & \\
\hline \multicolumn{9}{|c|}{ 1.8.1 Short-term effects } \\
\hline Frat 2015 (1) & 90 & 35 & 106 & 93 & 36 & 94 & $22.6 \%$ & $-3.00[-12.87,6.87]$ \\
\hline Maggiore 2014 (2) & 95 & 28 & 53 & 84.6 & 22.6 & 52 & $23.0 \%$ & $10.40[0.68,20.12]$ \\
\hline Parke 2011 (3) & 80.2 & 11.8 & 28 & 79.7 & 25.6 & 22 & $18.6 \%$ & $0.50[-11.06,12.06]$ \\
\hline Song 2017 & 83.2 & 10.5 & 30 & 74.5 & 13.1 & 30 & $35.7 \%$ & $8.70[2.69,14.71]$ \\
\hline Subtotal (95\% CI) & & & 217 & & & 198 & $100.0 \%$ & $4.92[-1.24,11.07]$ \\
\hline
\end{tabular}

Heterogeneity: $\mathrm{Tau}^{2}=18.19 ; \mathrm{Chi}^{2}=5.62, \mathrm{df}=3(\mathrm{P}=0.13) ; \mathrm{I}^{2}=47 \%$

Test for overall effect: $\mathrm{Z}=1.57(\mathrm{P}=0.12)$

1.8.2 Long-term effects

$\begin{array}{lrrrrrrrr}\text { Hu 2020 (4) } & 102.4 & 26.4 & 29 & 86.6 & 26.4 & 27 & 11.8 \% & 15.80[1.96,29.64] \\ \text { Maggiore 2014 (5) } & 97.2 & 29.2 & 536 & 85.4 & 16.3 & 52 & 88.2 \% & 11.80[6.73,16.87] \\ \text { Subtotal (95\% CI) } & & & \mathbf{5 6 5} & & & \mathbf{7 9} & \mathbf{1 0 0 . 0 \%} & \mathbf{1 2 . 2 7}[\mathbf{7 . 5 1 , \mathbf { 1 7 . 0 4 } ]}\end{array}$

Heterogeneity: $\mathrm{Tau}^{2}=0.00 ; \mathrm{Chi}^{2}=0.28, \mathrm{df}=1(\mathrm{P}=0.59) ; \mathrm{I}^{2}=0 \%$

Test for overall effect: $\mathrm{Z}=5.05(\mathrm{P}<0.00001)$

Test for subgroup differences: $\mathrm{Chi}^{2}=3.43, \mathrm{df}=1(\mathrm{P}=0.06), \mathrm{I}^{2}=70.9 \%$

Mean Difference IV, Random, 95\% CI

Footnotes

(1) HFNC vs standard oxygen (at 6 hours)

(2) At 24 hours

(3) At four hours

(4) At 48 hours

(5) At 36 hours 
Analysis 1.9. Comparison 1: HFNC versus standard oxygen therapy, Outcome 9: Short-term and long-term respiratory effects: $\mathrm{SpO}_{2}$ (\%)

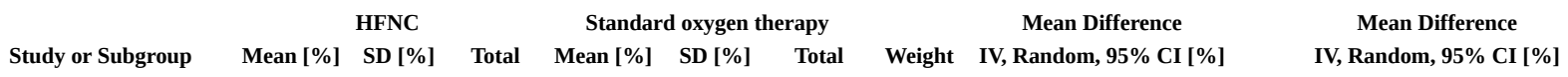

\begin{tabular}{lrrrrrrrr}
\hline 1.9.1 Short-term effects & & & & & & & & \\
Maggiore 2014 (1) & 97 & 2 & 53 & 95 & 2 & 52 & $22.0 \%$ & $2.00[1.23,2.77]$ \\
Parke 2011 (2) & 95 & 2 & 28 & 95.4 & 2.9 & 22 & $17.4 \%$ & $-0.40[-1.82,1.02]$ \\
Parke 2013a (3) & 96.6 & 2.1 & 169 & 96.9 & 1.9 & 171 & $23.8 \%$ & $-0.30[-0.73,0.13]$ \\
Rittayamai 2014 (4) & 99.11 & 1.45 & 9 & 97.38 & 2.34 & 8 & $14.2 \%$ & $1.73[-0.15,3.61]$ \\
Song 2017 & 98 & 1.3 & 30 & 96.9 & 1.4 & 30 & $22.5 \%$ & $1.10[0.42,1.78]$ \\
Subtotal (95\% CI) & & & $\mathbf{2 8 9}$ & & & $\mathbf{2 8 3}$ & $\mathbf{1 0 0 . 0 \%}$ & $\mathbf{0 . 7 9}[-\mathbf{0 . 2 9}, \mathbf{1 . 8 8}]$
\end{tabular}

Heterogeneity: $\mathrm{Tau}^{2}=1.24 ; \mathrm{Chi}^{2}=34.30, \mathrm{df}=4(\mathrm{P}<0.00001) ; \mathrm{I}^{2}=88 \%$

Test for overall effect: $\mathrm{Z}=1.43(\mathrm{P}=0.15)$

1.9.2 Long-term effects

Maggiore 2014 (5)

Parke 2013a (6)

$\begin{array}{rrr}97 & 2 & 53 \\ 95.6 & 2.6 & 169\end{array}$

$53 \quad 95$

$95 \quad 3 \quad 52 \quad 45.0 \%$

$171 \quad 55.0 \%$

Subtotal $(95 \%$ CI)

222

$223 \quad 100.0 \%$

$0.70[0.15,1.25]$

$1.28[0.02,2.55]$

Heterogeneity: $\mathrm{Tau}^{2}=0.68 ; \mathrm{Chi}^{2}=5.15, \mathrm{df}=1(\mathrm{P}=0.02) ; \mathrm{I}^{2}=81 \%$

Test for overall effect: $\mathrm{Z}=1.99(\mathrm{P}=0.05)$

Test for subgroup differences: $\mathrm{Chi}^{2}=0.33, \mathrm{df}=1(\mathrm{P}=0.56), \mathrm{I}^{2}=0 \%$

IV, Random, 95\% CI [\%]

Footnotes

(1) At 24 hours

(2) At four hours

(3) At day 1

(4) At 30 minutes

(5) At 48 hours

(6) At day 2

\section{Analysis 1.10. Comparison 1: HFNC versus standard oxygen therapy, Outcome 10: Short-term respiratory effects: $\mathrm{PaCO}_{2}(\mathrm{mmHg})$}

\begin{tabular}{|c|c|c|c|c|c|c|c|c|c|c|}
\hline \multirow[b]{2}{*}{ Study or Subgroup } & \multirow[b]{2}{*}{ Mean } & \multicolumn{2}{|l|}{ HFNC } & \multicolumn{3}{|c|}{ Standard oxygen therapy } & \multirow[b]{2}{*}{ Weight } & \multirow{2}{*}{$\begin{array}{c}\text { Mean Difference } \\
\text { IV, Random, } 95 \% \text { CI }\end{array}$} & \multirow{2}{*}{\multicolumn{2}{|c|}{$\begin{array}{c}\text { Mean Difference } \\
\text { IV, Random, 95\% CI }\end{array}$}} \\
\hline & & SD & Total & Mean & SD & Total & & & & \\
\hline Frat 2015 (1) & 36 & 7 & 106 & 36 & 6 & 94 & $27.0 \%$ & $0.00[-1.80,1.80]$ & & \\
\hline Maggiore 2014 (2) & 32.3 & 7.1 & 53 & 36.2 & 11.2 & 52 & $9.5 \%$ & $-3.90[-7.49,-0.31]$ & & \\
\hline Parke 2011 & 40.6 & 6 & 28 & 39.8 & 6.3 & 22 & $10.2 \%$ & $0.80[-2.65,4.25]$ & & \\
\hline Parke 2013a (3) & 38.2 & 4.95 & 169 & 39.7 & 4 & 171 & $48.5 \%$ & $-1.50[-2.46,-0.54]$ & $\longrightarrow$ & \\
\hline Song 2017 & 41.4 & 6.5 & 30 & 42.2 & 13.1 & 30 & $4.8 \%$ & $-0.80[-6.03,4.43]$ & $\longleftarrow$ & \\
\hline Total (95\% CI) & & & 386 & & & 369 & $100.0 \%$ & $-1.05[-2.24,0.13]$ & & \\
\hline \multicolumn{11}{|c|}{ Heterogeneity: $\mathrm{Tau}^{2}=0.52 ; \mathrm{Chi}^{2}=5.56, \mathrm{df}=4(\mathrm{P}=0.23) ; \mathrm{I}^{2}=28 \%$} \\
\hline \multicolumn{9}{|c|}{ Test for overall effect: $\mathrm{Z}=1.74(\mathrm{P}=0.08)$} & $\begin{array}{rr} & -1 \\
& -1\end{array}$ & $\begin{array}{lll}1 & 1 & 1 \\
0 & 1 & 2\end{array}$ \\
\hline \multicolumn{9}{|c|}{ Test for subgroup differences: Not applicable } & Favours HFNC & Favours \\
\hline
\end{tabular}

Footnotes

(1) HFNC versus standard oxygen

(2) At 3 hours

(3) At day 1 


\section{Analysis 1.11. Comparison 1: HFNC versus standard oxygen therapy,}

Outcome 11: Short-term and long-term respiratory rate (breaths/min)

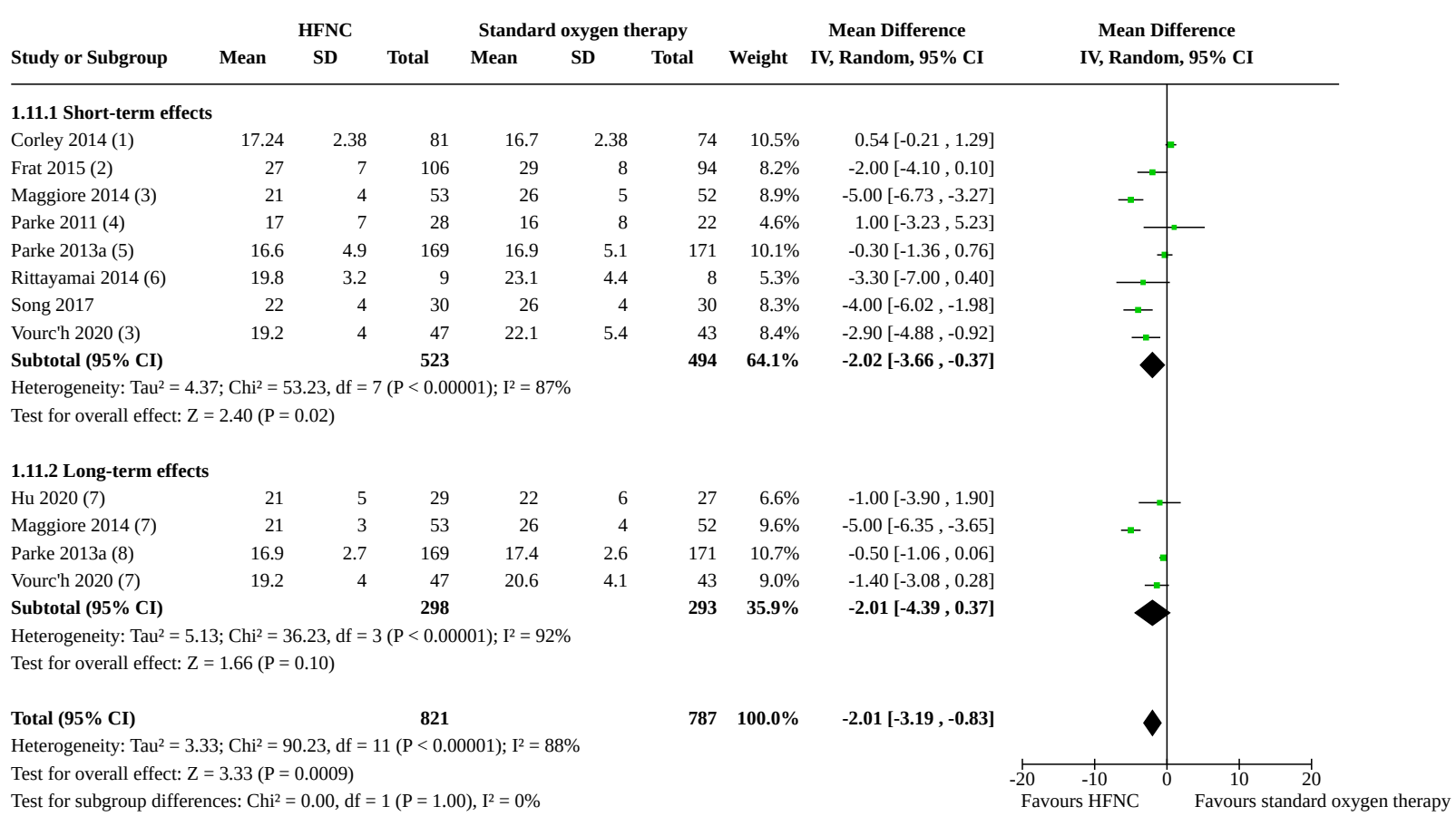

Footnotes

(1) first 24 hours

(2) HFNC versus standard oxygen (at 6 hours)

(3) At 24 hours

(4) at four hours

(5) At day 1

(6) at 30 minutes

(7) At 48 hours

(8) At day 2

Analysis 1.12. Comparison 1: HFNC versus standard oxygen therapy, Outcome 12: Length of hospital stay (days)

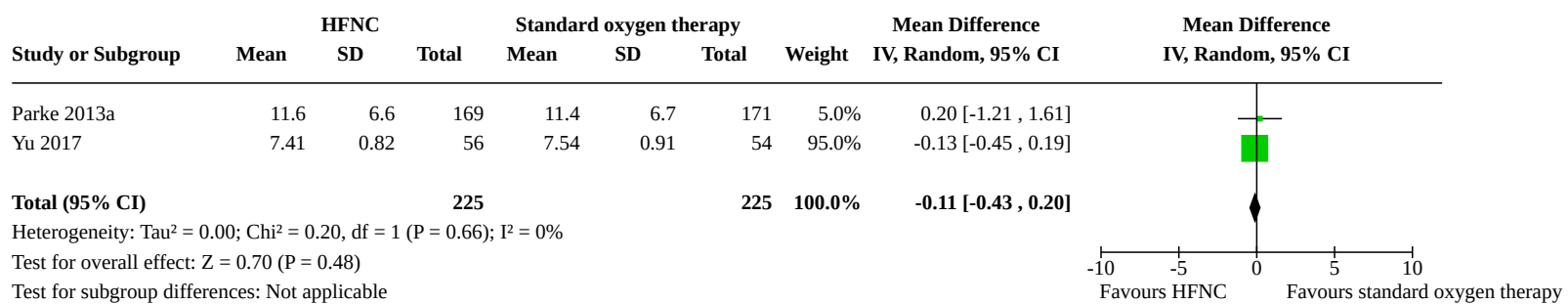




\section{Analysis 1.13. Comparison 1: HFNC versus standard oxygen therapy, Outcome 13: Refusal to continue with treatment}

\begin{tabular}{|c|c|c|c|c|c|c|c|c|}
\hline \multirow[b]{2}{*}{ Study or Subgroup } & \multicolumn{2}{|c|}{ HFNC } & \multicolumn{2}{|c|}{ Standard oxygen therapy } & \multirow[b]{2}{*}{ Weight } & \multirow{2}{*}{$\begin{array}{c}\text { Risk Ratio } \\
\text { M-H, Random, 95\% CI }\end{array}$} & \multirow{2}{*}{\multicolumn{2}{|c|}{$\begin{array}{c}\text { Risk Ratio } \\
\text { M-H, Random, 95\% CI }\end{array}$}} \\
\hline & Events & Total & Events & Total & & & & \\
\hline Futier 2016 & 8 & 108 & 0 & 112 & $49.2 \%$ & $17.62[1.03,301.65]$ & & \\
\hline Parke 2013a & 20 & 171 & 0 & 169 & $50.8 \%$ & $40.52[2.47,664.65]$ & & \\
\hline Total (95\% CI) & & 279 & & 281 & $100.0 \%$ & $26.89[3.67,197.32]$ & & \\
\hline Total events: & 28 & & 0 & & & & & \\
\hline \multirow{2}{*}{\multicolumn{5}{|c|}{ Heterogeneity: $\mathrm{Tau}^{2}=0.00 ; \mathrm{Chi}^{2}=0.17, \mathrm{df}=1(\mathrm{P}=0.68) ; \mathrm{I}^{2}=0 \%$}} & & & $0.01 \quad 0.1$ & 10 \\
\hline & & & & & & & Favours HFNC & Favours s \\
\hline
\end{tabular}

\section{Comparison 2. HFNC versus NIPPV or NIV}

\begin{tabular}{|c|c|c|c|c|}
\hline Outcome or subgroup title & No. of studies & $\begin{array}{l}\text { No. of partici- } \\
\text { pants }\end{array}$ & Statistical method & Effect size \\
\hline $\begin{array}{l}2.1 \text { Treatment failure (escalation of } \\
\text { respiratory support to NIV, NIPPV or } \\
\text { invasive ventilation) }\end{array}$ & 5 & 1758 & $\begin{array}{l}\text { Risk Ratio (M-H, Random, } \\
95 \% \mathrm{Cl})\end{array}$ & $0.98[0.78,1.22]$ \\
\hline $\begin{array}{l}\text { 2.1.1 Post-extubation respiratory sup- } \\
\text { port }\end{array}$ & 3 & 1472 & $\begin{array}{l}\text { Risk Ratio (M-H, Random, } \\
95 \% \mathrm{Cl})\end{array}$ & $1.12[0.89,1.41]$ \\
\hline $\begin{array}{l}\text { 2.1.2 Respiratory support without pri- } \\
\text { or use of mechanical ventilation }\end{array}$ & 2 & 286 & $\begin{array}{l}\text { Risk Ratio (M-H, Random, } \\
95 \% \mathrm{Cl})\end{array}$ & $0.77[0.58,1.03]$ \\
\hline 2.2 In-hospital mortality & 5 & 1758 & $\begin{array}{l}\text { Risk Ratio (M-H, Random, } \\
95 \% \mathrm{Cl})\end{array}$ & $0.92[0.64,1.31]$ \\
\hline $\begin{array}{l}2.3 \text { Important adverse events: pneu- } \\
\text { monia }\end{array}$ & 3 & 1750 & $\begin{array}{l}\text { Risk Ratio (M-H, Random, } \\
95 \% \mathrm{Cl})\end{array}$ & $0.51[0.17,1.52]$ \\
\hline $\begin{array}{l}\text { 2.4 Short-term respiratory effects: } \\
\mathrm{PaO}_{2} / \mathrm{FiO}_{2}(\mathrm{mmHg})\end{array}$ & 3 & 1086 & $\begin{array}{l}\text { Mean Difference (IV, Random, } \\
95 \% \mathrm{CI} \text { ) }\end{array}$ & $\begin{array}{l}-58.10[-71.68 \\
-44.51]\end{array}$ \\
\hline 2.5 Length of ICU stay (days) & 2 & 246 & $\begin{array}{l}\text { Mean Difference (IV, Random, } \\
95 \% \mathrm{CI} \text { ) }\end{array}$ & $-0.72[-2.85,1.42]$ \\
\hline $\begin{array}{l}2.6 \text { Short-term comfort (continuous } \\
\text { data) }\end{array}$ & 2 & 258 & $\begin{array}{l}\text { Mean Difference (IV, Random, } \\
95 \% \mathrm{CI} \text { ) }\end{array}$ & $1.33[0.74,1.92]$ \\
\hline $\begin{array}{l}\text { 2.7 Duration of respiratory support } \\
\text { (hours) }\end{array}$ & 2 & 210 & $\begin{array}{l}\text { Mean Difference (IV, Random, } \\
95 \% \mathrm{CI} \text { ) }\end{array}$ & $\begin{array}{l}-6.12[-54.61, \\
42.37]\end{array}$ \\
\hline $\begin{array}{l}2.8 \text { Long-term respiratory effects: } \\
\mathrm{PaO}_{2} / \mathrm{FiO}_{2}(\mathrm{mmHg})\end{array}$ & 2 & 344 & $\begin{array}{l}\text { Mean Difference (IV, Random, } \\
95 \% \mathrm{CI} \text { ) }\end{array}$ & $\begin{array}{l}-31.67[-49.37 \\
-13.97]\end{array}$ \\
\hline $\begin{array}{l}2.9 \text { Short-term respiratory effects: } \\
\mathrm{PaO}_{2}(\mathrm{mmHg})\end{array}$ & 2 & 384 & $\begin{array}{l}\text { Mean Difference (IV, Random, } \\
95 \% \mathrm{CI})\end{array}$ & $\begin{array}{l}-9.57[-30.25 \\
11.11]\end{array}$ \\
\hline $\begin{array}{l}\text { 2.10 Short-term and long-term respi- } \\
\text { ratory effects: } \mathrm{PaCO}_{2}(\mathrm{mmHg})\end{array}$ & 4 & & $\begin{array}{l}\text { Mean Difference (IV, Random, } \\
95 \% \mathrm{CI} \text { ) }\end{array}$ & Subtotals only \\
\hline
\end{tabular}




\begin{tabular}{|c|c|c|c|c|}
\hline Outcome or subgroup title & No. of studies & $\begin{array}{l}\text { No. of partici- } \\
\text { pants }\end{array}$ & Statistical method & Effect size \\
\hline 2.10.1 Short-term effects & 4 & 1254 & $\begin{array}{l}\text { Mean Difference (IV, Random, } \\
95 \% \mathrm{CI} \text { ) }\end{array}$ & $-0.46[-2.08,1.16]$ \\
\hline 2.10.2 Long-term effects & 2 & 208 & $\begin{array}{l}\text { Mean Difference (IV, Random, } \\
95 \% \mathrm{CI} \text { ) }\end{array}$ & $-1.80[-5.57,1.98]$ \\
\hline $\begin{array}{l}2.11 \text { Short-term respiratory effects: } \\
\text { breaths/min }\end{array}$ & 4 & 1090 & $\begin{array}{l}\text { Mean Difference (IV, Random, } \\
95 \% \mathrm{CI} \text { ) }\end{array}$ & $-1.06[-1.80,-0.32]$ \\
\hline 2.12 Dyspnoea (any improvement) & 2 & 1023 & $\begin{array}{l}\text { Risk Ratio (M-H, Random, } \\
95 \% \mathrm{Cl} \text { ) }\end{array}$ & $1.05[0.74,1.48]$ \\
\hline
\end{tabular}

\section{Analysis 2.1. Comparison 2: HFNC versus NIPPV or NIV, Outcome 1: Treatment failure (escalation of respiratory support to NIV, NIPPV or invasive ventilation)}

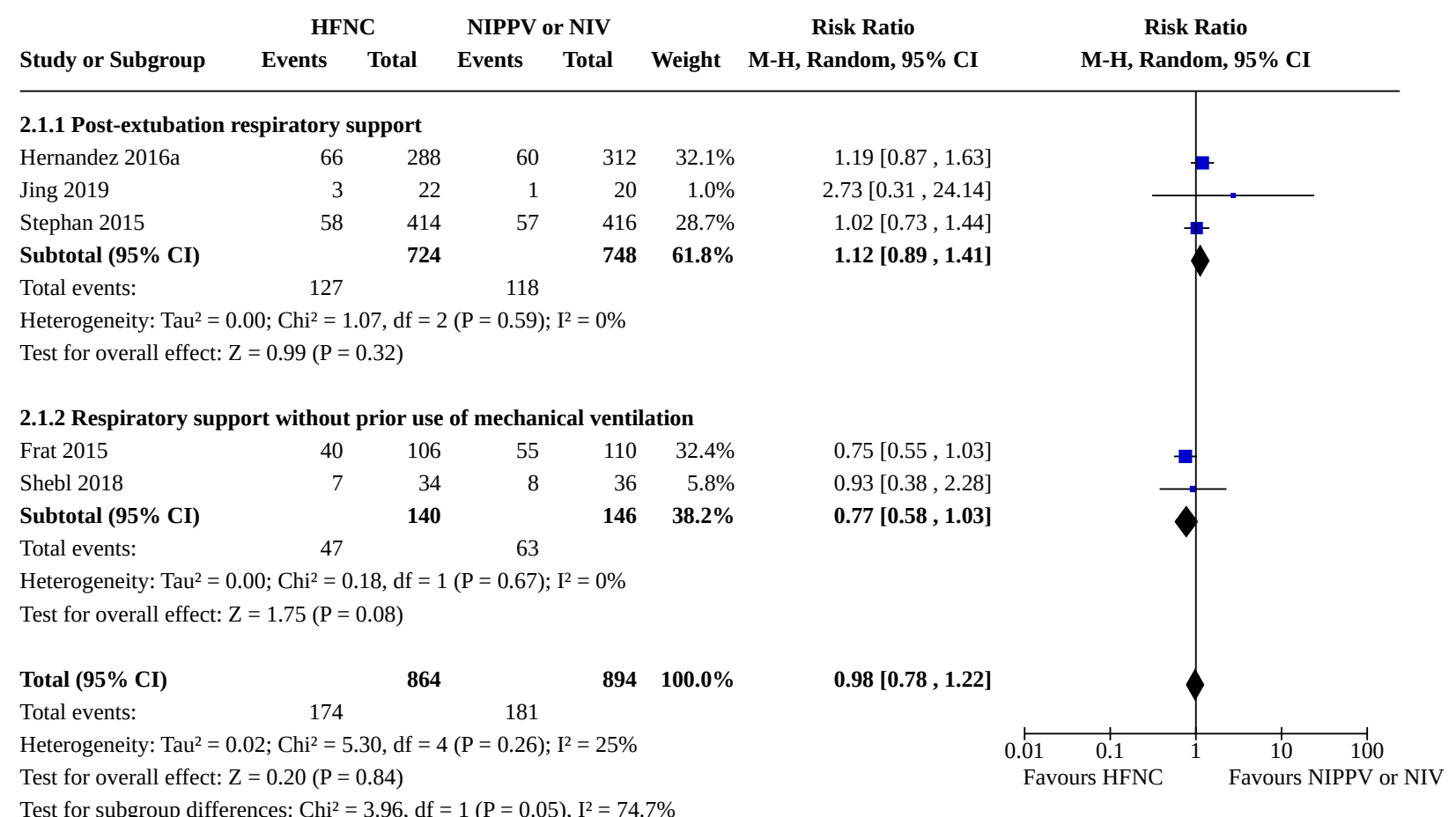


Analysis 2.2. Comparison 2: HFNC versus NIPPV or NIV, Outcome 2: In-hospital mortality

\begin{tabular}{|c|c|c|c|c|c|c|c|}
\hline \multirow[b]{2}{*}{ Study or Subgroup } & \multicolumn{2}{|c|}{ HFNC } & \multicolumn{2}{|c|}{ NIPPV or NIV } & \multirow[b]{2}{*}{ Weight } & \multirow{2}{*}{$\begin{array}{c}\text { Risk Ratio } \\
\text { M-H, Random, 95\% CI }\end{array}$} & \multirow{2}{*}{$\begin{array}{c}\text { Risk Ratio } \\
\text { M-H, Random, 95\% CI }\end{array}$} \\
\hline & Events & Total & Events & Total & & & \\
\hline Frat 2015 & 12 & 106 & 27 & 110 & $19.2 \%$ & $0.46[0.25,0.86]$ & $\rightarrow-$ \\
\hline Hernandez 2016a (1) & 59 & 288 & 56 & 312 & $33.7 \%$ & $1.14[0.82,1.59]$ & \\
\hline Jing 2019 & 5 & 22 & 5 & 20 & $8.8 \%$ & $0.91[0.31,2.68]$ & \\
\hline Shebl 2018 & 9 & 34 & 11 & 36 & $15.4 \%$ & $0.87[0.41,1.83]$ & \\
\hline Stephan 2015 & 28 & 414 & 23 & 416 & $22.9 \%$ & $1.22[0.72,2.09]$ & \\
\hline Total (95\% CI) & & 864 & & 894 & $100.0 \%$ & $0.92[0.64,1.31]$ & \\
\hline Total events: & 113 & & 122 & & & & \\
\hline \multicolumn{5}{|c|}{ Heterogeneity: $\mathrm{Tau}^{2}=0.07 ; \mathrm{Chi}^{2}=7.19, \mathrm{df}=4(\mathrm{P}=0.13) ; \mathrm{I}^{2}=44 \%$} & & 0.01 & 0.1 \\
\hline Test for overall effect: & $=0.49(\mathrm{P}=$ & $0.63)$ & & & & & urs HFNC \\
\hline
\end{tabular}

Test for subgroup differences: Not applicable

Footnotes

(1) We have assumed data reported by study authors is per-protocol and we have accounted for 2 lost participants in each group

Analysis 2.3. Comparison 2: HFNC versus NIPPV or NIV, Outcome 3: Important adverse events: pneumonia

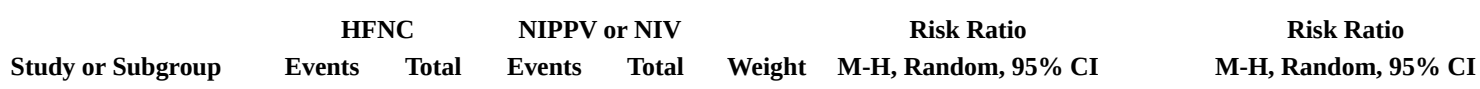

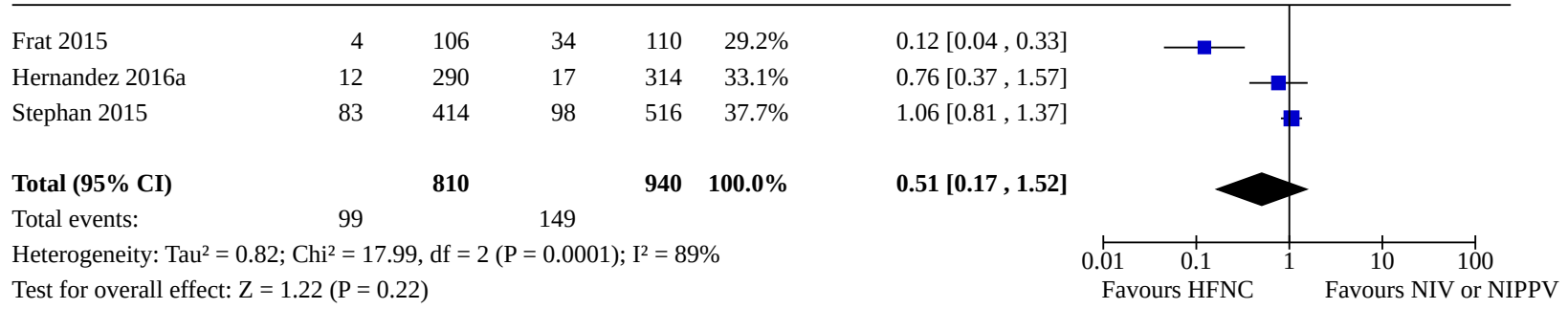

Test for subgroup differences: Not applicable

Analysis 2.4. Comparison 2: HFNC versus NIPPV or NIV, Outcome 4: Short-term respiratory effects: $\mathrm{PaO}_{2} / \mathrm{FiO}_{2}$ ( $\mathrm{mmHg}$ )

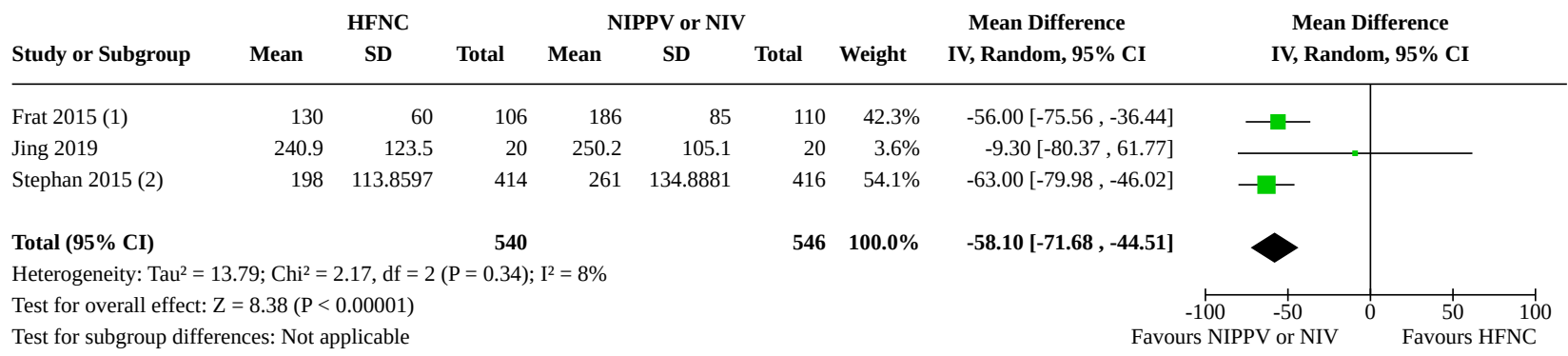

Footnotes

(1) At 6 hours

(2) At 6 to 12 hours 
Analysis 2.5. Comparison 2: HFNC versus NIPPV or NIV, Outcome 5: Length of ICU stay (days)

\begin{tabular}{|c|c|c|c|c|c|c|c|c|c|}
\hline & & HFNC & & & NIV & & & Mean Difference & Mean Difference \\
\hline Study or Subgroup & Mean & SD & Total & Mean & SD & Total & Weight & IV, Random, 95\% CI & IV, Random, 95\% CI \\
\hline
\end{tabular}

$\begin{array}{lllllllll}\text { Frat } 2015(1) & 10.7 & 15.8 & 94 & 11 & 11.6 & 110 & 30.6 \% & -0.30[-4.16,3.56]\end{array}$

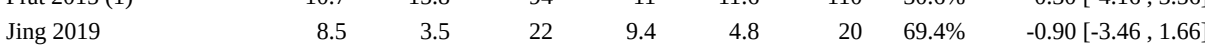

Total (95\% CI)

116

$130 \quad \mathbf{1 0 0 . 0} \%$

$-0.72[-2.85,1.42]$

Heterogeneity: Tau ${ }^{2}=0.00 ; \mathrm{Chi}^{2}=0.06, \mathrm{df}=1(\mathrm{P}=0.80) ; \mathrm{I}^{2}=0 \%$

Test for overall effect: $\mathrm{Z}=0.66(\mathrm{P}=0.51)$

Test for subgroup differences: Not applicable

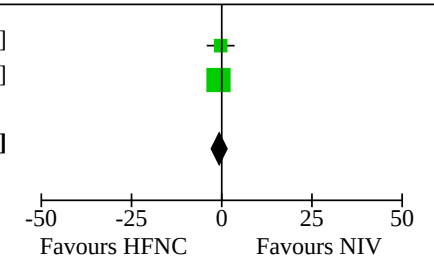

Footnotes

(1) survivors at 90 days (standard oxygen therapy)

Analysis 2.6. Comparison 2: HFNC versus NIPPV or NIV, Outcome 6: Short-term comfort (continuous data)

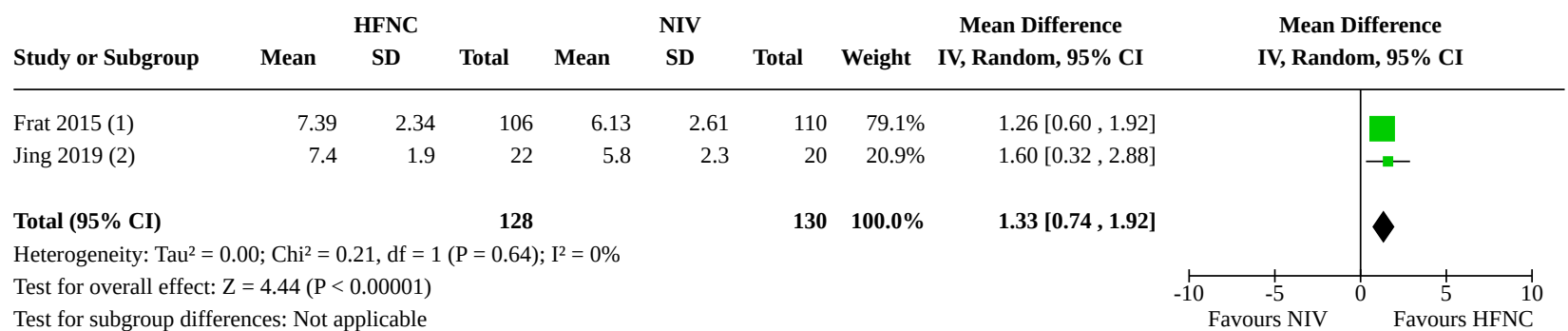

Footnotes

(1) At 1 hour. To allow calculation of the mean difference, we converted the 0-100 mm scale (discomfort higher) to a 1-10 scale (comfort higher). For the mean, we inverter

(2) We inverted these data reported on a 1-10 scale with discomfort being greater. For the mean, we subtracted 1 and then took this number from 10. For the SD we made n

Analysis 2.7. Comparison 2: HFNC versus NIPPV or NIV, Outcome 7: Duration of respiratory support (hours)

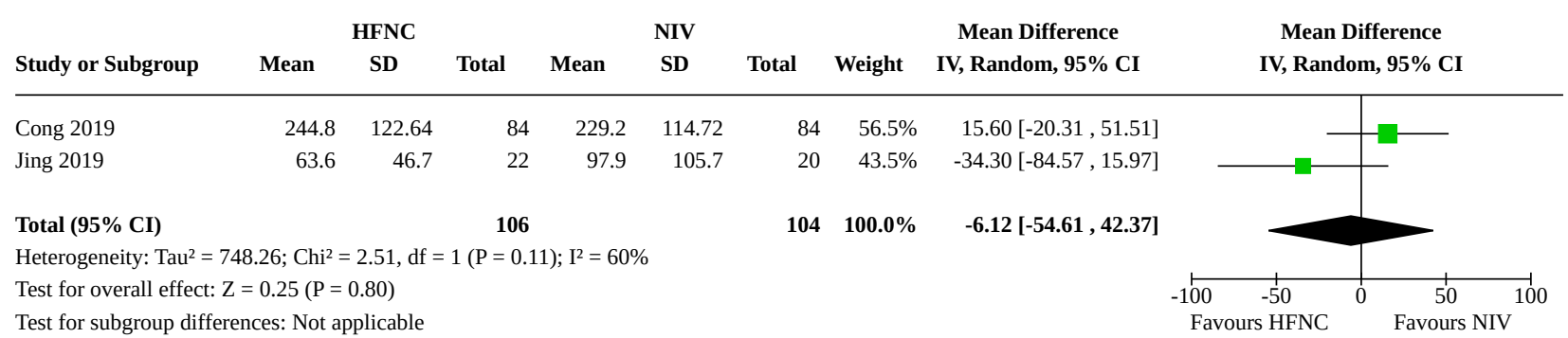


Analysis 2.8. Comparison 2: HFNC versus NIPPV or NIV, Outcome 8: Long-term respiratory effects: $\mathrm{PaO}_{2} / \mathrm{FiO}_{2}(\mathrm{mmHg})$

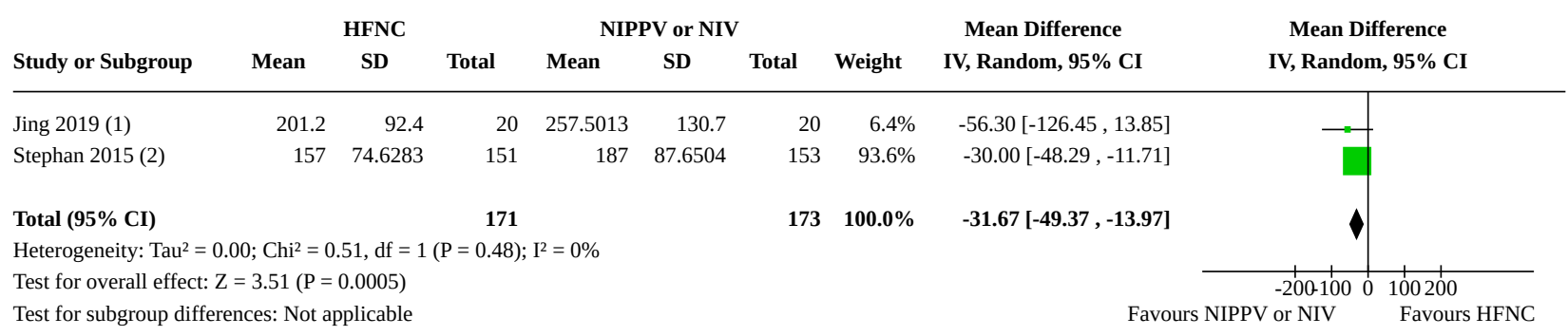

Footnotes

(1) At 48 hours

(2) AT 3 days

Analysis 2.9. Comparison 2: HFNC versus NIPPV or NIV, Outcome 9: Short-term respiratory effects: $\mathrm{PaO}_{2}$ (mmHg)

\begin{tabular}{|c|c|c|c|c|c|c|c|c|}
\hline \multirow[b]{2}{*}{ Study or Subgroup } & \multicolumn{3}{|c|}{ HFNC } & \multicolumn{3}{|c|}{ NIV } & \multicolumn{2}{|r|}{ Mean Difference } \\
\hline & Mean & SD & Total & Mean & SD & Total & Weight & IV, Random, 95\% CI \\
\hline Cong 2019 (1) & 72.16 & 17.53 & 84 & 71.99 & 17.49 & 84 & $54.0 \%$ & $0.17[-5.13,5.47]$ \\
\hline Frat 2015 (2) & 90 & 35 & 106 & 111 & 59 & 110 & $46.0 \%$ & $-21.00[-33.88,-8.12]$ \\
\hline Total (95\% CI) & & & 190 & & & 194 & $100.0 \%$ & $-9.57[-30.25,11.11$ \\
\hline
\end{tabular}

Heterogeneity: Tau $^{2}=198.83 ; \mathrm{Chi}^{2}=8.87, \mathrm{df}=1(\mathrm{P}=0.003) ; \mathrm{I}^{2}=89 \%$

Test for overall effect: $\mathrm{Z}=0.91(\mathrm{P}=0.36)$

Test for subgroup differences: Not applicable

Mean Difference

Footnotes

(1) At 12 hours

(2) At 6 hours 


\section{Analysis 2.10. Comparison 2: HFNC versus NIPPV or NIV, Outcome} 10: Short-term and long-term respiratory effects: $\mathrm{PaCO}_{2}(\mathrm{mmHg})$

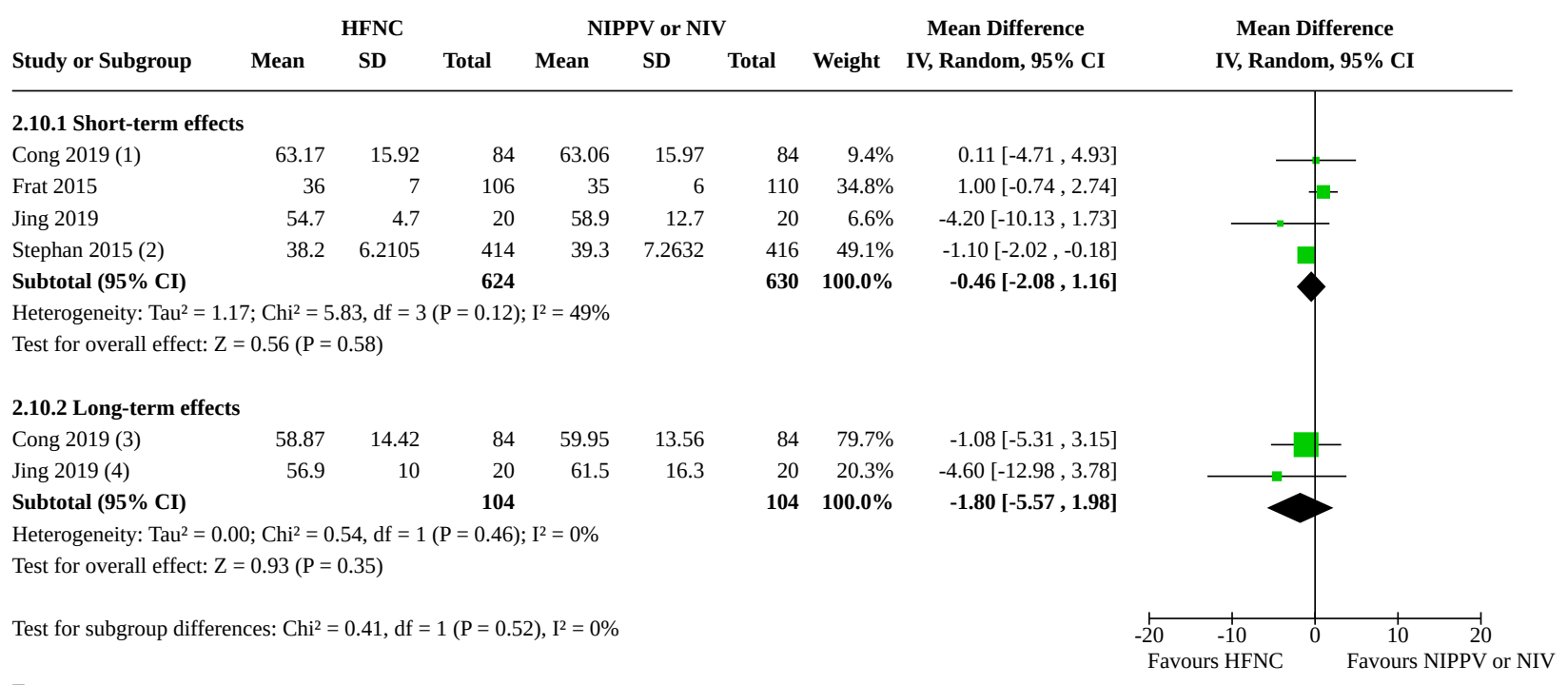

Footnotes
(1) At 12 hours
(2) At 6 to 12 hours
(3) At 5 days
(4) At 48 hours

\section{Analysis 2.11. Comparison 2: HFNC versus NIPPV or NIV, Outcome 11: Short-term respiratory effects: breaths/min}

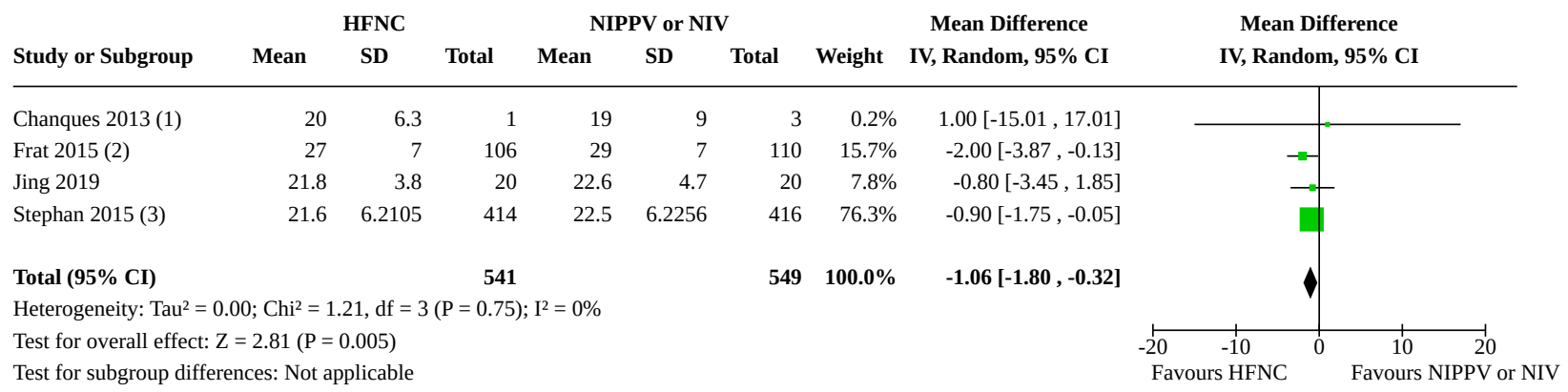

\section{Footnotes}

(1) Measured at end of treatment; data for Optiflow in HFNC group

(2) At 6 hours

(3) at 6-12 hours 
Analysis 2.12. Comparison 2: HFNC versus NIPPV or NIV, Outcome 12: Dyspnoea (any improvement)

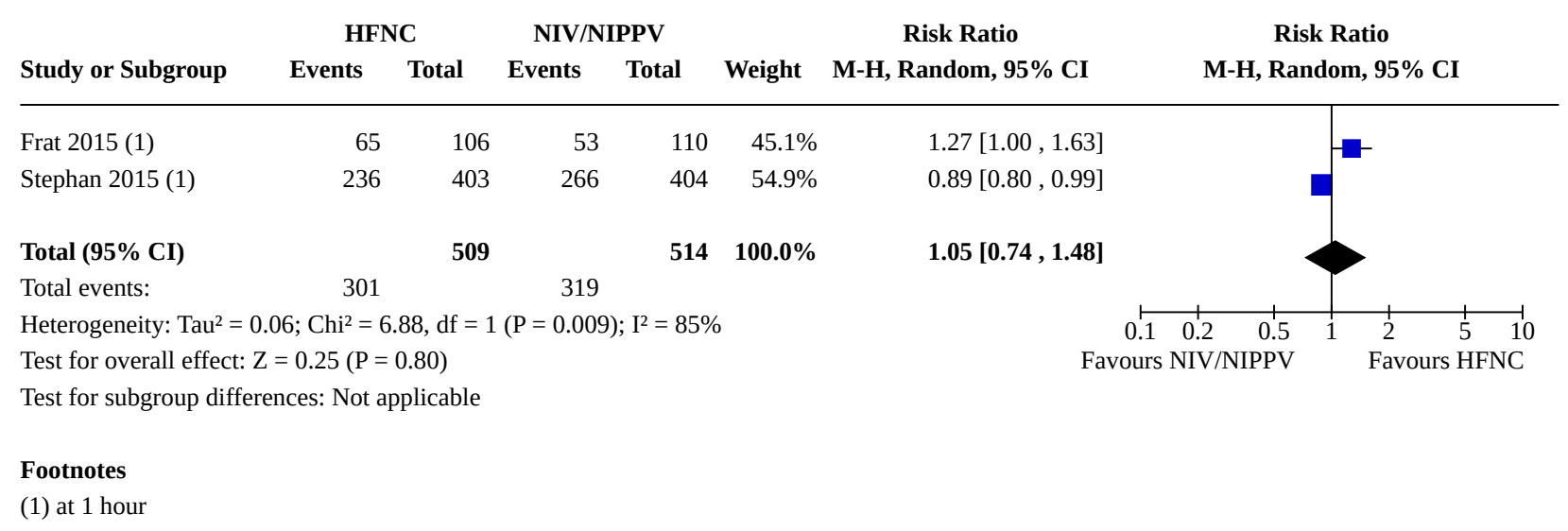

\section{ADDITIONAL TABLES}

Table 1. Comparison 1 (HFNC vs standard oxygen therapy): continuous outcomes from single studies

\begin{tabular}{|c|c|c|c|c|c|}
\hline Important outcomes & HFNC & $\begin{array}{l}\text { Standard oxygen } \\
\text { therapy }\end{array}$ & $\begin{array}{l}\text { Effect esti- } \\
\text { matea }\end{array}$ & P values ${ }^{\mathbf{b}}$ & Study ID \\
\hline Length of ICU stay (days) & $\begin{array}{l}\text { Median (IQR): } 8 \text { (4 } \\
\text { to } 14)\end{array}$ & $\begin{array}{l}\text { Median (IQR): } 6 \text { (4 } \\
\text { to } 13 \text { ) }\end{array}$ & & 0.07 & Azoulay 2018 \\
\hline Length of ICU stay (days) & $\begin{array}{l}\text { Median (IQR): } 6 \text { (4 } \\
\text { to } 16)\end{array}$ & $\begin{array}{l}\text { Median (IQR): } 5 \text { (3 } \\
\text { to } 13)\end{array}$ & & 0.53 & Futier 2016 \\
\hline Length of ICU stay (days) & $\begin{array}{l}\text { Median (IQR): } 6 \text { (2 } \\
\text { to } 8)\end{array}$ & $\begin{array}{l}\text { Median (IQR): } 6 \text { (2 } \\
\text { to 9) }\end{array}$ & & Not reported & $\begin{array}{l}\text { Hernandez } \\
\text { 2016b }\end{array}$ \\
\hline Length of ICU stay (days) & $\begin{array}{l}\text { Median (IQR): } 10 \\
\text { (7 to } 13 \text { ) }\end{array}$ & $\begin{array}{l}\text { Median (IQR): } 9 \text { (6 } \\
\text { to } 12 \text { ) }\end{array}$ & & 0.453 & Hu 2020 \\
\hline Length of ICU stay (days) & $\begin{array}{l}\text { Median (IQR): } 1 \text { (1 } \\
\text { to } 2 \text { ) }\end{array}$ & $\begin{array}{l}\text { Median (IQR): } 1 \text { (1 } \\
\text { to } 2 \text { ) }\end{array}$ & & 0.949 & Zochios 2018 \\
\hline $\begin{array}{l}\text { Short-term oxygenation }\left(\mathrm{PaO}_{2} /\right. \\
\left.\mathrm{FiO}_{2}\right)\end{array}$ & $\begin{array}{l}\text { Median (IQR):150 } \\
\text { (104 to 230) }\end{array}$ & $\begin{array}{l}\text { Median (IQR):119 } \\
\text { (86 to } 165)\end{array}$ & & $\begin{array}{l}\text { P value not re- } \\
\text { ported. Study } \\
\text { authors de- } \\
\text { scribed differ- } \\
\text { ence as signif- } \\
\text { icantly high- } \\
\text { er in the HFNC } \\
\text { group }\end{array}$ & Azoulay 2018 \\
\hline $\begin{array}{l}\text { Short-term comfort (at } 120 \text { min- } \\
\text { utes) }\end{array}$ & $\begin{array}{l}\text { Median (IQR): } 3 \text { (1 } \\
\text { to 5) }\end{array}$ & $\begin{array}{l}\text { Median (IQR): } 3 \text { (0 } \\
\text { to 5) }\end{array}$ & & 0.88 & Lemiale 2015 \\
\hline \multicolumn{6}{|l|}{$\begin{array}{l}\text { Scale of } 0 \text { to } 10(0=\text { absence of dis- } \\
\text { comfort, } 10=\text { worst possible dis- } \\
\text { comfort })\end{array}$} \\
\hline Long-term comfort (at 24 hours) & $\begin{array}{l}\text { Median (IQR): } 3 \text { (3 } \\
\text { to } 4.5 \text { ) }\end{array}$ & $\begin{array}{l}\text { Median (IQR): } 7 \text { (6 } \\
\text { to } 8 \text { ) }\end{array}$ & & $<0.001$ & Song 2017 \\
\hline
\end{tabular}


Table 1. Comparison 1 (HFNC vs standard oxygen therapy): continuous outcomes from single studies (Continued) Scale of 0 to 10 ( $0=$ no discomfort, $10=$ maximum discomfort)

\begin{tabular}{|c|c|c|c|c|c|}
\hline Additional outcomes & HFNC & $\begin{array}{l}\text { Standard oxygen } \\
\text { therapy }\end{array}$ & $\begin{array}{l}\text { Effect esti- } \\
\text { mate }^{a}\end{array}$ & $\mathbf{P}$ values $\mathbf{b}$ & Study ID \\
\hline $\begin{array}{l}\text { Duration of respiratory support } \\
\text { (hours) }\end{array}$ & $\begin{array}{l}\text { Mean (SD): } 59.0( \pm \\
30.8)\end{array}$ & $\begin{array}{l}\text { Mean (SD): } 65.0( \pm \\
41.6)\end{array}$ & $\begin{array}{l}\text { MD }(95 \% \mathrm{Cl}) \\
-6.00(-13.77 \\
\text { to } 1.77)\end{array}$ & 0.13 & Parke 2013a \\
\hline $\begin{array}{l}\text { Atelectasis (radiological atelectasis } \\
\text { score) }\end{array}$ & $\begin{array}{l}\text { Day 1: median } \\
\text { (IQR): } 2 \text { (1.5 to } 2.5) \\
\text { Day 5: median } \\
\text { (IQR): } 2 \text { (1.5 to } 2.5)\end{array}$ & $\begin{array}{l}\text { Day 1: median } \\
\text { (IQR): } 2 \text { ( } 1.5 \text { to } 3) \\
\text { Day 5: median } \\
\text { (IQR:) } 2 \text { ( } 1 \text { to } 2.5 \text { ) }\end{array}$ & & $\begin{array}{l}\text { Day 1: } 0.70 \\
\text { Day 5: } 0.15\end{array}$ & Corley 2014 \\
\hline Atelectasis (chest X-ray) & $\begin{array}{l}\text { Day 1: mean (SD): } \\
4.8( \pm 1.9) \\
\text { Day 3: mean }(S D): \\
4.8( \pm 1.9)\end{array}$ & $\begin{array}{l}\text { Day } 1 \text { : mean }(\mathrm{SD}) \\
4.9( \pm 1.8) \\
\text { Day } 3 \text { mean }(\mathrm{SD}) \\
4.7( \pm 2.1)\end{array}$ & & $\begin{array}{l}\text { Day 1: } 0.63 \\
\text { Day 3: } 0.69\end{array}$ & Parke 2013a \\
\hline $\begin{array}{l}\text { Long-term } \mathrm{PaCO}_{\mathbf{2}} \text { (at } 48 \text { hours; } \\
\mathrm{mmHg} \text { ) }\end{array}$ & $\begin{array}{l}\text { Mean (SD): } 41.3( \pm \\
7.5)\end{array}$ & $\begin{array}{l}\text { Mean (SD): } 37.2( \pm \\
9.6)\end{array}$ & $\begin{array}{l}\text { MD } 4.10,95 \% \\
\mathrm{Cl}-0.43 \text { to } 8.63\end{array}$ & & Hu 2020 \\
\hline $\begin{array}{l}\text { Short-term respiratory rate (at } 6 \\
\text { hours; breaths per minute) }\end{array}$ & $\begin{array}{l}\text { Median (IQR): } 25 \\
\text { (20 to } 30)\end{array}$ & $\begin{array}{l}\text { Median (IQR): } 26 \\
\text { ( } 21 \text { to } 31 \text { ) }\end{array}$ & & Not reported & Azoulay 2018 \\
\hline $\begin{array}{l}\text { Long-term respiratory rate (at } 120 \\
\text { minutes; breaths per minute) }\end{array}$ & $\begin{array}{l}\text { Median (IQR): } 25 \\
\text { (22 to } 29)\end{array}$ & $\begin{array}{l}\text { Median (IQR) } 25 \\
\text { (21 to } 31)\end{array}$ & & Not reported & Lemiale 2015 \\
\hline Length of hospital stay (days) & $\begin{array}{l}\text { Median (IQR): } 24 \\
\text { (14 to } 40)\end{array}$ & $\begin{array}{l}\text { Median (IQR): } 27 \\
\text { (15 to } 42 \text { ) }\end{array}$ & & 0.60 & Azoulay 2018 \\
\hline Length of hospital stay (days) & $\begin{array}{l}\text { Median (IQR): } 12 \\
\text { (7 to } 20)\end{array}$ & $\begin{array}{l}\text { Median (IQR): } 11 \\
\text { (7 to } 18 \text { ) }\end{array}$ & & 0.58 & Futier 2016 \\
\hline Length of hospital stay (days) & $\begin{array}{l}\text { Median (IQR): } 11(6 \\
\text { to } 15)\end{array}$ & $\begin{array}{l}\text { Median (IQR): } 12 \\
\text { ( } 6 \text { to } 16)\end{array}$ & & 0.76 & $\begin{array}{l}\text { Hernandez } \\
2016 b\end{array}$ \\
\hline Length of hospital stay (days) & $\begin{array}{l}\text { Median (IQR): } 7 \text { (6 } \\
\text { to 9) }\end{array}$ & $\begin{array}{l}\text { Median (IQR): } 9 \text { ( } 7 \\
\text { to } 6)\end{array}$ & & 0.012 & Zochios 2018 \\
\hline $\begin{array}{l}\text { Participant-reported outcomes } \\
\text { Dyspnoea } \\
\text { Modified Borg scale ( } 0=\text { no dysp- } \\
\text { noea, } 10=\text { maximal dyspnoea }\end{array}$ & $\begin{array}{l}\text { Median (IQR): } 1 \text { (0 } \\
\text { to 3) }\end{array}$ & $\begin{array}{l}\text { Median (IQR): } 0 \text { (0 } \\
\text { to } 1 \text { ) }\end{array}$ & & 0.008 & Corley 2014 \\
\hline $\begin{array}{l}\text { Participant-reported outcomes } \\
\text { Dyspnoea } \\
\text { Scale of } 0 \text { to } 10 \text { ( } 0=\text { absence of dysp- } \\
\text { noea, } 10=\text { worst possible dyspnoea) }\end{array}$ & $\begin{array}{l}\text { Median (IQR): } 3 \text { (2 } \\
\text { to } 6)\end{array}$ & $\begin{array}{l}\text { Median (IQR): } 3 \text { ( } 5 \\
\text { to } 9 \text { ) }\end{array}$ & & 0.40 & Lemiale 2015 \\
\hline $\begin{array}{l}\text { Participant-reported outcomes } \\
\text { Dyspnoea }\end{array}$ & $\begin{array}{l}\text { Mean (SD): } 1.6 \\
(1.2)\end{array}$ & $\begin{array}{l}\text { Mean }(S D): 2.9 \\
(1.5)\end{array}$ & $\begin{array}{l}\mathrm{MD}-1.3,95 \% \\
\mathrm{Cl}-2.60 \text { to } 0.00\end{array}$ & 0.04 & $\begin{array}{l}\text { Rittayamai } \\
2014\end{array}$ \\
\hline
\end{tabular}


Table 1. Comparison 1 (HFNC vs standard oxygen therapy): continuous outcomes from single studies (Continued) Scale of 0 to 10 ( $0=$ no dyspnoea, 10 = maximal dyspnoea). Authors reported proportion of patients with improvement

\begin{tabular}{|c|c|c|c|c|c|}
\hline $\begin{array}{l}\text { Participant-reported outcomes } \\
\text { Dry mouth }\end{array}$ & Mean (SD) $3.6(2.5)$ & Mean (SD) 5 (3.1) & $\begin{array}{l}\mathrm{MD}-1.40,95 \% \\
\mathrm{Cl}-2.68 \text { to } \\
-0.12\end{array}$ & 0.016 & Maggiore 2014 \\
\hline $\begin{array}{l}\text { Scale of } 0 \text { to } 10(0=\text { no dryness, } 10= \\
\text { maximum dryness) }\end{array}$ & & & & & \\
\hline $\begin{array}{l}\text { Cost comparison of treatment } \\
\text { Total hospitalization expenditure, } \$\end{array}$ & $\begin{array}{l}\text { Mean (SD): } \\
11522.65(762.45)\end{array}$ & $\begin{array}{l}\text { Mean (SD): } \\
12219.73(1028.66)\end{array}$ & & 0.001 & Yu 2017 \\
\hline
\end{tabular}

acalculated using RevMan Web 2019

bas reported by study authors

$\mathrm{Cl}$ : confidence interval

HFNC: high-flow nasal cannulae

ICU: intensive care unit

IQR: interquartile range

$M D$ : mean difference

$\mathrm{PaO}_{2} / \mathrm{FiO}_{2}$ : ratio of partial pressure of arterial oxygen to fraction of inspired oxygen

$\mathrm{PaCO}_{2}$ : partial pressure of carbon dioxide in arterial blood

SD: standard deviation

Table 2. Comparison 1 (HFNC vs standard oxygen therapy): dichotomous data from studies not included in metaanalysis

\begin{tabular}{|c|c|c|c|c|}
\hline Additional outcomes & $\begin{array}{l}\text { HFNC } \\
n / N\end{array}$ & $\begin{array}{l}\text { Standard oxy- } \\
\text { gen therapy } \\
\mathrm{n} / \mathrm{N}\end{array}$ & Effect estimate ${ }^{a}$ & Study \\
\hline Atelectasis & $2 / 56$ & $5 / 54$ & $\begin{array}{l}\mathrm{RR} 0.39,95 \% \mathrm{Cl} 0.08 \text { to } \\
1.90\end{array}$ & Yu 2017 \\
\hline $\begin{array}{l}\text { Adverse events } \\
\text { Ventilator-acquired tracheobronchitis }\end{array}$ & $3 / 264$ & $7 / 263$ & $\begin{array}{l}\text { RR } 0.43,95 \% \mathrm{Cl} 0.11 \text { to } \\
1.63\end{array}$ & $\begin{array}{l}\text { Hernandez } \\
2016 b\end{array}$ \\
\hline $\begin{array}{l}\text { Adverse events } \\
\text { Abdominal distension }\end{array}$ & $3 / 56$ & $0 / 54$ & $\begin{array}{l}\text { RR } 6.75,95 \% \mathrm{Cl} 0.36 \text { to } \\
127.76\end{array}$ & Yu 2017 \\
\hline $\begin{array}{l}\text { Participant-reported outcomes } \\
\text { Dyspnoea (any improvement; using categor- } \\
\text { ical data reported as marked improvement, } \\
\text { slight improvement, no change, slight deteri- } \\
\text { oration, marked deterioration) }\end{array}$ & $65 / 106$ & $31 / 94$ & $\begin{array}{l}\text { RR } 1.86,95 \% \mathrm{Cl} 1.34 \text { to } \\
2.57\end{array}$ & Frat 2015 \\
\hline $\begin{array}{l}\text { Participant-reported outcomes } \\
\text { Dry mouth (data included dry mouth, nose, or } \\
\text { throat) }\end{array}$ & $18 / 47$ & $30 / 43$ & $\begin{array}{l}\mathrm{RR} 0.55,95 \% \mathrm{Cl} 0.36 \text { to } \\
0.83\end{array}$ & $\begin{array}{l}\text { Vourc'h } 2020 \\
\text { Vourc'h } 2020\end{array}$ \\
\hline
\end{tabular}


Table 2. Comparison 1 (HFNC vs standard oxygen therapy): dichotomous data from studies not included in metaanalysis (Continued)

\begin{tabular}{|c|c|c|c|}
\hline Participant-reported outcomes & $1 / 56$ & $7 / 54$ & $\begin{array}{l}\text { RR } 0.14,95 \% \mathrm{Cl} 0.02 \text { to } \\
1.08\end{array}$ \\
\hline
\end{tabular}

Throat and nasal pain

acalculated using RevMan Web 2019

$\mathrm{Cl}$ : confidence interval

HFNC: high-flow nasal cannulae

$\mathrm{N}$ : total number of participants per group

n: number of participants who had an event

RR: risk ratio

Table 3. Comparison 1 (HFNC vs standard oxygen therapy): summary effects of additional outcomes

\begin{tabular}{|c|c|c|c|}
\hline Outcome & Study IDs & $\begin{array}{l}\text { Effect estimate } \\
\text { (short-term) }\end{array}$ & $\begin{array}{l}\text { Effect estimate } \\
\text { (long-term) }\end{array}$ \\
\hline $\begin{array}{l}\text { Duration of res- } \\
\text { piratory sup- } \\
\text { port }\end{array}$ & Parke 2013a & $\begin{array}{l}\text { MD }-6.00 \text { hours, } 95 \% \mathrm{Cl}-13.77 \text { to } \\
1.77 ; 1 \text { study, } 340 \text { participants; Table } \\
1\end{array}$ & - \\
\hline
\end{tabular}

\begin{tabular}{lll}
\hline Long-term & Maggiore 2014; & MD 27.97, 95\% \\
$\mathbf{P a O}_{\mathbf{2}} / \mathrm{FiO}_{\mathbf{2}}$ & Vourc'h 2020 & $\mathrm{Cl} 5.60$ to $50.33 ;$ \\
& & 2 studies, 195 \\
& & participants; $12=$ \\
& & $81 \% ;$ Analysis 1.7
\end{tabular}

\begin{tabular}{|c|c|c|c|}
\hline Atelectasis & Yu 2017 & $\begin{array}{l}\text { RR } 0.39,95 \% \text { Cl } 0.08 \text { to } 1.90 ; 1 \text { study; } \\
99 \text { participants; Table } 2\end{array}$ & $\begin{array}{l}\text { Additional data available from } \\
2 \text { studies (Corley 2014; Parke } \\
\text { 2013a)a; see Table } 1\end{array}$ \\
\hline
\end{tabular}

\begin{tabular}{llll}
\hline $\mathbf{P a O}_{2}$ & Frat 2015; $\mathrm{Hu}$ & $\mathrm{MD} 4.92 \mathrm{mmHg}, 95 \% \mathrm{Cl}-1.24$ to & $\mathrm{MD} 12.27 \mathrm{mmHg}$, \\
2020; Parke 2011; & $11.07 ; 4$ studies, 415 participants; $2^{2}$ & $95 \% \mathrm{Cl} 7.51$ to \\
Maggiore 2014; & $=47 \% ;$ Analysis 1.8 & $17.04 ; 2$ studies, \\
Song 2017 & & 644 participants; \\
& & $12=0 \%$; Analysis \\
&
\end{tabular}

\begin{tabular}{|c|c|c|c|c|}
\hline $\mathrm{SpO}_{2}$ & $\begin{array}{l}\text { Maggiore 2014; } \\
\text { Parke 2011; } \\
\text { Parke 2013a; Rit- } \\
\text { tayamai 2014; } \\
\text { Song } 2017\end{array}$ & $\begin{array}{l}\text { MD } 0.79 \%, 95 \% \mathrm{Cl}-0.29 \text { to } 1.88 ; 5 \\
\text { studies, } 572 \text { participants; }\left.\right|^{2}=88 \% \text {; } \\
\text { Analysis } 1.9\end{array}$ & $\begin{array}{l}\text { MD } 1.28 \%, 95 \% \\
\text { CI } 0.02 \text { to } 2.55 ; \\
2 \text { studies, } 445 \\
\text { participants; } 12= \\
81 \% ; \text { Analysis } 1.9\end{array}$ & $\begin{array}{l}\text { Long-term effect estimate was } \\
\text { significant }(P=0.05) \text {, howev- } \\
\text { er, the high number of compar- } \\
\text { isons in this review limits our in- } \\
\text { terpretation of this result. }\end{array}$ \\
\hline
\end{tabular}

\begin{tabular}{|c|c|c|c|c|}
\hline $\mathrm{PaCO}_{2}$ & $\begin{array}{l}\text { Frat 2015 Frat } \\
\text { 2015; Hernan- } \\
\text { dez 2016b; Hu } \\
\text { 2020; Maggiore } \\
\text { 2014; Parke 2011; } \\
\text { Parke 2013a; } \\
\text { Song } 2017\end{array}$ & $\begin{array}{l}\text { MD }-1.05 \mathrm{mmHg}, 95 \% \mathrm{Cl}-2.24 \text { to } \\
-0.13 ; 5 \text { studies, } 755 \text { participants; }\left.\right|^{2} \\
=28 \% \text {; Analysis } 1.10\end{array}$ & $\begin{array}{l}\text { MD } 4.10 \mathrm{mmHg} \text {, } \\
95 \% \mathrm{Cl}-0.43 \text { to } \\
8.63 ; 1 \text { study, } 56 \\
\text { participants; Ta- } \\
\text { ble } 1\end{array}$ & \\
\hline Respiratory rate & $\begin{array}{l}\text { Corley 2014; } \\
\text { Frat 2015; Hu } \\
\text { 2020; Maggiore }\end{array}$ & $\begin{array}{l}\text { MD }-2.02 \text { breaths } / \text { min, } 95 \% \mathrm{Cl}-3.66 \\
\text { to }-0.37 ; 7 \text { studies, } 1017 \text { partici- } \\
\text { pants; }\left.\right|^{2}=87 \% \text {; Analysis } 1.11\end{array}$ & $\begin{array}{l}\text { MD }-2.01 \\
\text { breaths/min, } \\
95 \% \mathrm{Cl}-4.39 \text { to }\end{array}$ & $\begin{array}{l}\text { Additional data available from } 2 \\
\text { studies (Azoulay 2018; Lemiale } \\
\text { 2015)a; see Table } 1\end{array}$ \\
\hline
\end{tabular}


Table 3. Comparison 1 (HFNC vs standard oxygen therapy): summary effects of additional outcomes (Continued)
2014; Parke 2011;
0.37 ; 4 studies,
Parke 2013a; Rit-
591 participants;
tayamai 2014;
Song 2017;
$12=92 \%$; Analy-
Vourc'h 2020
sis 1.11

\section{Additional ad- \\ ventilator-ac- \\ quired tracheo- \\ bronchitis}

verse events: $2016 \mathrm{~b}$

RR $0.43,95 \%$ Cl 0.11 to 1.63 ; 1 study,

\begin{tabular}{ll}
\hline $\begin{array}{l}\text { Additional ad- } \\
\text { verse events: } \\
\text { abdominal dis- }\end{array}$ & Yu $2017 \quad$ RR $6.75,95 \%$ Cl 0.36 to $127.76 ; 1$ \\
tension & study, 110 participants; Table 2 \\
\hline
\end{tabular}

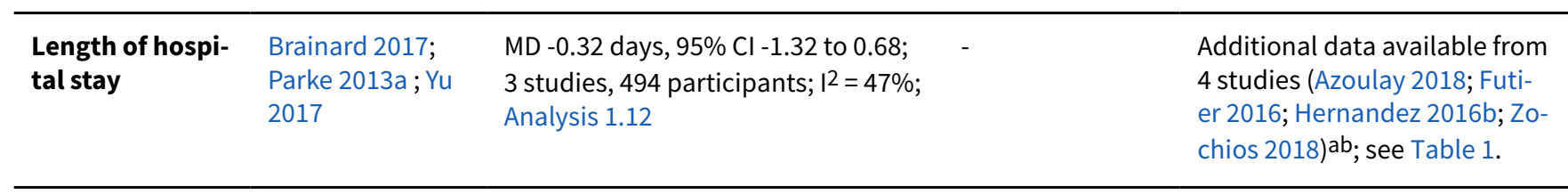

\begin{tabular}{lll}
\hline $\begin{array}{l}\text { Other partici- } \\
\text { pant-reported } \\
\text { outcomes }\end{array}$ & $\begin{array}{l}\text { Frat 2015; Rit- } \\
\text { tayamai } 2014\end{array}$ & $\begin{array}{l}\text { MD }-1.30,95 \% \mathrm{Cl}-2.60 \text { to } 0.00 ; 1 \\
\text { study, } 17 \text { participants; Table } 1\end{array}$ \\
& & RR $1.86,95 \% \mathrm{Cl} 1.34$ to $2.57 ; 1$ study, \\
Dyspnoea & & 200 participant; Table 2
\end{tabular}

Additional data available from 3 studies (Corley 2014; Lemiale 2015; Rittayamai 2014)a; see Table 1.

Azoulay 2018 data reported in figures from which numerical data could not be extracted. Study authors reported no significant difference between groups.

\begin{tabular}{lll}
\hline $\begin{array}{l}\text { Other partici- } \\
\text { pant-reported } \\
\text { outcomes }\end{array}$ & $\begin{array}{l}\text { Maggiore 2014; } \\
\text { Vourc'h } 2020\end{array}$ & RR 0.55, 95\% Cl 0.36 to $0.83 ; 1$ study \\
& & MD participants; Table 2 \\
Dry mouth & & study, 80 participants; Table 1
\end{tabular}

Additional data available from Maggiore 2014 reported in Table 1. Additional data from Vourc'h 2020 reported in Table 2.

Cuquemelle 2012 effect size was not reported but the authors stated there was no evidence of a difference between groups.

\begin{tabular}{|c|c|c|c|}
\hline $\begin{array}{l}\text { Other partici- } \\
\text { pant-reported } \\
\text { outcomes } \\
\text { Throat or nasal } \\
\text { pain }\end{array}$ & Yu 2017 & $\begin{array}{l}\text { RR } 0.14,95 \% \text { Cl } 0.02 \text { to } 1.08 ; 1 \text { study, } \\
110 \text { participants; Table } 2\end{array}$ & - \\
\hline $\begin{array}{l}\text { Other partici- } \\
\text { pant-reported } \\
\text { outcomes }\end{array}$ & Futier 2016 & $\begin{array}{l}\text { RR } 17.62,95 \% \text { Cl } 1.03 \text { to } 301.65 ; 1 \\
\text { study, } 220 \text { participants; Table } 2\end{array}$ & - \\
\hline $\begin{array}{l}\text { Treatment with- } \\
\text { drawn due to } \\
\text { discomfort }\end{array}$ & & & \\
\hline
\end{tabular}


Table 3. Comparison 1 (HFNC vs standard oxygen therapy): summary effects of additional outcomes (Continued)

\begin{tabular}{|c|c|c|c|c|}
\hline $\begin{array}{l}\text { Other partici- } \\
\text { pant-reported } \\
\text { outcomes } \\
\text { Refusal to con- } \\
\text { tinue treatment }\end{array}$ & $\begin{array}{l}\text { Futier 2016; } \\
\text { Parke 2013a }\end{array}$ & $\begin{array}{l}\text { RR } 26.89,95 \% \mathrm{Cl} 3.67 \text { to } 197.32 ; 2 \\
\text { studies, } 560 \text { participants; Analysis } \\
1.13\end{array}$ & - & $\begin{array}{l}\text { Azoulay } 2018 \text { reported partici- } \\
\text { pant discontinuation in HFNC } \\
\text { group due to discomfort, but it } \\
\text { was unclear whether any partic- } \\
\text { ipants in the control group dis- } \\
\text { continued due to discomfort. }\end{array}$ \\
\hline $\begin{array}{l}\text { Cost compar- } \\
\text { ison of treat- } \\
\text { ment }\end{array}$ & Yu 2017 & - & - & $\begin{array}{l}\text { Mean costs reported for HFNC } \\
\text { group only. See Table } 1\end{array}$ \\
\hline \multicolumn{5}{|c|}{$\begin{array}{l}\text { aWe did not combine data from these studies in analyses because data were reported as median values, or did not include relevant } \\
\text { distribution variables for meta-analysis with other studies } \\
\text { bFrom visual inspection, we noted that these data were likely to be right skewed due to the comparable magnitudes of the mean and } \\
\text { standard deviation. This is expected for outcomes such a length of hospital stay due to most participants being discharged in a short } \\
\text { time period with some outliers staying significantly longer. However, right skew introduces artefact into calculation of the effect esti- } \\
\text { mate, limiting the interpretation of the result. }\end{array}$} \\
\hline
\end{tabular}

$\mathrm{Cl}$ : confidence interval

FiO2: fraction of inspired oxygen

HFNC: high flow nasal cannula(e)

MD: mean difference

$\mathrm{PaCO}_{2}$ : carbon dioxide clearance

$\mathrm{PaO}_{2}$ : partial pressure of arterial oxygen

RR: risk ratio

$\mathrm{SpO}_{2}$ : oxygen saturation

Table 4. Comparison 1 (HFNC vs standard oxygen therapy): sensitivity analysis

\begin{abstract}
Risk of selection: studies excluded from primary analysis owing to high or unclear risk of selection bias for random sequence generation or allocation concealment
\end{abstract}

\begin{tabular}{|c|c|c|}
\hline Important outcomes & Excluded studies & Effect of sensitivity analysis \\
\hline Failure of treatment & $\begin{array}{l}\text { Frat 2015; Hu 2020; Lemiale 2015; Maggiore } \\
\text { 2014; Song 2017; Yu } 2017\end{array}$ & $\begin{array}{l}\text { Effect estimate no longer indicated improvement } \\
\text { with HFNC use (RR } 0.85,95 \% \mathrm{Cl} 0.62 \text { to } 1.17 ; 9 \text { stud- } \\
\text { ies, } 2457 \text { participants; } I^{2}=55 \% \text { ) }\end{array}$ \\
\hline In-hospital mortality & Frat 2015; Hu 2020; Maggiore 2014; Yu 2017 & $\begin{array}{l}\text { Interpretation of the effect estimate remained the } \\
\text { same }\end{array}$ \\
\hline $\begin{array}{l}\text { Important adverse } \\
\text { events: pneumonia }\end{array}$ & Frat 2015; Yu 2017 & $\begin{array}{l}\text { Interpretation of the effect estimate remained the } \\
\text { same }\end{array}$ \\
\hline $\begin{array}{l}\text { Important adverse } \\
\text { events: nasal mucosa or } \\
\text { skin trauma }\end{array}$ & - & - \\
\hline Length of ICU stay & Brainard 2017; Frat 2015; Maggiore 2014; Yu 2017 & $\begin{array}{l}\text { Interpretation of the effect estimate remained the } \\
\text { same }\end{array}$ \\
\hline $\mathrm{PaO}_{2} / \mathrm{FiO}_{2}$ up to 24 hours & Frat 2015; Maggiore 2014; Parke 2011 & $\begin{array}{l}\text { Effect estimate indicated higher } \mathrm{PaO}_{2} / \mathrm{FiO}_{2} \text { when } \\
\text { standard oxygen therapy was used (MD } 25.28 \\
\mathrm{mmHg}, 95 \% \mathrm{Cl} 7.23 \text { to } 43.32 ; 2 \text { studies, } 245 \text { partici- } \\
\text { pants; } \mathrm{I}^{2}=0 \% \text { ) }\end{array}$ \\
\hline
\end{tabular}




\section{Table 4. Comparison 1 (HFNC vs standard oxygen therapy): sensitivity analysis (Continued)}

\begin{tabular}{lll} 
Comfort (short-term) & Frat 2015; Maggiore 2014; Rittayamai 2014 & $\begin{array}{l}\text { Interpretation of the effect estimate remained the } \\
\text { same }\end{array}$ \\
\hline Comfort (long-term) & Maggiore 2014; & $\begin{array}{l}\text { Interpretation of the effect estimate remained the } \\
\text { same }\end{array}$
\end{tabular}

\section{High risks of other bias: studies excluded from primary analysis owing to high risks of other bias}

\begin{tabular}{lll}
\hline Outcome & Excluded studies & Effect of sensitivity analysis \\
\hline Failure of treatment & $\begin{array}{l}\text { Fernandez 2017; Hu 2020; Parke 2011; Parke } \\
\text { 2013a; Zochios 2018 (selective reporting bias) } \\
\end{array}$ & $\begin{array}{l}\text { Frat 2015 (selective reporting bias, and differ- } \\
\text { ences in treatment in the HFNC group) }\end{array}$ \\
\hline same & \\
\hline In-hospital mortality & $\begin{array}{l}\text { Fernandez 2017; Frat 2015; Hu 2020; Parke } \\
\text { 2013a; Zochios 2018 (selective reporting bias) }\end{array}$ & $\begin{array}{l}\text { Interpretation of the effect estimate remained the } \\
\text { same }\end{array}$ \\
& $\begin{array}{l}\text { Frat 2015 (differences in treatment in the HFNC } \\
\text { group) }\end{array}$ & \\
\hline $\begin{array}{l}\text { Important adverse } \\
\text { events: pneumonia }\end{array}$ & $\begin{array}{l}\text { Frat 2015 (selective reporting bias, and differ- } \\
\text { ences in treatment in the HFNC group) }\end{array}$ & $\begin{array}{l}\text { Interpretation of the effect estimate remained the } \\
\text { same }\end{array}$ \\
\hline
\end{tabular}

Important adverse

events: nasal mucosa or

skin trauma

\begin{tabular}{|c|c|c|}
\hline Length of ICU stay & $\begin{array}{l}\text { Brainard } 2017 \text { (attrition bias) } \\
\text { Frat } 2015 \text { (selective reporting bias, and differ- } \\
\text { ences in treatment in the HFNC group) } \\
\text { Parke } 2013 \text { a (selective reporting bias) }\end{array}$ & $\begin{array}{l}\text { Interpretation of the effect estimate remained the } \\
\text { same }\end{array}$ \\
\hline $\mathrm{PaO}_{2} / \mathrm{FiO}_{2}$ up to 24 hours & $\begin{array}{l}\text { Frat } 2015 \text { (selective reporting bias, and differ- } \\
\text { ences in treatment in the HFNC group) } \\
\text { Parke } 2011 \text { (selective reporting bias) }\end{array}$ & $\begin{array}{l}\text { Effect estimate indicated higher } \mathrm{PaO}_{2} / \mathrm{FiO}_{2} \text { when } \\
\text { standard oxygen therapy was used ( } \mathrm{MD} 29.28 \\
\text { mmHg, } 95 \% \mathrm{Cl} 13.86 \text { to } 44.70 ; 3 \text { studies, } 350 \text { partici- } \\
\text { pants; } \mathrm{I}^{2}=0 \% \text { ) }\end{array}$ \\
\hline Comfort (short-term) & $\begin{array}{l}\text { Frat } 2015 \text { (selective reporting bias, and differ- } \\
\text { ences in treatment in the HFNC group) } \\
\text { Parke } 2013 \text { a (selective reporting bias) }\end{array}$ & $\begin{array}{l}\text { Interpretation of the effect estimate remained the } \\
\text { same }\end{array}$ \\
\hline Comfort (long-term) & Parke 2013a (selective reporting bias) & $\begin{array}{l}\text { Effect estimate indicated improved comfort when } \\
\text { HFNC was used (MD }-2.10,95 \% \mathrm{Cl}-3.16 \text { to }-1.04 ; 1 \\
\text { study, } 105 \text { participants) }\end{array}$ \\
\hline
\end{tabular}

\section{Fixed effect versus random effects: we re-analysed the data using a fixed-effect model}

\begin{tabular}{ll}
\hline Outcomes & Effect of sensitivity analysis \\
\hline Failure of treatment $\quad$ Interpretation of the effect estimate for all outcomes remained the same \\
In-hospital mortality \\
$\begin{array}{l}\text { Important adverse } \\
\text { events: pneumonia }\end{array}$ \\
\hline
\end{tabular}


Table 4. Comparison 1 (HFNC vs standard oxygen therapy): sensitivity analysis (Continued)

Important adverse

events: nasal mucosa or

skin trauma

Length of ICU stay

$\mathrm{PaO}_{2} / \mathrm{FiO}_{2}$ up to 24 hours

Comfort (short-term)

Comfort (long-term)

Funding: studies excluded from analysis in which funding was from commercial sources

\begin{tabular}{|c|c|c|}
\hline Outcome & Excluded studies & Effect of sensitivity analysis \\
\hline Failure of treatment & $\begin{array}{l}\text { Azoulay 2018; Corley 2014; Frat 2015; Hernandez } \\
\text { 2016b; Lemiale 2015; Maggiore 2014; Parke 2011; } \\
\text { Parke 2013a; Zochios } 2018\end{array}$ & $\begin{array}{l}\text { Interpretation of the effect estimate remained the } \\
\text { same }\end{array}$ \\
\hline In-hospital mortality & $\begin{array}{l}\text { Azoulay 2018; Hernandez 2016b; Maggiore 2014; } \\
\text { Parke 2013a; Zochios } 2018\end{array}$ & $\begin{array}{l}\text { Interpretation of the effect estimate remained the } \\
\text { same }\end{array}$ \\
\hline $\begin{array}{l}\text { Important adverse } \\
\text { events: pneumonia }\end{array}$ & Frat 2015; Hernandez 2016b & $\begin{array}{l}\text { Interpretation of the effect estimate remained the } \\
\text { same }\end{array}$ \\
\hline $\begin{array}{l}\text { Important adverse } \\
\text { events: nasal mucosa or } \\
\text { skin trauma }\end{array}$ & Hernandez 2016b & $\begin{array}{l}\text { Interpretation of the effect estimate remained the } \\
\text { same }\end{array}$ \\
\hline Length of ICU stay & $\begin{array}{l}\text { Corley 2014; Frat 2015; Maggiore 2014; Parke } \\
\text { 2013a }\end{array}$ & $\begin{array}{l}\text { Interpretation of the effect estimate remained the } \\
\text { same }\end{array}$ \\
\hline $\mathrm{PaO}_{2} / \mathrm{FiO}_{2}$ up to 24 hours & $\begin{array}{l}\text { Corley 2014; Frat 2015; Maggiore 2014; Parke } \\
\text { 2013a }\end{array}$ & $\begin{array}{l}\text { Interpretation of the effect estimate remained the } \\
\text { same (only one study remaining in analysis) }\end{array}$ \\
\hline Comfort (short-term) & Frat 2015; Maggiore 2014; Parke 2013a & $\begin{array}{l}\text { Interpretation of the effect estimate remained the } \\
\text { same (only one study remaining in analysis) }\end{array}$ \\
\hline Comfort (long-term) & Maggiore 2014; Parke 2013a & No studies remaining in analysis \\
\hline
\end{tabular}

$\mathrm{Cl}$ : confidence interval

MD: mean difference

RR: risk ratio

$\mathrm{PaO}_{2} / \mathrm{FiO}_{2}$ : partial pressure of arterial oxygen/fraction of inspired oxygen

Table 5. Comparison 2 (HFNC vs NIV or NIPPV): dichotomous outcomes from single studies

\begin{tabular}{|c|c|c|c|c|}
\hline Important outcomes & HFNC $\mathbf{n} / \mathbf{N}$ & $\begin{array}{l}\text { NIV or NIPPV n/ } \\
\text { N }\end{array}$ & Effect estimate ${ }^{a}$ & Study ID \\
\hline Participant-reported outcomes & $74 / 84$ & $57 / 84$ & RR $1.30,95 \% \mathrm{Cl} 1.10$ to 1.53 & Cong 2019 \\
\hline \multicolumn{5}{|l|}{ Comfort } \\
\hline Adverse events & $8 / 414$ & $7 / 416$ & RR $1.15,95 \% \mathrm{Cl} 0.42$ to 3.14 & Stephan 2015 \\
\hline Pneumothorax & & & & \\
\hline
\end{tabular}


Table 5. Comparison 2 (HFNC vs NIV or NIPPV): dichotomous outcomes from single studies (Continued)

\begin{tabular}{lllll} 
Additional outcomes & HFNC $\mathbf{n} / \mathbf{N}$ & $\begin{array}{l}\text { NIV or NIPPV } \mathbf{n} / \\
\mathbf{N}\end{array}$ & Effect estimate & Study ID \\
\hline Adverse events & $11 / 290$ & $18 / 314$ & RR 0.66, 95\% Cl 0.32 to 1.38 Hernandez & $2016 \mathrm{~b}$ \\
Ventilator-acquired tracheobronchitis & & & \\
\hline acalculated using RevMan Web 2019 & & & \\
\hline
\end{tabular}

$\mathrm{Cl}$ : confidence interval

HFNC: high-flow nasal cannulae

$\mathrm{N}$ : total number of participants in the group

$\mathrm{n}$ : number of participants who had an event

$\mathrm{RR}$ : risk ratio

Table 6. Comparison 2 (HFNC vs NIV or NIPPV): continuous outcomes for single studies

\begin{tabular}{|c|c|c|c|c|c|}
\hline Important outcomes & HFNC & NIV or NIPPV & $\begin{array}{l}\text { Effect esti- } \\
\text { mate }\end{array}$ & P valueb & Study ID \\
\hline Length of ICU stay (days) & $\begin{array}{l}\text { Median (IQR): } 9 \text { (4 to } \\
\text { 19) }\end{array}$ & $\begin{array}{l}\text { Median (IQR): } 10.5 \text { ( } 5 \\
\text { to } 19)\end{array}$ & & Not reported & $\begin{array}{l}\text { Hernandez } \\
\text { 2016a }\end{array}$ \\
\hline Length of ICU stay (days) & $\begin{array}{l}\text { Median (IQR) } 6 \text { ( } 4 \text { to } \\
\text { 10) }\end{array}$ & $\begin{array}{l}\text { Median (IQR) } 6 \text { ( } 4 \text { to } \\
\text { 10) }\end{array}$ & & 0.77 & Stephan 2015 \\
\hline Short-term comfort (1 hour) & Poor: $16.7 \%$ & Poor: $17.8 \%$ & & 0.32 & Stephan 2015 \\
\hline $\begin{array}{l}\text { 5-point scale of 'poor', 'accept- } \\
\text { able', or 'good' }\end{array}$ & $\begin{array}{l}\text { Acceptable: } 31.0 \% \\
\text { Good: } 51.0 \%\end{array}$ & $\begin{array}{l}\text { Acceptable: } 29.3 \% \\
\text { Good: } 53.0 \%\end{array}$ & & & \\
\hline Long-term comfort (day 3) & Poor: $21 \%$ & Poor: $21 \%$ & & $>0.99$ & Stephan 2015 \\
\hline $\begin{array}{l}\text { 5-point scale of 'poor', 'accept- } \\
\text { able', or 'good' }\end{array}$ & $\begin{array}{l}\text { Acceptable: } 32.4 \% \\
\text { Good: } 47 \%\end{array}$ & $\begin{array}{l}\text { Acceptable: } 31 \% \\
\text { Good: } 48.3 \%\end{array}$ & & & \\
\hline Additional outcomes & HFNC & NIV or NIPPV & $\begin{array}{l}\text { Effect esti- } \\
\text { mate }^{a}\end{array}$ & P valueb & Study ID \\
\hline Long-term $\mathrm{PaO}_{2}(\mathrm{mmHg})$ & $\begin{array}{l}\text { Mean (SD): } 81.87 \\
(15.27)\end{array}$ & $\begin{array}{l}\text { Mean (SD): } 82.22 \\
(15.64)\end{array}$ & $\begin{array}{l}\mathrm{MD}-0.35,95 \% \\
\mathrm{Cl}-5.02 \text { to } 4.32\end{array}$ & & Cong 2019 \\
\hline Long-term $\mathrm{SpO}_{2}(\%)$ & $\begin{array}{l}\text { Mean (SD): } 87.83 \\
(8.16 \%)\end{array}$ & $\begin{array}{l}\text { Mean (SD): } 88.65 \\
(7.15)\end{array}$ & $\begin{array}{l}\mathrm{MD}-0.82,95 \% \\
\mathrm{Cl}-3.14 \text { to } 1.50\end{array}$ & & Cong 2019 \\
\hline Long-term $\mathrm{SpO}_{2}(\%)$ & $\begin{array}{l}\text { Mean (SD): } 91.93 \\
(4.35)\end{array}$ & $\begin{array}{l}\text { Mean (SD): } 92.75 \\
(4.07)\end{array}$ & $\begin{array}{l}\mathrm{MD}-0.82,95 \% \\
\mathrm{Cl}-2.09 \text { to } 0.45\end{array}$ & & Cong 2019 \\
\hline $\begin{array}{l}\text { Short-term } \mathrm{PaCO}_{\mathbf{2}}(\mathrm{mmHg}) \\
\text { (6 to } 12 \text { hours) }\end{array}$ & $\begin{array}{l}\text { Mean }(95 \% \mathrm{Cl}) 38.2 \\
(37.6 \text { to } 38.9)\end{array}$ & $\begin{array}{l}\text { Mean }(95 \% \mathrm{Cl}) 39.3 \\
(38.6 \text { to } 40.0)\end{array}$ & & 0.19 & Stephan 2015 \\
\hline Long-term $\mathrm{PaCO}_{2}(\mathrm{mmHg})$ & $\begin{array}{l}\text { Mean (SD) } 81.87 \\
(15.27)\end{array}$ & $\begin{array}{l}\text { Mean (SD) } 82.22 \\
(15.64)\end{array}$ & $\begin{array}{l}\text { MD }-0.35,95 \% \\
C l-5.02 \text { to } 4.32\end{array}$ & & Cong 2019 \\
\hline
\end{tabular}


Table 6. Comparison 2 (HFNC vs NIV or NIPPV): continuous outcomes for single studies (Continued)

\begin{tabular}{|c|c|c|c|c|c|}
\hline $\begin{array}{l}\text { Long-term respiratory rate } \\
\text { (breaths/min) }\end{array}$ & Mean (SD) 22.4 (4.4) & Mean (SD) 21 (4.5) & $\begin{array}{l}\text { MD } 1.40,95 \% \\
\mathrm{Cl}-1.36 \text { to } 4.16\end{array}$ & & Jing 2019 \\
\hline Length of hospital stay (days) & $\begin{array}{l}\text { Median (IQR): } 23 \text { (14 } \\
\text { to } 46)\end{array}$ & $\begin{array}{l}\text { Median (IQR): } 26 \text { (16 } \\
\text { to } 37 \text { ) }\end{array}$ & & Not reported & $\begin{array}{l}\text { Hernandez } \\
2016 a\end{array}$ \\
\hline Length of hospital stay (days) & $\begin{array}{l}\text { Median (IQR) } 13 \text { (9 to } \\
\text { 22) }\end{array}$ & $\begin{array}{l}\text { Median (IQR) } 14 \text { ( } 9 \text { to } \\
\text { 20) }\end{array}$ & & 0.59 & Stephan 2015 \\
\hline Length of hospital stay (days) & $\begin{array}{l}\text { Mean (SD): } 18.04 \\
(6.15)\end{array}$ & Mean (SD): 18.31 & $\begin{array}{l}\text { MD }-0.27 \text { days, } \\
95 \% \mathrm{Cl}-2.26 \\
\text { to } 1.72\end{array}$ & & Cong 2019 \\
\hline
\end{tabular}

acalculated using RevMan Web 2019

bas reported by study authors

$\mathrm{Cl}$ : confidence interval

ICU: intensive care unit

IQR: interquartile range

MD: mean difference

$\mathrm{PaCO}_{2}$ : partial pressure of carbon dioxide in arterial blood

$\mathrm{PaO}_{2} / \mathrm{FiO}_{2}$ : ratio of partial pressure of arterial oxygen to fraction of inspired oxygen

Table 7. Comparison 2 (HFNC vs NIV or NIPPV): summary effects of additional outcomes

\begin{tabular}{|c|c|c|c|c|}
\hline $\begin{array}{l}\text { Additional out- } \\
\text { comes }\end{array}$ & Study IDs & $\begin{array}{l}\text { Effect estimate } \\
\text { (short-term) }\end{array}$ & $\begin{array}{l}\text { Effect estimate } \\
\text { (long-term) }\end{array}$ & Comment \\
\hline $\begin{array}{l}\text { Duration of res- } \\
\text { piratory sup- } \\
\text { port }\end{array}$ & $\begin{array}{l}\text { Cong 2019; Jing } \\
2019\end{array}$ & $\begin{array}{l}\text { MD }-6.12 \text { hours, } 95 \% \mathrm{Cl}-54.61 \text { to } 42.37 ; 2 \\
\text { studies, } 210 \text { participants; } \mathrm{I}^{2}=60 \% \text {; Analysis } \\
2.7\end{array}$ & - & $\begin{array}{l}\text { We noted a wide } \\
\text { variation in re- } \\
\text { sults between } \\
\text { these studies; } \\
\text { this variation } \\
\text { may be caused } \\
\text { by differences in } \\
\text { illness severity in } \\
\text { the included par- } \\
\text { ticipants in each } \\
\text { study. }\end{array}$ \\
\hline
\end{tabular}

\begin{tabular}{lll}
\hline Long-term & Jing 2019; & MD $-31.67 \mathrm{mmHg}, 95 \% \mathrm{Cl}$ \\
$\mathbf{P a O}_{\mathbf{2}} / \mathbf{F i O}_{\mathbf{2}}$ & Stephan 2015 & -49.37 to $-13.97 ; 2$ stud- \\
& & ies, 344 participants; $\mathbf{1}^{2}=$ \\
& & $0 \%$; Analysis 2.8 \\
\hline
\end{tabular}

\begin{tabular}{|c|c|c|c|}
\hline $\mathrm{PaO}_{2}$ & $\begin{array}{l}\text { Cong 2019; Frat } \\
2015\end{array}$ & $\begin{array}{l}\text { MD }-9.57 \mathrm{mmHg}, 95 \% \mathrm{Cl}-30.25 \text { to } 11.11 ; 2 \\
\text { studies, } 384 \text { participants; } \mathrm{I}^{2}=89 \% \text {; Analysis } \\
2.9\end{array}$ & $\begin{array}{l}\mathrm{MD}-0.35 \mathrm{mmHg}, 95 \% \mathrm{Cl} \\
-5.02 \text { to } 4.32 ; 1 \text { study, } 168 \\
\text { participants; Table } 6\end{array}$ \\
\hline $\mathrm{SpO}_{2}$ & Cong 2019 & $\begin{array}{l}\text { MD }-0.82 \%, 95 \% \mathrm{Cl}-3.14 \text { to } 1.50 ; 1 \text { study, } \\
168 \text { participants; Table } 6\end{array}$ & $\begin{array}{l}\text { MD }-0.82 \%, 95 \% \mathrm{Cl}-2.09 \\
\text { to } 0.45 ; 1 \text { study, } 168 \text { par- } \\
\text { ticipants; Table } 6\end{array}$ \\
\hline $\mathrm{PaCO}_{2}$ & $\begin{array}{l}\text { Cong 2019; Frat } \\
\text { 2015; Jing 2019; } \\
\text { Stephan } 2015\end{array}$ & $\begin{array}{l}\text { MD }-0.46 \mathrm{mmHg}, 95 \% \mathrm{Cl}-2.08 \text { to } 1.16 ; 4 \\
\text { studies, } 1254 \text { participants; } \mathrm{I}^{2}=49 \% \text {; Analy- } \\
\text { sis } 2.10\end{array}$ & $\begin{array}{l}\mathrm{MD}-1.80 \mathrm{mmHg}, 95 \% \mathrm{Cl} \\
-5.57 \text { to } 1.98 ; 2 \text { studies, }\end{array}$ \\
\hline
\end{tabular}


Table 7. Comparison 2 (HFNC vs NIV or NIPPV): summary effects of additional outcomes (Continued)

208 participants; $12=0 \%$;

Analysis 2.10

\begin{tabular}{|c|c|c|c|c|}
\hline Respiratory rate & $\begin{array}{l}\text { Chanques 2013; } \\
\text { Frat 2015; Jing } \\
\text { 2019; Stephan } \\
2015\end{array}$ & $\begin{array}{l}\text { MD }-1.06 \text { breaths } / \mathrm{min}, 95 \% \mathrm{Cl}-1.80 \text { to } \\
-0.32 ; 4 \text { studies, } 1090 \text { participants; }\left.\right|^{2}=0 \% \text {; } \\
\text { Analysis } 2.11\end{array}$ & $\begin{array}{l}\text { MD } 1.40 \text { breaths/min, } \\
95 \% \mathrm{Cl}-1.36 \text { to } 4.16 ; 1 \\
\text { study, } 40 \text { participants; } \\
\text { Table } 6\end{array}$ & \\
\hline $\begin{array}{l}\text { Other adverse } \\
\text { events Ventila- } \\
\text { tor-acquired tra- } \\
\text { cheobronchitis }\end{array}$ & $\begin{array}{l}\text { Hernandez } \\
\text { 2016a }\end{array}$ & $\begin{array}{l}\text { RR } 0.66,95 \% \mathrm{Cl} 0.32 \text { to } 1.38 ; 1 \text { study, } 604 \\
\text { participants; Table } 5\end{array}$ & - & \\
\hline $\begin{array}{l}\text { Length of hospi- } \\
\text { tal stay }\end{array}$ & Cong 2019 & $\begin{array}{l}\text { MD }-0.27 \text { days, } 95 \% \mathrm{Cl}-2.26 \text { to } 1.72 ; 1 \text { study, } \\
168 \text { participants; Table } 6\end{array}$ & - & $\begin{array}{l}\text { Additional data } \\
\text { available from } \\
2 \text { studies (Her- } \\
\text { nandez 2016a; } \\
\text { Stephan 2015)ab; } \\
\text { see Table } 6\end{array}$ \\
\hline
\end{tabular}

\begin{tabular}{lll}
$\begin{array}{l}\text { Other partici- } \\
\text { pant-reported } \\
\text { outcomes }\end{array}$ & $\begin{array}{l}\text { Frat } 2015 ; \\
\text { Stephan } 2015\end{array}$ & $\begin{array}{l}\text { RR } 1.05,95 \% \mathrm{Cl} 0.74 \text { to } 1.48 ; 2 \text { studies, } 1023 \\
\text { participants; } 12=85 \% \text {; Analysis } 2.12\end{array}$ \\
Dyspnoea & & \\
\hline
\end{tabular}

aWe did not combine data from these studies in analyses because data were reported as median values

bFrom visual inspection, we noted that these data were likely to be right skewed due to the comparable magnitudes of the mean and standard deviation. This is expected for outcomes such as length of hospital stay due to most participants being discharged in a short time period with some outliers staying significantly longer. However, right skew introduces artefact into calculation of the effect estimate, limiting the interpretation of the result.

Cl: confidence interval

MD: mean difference

$\mathrm{PaCO}_{2}$ : partial pressure of carbon dioxide in arterial blood

$\mathrm{PaO}_{2} / \mathrm{FiO}_{2}$ : ratio of partial pressure of arterial oxygen to fraction of inspired oxygen

RR: risk ratio

$\mathrm{SpO}_{2}$ : oxygen saturation

Table 8. Comparison 2 (HFNC vs NIV or NIPPV): sensitivity analysis

Risk of selection: studies excluded from primary analysis owing to high or unclear risk of selection bias for random sequence generation or allocation concealment

\begin{tabular}{|c|c|c|}
\hline Important outcomes & Excluded studies & Effect of sensitivity analysis \\
\hline Failure of treatment & $\begin{array}{l}\text { Frat 2015; Shebl 2018; } \\
\text { Stephan } 2015\end{array}$ & Interpretation of the effect estimate remained the same \\
\hline In-hospital mortality & $\begin{array}{l}\text { Frat 2015; Shebl 2018; } \\
\text { Stephan } 2015\end{array}$ & Interpretation of the effect estimate remained the same \\
\hline $\begin{array}{l}\text { Important adverse events: } \\
\text { pneumonia, or barotrauma }\end{array}$ & NA & NA. Only one study included in primary analyses for these outcomes \\
\hline
\end{tabular}


Table 8. Comparison 2 (HFNC vs NIV or NIPPV): sensitivity analysis (Continued)

\begin{tabular}{lll} 
Length of ICU stay & Frat 2015 & $\begin{array}{l}\text { Interpretation of the effect estimate remained the same (only one } \\
\text { study remaining in analysis) }\end{array}$ \\
\hline $\mathrm{PaO}_{2} / \mathrm{FiO}_{2}$ up to 24 hours & Frat 2015; Stephan 2015 & $\begin{array}{l}\text { Effect estimate indicated no evidence of a difference between types } \\
\text { of respiratory support used (MD }-9.30 \mathrm{mmHg}, 95 \% \mathrm{Cl}-80.37 \text { to } 61.77 ; \\
1 \text { study, 40 participants) }\end{array}$ \\
\hline Comfort (short-term) & Frat 2015 & $\begin{array}{l}\text { Interpretation of the effect estimate remained the same (only one } \\
\text { study remaining in analysis) }\end{array}$ \\
\hline Comfort (long-term) & NA & NA. Only one study included in primary analysis for this outcome
\end{tabular}

\section{High risks of other bias: studies excluded from primary analysis owing to high risks of other bias}

\begin{tabular}{|c|c|c|}
\hline Outcome & Excluded studies & Effect of sensitivity analysis \\
\hline Failure of treatment & $\begin{array}{l}\text { Frat } 2015 \text { (selective reporting } \\
\text { bias, and differences in treat- } \\
\text { ment in the HFNC group) }\end{array}$ & Interpretation of the effect estimate remained the same \\
\hline In-hospital mortality & $\begin{array}{l}\text { Frat } 2015 \text { (selective reporting } \\
\text { bias, and differences in treat- } \\
\text { ment in the HFNC group) }\end{array}$ & Interpretation of the effect estimate remained the same \\
\hline $\begin{array}{l}\text { Important adverse events: } \\
\text { pneumonia }\end{array}$ & $\begin{array}{l}\text { Frat } 2015 \text { (selective reporting } \\
\text { bias, and differences in treat- } \\
\text { ment in the HFNC group) }\end{array}$ & Interpretation of the effect estimate remained the same \\
\hline $\begin{array}{l}\text { Important adverse events: } \\
\text { barotrauma }\end{array}$ & NA & NA. Only one study included in primary analysis for this outcome \\
\hline Length of ICU stay & $\begin{array}{l}\text { Frat } 2015 \text { (selective reporting } \\
\text { bias, and differences in treat- } \\
\text { ment in the HFNC group) }\end{array}$ & $\begin{array}{l}\text { Interpretation of the effect estimate remained the same (only one } \\
\text { study remaining in analysis) }\end{array}$ \\
\hline $\mathrm{PaO}_{2} / \mathrm{FiO}_{2}$ up to 24 hours & $\begin{array}{l}\text { Frat } 2015 \text { (selective reporting } \\
\text { bias, and differences in treat- } \\
\text { ment in the HFNC group) }\end{array}$ & Interpretation of the effect estimate remained the same \\
\hline Comfort (short-term) & $\begin{array}{l}\text { Frat } 2015 \text { (selective reporting } \\
\text { bias, and differences in treat- } \\
\text { ment in the HFNC group) }\end{array}$ & $\begin{array}{l}\text { Interpretation of the effect estimate remained the same (only one } \\
\text { study remaining in analysis) }\end{array}$ \\
\hline Comfort (long-term) & NA & NA. Only one study included in primary analysis for this outcome \\
\hline
\end{tabular}

\section{Fixed effect versus random effects: we re-analysed the data using a fixed-effect model}

\begin{tabular}{ll}
\hline Outcomes & Effect of sensitivity analysis \\
\hline Failure of treatment & Interpretation of the effect estimate for all outcomes remained the same \\
In-hospital mortality & \\
Important adverse events: \\
pneumonia or barotrauma \\
Length of ICU stay \\
$\mathrm{PaO}_{2} / \mathrm{FiO}_{2}$ up to 24 hours
\end{tabular}


Table 8. Comparison 2 (HFNC vs NIV or NIPPV): sensitivity analysis (Continued)

Comfort (short-term)

Comfort (long-term)

\section{Funding: studies excluded from analysis in which funding was from commercial sources}

\begin{tabular}{lll}
\hline Outcome & Excluded studies & Effect of sensitivity analysis \\
\hline Failure of treatment & Frat 2015 & Interpretation of the effect estimate remained the same \\
\hline In-hospital mortality & Frat 2015 & Interpretation of the effect estimate remained the same \\
\hline $\begin{array}{l}\text { Important adverse events: } \\
\text { pneumonia }\end{array}$ & Frat 2015 & Interpretation of the effect estimate remained the same \\
\hline $\begin{array}{l}\text { Important adverse events: } \\
\text { barotrauma }\end{array}$ & Frat 2015 & NA. Only one study included in primary analysis for this outcome \\
\hline $\begin{array}{l}\text { Length of ICU stay } \\
\text { PaO } 2 \text { /FiO } 2 \text { up to } 24 \text { hours }\end{array}$ & Frat 2015 2015 & $\begin{array}{l}\text { Interpretation of the effect estimate remained the same (only one } \\
\text { study remaining in analysis) }\end{array}$ \\
\hline $\begin{array}{l}\text { Comfort (short-term) } \\
\text { Comfort (long-term) }\end{array}$ & Frat 2015 & Interpretation of the effect estimate remained the same \\
\hline
\end{tabular}

$\mathrm{Cl}$ : confidence interval

ICU: intensive care unit

MD: mean difference

NA: not applicable

$\mathrm{PaO}_{2} / \mathrm{FiO}_{2}$ : partial pressure of arterial oxygen/fraction of inspired oxygen

\section{A P PEN DICES}

\section{Appendix 1. CENTRAL search strategy}

\#1 (((high flow or highflow or nasal $\left.{ }^{\star}\right)$ near can?ul $\left.{ }^{\star}\right)$ or HFNC or (nasal near (high flow highflow or prong)) or Vapotherm or Optiflow)

\section{Appendix 2. MEDLINE search strategy}

1 exp Oxygen/

2 exp Oxygen Inhalation Therapy/

3 Cannula/

4 (1 or 2$)$ and 3

5 ((high flow or highflow or high frequency or nasal $\left.{ }^{\star}\right)$ adj6 can? $\left.^{\star} l^{\star}\right)$.af.

6 ((high flow or highflow or high frequency or prong*) adj6 nasal $\left.{ }^{\star}\right)$.af.

7 ((high flow or highflow or high frequency) adj4 oxygen $\left.{ }^{\star}\right)$.af.

8 (HFNC or HFNP or Vapotherm or Optiflow).af. 
9 transnasal insufflation.af.

104 or 5 or 6 or 7 or 8 or 9

11 ((randomized controlled trial or controlled clinical trial).pt. or randomi?ed.ab. or placebo.ab. or drug therapy.fs. or randomly.ab. or trial.ab. or groups.ab.) not (exp animals/ not humans.sh.)

1210 and 11

\section{Appendix 3. Embase search strategy}

1 exp nasal cannula/

2 ((high flow or highflow or high frequency or nasal $\left.{ }^{\star}\right)$ adj6 can?ul*).af.

3 ((high flow or highflow or high frequency or prong*) adj6 nasal).af.

4 ((high flow or highflow or high frequency) adj4 oxygen $\left.{ }^{\star}\right)$.af.

5 (HFNC or HNFP or Vapotherm or Optiflow).af.

6 transnasal insufflation.af.

71 or 2 or 3 or 4 or 5 or 6

8 (randomized controlled trial/ or controlled clinical study/ or random\$.ti,ab. or randomization/ or intermethod comparison/ or placebo.ti,ab. or (compare or compared or comparison).ti. or ((evaluated or evaluate or evaluating or assessed or assess) and (compare or compared or comparing or comparison)).ab. or (open adj label).ti,ab. or ((double or single or doubly or singly) adj (blind or blinded or blindly)).ti,ab. or double blind procedure/ or parallel group\$1.ti,ab. or (crossover or cross over).ti,ab. or ((assign\$ or match or matched or allocation) adj5 (alternate or group $\$ 1$ or intervention $\$ 1$ or patient $\$ 1$ or subject $\$ 1$ or participant $\$ 1)$ ).ti,ab. or (assigned or allocated).ti,ab. or (controlled adj7 (study or design or trial)).ti,ab. or (volunteer or volunteers).ti,ab. or human experiment/ or trial.ti.) not (((random\$ adj sampl\$adj7 (cross section $\$$ or questionnaire $\$ 1$ or survey $\$$ or database $\$ 1)$ ).ti,ab. not (comparative study/ or controlled study/ or randomi? ed controlled.ti,ab. or randomly assigned.ti,ab.)) or (cross-sectional study/ not (randomized controlled trial/ or controlled clinical study/ or controlled study/ or randomi?ed controlled.ti,ab. or control group\$1.ti,ab.)) or (((case adj control\$) and random\$) not randomi?ed controlled).ti,ab. or (nonrandom\$ not random\$).ti,ab. or Random field\$.ti,ab. or (random cluster adj3 sampl\$).ti,ab. or ((rat or rats or mouse or mice or swine or porcine or murine or sheep or lambs or pigs or piglets or rabbit or rabbits or cat or cats or dog or dogs or cattle or bovine or monkey or monkeys or trout or marmoset\$1).ti. and animal experiment/) or (Animal experiment/ not (human experiment/ or human/)))

97 and 8

\section{Appendix 4. CINAHL search strategy}

S1 (((high flow or highflow or nasal*) N3 can?ul*) or HFNC or (nasal N3 (high flow or highflow or prong)) or Vapotherm or Optiflow) S2 ( (random* or (trial* N3 (controlled or clinical)) or placebo* or prospective or multicenter) or ((blind* or mask*) N3 (single or double or triple or treble)))

S3 S1 and S2

\section{Appendix 5. ISI Web of Science search strategy}

\#1 TS=(((high flow or highflow or nasal $\left.{ }^{\star}\right)$ SAME can?ul*) or HFNC or (nasal SAME (high flow or highflow or prong)) or Vapotherm or Optiflow)

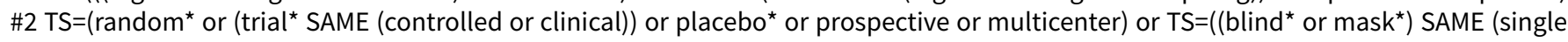
or double or triple or treble))

\#3 \#1 and \#2

\section{Appendix 6. COVID-19 Register search strategy}

(nasal* or cannul $^{\star}$ or oxygen or prong*) and (high flow or high frequency)

\section{Appendix 7. Data extraction template}

\section{Completed by}

Date 
(Continued)

Study ID

\begin{tabular}{|c|c|}
\hline Methods & $\begin{array}{l}\text { Study design: } \\
\text { Multicentre or single-centre: } \\
\text { Country: } \\
\text { Study aim: }\end{array}$ \\
\hline Participants & 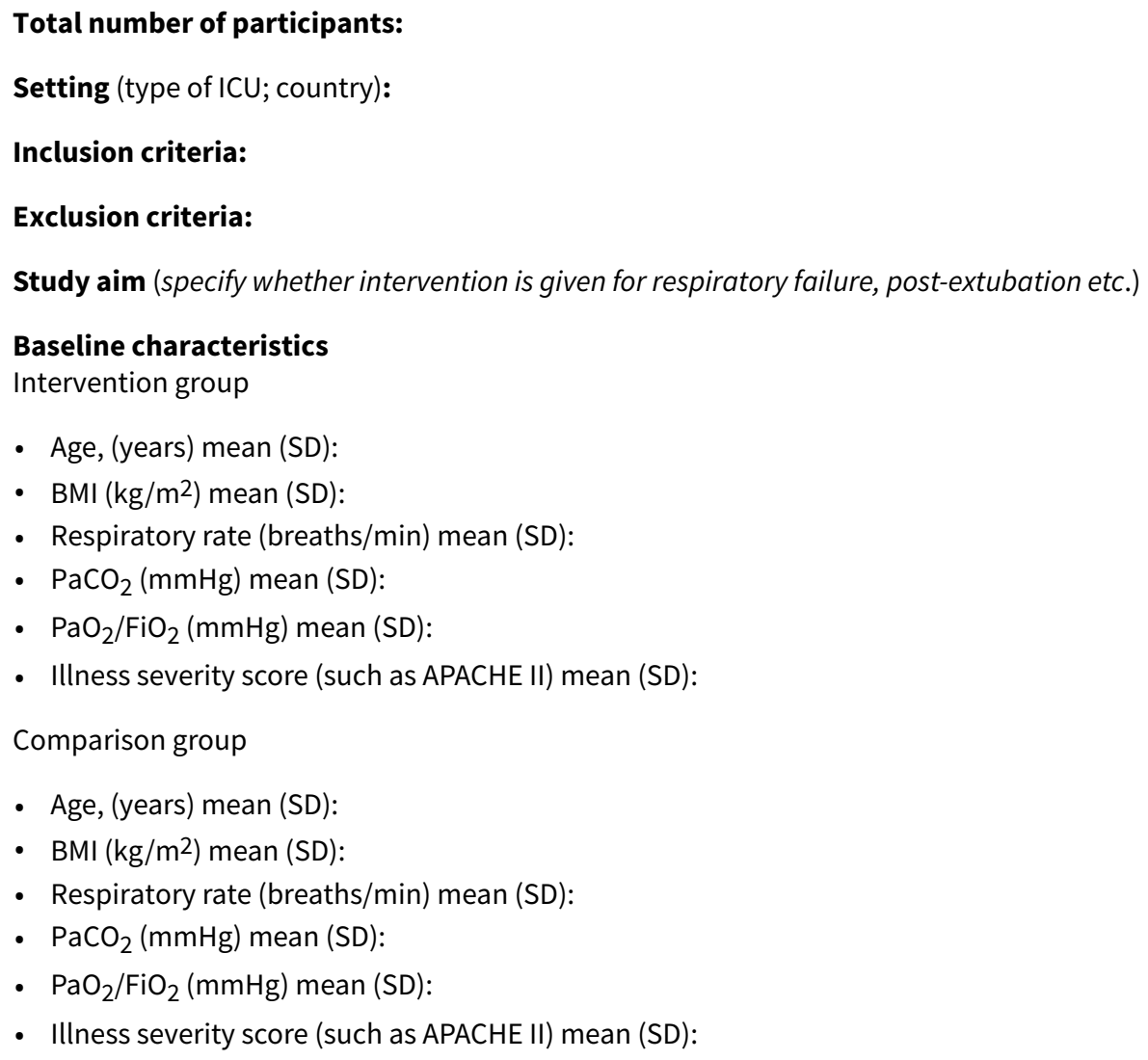 \\
\hline
\end{tabular}

Interventions

Intervention group

- Randomized, $\mathrm{n}=$; losses (with reasons) = ; analysed, $\mathrm{n}=$

- Details (include type of device; size of nasal cannulae; flow rate; duration; washout period in crossover studies):

Comparison group

- Randomized, $\mathrm{n}=$; losses (with reasons) = ; analysed, $\mathrm{n}=$

- Details (include type of device; flow rate; duration; washout period in cross-over studies):

Outcomes

Outcomes measured/reported by study authors:

Outcomes relevant to the review :

\begin{tabular}{ll}
\hline Outcome data & If study is a cross-over design, are the data reported separately for the first cross-over period \\
\hline Notes & Funding/declarations of interest: \\
& Study dates: \\
& Additional notes: \\
\hline
\end{tabular}




\title{
Outcome data - dichotomous variables
}

\section{Name of outcome:}

Time point of measurement:

\section{Intervention group}

Number of events

Total number of participants in the group

Control group

\section{Outcome data - continuous data}

\section{Name of outcome:}

Intervention group

Mean SD Total number of participants in the group

Control group

Mean SD Total number of participants in the group

\section{Risk of bias table for randomised controlled trials}

\begin{tabular}{ll}
\hline Domain & High/Low/ Judgement \\
& Unclear \\
\hline
\end{tabular}

\section{Random sequence generation (selection bias)}

\author{
Allocation concealment \\ (selection bias) \\ Blinding of participants and personnel (performance bias)
}


(Continued)

Blinding of outcome assessors (detection bias)

Incomplete outcome data (attrition bias)

Selective reporting

(reporting bias)

Other bias

WHAT'S NEW

\begin{tabular}{lll}
\hline Date & Event & Description \\
\hline 24 February 2021 & Amended & $\begin{array}{l}\text { Minor superscript changes made to Footnotes in Additional ta- } \\
\text { bles. Author affiliation correction and } 12 \text { change from } 97 \% \text { to } 96 \% \\
\text { in Comparison 1: HFNC versus standard oxygen therapy, Impor- } \\
\text { tant outcomes/Comfort. }\end{array}$ \\
& & \\
&
\end{tabular}

\section{H I S T O R Y}

Protocol first published: Issue 11, 2012

Review first published: Issue 5, 2017

\begin{tabular}{lll}
\hline Date & Event & Description \\
\hline 16 June 2020 & $\begin{array}{l}\text { New citation required and conclusions } \\
\text { have changed }\end{array}$ & $\begin{array}{l}\text { The review findings were changed as follows with this update: } \\
\end{array}$ \\
& $\begin{array}{l}\text { We found that treatment failure may be reduced with HFNC } \\
\text { compared to standard oxygen but there remained no evidence } \\
\text { of a difference when HFNC was compared to NIV/NIPPV } \\
\text { - We increased the certainty of the evidence for mortality to } \\
\text { moderate when HFNC was compared to standard oxygen ther- } \\
\text { apy }\end{array}$
\end{tabular}

We continued to find low or very low-certainty evidence for all other important outcomes across both comparisons. Additional evidence is still required to increase certainty in the findings

$\begin{array}{ll}\text { New search has been performed } & \text { We updated the review and made the following amendments. } \\ & \text { - We added one new author to the review (PB) } \\ \text { - We re-ran the searches using new updated search strategies } & \text { - We found } 20 \text { new studies and incorporated data from these } \\ \text { studies into the review. In addition, we found } 19 \text { studies await- } & \text { ing classification and } 51 \text { ongoing studies } \\ \text { - We separated the findings into two comparison groups (stan- } & \text { dard oxygen therapy, and NIV or NIPPV). We included a 'Sum- } \\ & \text { mary of findings' table for each of these comparisons }\end{array}$




\section{CONTRIBUTIONS OF AUTHORS}

Contributions made by authors in the previous version of the review can be found in Corley 2017.

Sharon R Lewis (SRL), Philip E Baker (PEB), Roses Parker (RP), Andrew F Smith (AFS).

Co-ordinating the review update, undertaking manual searches, organizing retrieval of papers, screening retrieved papers against inclusion criteria, extracting data from papers, conducting 'Risk of bias' assessments, entering data in RevMan Web 2019, analysing and interpreting data: PEB and SRL

Writing the review: PEB, RP and SRL

Contacting study authors for additional information: PEB

Taking responsibility for reading and checking the review before submission: PEB, SRL, RP, AFS.

\section{DECLARATIONSOF INTEREST}

Sharon R Lewis (SRL), Philip E Baker (PEB), Roses Parker (RP), Andrew F Smith (AFS).

Review authors SRL, PEB, RP and AFS have no conflicts of interest to declare.

\section{DIFFERENCES BETWEEN PROTOCOLAND REVIEW}

Changes between protocol and review made in the previous publication are reported in Corley 2017.

\section{Differences between the current and previous version of the review}

We made the following changes to Corley 2017.

- Authors: we added two new authors to the review team (Philip Baker and Roses Parker) and we removed six authors from the review team who were no longer able to contribute to the review owing to time constraints: Amanda Corley, Claire M Rickard, Leanne M Aitken, Amy Johnston, Adrian Barnett, John F Fraser.

- Objectives: we re-worded the review objectives, using a single sentence, following current Cochrane guidance.

- Types of studies: we excluded cluster- and quasi-randomized studies. Quasi-randomized studies were originally included as it was expected that limited data would be available for this review. However, as sufficient high-quality studies are now published for this topic, we believed that it was appropriate to exclude both these study designs from this update.

- Types of interventions: we made a greater distinction between the different types of respiratory support in the review. We separated the interventions into two comparison groups (standard oxygen therapy; and NIV or NIPPV). We made edits to other sections of the text in order to specify the separate management of these two comparison groups. For consistency, we used the term 'standard oxygen therapy' throughout the review to refer to low-flow oxygen, conventional oxygen therapy and standard oxygen therapy; we had previously used these terms interchangeably throughout the review. We clarified that we included standard oxygen therapy with or without humidification and heating.

- Types of outcomes: we removed the term 'primary outcome', instead splitting the outcomes into 'important' and 'additional' outcomes, where the 'important outcomes' appear in the 'Summary of findings' tables. For the outcome 'treatment failure', we clarified that this was escalation of respiratory support that included NIV, as well as NIPPV or invasive ventilation depending on the initial respiratory therapy. For the outcome 'adverse events', we specified and collected data only on specific outcomes. We found that study authors varied in whether or not they defined outcomes as 'adverse events'. We, therefore, collected data on: respiratory infections (pneumonia, and tracheobronchitis), abdominal distension, and nasal mucosa or skin trauma. Data for other adverse events that were previously reported can be found in Corley 2017. We also clarified in this section, that we separated data for respiratory effects and participantreported outcomes according to short-term and longer-term effects. We removed $\mathrm{PCO}_{2}$ as an outcome; this is a predictor of $\mathrm{PaCO}_{2}$ which is commonly reported in studies.

- Search methods: we updated the search strategies following the advice and support of the Information Specialist for the Cochrane Emergency and Critical Care Group. In this review update, we did not separately search for abstracts from conference proceedings (for the European Society of Intensive Care Medicine, the Australia and New Zealand Intensive Care Society, the Society of Critical Care Medicine, and the American Thoracic Society); we expected that publications from these conference proceedings would be included in the comprehensive database search strategies.

- Data extraction and management: we edited the data extraction forms, in order to use a template that was more consistent with the tables in Characteristics of included studies. In addition, we added detail about the information collected during data extraction.

- Measures of treatment effect: when dealing with continuous data presented on different scales, we added that we would aim to scale and invert the outcome data to allow calculation of a mean difference before calculating standardized mean difference. 
- Assessment of risk of bias: we reduced the text in this section. Rather than making 'Risk of bias' judgements on all studies, we only made judgements on studies for which we reported outcome data; we specified this in this Methods section. We made judgements separately for detection bias according to whether outcomes were subjective (participant-reported) or objective (all other outcomes).

- Sensitivity analysis: we provided additional clarity to the sensitivity analyses. We added sensitivity analysis to explore the effects of high risk of bias in domains other than selection bias (as we performed a separate sensitivity analysis for this domain). In addition, we performed further sensitivity analyses to exclude studies that were commercially funded with a potential conflict of interest on advice from the Cochrane Emergency and Critical Care Group editorial team. We also used sensitivity analysis on all of the outcomes in the 'Summary of findings' tables, because we used this information when assessing the certainty of the evidence with GRADE.

- Summary of findings: we conducted GRADE assessments for the two comparison groups introduced in this latest update.

\section{NOTES}

We would like to thank Harald Herkner (Content Editor); Cathal Walsh (Statistical Editor); and Georgine Imberger, Jean-Damien Ricard, and Kevin Dysart (Peer Reviewers) for help and editorial advice provided during preparation of the protocol (Corley 2012) for this systematic review.

\section{INDEX TERMS}

\section{Medical Subject Headings (MeSH)}

Critical Care [*methods]; Hospital Mortality; Intubation [adverse effects] [ ${ }^{*}$ methods]; Length of Stay; Oxygen Inhalation Therapy [adverse effects] [ ${ }^{\star}$ methods]; Patient Reported Outcome Measures; Randomized Controlled Trials as Topic; Respiratory Distress Syndrome [*therapy]; Treatment Failure

\section{MeSH check words}

Adult; Humans 